\title{
Microarray analysis of oxidative phosphorylation disorders
}

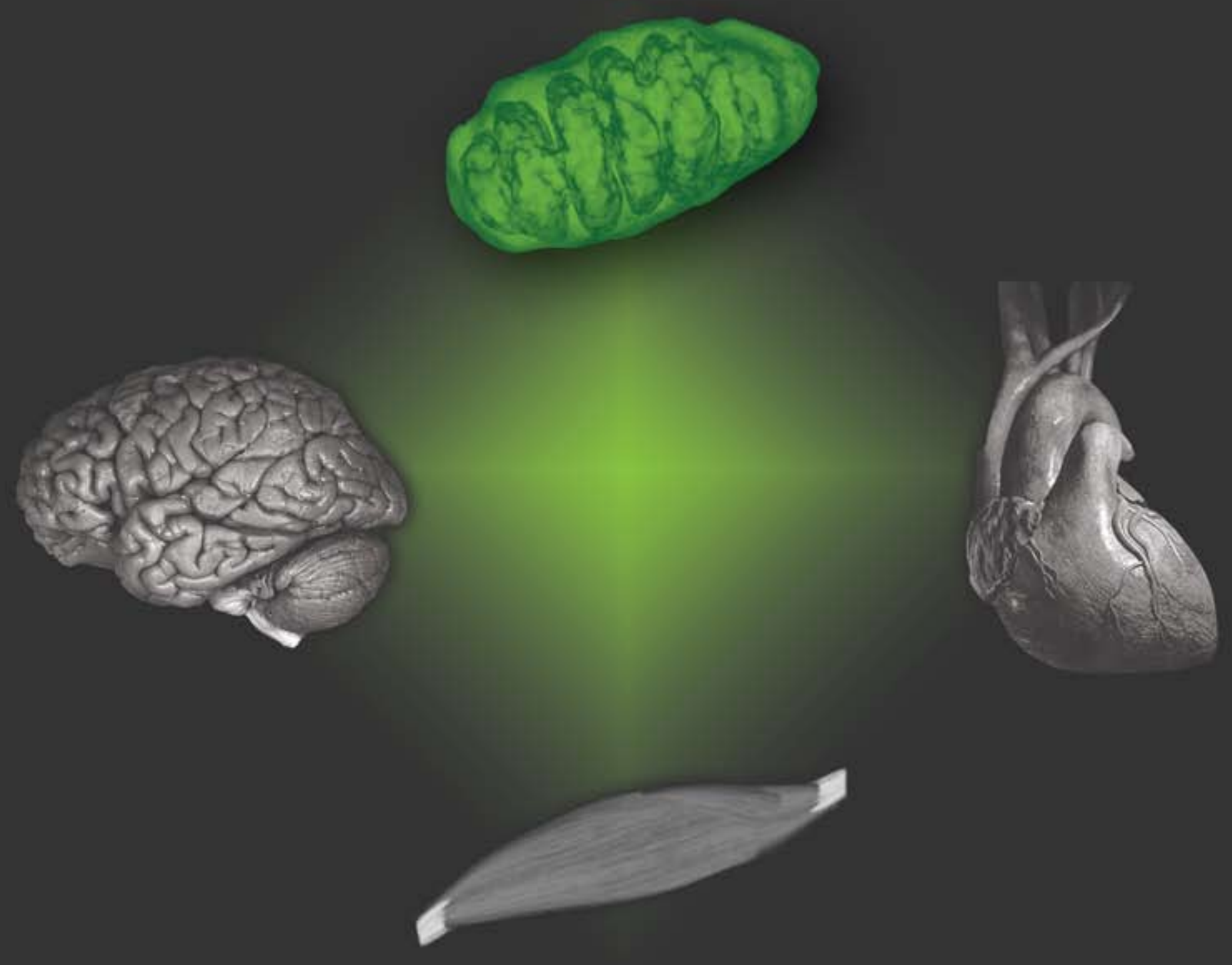

Rudy G.E. van Eijsden 
Microarray analysis of oxidative phosphorylation disorders 
(c) Rudy G.E. van Eijsden, Sittard, 2008

ISBN 978-90-8891-0449

\section{Cover design}

Rudy van Eijsden

Figure 1 of chapter 1

Lars Eijssen

Printed by

Proefschriftmaken.nl 


\title{
Microarray analysis of oxidative phosphorylation disorders
}

\author{
Proefschrift
}

ter verkrijging van de graad van doctor

aan de Universiteit Maastricht,

op gezag van de Rector Magnificus,

Pof. mr. G.P.M.F. Mols,

Volgens het besluit van het College van Decanen,

In het openbaar te verdedigen

op donderdag 22 mei 2008 om 12.00 uur

door

Rudy Gerardus Elisabeth van Eijsden

Geboren te Maastricht op 3 augustus 1977 


\section{Promotor}

Prof. dr. J.P.M. Geraedts

\section{Copromotor}

Dr. H.J.M. Smeets

\section{Beoordelingscommissie}

Prof. dr. B. G. Wouters (voorzitter)

Prof. dr. P. Van Hummelen (VIB, K.U. Leuven)

Prof. dr. A.K. Raap (LUMC, Leiden)

Dr. M. E. Rubio-Gozalbo (AzM, Maastricht)

Prof. dr. G. J. van der Vusse

The studies described in this thesis were supported by a grant of the Princess Beatrix Foundation (MAR99-0111) and performed at the Department of Genetics and Cell Biology, Research Institute Growth and Development (GROW), Maastricht University, The Netherlands. 
The beginning of knowledge is the discovery of something we do not understand - Frank Herbert - 



\section{CHAPTER 1}

General Introduction

\section{CHAPTER 2}

Chip-based mtDNA mutation screening enables fast and reliable genetic diagnosis of OXPHOS patients

\section{CHAPTER 3}

Gene expression profiling reveals complement mediated regeneration in skeletal muscle from Leigh syndrome patients with a SURF1 mutation

\section{CHAPTER 4}

Termination of damaged protein repair defines the occurrence of symptoms in carriers of the m.3243A $>\mathrm{G}$ tRNA ${ }^{\text {Leu }}$ mutation

\section{ChAPTER 5}

Increasing levels of the m.9176 $>\mathrm{C}$ mutation in monoclonal human skin fibroblasts induces changes in apoptosis and cytoskeleton organisation

\section{CHAPTER 6}

General Discussion

REFERENCES

SUMMARY

SamenVatting

DANKWOORD 



\section{CHAPTER 1}

General Introduction 
Mitochondrial genetics of oxidative phosphorylation disorders

Mitochondria are cellular organelles which are present in almost all eukaryotic cells. One of the most important functions of mitochondria is the production of energy in the form of ATP through the process of oxidative phosphorylation (OXPHOS). OXPHOS starts with a series of oxidationreduction reactions carried out by a group of enzymes embedded in the mitochondrial inner membrane referred to as the respiratory chain (figure 1 ). The respiratory chain consists of four enzyme complexes, OXPHOS complexes I to IV. During this process a reduction of $\mathrm{O}_{2}$ (respiration) and oxidation of $\mathrm{NADH}$ (and FADH2) takes place and an electrochemical gradient is created by the pumping of protons from the mitochondrial matrix to the mitochondrial inter-membrane space. The electrochemical proton gradient is the driving-force for the generation of ATP when protons flow back across the membrane. ATP is generated by the ATP synthase or ATPase complex (complex $\mathrm{V}$ ), which is the final OXPHOS complex. During this process of aerobic energy metabolism, reactive oxygen species (ROS) are formed as a by-product, making mitochondria the main intracellular source of ROS production but also an immediate target of ROS-induced damage.

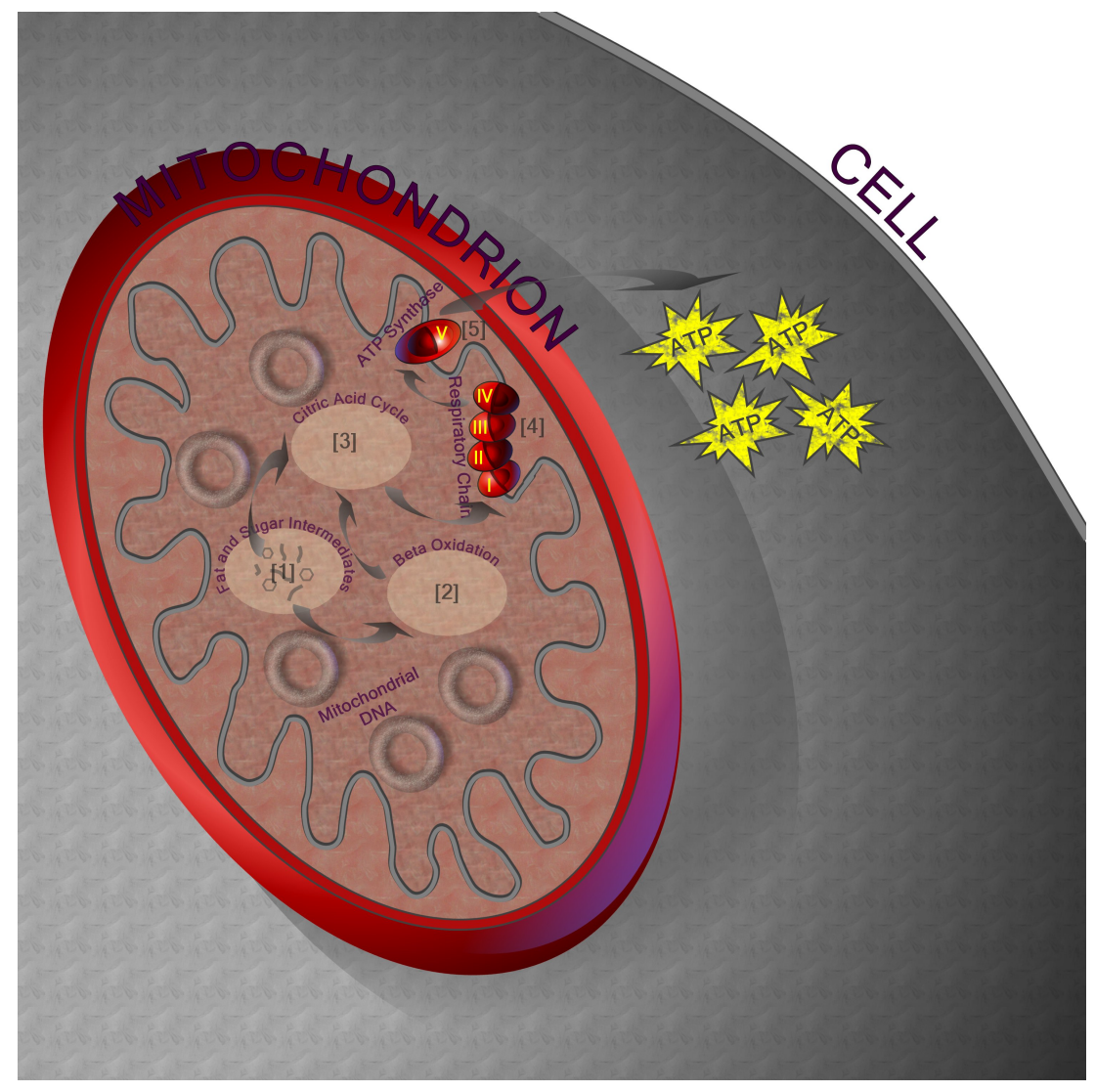

Figure 1: Mitochondria and the respiratory chain inside a cell

Fat and sugar metabolism (1) and beta oxidation (2) form intermediates for the citric acid cycle (3). Oxidation products $\mathrm{NADH}$ and $\mathrm{FADH}_{2}$ produced by the citric acid cycle enter into the respiratory chain (4) where an electrochemical proton gradient is created which drives the ATP synthase complex (5) to produce energy in the form of ATP. The numbers I to $\mathrm{V}$ represent the five OXPHOS complexes. 
Mitochondria are one of the few cell organelles which carry their own functional genome. The human mitochondrial DNA (mtDNA) molecule contains 16,569 bp and comprises 13 proteinencoding genes, 22 tRNA genes and 2 rRNA genes (figure 2) [1], and is inherited maternally. A cell is polyploid for the mtDNA, because each cell can contain hundreds to thousands of mitochondria and each mitochondrion contains 5 to 10 copies of the mtDNA. The proteins encoded by the mtDNA are all subunits of the different OXPHOS complexes. The mtDNA encodes seven subunits of complex I (NADH dehydrogenase), one subunit of complex III (b-c1 complex), three subunits of complex IV (cytochrome $c$ oxidase), and two subunits of complex V (ATPase). The other subunits of these complexes and the entire complex II are coded for by the nuclear DNA (nDNA). The mitochondrial tRNA and rRNA molecules are necessary for the translation and protein synthesis from these mitochondrial transcripts, because the mtDNA code differs from the nDNA code, e.g. UGA codes for tryptophan rather than being a stop codon as in the genetic code of the nDNA [2]. Pathogenic point mutations in one of the mtDNA protein encoding, tRNA or rRNA genes can lead to malfunctioning of OXPHOS and clinical symptoms. Additionally, mtDNA rearrangements (deletions/duplications) or depletions can have a similar effect. Copies of the mtDNA with pathogenic mutations usually coexist with wild-type mtDNA in the same cell or tissue, a situation referred to as heteroplasmy. Disease manifestations only occur when the level of mutated mtDNA molecules exceeds a cell or tissue specific threshold. Disorders in which OXPHOS deficiency is a central characteristic are referred to as oxidative phosphorylation disorders, or in short, OXPHOS disorders. MtDNA mutations can arise de novo, they can be acquired somatically, mainly due to ROS damage, or they can be maternally inherited and segregate in families. An inefficient OXPHOS function will often result in increased ROS production, thereby increasing the somatic mutation rate and causing protein and DNA damage. Besides mutations in these mtDNA genes, mutations in nuclear genes with a direct or indirect relation to OXPHOS may also result in OXPHOS deficiency. These genes may encode structural OXPHOS subunits, but it may also be genes involved in mtDNA replication and mitochondrial biogenesis, regulatory genes in the OXPHOS complex formation.

A hallmark of OXPHOS disorders is genetic and clinical heterogeneity. Genetic heterogeneity means that different mutations, i.e. mutations in different genes (mtDNA or nDNA) or at different sites in the same gene, can lead to the same clinical symptoms. Clinical heterogeneity refers to situations where the same mutation results in different clinical symptoms or syndromes. At least for mtDNA mutations, part of this can be explained by heteroplasmy and different mutation levels. Syndromic OXPHOS disorders which are caused by heteroplasmic mtDNA mutations and which have been studied for this thesis are Leigh syndrome (OMIM \#256000), Neuropathy, Ataxia, and Retinitis Pigmentosa (NARP, OMIM \#551500), and mitochondrial Myopathy, Encephalopathy, Lactic Acidosis, and Stroke-like episodes (OMIM \#540000). Leigh syndrome is genetically heterogeneous and can be caused by a variety of mutations in mtDNA encoded as well as in nDNA encoded genes. Leigh syndrome can for example be caused by the m.9176T>C mutation in the mtDNA encoded ATP6 gene [3-5], but also by mutations in the nuclear SURF1 gene 
which encodes a complex IV assembly factor [6-8]. A more common cause of Leigh syndrome and the milder NARP syndrome is a point mutation at position 8993 in the ATP6 gene, where a thymidine is mutated to a cytosine or a guanine $(\mathrm{m} .8993 \mathrm{~T}>\mathrm{C} / \mathrm{G})$. For this mutation, heteroplasmy is an important factor for clinical expression as the NARP symptoms usually manifest at mutation loads above $60 \%$, and severe Leigh syndrome above 90\% [9-11]. The clinical heterogeneity for this mutation can largely be explained by the heteroplasmy level, but this is not always the case. The m.3243A $>C$ mutation is an example of a mutation that leads to extreme clinical heterogeneity, which can not be explained by heteroplasmy differences only. This mutation may result in MELAS, cardiomyopathy, diabetes mellitus, deafness, renal failure, or a combination of these symptoms [12-21].

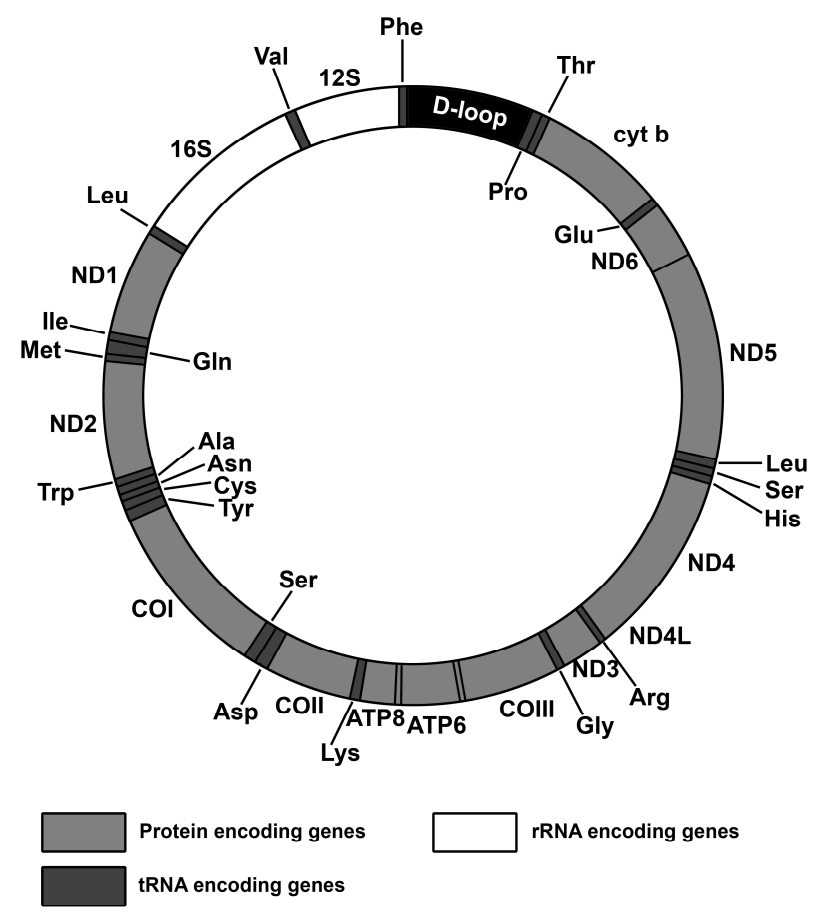

Figure 2: Schematic representation of the human mtDNA molecule

The image was downloaded and adapted from the MitoMap website: www.mitomap.org [22].

Diagnosis of OXPHOS disorders

A genetic diagnosis of OXHOS disorders can be difficult to achieve due to the genetic and clinical heterogeneity. Diagnosis of OXPHOS disease is mostly symptom based, supported by measurements of blood lactate and pyruvate levels, the activity of the separate OXPHOS complexes in muscle or cultured fibroblasts, and/or microscopy. A genetic diagnosis is required to confirm the clinical diagnosis, characterise the cause of the disease and predict the segregation pattern, and to provide more accurate information on prognosis and possible treatments. In a number of OXPHOS disorders, where symptoms are directly related to a particular mutation, a rapid genetic diagnosis is possible. However, often this can not be achieved due to the genetic 
and clinical heterogeneity of OXPHOS disorders, and the genetic defect may reside in the mtDNA or in one of the many candidate genes in the nDNA, providing a major challenge for genetic testing.

As the number of patients with the same mutation or a mutation in the same gene is fairly small, diagnostic centres have access to only relatively small numbers of patients in each disease category, making it difficult for each centre to develop consensus guidelines for the investigation of OXPHOS disorders. There are only limited clinical or laboratory guidelines for the investigation of suspected mitochondrial disease. The current workflow for a genetic diagnosis usually starts with screening for several mtDNA mutations which are most common in OXPHOS disorders and screening for deletions and depletions of the mtDNA. If symptoms are likely to be linked to a specific gene or specific genes, a candidate gene approach can be chosen where genes which previously have been related to the clinical symptoms are screened for mutations. When a diagnosis OXPHOS disease is likely, it makes sense to screen the entire mtDNA for mutations. In paediatric cases of OXPHOS disease, about $25 \%$ is caused by an mtDNA defect [23]. When mtDNA screening still does not lead to a genetic diagnosis and the suspicion of an OXPHOS disorder still holds, a more detailed investigation will be necessary, which will be more time consuming, costly, and with an uncertain success rate. Of course this has to be evaluated case by case, and depends on the guidance towards candidate genes based on symptoms, laboratory investigations and family data. When the size of the family and the number of patients is sufficient, linkage analysis can be carried out in order to identify a chromosomal region and characterise the genetic defect in candidate genes. Genetic linkage analysis in a consanguineous family with an isolated cytochrome $c$ oxidase ( $\mathrm{COX}$ ) deficiency indentified a locus containing the two candidate genes SCO1 and COX10, which are both involved in COX assembly, and COX10 appeared to contain a pathogenic mutation [24]. An alternative approach is a complementation study, where whole chromosomes or parts of chromosomes are transferred to cultured patient cells in order to try and compensate the OXPHOS defect. A prerequisite is that the OXPHOS defect can be measured in cell lines, which is not always the case. This approach will narrow down or will result in a list of candidate genes which subsequently can be screened for mutations. This strategy was used for the identification of chromosome 9 being the chromosome containing the genetic defect in Leigh syndrome with cytochrome c oxidase (COX) deficiency. Subsequently, linkage analysis was carried out to narrow down the region on chromosome 9 to a 7-cM interval on chromosome 9934, significantly decreasing the number of candidate genes for mutation screening. Finally, the SURF1 gene contained pathogenic mutations in these families with Leigh syndrome with COX deficiency [7].

The improvement and development of new and existing mutation detection technologies and their rapidly increasing capacity will provide an alternative for those cases in which clinical symptoms can not be directly linked to a specific gene, candidate gene list, or mtDNA mutation or when linkage analysis or complementation assays are not possible. As this is the majority of cases 
and mutation screening technology is evolving extremely rapidly, this might be the better alternative. High throughput gene expression analysis would also be one of the additional methods which could be used to classify patients into groups, which could ease the identification of the underlying gene defect, or which could support positional cloning by narrowing down the list of candidate genes. The application of microarray based mutation detection and gene expression analysis for OXPHOS disorders will be the basis of this thesis.

\section{Microarrays}

Especially since the completion of the working draft of the human genome DNA sequence [25, 26], the use of microarray technology has been emerging fast as an extremely useful tool for gene expression studies, genotyping, and resequencing at "a whole genome" level and in a high throughput manner. For gene expression studies, the microarray technology makes use of complementary DNA (cDNA) or oligonucleotide probes which hybridise to the gene transcript, the messenger RNA (mRNA) (figure 3). Probes are printed or synthesised on a solid support, i.e. a microarray slide or gene chip, forming probe spots of which each represents a transcript of a gene. After extraction of RNA from two sample groups, the target (RNA) of each group is converted to CDNA and for a 2-colour microarray, labelled with different fluorescent dyes, $\mathrm{Cy}_{3}$ (green) for one group and $\mathrm{Cy}_{5}$ (red) for the other group. The labelled targets are then combined in equal amounts and hybridised to the microarray where each labelled target will bind to its complementary probe. After hybridisation, the microarray is stringently washed to remove aspecific bound targets and scanned in a microarray scanner. The scanner excites the fluochromes with a laser resulting in emission of light from the dyes with different wave lengths which can be detected and measured. For example, if a gene is over-expressed in the $\mathrm{Cy}_{3}$ labelled sample compared to the cy5 labelled sample, more molecules of that sample will bind to the complementary probe compared to the other sample, resulting in a spot which will light up green; in case of under expression of the gene, it will light up red. When the expression for the gene is equal in both samples, the spot will light up yellow.

By measuring the intensities of the red and green signal for each probe spot, ratios in gene expression can be calculated between the two sample groups for each gene represented on the microarray. Besides the two-colour system described above, other microarray platforms use only one colour, and only one sample is hybridised to each microarray (figure 3). After a normalisation step between the different microarrays from one experiment, the absolute probe spot intensities and gene expression ratios can be calculated. Because tens to hundreds of thousands of probes can be spotted or synthesised on one microarray slide or chip, it is possible to create microarrays containing the whole human transcriptome, enabling the measurement of the expression level of all human genes in one experiment simultaneously. This allows for characterisation of gene expression signatures that can classify specific disease conditions or for identification and understanding of molecular biological pathways or single genes involved in disease or pathology. 
One of the earlier reports on cancer classifications used DNA microarrays to distinguish between acute myeloid leukaemia and acute lymphoblastic leukaemia by a gene expression class predictor [27]. In an other study, a 'poor prognosis' gene expression signature was identified to predict the risk of breast cancer metastasis [28]. Mootha et al. used the microarray gene expression technology in combination with a specific analysis technique focussing on groups of functionally related genes and identified a number of genes involved in oxidative phosphorylation which were under-expressed in human diabetic muscle [29]. This latter study illustrated the additive value of focussing on gene groups or pathways besides focussing on significant differentially expressed genes only.

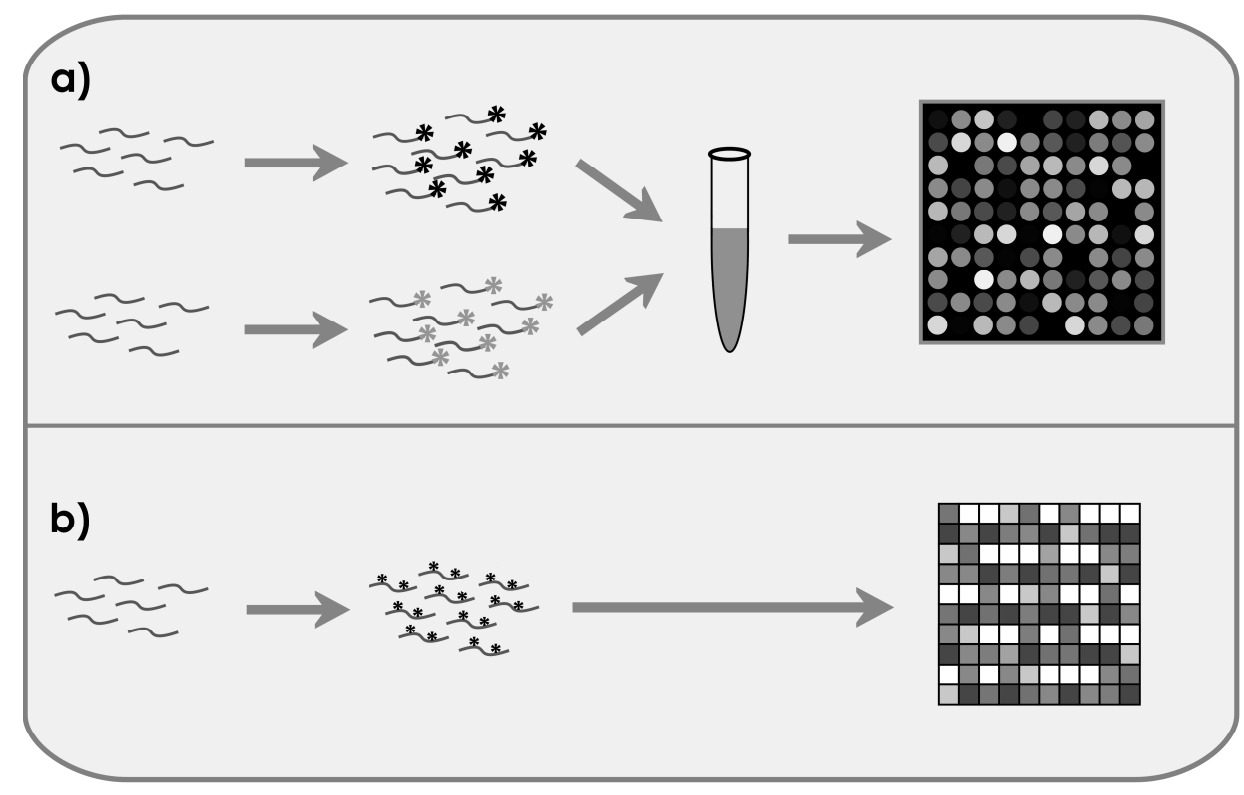

Figure 3: Microarray gene expression technique

(a) 2 colour microarray experiment: RNA from two samples is converted to CDNA and labelled with different fluorescent dyes (Cy3/green or Cy5/red). Labelled targets are subsequently co-hybridised to the microarray slide. Absolute and relative intensities are retrieved for each colour by scanning the slide in a microarray scanner. When a red spot indicates up-regulation, green will indicate down-regulation and yellow indicates no change; (b) Single colour microarray experiment: only one RNA sample is amplified, labelled and hybridised to the microarray slide. Absolute intensities are retrieved by scanning the slide in a microarray scanner. Relative intensities have to be computed by comparing intensities with other slides.

The image was taken from Eijssen [30].

As illustrated by the examples above, microarrays have already proven their value in understanding pathological processes in complex diseases like cancer and diabetes. Therefore, we explored the potential of this technology for the study of OXPHOS disorders. As a class prediction tool it could help in facilitating diagnosis by classifying patients, reducing the number of candidate genes, and specifying the candidate genes for mutation screening. One of the possibilities would be to identify a gene expression classification signature which could distinguish between OXPHOS disorders due to mtDNA or nDNA mutations. Additionally, more specific classification categories could be set, e.g. to identify whether mutations reside in genes 
coding for structural components of the OXPHOS system, or in genes which are involved in mitochondrial biogenesis. Ideally, a gene expression classification would have to be identified which would be able to identify the type of OXPHOS disorder in combination with a specific genetic defect, e.g. MELAS with the m.3243A $>G$ or Leigh syndrome with a SURF1 mutation. The key aspect in the identification of gene expression classification signatures resides in the use of a "training" gene expression dataset coming from a first patient group to carry out supervised classification. A "test" gene expression dataset coming from a second patient group has to be generated, which is to be used to validate the established classifier. This is extremely difficult to achieve for OXPHOS disorders because of the enormous clinical and genetic heterogeneity of the patient groups. It is only possible for few conditions or genes to find homogeneous patient groups big enough to build a training and a test dataset consisting of enough samples for classification purposes. Microarray gene expression analysis is however very useful to identify molecular biological processes or gene groups involved in the onset and progression of OXPHOS disorders. As there are no established therapies for OXPHOS disorders at the moment, this will provide valuable information for understanding OXPHOS pathology and subsequently for the development of possible treatments.

\section{Aims and contents of this thesis}

OXPHOS is under dual genetic control of the nDNA and the mtDNA. OXPHOS disorders are clinically and genetically heterogeneous, which makes it difficult to determine the genetic defect and explain the pathology, and symptom-based protocols which link clinical symptoms directly to a specific gene or mtDNA mutation are often falling short. Therefore, we addressed the use of microarray gene expression profiling as a tool for classifying patients or genetic defects. As it turned out that classification was difficult, due to the genetic heterogeneity of the diseases involved, we added a chip-based approach for high-throughput screening of the mtDNA. Gene expression profiling was additionally performed to get further insight in the pathogenic molecular mechanisms involved in patients.

The aims of this thesis are:

- To develop new approaches which facilitate genetic diagnosis of OXPHOS disorders.

- To understand primary and secondary pathological molecular processes that are involved in OXPHOS disorders.

- To characterise novel therapeutic entries for the treatment of OXPHOS disorders.

Chapter two describes the use of a microarray based method to resequence the entire mtDNA in order to improve and speed up genetic diagnosis of OXPHOS disorders. A quarter of the mainly paediatric OXPHOS patients could be genetically diagnosed using this technique. The usefulness of this technique as a diagnostic tool will be discussed and a comparison will be made with other new rapidly developing mutation detection techniques. Chapters three, four and five present 
microarray gene expression studies in skeletal muscle and cultured primary skin fibroblasts from OXPHOS patients in an effort to elucidate primary and secondary molecular biological pathways involved in the onset and persistence of these disorders. These pathways were evaluated to provide possible new therapeutic entries for OXPHOS disorders. A number of novel, but also known molecular processes involved in OXPHOS disorders were identified. The experimental models, the processes identified and their contribution to the identification of new therapeutic entries for the treatment of OXPHOS disorders are discussed in chapter six. 



\section{CHAPTER 2}

\section{Chip-based mtDNA mutation screening enables fast and reliable genetic diagnosis of OXPHOS patients}

R.G.E. van Eijsden ${ }^{1,2, \wedge}$, M. Gerards ${ }^{2,3,}{ }^{\wedge}$, L.M.T. Eijssen ${ }^{3,4}$, A.T.M. Hendrickx ${ }^{1}$, R.J.E. Jongbloed ${ }^{1,2,4}$, J.H.J. Wokke ${ }^{5}$, R.Q. Hintzen ${ }^{6}$, M.E. Rubio-Gozalbo ${ }^{7}$, I.F.M. de $\mathrm{Coo}^{6}$, E. Briem ${ }^{8}$, V. Tiranti ${ }^{8}$, H.J.M. Smeets ${ }^{1,2,3}$

'Department of Clinical Genetics, Maastricht University, The Netherlands.

${ }^{2}$ Research Institute Growth and Development (GROW), Maastricht University, The Netherlands.

${ }^{3}$ Department of Population Genetics, Genomics and Bioinformatics, Maastricht University, The Netherlands.

${ }^{4}$ Cardiovascular Research Institute Maastricht (CARIM), Maastricht University, The Netherlands.

${ }^{5}$ Department of Neurology, Rudolf Magnus Institute of Neuroscience, University Medical Centre Utrecht, Utrecht, The Netherlands.

${ }^{6}$ Department of Neurology, Erasmus MC, Rotterdam, The Netherlands.

${ }^{7}$ Department of Paediatrics and Laboratory Genetic Metabolic Diseases, Maastricht University Hospital, Maastricht, The Netherlands.

${ }^{8}$ Pierfranco and Luisa Mariani Centre for the Study of Children's Mitochondrial Disorders National Neurological Institute "C. Besta," Unit of Molecular Neurogenetics, Milan, Italy.

`These authors contributed equally to this work.

Genetics In Medicine 2006; 8 (10): 620-627 


\begin{abstract}
Purpose: Oxidative phosphorylation (OXPHOS) is under dual genetic control of the nuclear and the mitochondrial DNA (mtDNA). OXPHOS disorders are clinically and genetically heterogeneous, which makes it difficult to determine the genetic defect, and symptom-based protocols which link clinical symptoms directly to a specific gene or mtDNA mutation are falling short. Moreover, approximately $25 \%$ of the paediatric patients with oxidative phosphorylation disorders is estimated to have mutations in the mtDNA and a standard screening approach for common mutations and deletions will only explain part of these cases. Therefore, we tested a new chipbased screening method for the mtDNA. Methods: MitoChip (Affymetrix) resequencing was performed on three test samples and on 28 patient samples. Results: Call rates were $94 \%$ on average and heteroplasmy detection levels varied from 5 to $50 \%$. A genetic diagnosis can be made in almost a quarter of the patients at a potential throughput of 8 complete mtDNA sequences per 4 days. Moreover, a number of potentially pathogenic unclassified variants (UVs) were detected. Conclusions: The availability of long-range PCR protocols and the predominance of single nucleotide substitutions in the mtDNA make the resequencing chip a very fast and reliable method to screen the complete mtDNA for mutations.
\end{abstract}

\title{
Introduction
}

Oxidative phosphorylation (OXPHOS) disorders affect at least 1 in 8000 of the general population and belong to the group of most common inherited metabolic diseases [31]. Disease manifestations due to OXPHOS defects can be highly variable, but usually involve tissues with a high energy demand like heart, muscle, renal and the endocrine systems [32]. Several welldescribed syndromes are known, like Kearns-Sayre syndrome (KSS) and Pearson syndrome, Neuropathy Ataxia Retinitis Pigmentosa (NARP), Mitochondrial Encephalomyopathy, Lactic Acidosis and Stroke-like episodes (MELAS) and Myoclonus Epilepsy and Ragged Red Fibres (MERRF). However, OXPHOS defects can also manifest with more common and less specific symptoms, like type 2 diabetes, deafness, encephalopathy, myopathy, and cardiomyopathy. OXPHOS disorders therefore cause significant morbidity and mortality and have a broad impact on public health.

Approximately $25 \%$ of the paediatric patients with OXPHOS disorders have mutations in the mtDNA, but these are difficult to find due to the genetic and clinical heterogeneity [23]. As the number of mtDNA mutations has increased to over 250 and the clinical specificity lags behind, symptom-based protocols fall short and screening of the entire mtDNA is preferable [22]. The recently introduced MitoChip (Affymetrix) is a new method for mtDNA resequencing [33, 34]. In this paper we describe our experience of the complete mtDNA screening of 28 patients with OXPHOS disease by MitoChip resequencing. 


\section{Materials \& methods}

\section{Patient and test samples}

Genomic DNA was isolated from muscle according to the protocol of Mullenbach et al. [35]. DNA from 3 conventionally sequenced patients, a patient with a heteroplasmic 5 bp deletion [36] and 28 patients with a clinical and/or biochemical phenotype of OXPHOS disease were analysed on the MitoChip. These patients were all negative for the MELAS m.3243A>G, MERRF m.8344A>G, and NARP m.8993T >C/G mutations, and for large deletions of the mtDNA.

\section{MitoChip and experimental procedure}

The MitoChip contains both sense and anti-sense probes specific for the forward and reverse sequence of two fragments of the mtDNA. One fragment interrogates 15,446 bases of the mtDNA minus the D-loop sequence. The other fragment is a duplicate of the first fragment, comprising 12,938 bases of the mtDNA coding sequence minus the sequence of the $12 \mathrm{~S}$ and $16 \mathrm{~S}$ mitochondrial ribosomal RNA genes. The two fragments are separated on the chip by a control fragment (Affymetrix). Oligonucleotide probes are synthesised in situ on the chip by Affymetrix by standard photolithography and solid-phase DNA synthesis [37, 38]. For each position of the mtDNA fragments, four 25-mer probes are represented on the chip, each with a different nucleotide in the middle $(A, G, C$, or $T)$ allowing for the detection of all possible nucleotide substitutions. The probe with the correct corresponding nucleotide in the middle for each mtDNA position will give the highest signal intensity after hybridisation and scanning. The entire mtDNA was amplified using the Expand Long Template system (Roche) in 2 fragments ( $A$ and B) of 8466 bp and 7866 bp in length, with an overlap of 235 bp. The primers for fragment $A$ were forward primer [5'ccgcttctggccacagcacttaaacacatc-3'] and reverse primer [5'-aggaggttagttgtggcaat-3'], and for fragment B forward primer [5'-gcttcattcattgcccccac-3'] and reverse primer [5'ggaggatggtggtcaagggacccctatctg-3']. The $7.5 \mathrm{~kb}$ control sequence was amplified using the primers and template from the Customseq ${ }^{\mathrm{TM}}$ control kit (Affymetrix). PCR products were purified using the QIAQuick PCR cleanup kit (QIAGEN). Equimolar amounts of the amplified fragments A and $B$ were pooled and fragmented together with the amplified $7.5 \mathrm{~kb}$ control sequence, labelled and hybridised on a pre-hybridised MitoChip as described in the Affymetrix CustomSeq Resequencing protocol. Chips were washed and stained on the GeneChip fluidics station 400 (Affymetrix) using the pre-programmed CustumSeq Resequencing wash and stain protocol (DNA ARRAY-WS2). The MitoChips were scanned using the Affymetrix GeneChip scanner 3000 creating .CEL files for subsequent analysis.

\section{Data analysis}

Affymetrix GeneChip DNA Analysis Software (GDAS) version 3.0.1.3 beta was used to analyse the .CEL files, using the default program settings. A report file was created by GDAS listing the nucleotide variations for both chip fragments compared to the Revised Cambridge Reference 
Sequence (RCRS) [22, 39]. Discrepancies between nucleotide calls for both chip fragments were evaluated manually. The final base changes were written to a text file ('affy output file'). A second analysis was performed using $R$, a free software environment for statistical computing and graphics [40]. In the R analysis, each chip (.CEL file) was analysed separately. For each position the nucleotide with the highest signal intensity was determined, not taking into account the background signal. Nucleotide changes were printed to a text file ('R output file'). Finally the 'affy output file' and the ' $\mathrm{R}$ output file' were compared and combined, resulting in a list of variations for each sample. Discrepancies between the GDAS and R output were evaluated manually by directly looking at the chip image or by conventional sequencing. Unless otherwise mentioned, throughout this paper the phrase 'MitoChip analysis' refers to the combined GDAS and R analysis.

\section{Validation of variations and heteroplasmy levels}

Both strands of a fragment carrying a variant were cycle sequenced using the BigDye Terminator v3.1 Cycle sequencing kit, an ABI- PRISM 3100 genetic analyser and the Sequence Analysis 3.7 software package. The level of heteroplasmy was determined by mutation specific restriction digestion. In case a restriction site was gained or lost, fragments were amplified using primers surrounding the underlying variation, otherwise a specific mismatch primer was designed in order to create a restriction site. On PCR amplification, a labelled primer was added to the reaction in the last PCR cycle to label the PCR products. Labelled PCR products were digested and the fragments were analysed on an ABI-PRISM 3100 genetic analyser using the GeneScan Analysis 3.7 software package (Applied Biosystems). The level of heteroplasmy was determined by calculating the ratio of the mutant or wild type peak area (depending on the gain or loss of a restriction site by the nucleotide variation) and the sum of the mutant and wild type peak areas. Primer sequences and reaction conditions are available on request.

\section{Results}

\section{MitoChip performance and validation}

Based on a total of 32 DNA samples, the average GDAS call rate was $94.0 \%$, ranging from $88.9 \%$ to 96.8\%. Of the GDAS 'no call' signals, 0.72\% (212/29366) of the chip positions gave a 'no call' for each of the 32 chips analysed, corresponding to $134 \mathrm{mtDNA}$ nucleotide positions. A GDAS call was always given for each of the 32 chips for 12200 mtDNA nucleotide position (20486 out of 29366 (70\%) chip positions). For 7 chips GDAS and $\mathrm{R}$ analysis were compared for the entire sequence and $R$ analysis accounted for an average of $24.2 \%$ (ranging from $13 \%$ to $37 \%$ ) of the total amount of base changes in the output files. Five unclassified variants (UVs) were identified by the $R$ analysis, which were not detected by the standard GDAS analysis. For the three test samples, which were also sequenced conventionally, 34 out of 35 (97\%) variations were called correctly on the MitoChip. In two test samples, one and six variations were additionally detected by the MitoChip. By reinterpretation of the conventional sequencing results, one of the variations which were detected additionally by the MitoChip proved to be a low percentage heteroplasmic substitution 
(false negative), and the other additional variants appeared to be falsely detected as a nucleotide variant by the MitoChip (false positives). A known 5 bp deletion was also tested, but not detected. Two DNA samples differing at 16 positions in their mtDNA sequence were mixed at ratios of 1:1, 1:9 and 1:19. In the mix sample analyses (only GDAS), the Total Quality Threshold Score setting was set at 30 and 75. Fifteen of sixteen positions were called correctly at a heteroplasmy level of $50 \%$ for both TQT settings. Only one position was called correctly as heteroplasmic in all three mix samples at both TQT settings. At a TQT value of 30, 'no call' signals were 3 times more often observed at the 16 investigated heteroplasmy positions than at a TQT value of 75 .

mtDNA variations in 28 patients

A total of 520 variations were detected in 28 patients, comprising 197 unique nucleotide substitutions. Fifteen heteroplasmic variations were detected in a total of 11 patients. Four of these variations were confirmed by sequencing and mutation specific restriction digestion ( $\mathrm{m} .15939 \mathrm{C}>\mathrm{T}$ at $7 \%, \mathrm{~m} .13513 \mathrm{G}>\mathrm{A}$ at $14 \%, \mathrm{~m} .3243 \mathrm{~A}>\mathrm{T}$ at $34 \%$, and $\mathrm{m} .13042 \mathrm{G}>\mathrm{A}$ at $84 \%$ ); two proved to be homoplasmic single base pair insertions (m.3229_3230insA and m.3158_3159insT); two appeared to be false positive; one was shown to be a homoplasmic polymorphism (m.15452C $>A$ ); the remaining six were not tested because they were considered not to be pathogenic. Of all detected variations, three were known pathogenic mutations (table 1), 114 have been reported before as polymorphisms (supplementary table S1), 41 did not result in an amino acid change and were most likely polymorphisms (supplementary table S1), and 39 were unclassified variants (UVs), some of which were likely pathogenic (table 2). Eight variants were located in tRNA molecules (figure 1). In three patients known pathogenic mutations were detected and in 23 patients UVs were detected. In five patients only polymorphisms were detected.

UVs were evaluated for evolutionary conservation, for functional significance by determining the effect on the tRNA or on the protein, and - when available - for segregation in the family. Seven UVs were located in six tRNA genes (figure 1), 11 UVs in the $12 \mathrm{~S}$ and $16 \mathrm{~S}$ rRNA genes, one UV in the mtDNA transcription terminator site, and 20 UVs in the protein encoding genes. Of the eight tRNA variations (figure 1), the m.15939C $>\mathrm{T}$ variation in the tRNA-Thr gene was heteroplasmic with a mutation load of $7 \%$. The other tRNA variations were all homoplasmic or close to homoplasmy (>98\% mutation load). Two of the variations in the protein encoding genes were heteroplasmic $84 \%$ and $70 \%$, respectively, for the $\mathrm{m} .13042 \mathrm{G}>\mathrm{A}$ in the ND5 gene and $\mathrm{m} .14258 \mathrm{G}>\mathrm{A}$ in the ND6 gene.

Single nucleotide insertions were detected as well. A heteroplasmic m.3158A $>$ T transition on the chip proved to be a single nucleotide insertion (m.3158_3159insT) by standard sequencing. Additionally, the homoplasmic m.3229_3230insA insertion was initially detected by the MitoChip analysis as a heteroplasmic m.3229T>A transition. 
Table 1: Evident pathogenic mutations

\begin{tabular}{|c|c|c|c|c|c|c|c|c|c|}
\hline Position & $\begin{array}{l}\text { nt } \\
\text { change }\end{array}$ & $\begin{array}{l}\text { aa } \\
\text { change }\end{array}$ & $\begin{array}{l}\text { Mutation } \\
\text { load * }\end{array}$ & Locus & Disease ** & $\begin{array}{l}\text { No. of } \\
\text { samples }\end{array}$ & $\begin{array}{l}\text { aa / n } \\
\text { Conservation *** }\end{array}$ & $\begin{array}{l}\text { No. patients } \\
\text { previously } \\
\text { described }\end{array}$ & Reference(s) \\
\hline 3243 & $A>T$ & - & $34 \%$ & tRNA-Leu1 & MM & 1 & 30/31 species & 1 & $\begin{array}{l}\text { Shaag et al. } \\
\text { (1997) [41] }\end{array}$ \\
\hline 3697 & $G>A$ & G-S & $>97 \%$ & ND1 & MELAS & 1 & $\begin{array}{l}\mathrm{Hs} / \mathrm{Pt} / \mathrm{Cf} / \mathrm{Mm} / \mathrm{Rn} / \\
\mathrm{Gg} / \mathrm{Dm} / \mathrm{Ag} / \mathrm{Ce} / \mathrm{At} / \mathrm{Os}\end{array}$ & 1 & $\begin{array}{l}\text { Kirby et al. } \\
\text { (2004) [42] }\end{array}$ \\
\hline \multirow[t]{6}{*}{13513} & $G>A$ & D-N & $13-15 \%$ & ND5 & $\begin{array}{l}\text { MELAS / } \\
\text { Leigh Disease }\end{array}$ & 1 & $\begin{array}{l}\mathrm{Hs} / \mathrm{Pt} / \mathrm{Cf} / \mathrm{Mm} / \mathrm{Rn} / \\
\mathrm{Gg} / \mathrm{Dm} / \mathrm{Ag} / \mathrm{Ce} / \mathrm{At} / \mathrm{Os}\end{array}$ & 1 & $\begin{array}{l}\text { Santorelli et al. } \\
\text { (1997) [43] }\end{array}$ \\
\hline & & & & & & & & 4 & $\begin{array}{l}\text { Pulkes et al. } \\
\text { (1999) [44] }\end{array}$ \\
\hline & & & & & & & & 1 & $\begin{array}{l}\text { Penisson-Besnier et al. } \\
(2000) \text { [45] }\end{array}$ \\
\hline & & & & & & & & 3 & $\begin{array}{l}\text { Chol et al. } \\
(2003) \text { [46] }\end{array}$ \\
\hline & & & & & & & & 3 & $\begin{array}{l}\text { Kirby et al. } \\
\text { (2003) [47] }\end{array}$ \\
\hline & & & & & & & & 6 & $\begin{array}{l}\text { Sudo et al. } \\
\text { (2004) [48] }\end{array}$ \\
\hline
\end{tabular}

* Mutation loads are determined by mutation specific restriction digestion analysis followed by GeneScan analysis.

** MM: Mitochondrial Myopathy; MELAS = Mitochondrial Encephalomyopathy, Lactic Acidosis, and Stroke-like episodes.

*** For the tRNA variations, the nucleotide conservation is shown. For the variations in protein encoding genes, the amino acid conservation is displayed. Hs: Homo sapiens, Pt: Pan troglodytes, Cf: Canis familiaris, Mm: Mus musculus, Rn: Rattus norvegicus, Gg: Gallus gallus, Dm: Drosophila melanogaster, Ag: Anopheles gambiae, Ce: Caenorhabditis elegans, At: Arabidopsis thaliana, Os: Oryza sativa.

tRNA conservations were taken from the website dealing with the compilation of mammalian tRNA genes (http://mamit-trna.ustrasbg.fr/index.html) [49].

\section{Discussion}

\section{MitoChip performance and validation}

MitoChip resequencing is a very rapid method to screen the mtDNA for mutations. Eight samples can be analysed by one technician over a period of 4 days. The average call rate of $94.0 \%$ is within the range described by Affymetrix and others [33]. The additional $R$ analysis led to an increase in total base calls and to the identification of 5 UVs (in 4 out of 7 samples), which would have been missed by GDAS analysis only. Of the 212 chip positions ( $0.72 \%$ ) (134 mtDNA nucleotide positions) which consistently gave a 'no call', 197 (124 mtDNA nucleotide positions) were cytosine nucleotides. Previously, it was reported that $1.7 \%$ of the bases always gave a 'no call' in a dataset of 26 chips [33]. About $51 \%$ of these were from regions containing two or more successive $C$ bases. $\mathrm{R}$-analysis showed that in these regions especially the forward strand differed from the reference sequence, whereas the reverse sequence gave a call equal to the reference sequence. The reason for this is yet unknown. The mtDNA nucleotide positions of twelve common mtDNA mutations (m.1555A $>$ G, m.3243A $>$ G, m.3460G $>A, m .8344 A>C, m .8993 T>G / C . m .9176 T>C, m .10159 T>C$, $m .11778 G>A, m .13513 G>A, m .14459 G>A, m .14484 T>C$, and $m .14487 T>C$ ), were not among the 
nucleotide positions always giving a 'no-call'. Within-chip and between-chip reproducibility have already been determined showing a within-chip error rate of $0.0025 \%$ and a between-chip error rate of $0.0027 \%$, illustrating that the base calls were reproducible for $>99.99 \%$ [33]. In our experiments $97 \%$ of the variations was called correctly in three previously sequenced samples, which is comparable with the $95 \%$ reported before in 18 samples [33]. In the three test samples, only six variations appeared be false positive after follow-up investigation. It is unlikely that PCR errors are causative for false positive MitoChip calls since there is a large number of templates for the PCR, and the heteroplasmy levels due to sporadic PCR errors will be below the detection limit. As GeneChip Resequencing Arrays only support detection of homozygous and heterozygous single nucleotide substitutions, small deletions ( $5 \mathrm{bp}$ ) could not be detected, although 2 single nucleotide insertions were detected as heteroplasmic "substitutions". One might expect that the heteroplasmic 5 bp deletion would be detected by the MitoChip as a loss of signal, but available software is insufficient for these quantitative analyses, especially if over $50 \%$ of the wild type mtDNA is still present [36].

Although the GDAS software was designed to detect homozygous (homoplasmic) and heterozygous ( $50 \%$ heteroplasmy) single base pair substitutions only, it was previously shown that heteroplasmic variations at levels as low as $2 \%$ were detectable [33]. However, in our dilution experiments only one out of 16 positions could be identified correctly as a heteroplasmic variation in the $5 \%, 10 \%$, and $50 \%$ mix samples. Notably, the number of 'no calls' at these 16 heteroplasmic positions was three times higher at the TQT setting of 30 compared to the GDAS default TQT setting of 75 , illustrating a difficulty for the algorithm to assign a call for the particular position, probably because of the signal of the heteroplasmic variant. This suggests that further development of the data analysis algorithm and tuning of the data analysis settings can improve the detection level of heteroplasmic mutations. An option would be to customise our R-analysis to make it sensitive for heteroplasmic variations. Nevertheless, we were able to detect a heteroplasmic variation in the tRNA-Thr gene $(\mathrm{m} .15939 \mathrm{C}>\mathrm{T})$ in a patient with a mutation load as low as $7 \%$. Additionally, a pathogenic mutation in the ND5 gene $(\mathrm{m} .13513 \mathrm{G}>\mathrm{A})$ was identified and validated at a heteroplasmic level of $13 \%-15 \%$. Apparently, the sensitivity for heteroplasmy detection is different per position and probably depends on the sequence surrounding the variation. 
Table 2: Unclassified Variants

\begin{tabular}{|c|c|c|c|c|c|c|}
\hline \multicolumn{7}{|c|}{ non-protein-coding region, unclassified variants } \\
\hline Position & $\begin{array}{l}\text { nt } \\
\text { change }\end{array}$ & $\begin{array}{l}\text { aa } \\
\text { change }\end{array}$ & $\begin{array}{l}\text { Mutation } \\
\text { load * }\end{array}$ & Locus & $\begin{array}{l}\text { No. of } \\
\text { samples }\end{array}$ & nt Conservation ** \\
\hline 892 & $A>T$ & - & $\mathrm{h}$ & 12S rRNA & 3 & - \\
\hline 1860 & $A>G$ & - & $100 \% \$$ & 16S rRNA & 1 & - \\
\hline 2098 & $G>A$ & - & $100 \% \$$ & 16S rRNA & 1 & - \\
\hline 2259 & $\mathrm{C}>\mathrm{T}$ & - & $100 \% \$$ & 16S rRNA & 1 & - \\
\hline 2361 & $\mathrm{G}>\mathrm{A}$ & - & $100 \% \$$ & 16S rRNA & 1 & - \\
\hline 2581 & $A>G$ & - & $100 \% \$$ & 16S rRNA & 1 & - \\
\hline 2757 & $A>G$ & - & $100 \% \$$ & 16S rRNA & 1 & - \\
\hline 2768 & $A>G$ & - & $100 \% \$$ & 16S rRNA & 2 & - \\
\hline 2825 & $\mathrm{G}>\mathrm{C}$ & - & $\mathrm{h}$ & 16S rRNA & 4 & - \\
\hline 3105 & $A>G$ & - & $100 \% \$$ & 16S rRNA & 1 & - \\
\hline 3159 & insT & - & $100 \% \$$ & 16S rRNA & 1 & - \\
\hline 3229 & insA & - & $\mathrm{h}$ & Transcription terminator & 1 & - \\
\hline 4336 & $\mathrm{~T}>\mathrm{C}$ & - & $>98 \%$ & tRNA-GIn & 2 & 13/31 species \\
\hline 5558 & $A>G$ & - & $100 \%$ & tRNA-Trp & 1 & $31 / 31$ species \\
\hline 5592 & $A>G$ & - & $100 \%$ & tRNA-Ala & 1 & 28/31 species \\
\hline 5850 & $\mathrm{~T}>\mathrm{C}$ & - & $>98 \%$ & tRNA-Tyr & 1 & $30 / 31$ species \\
\hline 12308 & $A>G$ & - & $100 \% \$$ & tRNA-Leu2 & 5 & $31 / 31$ species \\
\hline 15890 & $C>T$ & - & $100 \%$ & tRNA-Thr & 1 & $31 / 31$ species \\
\hline 15939 & $C>T$ & - & $7 \%$ & tRNA-Thr & 1 & 13/31 species \\
\hline
\end{tabular}

\begin{tabular}{|c|c|c|c|c|c|c|c|}
\hline \multicolumn{8}{|c|}{ protein coding region, unclassified variants } \\
\hline Position & $\begin{array}{l}\text { nt } \\
\text { change }\end{array}$ & $\begin{array}{l}\text { aa } \\
\text { change }\end{array}$ & $\begin{array}{l}\text { Mutation } \\
\text { load * }\end{array}$ & Locus & $\begin{array}{l}\text { No. of } \\
\text { samples }\end{array}$ & aa Conservation ** & Pathogenicity score ${ }^{\wedge}$ \\
\hline 3308 & $\mathrm{~T}>\mathrm{C}$ & $M>T$ & $100 \% \$$ & ND1 & 2 & $\mathrm{Hs} / \mathrm{Cf} / \mathrm{Mm} / \mathrm{Rn} / \mathrm{Ag}$ & $0(28)$ \\
\hline 4501 & $C>T$ & $S>F$ & $100 \% \$$ & ND2 & 1 & $\mathrm{Hs} / \mathrm{Cf}$ & $0(20)$ \\
\hline 4561 & $\mathrm{~T}>\mathrm{C}$ & $V>A$ & $100 \% \$$ & ND2 & 1 & $\mathrm{Hs} / \mathrm{Pt} / \mathrm{Mm} / \mathrm{Rn} / \mathrm{At} / \mathrm{Os}$ & $0(20)$ \\
\hline 5319 & $A>G$ & $\mathrm{~T}>\mathrm{A}$ & $100 \% \$$ & ND2 & 1 & $\mathrm{Hs}$ & $0(20)$ \\
\hline 6408 & $A>G$ & $\mid>V$ & $100 \% \$$ & C01 & 1 & $\mathrm{Hs} / \mathrm{Pt} / \mathrm{Cf} / \mathrm{Mm} / \mathrm{Rn} / \mathrm{Gg} / \mathrm{Dm} / \mathrm{Ag} / \mathrm{Sp}$ & $6(20)$ \\
\hline 7146 & $A>G$ & $\mathrm{~T}>\mathrm{A}$ & $100 \% \$$ & C01 & 2 & $\mathrm{Hs} / \mathrm{Cf}$ & $0(28)$ \\
\hline 7389 & $\mathrm{~T}>\mathrm{C}$ & $\mathrm{Y}>\mathrm{H}$ & $100 \% \$$ & C01 & 2 & $\mathrm{Hs} / \mathrm{Pt}$ & $0(28)$ \\
\hline 8516 & $\mathrm{~T}>\mathrm{C}$ & $W>R$ & $100 \% \$$ & ATP8 & 1 & $\mathrm{Hs} / \mathrm{Pt} / \mathrm{Mm} / \mathrm{Rn}$ & $7(23)$ \\
\hline 8975 & $\mathrm{~T}>\mathrm{C}$ & $L>P$ & $100 \% \$$ & ATP6 & 1 & $\mathrm{Hs} / \mathrm{Pt} / \mathrm{Gg}$ & $4(20)$ \\
\hline 10680 & $G>A$ & $A>T$ & $100 \% \$$ & ND4L & 1 & $\mathrm{Hs} / \mathrm{Pt} / \mathrm{Cf} / \mathrm{Mm} / \mathrm{Rn}$ & $15(28)$ \\
\hline 11447 & $G>A$ & $\mathrm{~V}>\mathrm{M}$ & $100 \% \$$ & ND4 & 1 & $\mathrm{Hs} / \mathrm{Pt} / \mathrm{Cf}$ & $2(20)$ \\
\hline 13042 & $G>A$ & $A>T$ & $84 \%$ & ND5 & 1 & $\mathrm{Hs} / \mathrm{Pt} / \mathrm{Cf} / \mathrm{Mm} / \mathrm{Rn} / \mathrm{Gg} / \mathrm{Dm} / \mathrm{Ag} / \mathrm{Ce} / \mathrm{At} / \mathrm{Os}$ & $18(31)$ \\
\hline 13630 & $A>G$ & $\mathrm{~T}>\mathrm{A}$ & $100 \% \$$ & ND5 & 1 & $\mathrm{Hs} / \mathrm{Pt} / \mathrm{Gg} / \mathrm{Dm} / \mathrm{Ag}$ & $8(28)$ \\
\hline 13880 & $C>A$ & $S>Y$ & $100 \% \$$ & ND5 & 2 & $\mathrm{Hs} / \mathrm{Pt} / \mathrm{At}$ & $0(28)$ \\
\hline
\end{tabular}




\begin{tabular}{llllllll}
14207 & $\mathrm{G}>\mathrm{A}$ & $\mathrm{T}>\mathrm{I}$ & $100 \% \$$ & $\mathrm{ND6}$ & 1 & $\mathrm{Hs} / \mathrm{Pt} / \mathrm{Cf}$ & $8(28)$ \\
14258 & $\mathrm{G}>\mathrm{A}$ & $\mathrm{P}>\mathrm{L}$ & $70 \% \$ \$$ & $\mathrm{ND6}$ & 1 & $\mathrm{Hs} / \mathrm{Pt}$ & $5(20)$ \\
14766 & $\mathrm{C}>\mathrm{T}$ & $\mathrm{T}>\mathrm{I}$ & $100 \% \$$ & $\mathrm{CYB}$ & 13 & $\mathrm{Hs} / \mathrm{Pt}$ & $8(28)$ \\
15311 & $\mathrm{~A}>\mathrm{G}$ & $\mathrm{I}>\mathrm{V}$ & $100 \% \$$ & $\mathrm{CYB}$ & 1 & $\mathrm{Hs} / \mathrm{Pt} / \mathrm{Cf} / \mathrm{Mm} / \mathrm{Rn} / \mathrm{Gg} / \mathrm{Ag} / \mathrm{Ce} / \mathrm{Sp} / \mathrm{Sc} / \mathrm{K} / \mathrm{Eg}$ & $1(23)$ \\
15725 & $\mathrm{C}>\mathrm{T}$ & $\mathrm{L}>\mathrm{F}$ & $100 \% \$$ & $\mathrm{CYB}$ & 1 & $\mathrm{Hs} / \mathrm{Pt} / \mathrm{Cf} / \mathrm{Gg} / \mathrm{K} / \mathrm{Eg} / \mathrm{At}$ & $2(20)$ \\
15824 & $\mathrm{~A}>\mathrm{G}$ & $\mathrm{T}>\mathrm{A}$ & $100 \% \$$ & $\mathrm{CYB}$ & 1 & $\mathrm{Hs} / \mathrm{Pt} / \mathrm{Cf} / \mathrm{Gg}$ & $0(20)$ \\
\hline
\end{tabular}

* Mutation loads are determined by mutation specific restriction digestion analysis followed by GeneScan analysis.

\$ Mutations load estimated by MitoChip and/or sequencing results only.

\$ Also confirmed with denaturing high-performance liquid chromatography (dHPLC) analysis.

** Ag: Anopheles gambiae, At: Arabidopsis thaliana, Ce: Caenorhabditis elegans, Cf: Canis familiaris, Dm: Drosophila melanogaster, Eg: Eremothecium gossypii, Gg: Gallus gallus, Hs: Homo sapiens, Kl: Kluyveromyces lactis, Mm: Mus musculus, Os: Oryza sativa, Pt: Pan troglodytes, Rn: Rattus norvegicus, Sc: Saccharomyces cerevisiae, Sp: Schizosaccharomyces pombe.

$\wedge \quad$ The pathogenicity score is calculated according to the criteria set by Mitchell et al. [50]. Since information was not available on all criteria, the maximal score using only the available information is indicated between brackets.

$\mathrm{h} \quad$ Detected as heteroplasmic by MitoChip analysis.

tRNA conservations were taken from the website dealing with the compilation of mammalian tRNA genes (http://mamit-trna.ustrasbg.fr/index.html) [49].

\section{MitoChip compared to other methods}

Resequencing chips are powerful if long-range PCR protocols are available and substitutions are the predominant pathogenic mutations, which is the case for the mtDNA. Other methods for mtDNA mutation analysis are conventional sequence analysis, single strand conformation polymorphism (SSCP) analysis, denaturing gradient gel electrophoresis (DGGE), temperature gradient gel electrophoresis (TGGE), denaturant capillary electrophoresis (DCE) and denaturing high-performance liquid chromatography (dHPLC) [51-55]. These techniques all have their advantages and disadvantages with respect to costs, time consumption, high throughput possibilities, heteroplasmic detection limit, and type of mutations that can be detected (table 3). At this moment, heteroduplex based methods (dHPLC, DGGE, TGGE, and DCE) appear to be more sensitive than the MitoChip for heteroplasmy detection, with detection sensitivities as low as $0.5 \%$ $[52-54,56]$. SSCP and conventional sequence analysis are not able to detect low percentages of heteroplasmy. MitoChip resequencing is able to detect heteroplasmic variants at low levels, although the data analysis needs to be optimised to reach similar detection levels. Although the costs per nucleotide are cheaper for conventional sequencing [33], considering the overall costs per sample, MitoChip resequencing is preferable over conventional sequencing, mostly due to a tremendous gain in labour costs ( 8 samples can be analysed by one technician over a period of 4 days) and automated analysis at comparable bench costs. 

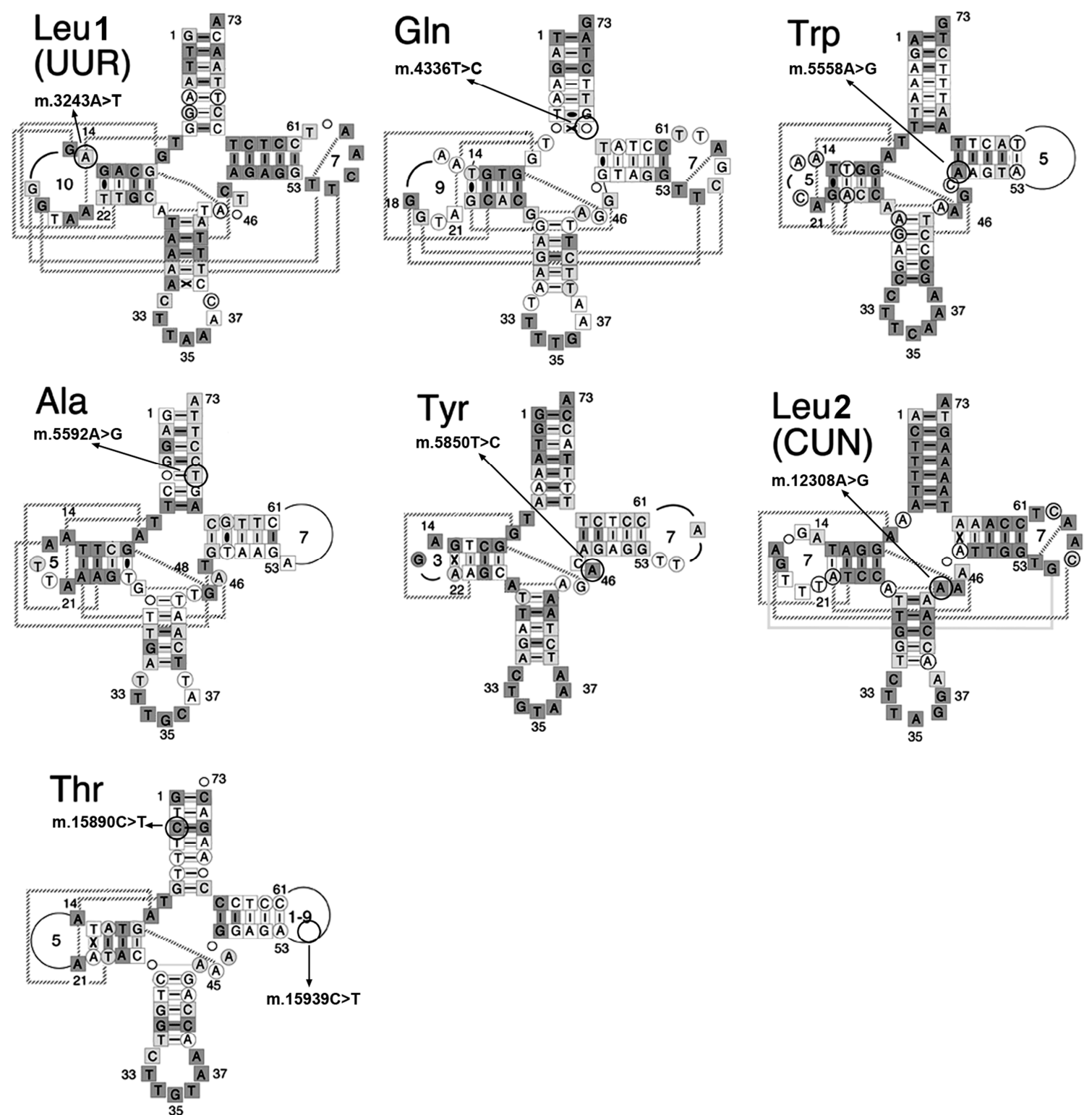

Figure 1: Eight tRNA variations (unclassified variants and mutations) detected in 28 patients with OXPHOS disease

Detected were an m.3243A > T variation in the tRNA-Leu1, an m.4336T>C variation in the tRNA-Gln gene, an m.5558A $>G$ variation in the tRNA-Trp gene, an m.5592A >G variation in tRNA-Ala gene, an m.5850T>C variation in the tRNA-Tyr gene, an m.12308A $>\mathrm{G}$ variation in the tRNA-Leu2 gene, and an m.15890C $>T$ variation and m.15939C $>$ T variation in the tRNA-Thr gene.

The images are adapted from the website dealing with the compilation of mammalian tRNA genes (http://mamit-trna.u-strasbg.fr/index.html) [49]. 
Table 3: Comparison of mtDNA mutation screening methods

\begin{tabular}{llllllll}
\hline & SSCP & DGGE & TGGE & DCE & dHPLC & Sequencing & MitoChip \\
\hline Equipment costs & low & low & low & high & high & high & high \\
Labour intensive & yes & yes & yes & no & no & yes & no \\
High throughput & - & - & - & + & + & + & ++ \\
Heteroplasmy detection & - & ++ & ++ & ++ & ++ & + & $+(+)$ \\
Homoplasmy detection & + & - & - & - & - & + & + \\
\hline
\end{tabular}

SSCP: single strand conformation polymorphism, DGGE: denaturing gradient gel electrophoresis, TGGE: temperature gradient gel electrophoresis, DCE: denaturant capillary electrophoresis, dHPLC: denaturing high-performance liquid chromatography.

\section{MtDNA variations in 28 clinical samples}

The pathogenic m.3243A>T mutation was found in a patient with proximal myopathy, ptosis and ophthalmoplegia at a mutation level of 34\%. Magnetic resonance imaging (MRI) of the brain showed white matter abnormalities and muscle creatine phosphokinase levels were elevated. Muscle biopsy histomorphology revealed ragged red fibres (RRF) and cytochrome c oxidase (COX) negative fibres. This mutation has been previously described in muscle (81.4\%), skin (69.3\%) and blood (13.8\%) of a 9-year old girl with muscle weakness, encephalopathy and a reduction of the activity of OXPHOS complexes I, III and IV [41]. The mutation is located at the same position as the classical MELAS m.A3243A>G mutation at the first position of the D-loop in the tRNA tRNALeu1 molecule (figure 1), leading to a dramatic loss of aminocylation efficiency. Moreover, residue A14 of this tRNA is strongly conserved within all classical tRNA molecules and it is involved in establishing tertiary interactions with other residues of the tRNA [57]. The pathogenic m.3697G $>A$ mutation in the ND1 gene was detected at a mutation load higher than $97 \%$ in a patient with spastic dystonia, elevated levels of lactate and pyruvate in blood serum and cerebrospinal fluid. The affected amino acid is evolutionary highly conserved (table 1) and the mutation has been described before in a MELAS patient with $80 \%$ of the $\mathrm{m} \cdot 3697 \mathrm{G}>\mathrm{A}$ mutation in muscle and $79 \%$ in skin fibroblasts [42]. Pathogenicity of the mutation was confirmed by fusion experiments with $\rho^{0}$ cell lines (no restoration of complex I activity) combined with blue native polyacrylamide gel electrophoresis (BN-PAGE) (low levels of fully assembled complex I) [42]. The m.3697G $>A$ mutations was also evaluated according to the scoring system to assess pathogenicity of complex I mutations [50]. This score can range from o (not pathogenic) to 40 (pathogenic) and is based on several criteria: biochemical defect, functional studies, reports by two or more independent laboratories, heteroplasmy, segregation in the family, and evolutionary conservation. The m.3697G $>$ A mutation reached a score of 32. Our report of this mutation as a second independent laboratory will increase the score to 37 points, which is above the "pathogenic" cut-off of 30 . The m.13513A>G mutation was detected in a patient with encephalopathy, strokes, dystonia, signs of Parkinsonism, mental retardation and signs of early fatigue. Biochemical analysis indicated a deficiency of the pyruvate dehydrogenase complex (PDHC) in muscle. The m.13513A>C transition in the ND5 gene changes an amino acid strongly conserved over 11 species (table 1) and has been related to MELAS and Leigh syndrome in several reports [43-48]. The pathogenicity score for this mutation is 39 [50]. The functional importance of this amino acid is further supported by the 
finding of a pathogenic mutation $\mathrm{m} .13514 \mathrm{~A}>\mathrm{G}$, affecting the same codon, that results in a different amino acid replacement (D393G versus D393N) in two MELAS patients [58].

UVs were evaluated with respect to heteroplasmy, evolutionary conservation, functional influence of the effect on tRNA or protein, and segregation in the family $[50,59,60]$. Several UVs were likely to be pathogenic, but the effect of others is still unclear. The m.5850T>C variation had a mutation load of $>98 \%$ in muscle and $13 \%$ in urine, but it could not be detected in blood or hair of the patient. It was not present in blood, hair, or urine from the patient's clinically unaffected mother, brother, and sister. The position of this variation in the tRNA-Tyr gene is evolutionary highly conserved. These data strongly suggest a pathogenic role. A second variation with a high probability of pathogenicity was the homoplasmic m.3229-3230insA. This variation is located in the transcription terminator site and may disturb the function of this site. Moreover, the location of this variation being exactly between the 16S rRNA gene and the tRNA-Leu1 gene might result in a faulty cleavage of the RNA after transcription. A faulty cleavage could have consequences for the function of the tRNA-Leu1 and 16S rRNA genes. The m.13042G>A variation in the ND5 gene was heteroplasmic (84\%) and the involved amino acid was also strongly conserved (table 2), which favours a pathogenic role. The m.14258G $>$ A variation in the ND6 gene was also a heteroplasmic (70\%) variation in a protein coding gene. Although this variation is heteroplasmic, the affected amino acid is not well conserved. The m.15939C $>$ T variation in the tRNA-Thr gene was also heteroplasmic (7\%) in the patient's muscle. The variation is located in the T-loop of the tRNA molecule and not involved in establishing tertiary interactions in the tRNA molecule, making a pathogenic role less likely. The other homoplasmic variations (table 2) have to be further evaluated, as it is difficult to determine pathogenicity solely based on criteria like heteroplasmy and conservation alone. Functional and family studies will be performed to provide the definite evidence.

Resequencing does not only detect known pathogenic mutations or polymorphisms, but also UVs and risk factors for unrelated pathology, like cancer, Alzheimer's disease or Parkinson's disease. As the significance of this latter group of variations for individual cases and their families is yet unclear and the risk factors can not explain the primary pathology, it is evident that these data have to be carefully dealt with by the clinicians. Patients should be counselled about the uncertainties of some of the observations and the difficulties to interpret part of the results. However, this is inevitable and does not counterweight the genetic diagnoses made. It will also be a temporary problem, as due to the joint sequencing efforts more and more information will become available about neutral polymorphisms, genuine risk factors of disease, and actual pathogenic mutations. 


\section{Conclusion}

MitoChip resequencing is a fast, cost-effective and reliable method with high throughput capabilities for complete mtDNA screening. A quarter of the patients with OXPHOS disease can be genetically diagnosed by this technique, based on the detection of three pathogenic and three or four likely pathogenic mutations in 28 patients, which confirms the percentage previously described [23]. Because of the growing number of mtDNA mutations combined with the increasing clinical heterogeneity, MitoChip resequencing is the preferred method to screen the mtDNA for mutations, especially when symptom specific screening and screening of only the most common mtDNA mutations fall short. 
Supplementary Table S1: mtDNA polymorphisms detected in 28 patients with OXPHOS disease

\begin{tabular}{|c|c|c|c|c|c|c|c|c|c|}
\hline \multicolumn{10}{|c|}{ polymorphisms - previously reported } \\
\hline Position & $\begin{array}{l}\text { nt-change } \\
\text { Chip }\end{array}$ & $\begin{array}{l}\text { nt-change } \\
\text { custom sequencing }\end{array}$ & $\begin{array}{l}\text { Heteroplasmic } \\
\text { on chip }\end{array}$ & $\begin{array}{l}\text { In No. of samples } \\
\text { heteroplasmic on chip }\end{array}$ & aa-change & Locus & No. of samples & $\%$ of samples & Population frequency * \\
\hline 709 & $g>a$ & $\mathrm{np}$ & No & - & - & MT-RNR1 / 12S ribosomal RNA & 3 & $10.7 \%$ & $17.2 \%$ \\
\hline 710 & $t>c$ & $\mathrm{np}$ & No & - & - & MT-RNR1 / 12S ribosomal RNA & 2 & $7.1 \%$ & $1.0 \%$ \\
\hline 750 & $a>g$ & $\mathrm{np}$ & No & - & - & MT-RNR1 / 12S ribosomal RNA & 27 & $96.4 \%$ & $99.1 \%$ \\
\hline 769 & $g>a$ & $\mathrm{np}$ & No & - & - & MT-RNR1 / 12S ribosomal RNA & 2 & $7.1 \%$ & $6.0 \%$ \\
\hline 825 & $t>a$ & $\mathrm{np}$ & No & - & - & MT-RNR1 / 12S ribosomal RNA & 2 & $7.1 \%$ & $3.5 \%$ \\
\hline 930 & $g>a$ & $\mathrm{np}$ & No & - & - & MT-RNR1 / 12S ribosomal RNA & 1 & $3.6 \%$ & $2.1 \%$ \\
\hline 1018 & $g>a$ & $\mathrm{np}$ & No & - & - & MT-RNR1 / 12S ribosomal RNA & 2 & $7.1 \%$ & $6.1 \%$ \\
\hline 1189 & $t>c$ & $\mathrm{np}$ & No & - & - & MT-RNR1 / 12S ribosomal RNA & 3 & $10.7 \%$ & $3.2 \%$ \\
\hline 1438 & $a>g$ & $\mathrm{np}$ & No & - & - & MT-RNR1 / 12S ribosomal RNA & 25 & $89.3 \%$ & $96.5 \%$ \\
\hline 1721 & $c>t$ & $\mathrm{np}$ & No & - & - & MT-RNR2 / 16S ribosomal RNA & 2 & $7.1 \%$ & $0.5 \%$ \\
\hline 1738 & $t>c$ & $\mathrm{np}$ & No & - & - & MT-RNR2 / 16S ribosomal RNA & 2 & $7.1 \%$ & $0.9 \%$ \\
\hline 1811 & $a>g$ & $\mathrm{np}$ & No & - & - & MT-RNR2 / 16S ribosomal RNA & 1 & $3.6 \%$ & $7.0 \%$ \\
\hline 1888 & $g>a$ & $\mathrm{np}$ & No & - & - & MT-RNR2 / 16S ribosomal RNA & 3 & $10.7 \%$ & $5.6 \%$ \\
\hline 2352 & $t>c$ & $\mathrm{np}$ & No & - & - & MT-RNR2 / 16S ribosomal RNA & 2 & $7.1 \%$ & $2.1 \%$ \\
\hline 2706 & $a>g$ & $\mathrm{np}$ & No & - & - & MT-RNR2 / 16S ribosomal RNA & 15 & $53.6 \%$ & $80.7 \%$ \\
\hline 2758 & $g>a$ & $\mathrm{np}$ & No & - & - & MT-RNR2 / 16S ribosomal RNA & 2 & $7.1 \%$ & $3.4 \%$ \\
\hline 2885 & $t>c$ & $\mathrm{np}$ & No & - & - & MT-RNR2 / 16S ribosomal RNA & 2 & $7.1 \%$ & $3.4 \%$ \\
\hline 3010 & $g>a$ & $\mathrm{np}$ & No & - & - & MT-RNR2 / 16S ribosomal RNA & 5 & $17.9 \%$ & $21.7 \%$ \\
\hline 3106 & $c>g$ & $\mathrm{np}$ & No & - & - & MT-RNR2 / 16S ribosomal RNA & 1 & $3.6 \%$ & - \\
\hline 3197 & $t>c$ & $\mathrm{np}$ & No & - & - & MT-RNR2 / 16S ribosomal RNA & 2 & $7.1 \%$ & $3.3 \%$ \\
\hline 3212 & $c>t$ & $\mathrm{np}$ & No & - & - & MT-RNR2 / 16S ribosomal RNA & 1 & $3.6 \%$ & $0.2 \%$ \\
\hline 3394 & $t>c$ & $\mathrm{np}$ & No & - & $Y>H$ & MT-ND1 & 2 & $7.1 \%$ & $1.6 \%$ \\
\hline 3450 & $c>t$ & $\mathrm{np}$ & No & - & Syn & MT-ND1 & 1 & $3.6 \%$ & $0.7 \%$ \\
\hline 3480 & $a>g$ & $\mathrm{np}$ & No & - & Syn & MT-ND1 & 3 & $10.7 \%$ & $4.1 \%$ \\
\hline 3594 & $c>t$ & $\mathrm{np}$ & No & - & Syn & MT-ND1 & 2 & $7.1 \%$ & $6.0 \%$ \\
\hline 4104 & $a>g$ & $\mathrm{np}$ & No & - & Syn & MT-ND1 & 2 & $7.1 \%$ & $6.0 \%$ \\
\hline 4216 & $t>c$ & $t>c$ & No & - & $Y>H$ & MT-ND1 & 6 & $21.4 \%$ & $9.5 \%$ \\
\hline
\end{tabular}




\begin{tabular}{|c|c|c|c|c|c|c|c|c|c|}
\hline 4580 & $g>a$ & $\mathrm{np}$ & No & - & Syn & MT-ND2 & 1 & $3.6 \%$ & $2.9 \%$ \\
\hline 4732 & $a>g$ & $\mathrm{np}$ & No & - & $N>S$ & MT-ND2 & 1 & $3.6 \%$ & $1.2 \%$ \\
\hline 4769 & $a>g$ & $\mathrm{np}$ & No & - & Syn & MT-ND2 & 27 & $96.4 \%$ & $98.9 \%$ \\
\hline 4917 & $a>g$ & $a>g$ & No & - & $N>D$ & MT-ND2 & 3 & $10.7 \%$ & $4.9 \%$ \\
\hline 5046 & $g>a$ & $g>a$ & No & - & $V>1$ & MT-ND2 & 2 & $7.1 \%$ & $3.2 \%$ \\
\hline 5108 & $t>c$ & $\mathrm{np}$ & No & - & Syn & MT-ND2 & 1 & $3.6 \%$ & $3.1 \%$ \\
\hline 5147 & $g>a$ & np & No & - & Syn & MT-ND2 & 1 & $3.6 \%$ & $5.3 \%$ \\
\hline 5471 & $g>a$ & $\mathrm{np}$ & No & - & Syn & MT-ND2 & 1 & $3.6 \%$ & $0.7 \%$ \\
\hline 5655 & $t>c$ & $t>c$ & No & - & - & MT-TA & 2 & $7.1 \%$ & $0.9 \%$ \\
\hline 5773 & $g>a$ & $g>a$ & No & - & - & MT-TC & 1 & $3.6 \%$ & $2.2 \%$ \\
\hline 5913 & $g>a$ & $n p$ & No & - & $D>N$ & MT-CO1 & 1 & $3.6 \%$ & $0.4 \%$ \\
\hline 6018 & $g>a$ & np & No & - & $A>T$ & MT-C01 & 1 & $3.6 \%$ & $0.1 \%$ \\
\hline 6221 & $t>c$ & $\mathrm{np}$ & No & - & Syn & MT-C01 & 1 & $3.6 \%$ & $2.3 \%$ \\
\hline 6261 & $g>a$ & $g>a$ & No & - & $A>T$ & MT-C01 & 1 & $3.6 \%$ & $0.4 \%$ \\
\hline 6776 & $t>c$ & $\mathrm{np}$ & No & - & Syn & MT-C01 & 2 & $7.1 \%$ & $2.1 \%$ \\
\hline 6962 & $g>a$ & $\mathrm{np}$ & No & - & Syn & MT-C01 & 1 & $3.6 \%$ & $1.9 \%$ \\
\hline 7028 & $c>t$ & np & No & - & Syn & MT-C01 & 15 & $53.6 \%$ & $81.5 \%$ \\
\hline 7055 & $a>g$ & $\mathrm{np}$ & No & - & Syn & MT-C01 & 2 & $7.1 \%$ & $2.1 \%$ \\
\hline 7256 & $c>t$ & np & No & - & Syn & MT-C01 & 2 & $7.1 \%$ & $6.0 \%$ \\
\hline 7337 & $g>a$ & $\mathrm{np}$ & No & - & Syn & MT-C01 & 1 & $3.6 \%$ & $0.6 \%$ \\
\hline 7521 & $g>a$ & $\mathrm{np}$ & No & - & - & MT-TD & 2 & $7.1 \%$ & $6.1 \%$ \\
\hline 7768 & $a>g$ & $\mathrm{np}$ & No & - & Syn & MT-CO2 & 2 & $7.1 \%$ & $1.9 \%$ \\
\hline 7867 & $c>t$ & $\mathrm{np}$ & No & - & Syn & MT-CO2 & 2 & $7.1 \%$ & $1.9 \%$ \\
\hline 8468 & $c>t$ & $\mathrm{np}$ & No & - & Syn & MT-ATP8 & 2 & $7.1 \%$ & $3.5 \%$ \\
\hline 8697 & $g>a$ & $\mathrm{np}$ & No & - & Syn & MT-ATP6 & 2 & $7.1 \%$ & $4.9 \%$ \\
\hline 8701 & $a>g$ & $\mathrm{np}$ & No & - & $T>A$ & MT-ATP6 & 3 & $10.7 \%$ & $35.1 \%$ \\
\hline 8736 & $t>c$ & $\mathrm{np}$ & No & - & Syn & MT-ATP6 & 1 & $3.6 \%$ & - \\
\hline 8854 & $g>a$ & $\mathrm{np}$ & Yes & 1 & $A>T$ & MT-ATP6 & 1 & $3.6 \%$ & $0.1 \%$ \\
\hline 8860 & $a>g$ & $\mathrm{np}$ & No & - & $T>A$ & MT-ATP6 & 27 & $96.4 \%$ & $99.8 \%$ \\
\hline 8901 & $a>g$ & $\mathrm{np}$ & No & - & Syn & MT-ATP6 & 1 & $3.6 \%$ & $0.1 \%$ \\
\hline 9055 & $g>a$ & np & No & - & $A>T$ & MT- ATP6 & 3 & $10.7 \%$ & $4.2 \%$ \\
\hline 9449 & $c>t$ & $\mathrm{np}$ & No & - & Syn & MT-CO3 & 1 & $3.6 \%$ & $1.1 \%$ \\
\hline
\end{tabular}




$\begin{array}{lll}9477 & \mathrm{~g}>\mathrm{a} & \mathrm{np} \\ 9670 & \mathrm{a}>\mathrm{g} & \mathrm{np} \\ 9698 & \mathrm{t}>\mathrm{c} & \mathrm{np} \\ 9899 & \mathrm{t}>\mathrm{c} & \mathrm{np} \\ 10086 & \mathrm{a}>\mathrm{g} & \mathrm{np} \\ 10172 & \mathrm{~g}>\mathrm{a} & \mathrm{np} \\ 10325 & \mathrm{~g}>\mathrm{a} & \mathrm{np} \\ 10373 & \mathrm{~g}>\mathrm{a} & \mathrm{np} \\ 10398 & \mathrm{a}>\mathrm{g} & \mathrm{np} \\ 10463 & \mathrm{t}>\mathrm{c} & \mathrm{np} \\ 10550 & \mathrm{a}>\mathrm{g} & \mathrm{np} \\ 10750 & \mathrm{a}>\mathrm{g} & \mathrm{np} \\ 10873 & \mathrm{t>c} & \mathrm{np} \\ 11002 & \mathrm{a}>\mathrm{g} & \mathrm{np} \\ 11251 & \mathrm{a}>\mathrm{g} & \mathrm{np} \\ 11299 & \mathrm{t}>\mathrm{c} & \mathrm{np} \\ 11467 & \mathrm{a}>\mathrm{g} & \mathrm{np} \\ 11653 & \mathrm{a}>\mathrm{g} & \mathrm{np} \\ 11719 & \mathrm{~g}>\mathrm{a} & \mathrm{np} \\ 11812 & \mathrm{a}>\mathrm{g} & \mathrm{np} \\ 12338 & \mathrm{t}>\mathrm{c} & \mathrm{np} \\ 12372 & \mathrm{~g}>\mathrm{a} & \mathrm{np} \\ 12612 & \mathrm{a}>\mathrm{g} & \mathrm{np} \\ 12634 & \mathrm{a}>\mathrm{g} & \mathrm{np} \\ 12705 & \mathrm{c}>\mathrm{t} & \mathrm{np} \\ 12717 & \mathrm{c}>\mathrm{t} & \mathrm{np} \\ 12738 & \mathrm{t}>\mathrm{g} & \mathrm{np} \\ 13105 & \mathrm{a}>\mathrm{g} & \mathrm{np} \\ 13326 & \mathrm{t}>\mathrm{c} & \mathrm{np} \\ 13327 & \mathrm{a}>\mathrm{g} & \mathrm{np} \\ 13368 & \mathrm{~g}>\mathrm{a} & \mathrm{np} \\ 13617 & \mathrm{t}>\mathrm{c} & \mathrm{np}\end{array}$

\begin{tabular}{|c|c|c|c|}
\hline No & - & $V>1$ & MT-CO3 \\
\hline No & - & $N>S$ & MT-CO3 \\
\hline No & - & Syn & MT-CO3 \\
\hline No & - & Syn & MT-CO3 \\
\hline No & - & $\mathrm{N}>\mathrm{D}$ & MT-ND3 \\
\hline No & - & Syn & MT-ND3 \\
\hline No & - & Syn & MT-ND3 \\
\hline No & - & Syn & MT-ND3 \\
\hline No & - & $T>A$ & MT-ND3 \\
\hline No & - & - & MT-TR \\
\hline No & - & Syn & MT-ND4L \\
\hline No & - & $N>S$ & MT-ND4L \\
\hline No & - & Syn & MT-ND4 \\
\hline No & - & Syn & MT-ND4 \\
\hline No & - & Syn & MT-ND4 \\
\hline No & - & Syn & MT-ND4 \\
\hline No & - & Syn & MT-ND4 \\
\hline No & - & Syn & MT-ND4 \\
\hline No & - & Syn & MT-ND4 \\
\hline No & - & Syn & MT-ND4 \\
\hline No & - & $M>T$ & MT-ND5 \\
\hline No & - & Syn & MT-ND5 \\
\hline No & - & Syn & MT-ND5 \\
\hline No & - & $1>V$ & MT-ND5 \\
\hline No & - & Syn & MT-ND5 \\
\hline No & - & Syn & MT-ND5 \\
\hline No & - & Syn & MT-ND5 \\
\hline No & - & $1>V$ & MT-ND5 \\
\hline No & - & Syn & MT-ND5 \\
\hline No & - & $\mathrm{T}>\mathrm{A}$ & MT-ND5 \\
\hline No & - & Syn & MT-ND5 \\
\hline No & - & Syn & MT-ND5 \\
\hline
\end{tabular}

\begin{tabular}{ll}
$7.1 \%$ & $3.4 \%$ \\
$3.6 \%$ & $0.2 \%$ \\
$10.7 \%$ & $4.4 \%$ \\
$7.1 \%$ & $1.1 \%$ \\
$3.6 \%$ & $1.1 \%$ \\
$3.6 \%$ & $0.4 \%$ \\
$3.6 \%$ & $0.5 \%$ \\
$3.6 \%$ & $1.1 \%$ \\
$28.6 \%$ & $46.2 \%$ \\
$10.7 \%$ & $4.9 \%$ \\
$10.7 \%$ & $3.6 \%$ \\
$3.6 \%$ & $0.2 \%$ \\
$10.7 \%$ & $35.5 \%$ \\
$3.6 \%$ & $0.6 \%$ \\
$17.9 \%$ & $9.1 \%$ \\
$14.3 \%$ & $4.3 \%$ \\
$17.9 \%$ & $11.0 \%$ \\
$3.6 \%$ & $0.2 \%$ \\
$46.4 \%$ & $77.8 \%$ \\
$3.6 \%$ & $3.3 \%$ \\
$3.6 \%$ & $0.2 \%$ \\
$17.9 \%$ & $12.8 \%$ \\
$7.1 \%$ & $4.4 \%$ \\
$3.6 \%$ & $0.1 \%$ \\
$10.7 \%$ & $46.6 \%$ \\
$3.6 \%$ & - \\
$3.6 \%$ & $0.2 \%$ \\
$14.3 \%$ & $6.2 \%$ \\
$3.6 \%$ & $0.3 \%$ \\
$3.6 \%$ & - \\
$10.7 \%$ & $5.1 \%$ \\
$7.1 \%$ & $3.3 \%$ \\
\hline
\end{tabular}




\begin{tabular}{|c|c|c|c|c|c|c|c|c|c|}
\hline 13637 & $a>g$ & $\mathrm{np}$ & No & - & $Q>R$ & MT-ND5 & 2 & $7.1 \%$ & $0.5 \%$ \\
\hline 13650 & $c>t$ & $\mathrm{np}$ & No & - & Syn & MT-ND5 & 2 & $7.1 \%$ & $6.0 \%$ \\
\hline 13680 & $c>t$ & $\mathrm{np}$ & No & - & Syn & MT-ND5 & 1 & $3.6 \%$ & $0.6 \%$ \\
\hline 13708 & $g>a$ & $\mathrm{np}$ & No & - & $A>T$ & MT-ND5 & 2 & $7.1 \%$ & $6.8 \%$ \\
\hline 13789 & $t>c$ & $\mathrm{np}$ & No & - & $Y>H$ & MT-ND5 & 2 & $7.1 \%$ & $0.1 \%$ \\
\hline 14178 & $t>c$ & $\mathrm{np}$ & No & - & $1>V$ & MT-ND6 & 2 & $7.1 \%$ & $2.2 \%$ \\
\hline 14182 & $t>c$ & $\mathrm{np}$ & No & - & Syn & MT-ND6 & 2 & $7.1 \%$ & $2.3 \%$ \\
\hline 14233 & $a>g$ & $\mathrm{np}$ & No & - & Syn & MT-ND6 & 1 & $3.6 \%$ & $3.4 \%$ \\
\hline 14470 & $t>a$ & $\mathrm{np}$ & No & - & Syn & MT-ND6 & 1 & $3.6 \%$ & $0.3 \%$ \\
\hline 14560 & $g>a$ & $n p$ & No & - & Syn & MT-ND6 & 2 & $7.1 \%$ & $2.3 \%$ \\
\hline 14769 & $a>g$ & $a>g$ & No & - & $N>S$ & MT-CYB & 2 & $7.1 \%$ & $1.0 \%$ \\
\hline 14798 & $t>c$ & $\mathrm{np}$ & No & - & $\mathrm{F}>\mathrm{L}$ & MT-CYB & 5 & $17.9 \%$ & $7.5 \%$ \\
\hline 14872 & $c>t$ & $\mathrm{np}$ & No & - & Syn & MT-CYB & 1 & $3.6 \%$ & $0.4 \%$ \\
\hline 14905 & $g>a$ & $\mathrm{np}$ & No & - & Syn & MT-CYB & 3 & $10.7 \%$ & $5.3 \%$ \\
\hline 15115 & $t>c$ & $\mathrm{np}$ & No & - & Syn & MT-CYB & 2 & $7.1 \%$ & $1.2 \%$ \\
\hline 15217 & $g>a$ & $\mathrm{np}$ & No & - & Syn & MT-CYB & 1 & $3.6 \%$ & $1.2 \%$ \\
\hline 15301 & $g>a$ & $\mathrm{np}$ & Yes & 1 & Syn & MT-CYB & 2 & $7.1 \%$ & $32.4 \%$ \\
\hline 15326 & $a>g$ & $\mathrm{np}$ & No & - & $\mathrm{T}>\mathrm{A}$ & MT-CYB & 27 & $96.4 \%$ & $99.3 \%$ \\
\hline 15452 & $c>a$ & $c>a$ & Yes & 1 & $L>1$ & MT-CYB & 5 & $17.9 \%$ & $9.1 \%$ \\
\hline 15607 & $a>g$ & $\mathrm{np}$ & No & - & Syn & MT-CYB & 3 & $10.7 \%$ & $5.7 \%$ \\
\hline 15833 & $c>t$ & $\mathrm{np}$ & No & - & Syn & MT-CYB & 1 & $3.6 \%$ & $0.9 \%$ \\
\hline 15904 & $c>t$ & $\mathrm{np}$ & No & - & - & MT-TT & 1 & $3.6 \%$ & $2.8 \%$ \\
\hline 15928 & $g>a$ & $\mathrm{np}$ & No & - & - & MT-TT & 3 & $10.7 \%$ & $5.0 \%$ \\
\hline \multicolumn{10}{|c|}{ polymorphisms - synchronous variations } \\
\hline Position & $\begin{array}{l}\text { nt-change } \\
\text { Chip }\end{array}$ & $\begin{array}{l}\text { nt-change } \\
\text { custom sequencing }\end{array}$ & $\begin{array}{l}\text { Heteroplasmic } \\
\text { on chip }\end{array}$ & $\begin{array}{l}\text { In No. of samples } \\
\text { heteroplasmic on chip }\end{array}$ & aa-change & Locus & No. of samples & $\%$ of samples & Population frequency * \\
\hline 3579 & $a>g$ & $\mathrm{np}$ & No & - & Syn & MT-ND1 & 1 & $3.6 \%$ & - \\
\hline 3666 & $g>a$ & $\mathrm{np}$ & No & - & Syn & MT-ND1 & 2 & $7.1 \%$ & $2.2 \%$ \\
\hline 3693 & $g>a$ & $\mathrm{np}$ & No & - & Syn & MT-ND1 & 2 & $7.1 \%$ & $1.1 \%$ \\
\hline 4562 & $a>g$ & $\mathrm{np}$ & No & - & Syn & MT-ND2 & 1 & $3.6 \%$ & $0.4 \%$ \\
\hline
\end{tabular}




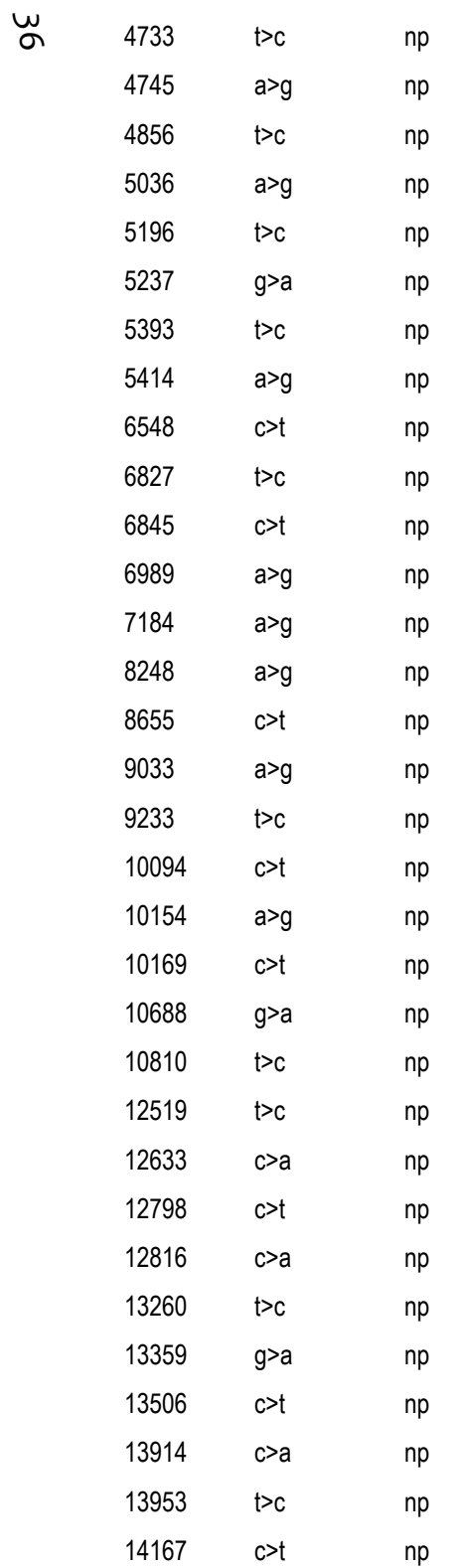

\begin{tabular}{|c|c|c|c|}
\hline No & - & Syn & MT-ND2 \\
\hline No & - & Syn & MT-ND2 \\
\hline No & - & Syn & MT-ND2 \\
\hline No & - & Syn & MT-ND2 \\
\hline No & - & Syn & MT-ND2 \\
\hline No & - & Syn & MT-ND2 \\
\hline No & - & Syn & MT-ND2 \\
\hline No & - & Syn & MT-ND2 \\
\hline No & - & Syn & MT-C01 \\
\hline No & - & Syn & MT-C01 \\
\hline No & - & Syn & MT-CO1 \\
\hline No & - & Syn & MT-C01 \\
\hline No & - & Syn & MT-C01 \\
\hline No & - & Syn & MT-CO2 \\
\hline No & - & Syn & MT-ATP6 \\
\hline No & - & Syn & MT-ATP6 \\
\hline No & - & Syn & MT-CO3 \\
\hline No & - & Syn & MT-ND3 \\
\hline No & - & Syn & MT-ND3 \\
\hline No & - & Syn & MT-ND3 \\
\hline No & - & Syn & MT-ND4L \\
\hline No & - & Syn & MT-ND4 \\
\hline No & - & Syn & MT-ND5 \\
\hline No & - & Syn & MT-ND5 \\
\hline No & - & Syn & MT-ND5 \\
\hline Yes & 1 & Syn & MT-ND5 \\
\hline No & - & Syn & MT-ND5 \\
\hline No & - & Syn & MT-ND5 \\
\hline No & - & Syn & MT-ND5 \\
\hline No & - & Syn & MT-ND5 \\
\hline No & - & Syn & MT-ND5 \\
\hline No & - & Syn & MT-ND6 \\
\hline
\end{tabular}

\begin{tabular}{ll}
$3.6 \%$ & $0.2 \%$ \\
$3.6 \%$ & $0.4 \%$ \\
$3.6 \%$ & $0.0 \%$ \\
$7.1 \%$ & $0.9 \%$ \\
$3.6 \%$ & $0.1 \%$ \\
$3.6 \%$ & $0.9 \%$ \\
$7.1 \%$ & $0.8 \%$ \\
$3.6 \%$ & $0.1 \%$ \\
$7.1 \%$ & $0.8 \%$ \\
$7.1 \%$ & $1.1 \%$ \\
$3.6 \%$ & $0.1 \%$ \\
$7.1 \%$ & $0.8 \%$ \\
$3.6 \%$ & $0.2 \%$ \\
$7.1 \%$ & $0.9 \%$ \\
$7.1 \%$ & $3.5 \%$ \\
$3.6 \%$ & $0.1 \%$ \\
$3.6 \%$ & $0.1 \%$ \\
$3.6 \%$ & - \\
$3.6 \%$ & $0.1 \%$ \\
$3.6 \%$ & $0.0 \%$ \\
$7.1 \%$ & $3.6 \%$ \\
$7.1 \%$ & $3.8 \%$ \\
$7.1 \%$ & $1.0 \%$ \\
$7.1 \%$ & $1.5 \%$ \\
$3.6 \%$ & $0.0 \%$ \\
$3.6 \%$ & - \\
$3.6 \%$ & $0.1 \%$ \\
$3.6 \%$ & $0.3 \%$ \\
$7.1 \%$ & $3.5 \%$ \\
$3.6 \%$ & $0.7 \%$ \\
$3.6 \%$ & - \\
$10.7 \%$ & $4.2 \%$ \\
\hline &
\end{tabular}




\begin{tabular}{|c|c|c|c|c|c|c|c|c|c|}
\hline 14203 & $a>g$ & $n p$ & No & - & Syn & MT-ND6 & 2 & $7.1 \%$ & $0.9 \%$ \\
\hline 14773 & $c>t$ & $\mathrm{np}$ & No & - & Syn & MT-CYB & 1 & $3.6 \%$ & - \\
\hline 14956 & $t>c$ & $\mathrm{np}$ & No & - & Syn & MT-CYB & 1 & $3.6 \%$ & $0.0 \%$ \\
\hline 15340 & $a>g$ & $\mathrm{np}$ & Yes & 1 & Syn & MT-CYB & 1 & $3.6 \%$ & $0.1 \%$ \\
\hline 15808 & $a>g$ & $\mathrm{np}$ & No & - & Syn & MT-CYB & 1 & $3.6 \%$ & $0.1 \%$ \\
\hline
\end{tabular}

* Frequencies in the general population were retrieved from the Human Mitochondrial Genome Database were sequence information is available from 2469 mtDNA sequences (June 28, 2006) [61]. np: Not performed.

Syn: synchronous variation (no change in amino acid) 



\section{CHAPTER 3}

\section{Gene expression profiling reveals complement mediated regeneration in skeletal muscle from Leigh syndrome patients with a SURF1 mutation}

R.G.E. van Eijsden ${ }^{1,2, \$}$, R. Mineri ${ }^{3, \$}$, P.J. Lindsey ${ }^{1,2}$, L.M.T. Eijssen ${ }^{1}$, C.M.M. van den Burg ${ }^{1,2}$, L.E.A. de Wit $^{4}$, T.A. Ayoubi ${ }^{1}$, C. Di Blasi ${ }^{5}$, J. Zeman ${ }^{6}$, M. Zeviani ${ }^{3}$, I.F.M. de Coo ${ }^{7}$, W. Sluiter ${ }^{4}$, H.J.M. Smeets 1,2,E, V. Tiranti ${ }^{3, \mathrm{E}}$

'Department of Genetics and Cell Biology, Maastricht University, Maastricht - The Netherlands. ${ }^{2}$ Research Institute Growth \& Development, Maastricht University, Maastricht - The Netherlands. ${ }^{3}$ Pierfranco and Luisa Mariani Centre for the Study of Children's Mitochondrial Disorders, IRCCS Foundation Neurological Institute "C. Besta", Unit of Molecular Neurogenetics, Milan - Italy. ${ }^{4}$ Department of Biochemistry, Mitochondrial Research Unit, Erasmus MC, Rotterdam - The Netherlands.

${ }^{5}$ Unit of Neuromuscular diseases and Neuroimmunology, IRCCS Foundation Neurological Institute "C. Besta",

${ }^{6}$ Department of Paediatrics, First Faculty of Medicine, Charles University, Prague, Czech Republic ${ }^{7}$ Department of Neurology, Erasmus MC, Rotterdam - The Netherlands.

\$Joint first authors.

${ }^{\text {f}}$ Both senior authors contributed equally to this work.

Submitted 


\begin{abstract}
Leigh syndrome is an early-onset and often fatal neurodegenerative disorder, characterised by necrotic lesions in the brain basal ganglia. Leigh syndrome with a decreased cytochrome c oxidase (COX) activity is frequently caused by SURF1 gene mutations. To characterise molecular pathophysiological processes, gene expression profiling was performed in skeletal muscle biopsies from SURF1 Leigh syndrome patients and controls. No significant alterations were observed for the oxidative phosphorylation (OXPHOS) genes. Altered were protein synthesis, DNA metabolism, cell cycle, skeletal muscle development, and intriguingly the complement system. Genes of the classical complement pathway ( $C_{1} R, C_{1} S$, and $\left.C_{3}\right)$, involved in both immune response and tissue regeneration, were significantly up-regulated. This regenerative response is also observed by other processes. Most likely, SURF1 mutations lead to increased production of reactive oxygen species (ROS), causing protein damage and increased turnover. This could trigger complement mediated muscle regeneration as a rescue process.
\end{abstract}

\title{
Introduction
}

Leigh syndrome (MIM 256000) is a progressive neurodegenerative disorder of infancy and childhood. The onset of the disease is at birth or within the first months of life. It has a characteristic neuropathology of focal, bilateral symmetrical necrotic lesions in brainstem, basal ganglia, thalamus and spinal cord. Disease progression is in general very fast, resulting in death within about two years from the age of onset. A frequent cause of Leigh syndrome is a defective oxidative phosphorylation (OXPHOS) system [62]. Mutations have been identified mainly in genes coding for enzymes involved in aerobic energy metabolism, including the X-linked E1 $\alpha$ subunit of pyruvate dehydrogenase, the subunits of the OXPHOS system, and genes involved in the assembly of these complexes. Some of these genes are encoded by the nuclear DNA, others by the mitochondrial DNA (mtDNA). Mutations in the SURF1 gene, which is encoded by the nuclear DNA, are the main cause of Leigh syndrome with cytochrome c oxidase (COX) deficiency. Tiranti et al. reported $75 \%$ (18 out of 24 ) of the Leigh patients with COX deficiency to have a mutation in the SURF1 gene [63], although another study reported a lower frequency of $26 \%$ ( 6 out of 23) [64]. The absence of the SURF1 protein causes accumulation of early assembly intermediates ( $\mathrm{S} 1$ and S2) of the cytochrome c oxidase complex, as is illustrated by blue native two-dimensional gel electrophoresis, indicating that the SURF1 protein plays a role in the assembly of intermediate component S3 [65]. Leigh syndrome caused by mutations in the SURF1 gene has a homogeneous clinical manifestation. Patients present with progressive encephalopathy, generalised hypotonia with sharp tendon reflexes, trunk ataxia, oculomotor abnormalities, central respiratory problems, and rapidly progressive psychomotor regression [7]. Although we know that Surf1 is an important factor for the assembly of the COX complex, the exact pathophysiological mechanism in the 
development and progression of the disease is still unknown and it is also unclear whether the SURF1 protein has additional roles in other biological processes [32].

Mitochondrial diseases mainly affect tissues with a high energy demand like heart, skeletal muscle, and the renal and neuro-endocrine systems [7]. In Leigh syndrome these are the central nervous system and skeletal muscle [7]. To identify pathogenic molecular processes due to mutations in the SURF1 gene, and to gain more insight into the pathophysiology of the disease, differences in skeletal muscle gene expression levels between SURF1 patients and controls were characterised by microarray technology and validated by functional assays.

\section{Materials \& methods}

Patients and controls

Needle biopsies were taken from the quadriceps muscle from six Leigh syndrome patients with a mutation in the SURF1 gene (P60, P62, P63, P64, P65, P66) and from six controls (C3-8, table 1). For ethical reasons it was not possible to systematically get age-matched controls, which would involve healthy children of 2-4 years of age, and only one biopsy was available from a 10-year old girl with no mitochondrial disease, which was used for immunohistochemistry (see below). Three of the Leigh syndrome patients have been previously described by Tiranti et al. (patient 6 in table 1, patient P64 in table 1 of this study), Bruno et al. (patient P63 in table 1 of this study), and Zhu et al. (patient P6o in table 1 of this study) [8, 63, 66]. After biopsy, the muscle samples were immediately frozen in liquid nitrogen.

\section{Microarray procedure}

Total RNA was isolated using the TRIzol reagent (Invitrogen, Breda, The Netherlands) and purified with the RNeasy clean-up kit (Qiagen, Venlo, The Netherlands). RNA quantity and purity were determined spectrophotometrically using the Nanodrop ND-1000 (Nanodrop Technologies, Wilmington, DE, USA) and RNA integrity was assessed by determining the RNA 28S/18S ratio using a Bioanalyser 2100 (Agilent, Santa Clara , CA, USA). Muscle RNA spiked with four bacterial RNA transcripts was reverse transcribed into CDNA and amplified in a two-round amplification reaction according to the manufacturer's protocol (Affymetrix, Santa Clara, CA, USA). A mixture of cDNA and added hybridisation controls was hybridised on Affymetrix HG-U95Av2 chips, followed by staining and washing steps in the GeneChip fluidics station according to the manufacturer's procedures. To assess the raw probe signal intensities, chips were scanned using the GeneChip scanner 3000 (Affymetrix, Santa Clara, CA, USA). 
Table 1: Patients and controls

\begin{tabular}{|c|c|c|c|c|c|}
\hline \multirow{2}{*}{$\begin{array}{l}\text { Subject- } \\
\text { Code }\end{array}$} & \multirow[t]{2}{*}{ SURF1 Mutations } & \multirow[t]{2}{*}{ Sex } & \multirow[t]{2}{*}{ Age at muscle biopsy } & \multirow[b]{2}{*}{ COX activity } & \multirow[b]{2}{*}{ Blood lactate leve } \\
\hline & & & & & \\
\hline P60 & [312_321del 311_312insAT] & $\bar{M}$ & 3 & $4 \%$ & increased \\
\hline & 311_312insAT] & & & & \\
\hline P62 & [19_35dup (now 37_38ins)] + [19_35dup (now 37_38ins)] & M & 4 & $21 \%$ & mildly increased \\
\hline P63 & {$[240+1 \mathrm{G}>\mathrm{T}]+\left[531 \_534 \mathrm{del}\right]$} & M & 2 & reported reduced & increased \\
\hline P64 & [19_35dup (now 37_38ins)] + [19_35dup (now 37_38ins)] & $\mathrm{F}$ & 2 & $13 \%$ & increased \\
\hline P65 & {$\left[722 \_723 i n s C\right]+\left[722 \_723 i n s C\right]$} & M & 3 & NA & NA \\
\hline P66 & {$[240+1 G>T]+[T 844 C]$} & M & 2 & $39 \%$ & increased \\
\hline P1 & [552delG] +[790-791delAG] & $\mathrm{F}$ & 10 & $15 \%$ & increased \\
\hline P2 & [312_321del 311_312insAT] + [821del18] & $\mathrm{F}$ & 2 & $5 \%$ & Increased \\
\hline C3 & - & $\mathrm{F}$ & 34 & NA & NA \\
\hline C4 & - & $\mathrm{F}$ & 64 & NA & NA \\
\hline C5 & - & $\mathrm{F}$ & 17 & NA & NA \\
\hline C6 & - & $\mathrm{F}$ & 22 & NA & NA \\
\hline $\mathrm{C7}$ & - & M & 53 & NA & NA \\
\hline C8 & - & M & 25 & NA & NA \\
\hline
\end{tabular}

Overview of patients and controls used for the microarray study and follow-up investigations.

COX activity and blood lactate levels for the controls were not determined but were assumed to be normal.

$\mathrm{P}=$ patient, $\mathrm{C}=$ control, $\mathrm{M}=$ Male $\mathrm{F}=$ Female, $\mathrm{NA}=$ Not available, $\mathrm{COX}=$ cytochrome $\mathrm{c}$ oxidase.

The nomenclature of the SURF1 mutations is as suggested by Péquignot et al. [6] according to the guidelines by Dunnen and Antonarakis [67].

\section{Microarray data analysis}

$\mathrm{R}$, a free software environment for statistical computing and graphics, was used with the gnlm and the affy libraries to perform the data preparation and analysis [40, 68-70]. Version 8 of the Unigene-based probe set definition files (CDF files) as described by Dai et al. was used to combine the individual probes into probe sets [71]. These CDF files are based on Unigene build \#192. The expresso method of the affy library, without background correction, with constant normalisation, use of perfect match signal intensities only, and the "mas" summarisation method was used to calculate the probe set intensities from the individual probe signals. Normal linear regression models, implemented by the gnlr function within the gnlm library, were used to asses significantly differentially expressed genes between patients and controls. Differences due to experimental variation were corrected for by including the data from the RNA spikes and hybridisation controls as covariates in the models. Because not all microarray experiments were carried out on the same day, the hybridisation days were also included in the modelling procedure. The Akaike information criterion (AIC), representing the likeliness of each model penalised for its complexity, was used to identify genes with a significant change in expression between the two groups [72]. The AIC was adjusted to be comparable to a two-sided chi-squared test with a significance level of $99.9 \%$, making the analysis more stringent [73]. To test for age differences we used 2 additional data sets from the Public Expression Profiling Resource (pepr.cnmcresearch.org). The first consisted of 10 controls (age $10-20$ years) and the other of 2 controls in duplo (mixed samples with age between 4 and 13 and 5 and 12 years). Because these muscle RNA samples were run on different version of 
Affymetrix CHIPs, respectively, the $133 \mathrm{~A}$ and the U95, we could only use the genes with overlapping probes or probe sets.

\section{Data mining}

To identify biological processes in which the significantly differentially expressed genes were involved, the Gene Map Annotator and Pathway Profiler (GenMAPP, version 2.1, build 20060809) and MAPPFinder (version 2.0, build 20041218) programs were used [74, 75]. The gene database version “Hs-Std_20060526.gdb" and the Mapps version "Hs_Contributed_20060605" were used for both programs. Additionally the web-based Database for Annotation, Visualisation and Integrated Discovery (DAVID) was used to assess enriched gene ontology terms within the significantly differentially expressed gene list [76].

\section{Quantitative Real-time PCR}

Primers were designed using Primer Express ${ }^{\circledR}$ software version 3.0 (Applied Biosystems, Foster City, CA, USA). CDNA was prepared from $2 \mu \mathrm{g}$ of total RNA in a standard reverse transcriptase reaction. PCR was performed in a 7700 Realtime PCR System (Applied Biosystems, Foster City, CA, USA) and a MyiQ Single-Colour Real-Time PCR Detection System (Biorad, Hercules, CA, USA) using Eurogentec qPCR $^{\mathrm{TM}}$ Mastermix Plus for SYBR Green ${ }^{\circledR}$ I (Eurogentec, Seraing, Belgium). The cycling conditions were: an initial step of 2 minutes at $50^{\circ} \mathrm{C}$, activation of the Hot Goldstar enzyme at $95^{\circ} \mathrm{C}$ for 10 minutes, and 40 cycles of 15 seconds at $95^{\circ} \mathrm{C}$ followed by 1 minute at $60^{\circ} \mathrm{C}$ (denaturation, annealing, and elongation). The ADP-ribosylation factor 1 (ARF1) gene was used as an internal reference because of its constant expression level in muscle [77]. The other 8 genes for which quantitative real-time PCR (QRT-PCR) was performed were complement component $1, r$ subcomponent ( $\left.\mathrm{C}_{1} \mathrm{R}\right)$, complement component, s subcomponent ( $\left.\mathrm{C}_{1} \mathrm{~S}\right)$, complement component 3 $(C 3)$, clusterin (CLU), pyruvate kinase, muscle (PKM2), peripheral myelin protein 22 (PMP22), ryanodine receptor 3 (RYR3), and solute carrier family 16, member 3 (SLC16A3). Primers used for amplification are listed in supplementary table S1. QRT-PCR was carried out once on the same RNA samples as used for the microarray experiments. Data was analyzed in R using the elliptic function of the growth library to implement normal linear regression models [69, 70]. The AIC was adjusted to be comparable to a two-sided chi-squared test with a significance level of $99.0 \%$, making the analysis more stringent [73].

\section{Immunohistochemistry}

For immunohistochemistry needle biopsies, taken from the quadriceps muscle patient P1 (table 1) and the age-matched control biopsy were frozen in isopentane, cooled in liquid nitrogen, and stored in liquid nitrogen until use. Immunohistochemical staining was performed on acetonefixed $6 \mu$ m-thick cryosections using anti-C3 and anti-C5b-9 monoclonal antibodies (Dako, Copenhagen, Denmark) diluted 1:10 and 1: 25, respectively. Sections were incubated in primary antibody, then in biotinylated goat anti-mouse IgG (Jackson ImmunoResearch Labs. Inc, West 
Grove, PA, USA), and finally treated with rhodamine-avidin D (Vector Labs, Burlingame, CA, USA). As a positive control for the anti- $\mathrm{C}_{3}$ staining, a cryosection of a quadriceps muscle needle biopsy from a Duchene muscular dystrophy (DMD) patient was included. For double immunolabelling, a C3C polyclonal antibody (Dako, Copenhagen, Denmark) was used in combination with an anticollagen fraction VI (membrane of muscle fibres; Chemicon, Temecula, Canada), anti-laminin a5 (subendothelial extracellular matrix; Chemicon, Temecula, Canada), and an anti-foetal or antineonatal myosin (muscle development; Novocastra, Newcastle Upon Tyne, United Kingdom) monoclonal antibody. The antibodies were diluted 1:1000, 1:250, 1:50, 1:20, and 1:20 respectively. In all cases the primary antibodies were used in the first incubation step, followed by biotinylated goat anti-rabbit IgG, by rhodamine avidin $D$ and by Cyz-labelled goat anti-mouse IgG (Jackson, West Grove, PA, USA). As a control, sections were either incubated with isotype-specific IgG or the primary antibody was omitted.

\section{Oxidative protein damage}

Oxidative damage to proteins in the muscle biopsies was determined by quantifying the amount of carbonyl groups per mg protein using an ELISA assay optimised for small amounts of proteins (Oxprot assay). For this assay, a small part of about $10 \mathrm{mg}$ wet weight ( $\mathrm{ww}$ ) of the skeletal muscle biopsies from four controls and from the same six patients used for the microarray experiments (P60, P62, P63, P64, P65, P66, table 1) was used. The snap-frozen biopsies were pulverised in the presence of $19 \mathrm{~mL} / \mathrm{g}$ ww of RIPA buffer ( $50 \mathrm{mM}$ Tris-HCl, $150 \mathrm{mM} \mathrm{NaCl}, 1 \mathrm{mM}$ EDTA, pH 7.4, containing $0.25 \%$ sodium deoxycholate, $1 \% \mathrm{NP} 40$ and supplemented with a protease inhibitor mixture (PIM) of one Complete tablet per $50 \mathrm{~mL}$ (Roche Diagnostics, Almere, The Netherlands), 1 mM 4-(2-aminoethyl)-benzenesulfonyl-fluoride hydrochloride (Pefabloc SC, Roche Diagnostics, Almere, The Netherlands) and $2 \mathrm{mM}$ diisopropyl fluorophosphate (DFP) (Fluka Chemica, Steinheim, Switzerland)) in a small Bessman tissue pulveriser (Fisher Scientific, Pittsburg, PA, USA) precooled with liquid nitrogen. For carbonyl determination the tissue homogenate was first incubated on ice for $30 \mathrm{~min}$, and then centrifuged at $16,000 \mathrm{~g}$ for $20 \mathrm{~min}$ at $4^{\circ} \mathrm{C}$, and the supernatant was diluted in PBS to $0.5 \mu \mathrm{g} / \mathrm{mL}$ protein. Next $200 \mu \mathrm{l}$ was transferred into triplicate wells of a Nunc Maxisorp microplate. The amount of carbonylgroups resulting from protein oxidation were determined as described by Alamdari et al. [78]. A technical replicate was performed for four out of eight patient samples and for four out of seven control samples. A onesided $t$-test on the averages of the replicate measurements was used to assess statistical differences between the patient and the control groups.

\section{Detection of superoxide-producing enzymes in muscle}

The expression of superoxide radical-producing enzymes in muscle was quantified by enzyme histochemistry of skeletal muscle cryosections from two SURF1 patients $\mathrm{P}_{1}$ and $\mathrm{P}_{2}$ and the 6 controls, using dihydroethidium (DHE) to trap superoxide as the enzyme product. Ten- $\mu \mathrm{m}$ thick cryosections were stored at $-80^{\circ} \mathrm{C}$ and after thawing the sections were stained by $5 \mu \mathrm{M}$ 
dihydroethidium and $0.5 \mu \mathrm{g} / \mathrm{mL}$ Hoechst 33258 in phosphate-buffered saline (PBS) for $30 \mathrm{~min}$ at $37^{\circ} \mathrm{C}$ in a humidified atmosphere [42]. The orange fluorescence of the superoxide-specific product of DHE oxidation [79] was measured using a fluorescence inverted microscope (Olympus IX50) equipped with a 460-490 $\mathrm{nm}$ band pass excitation filter and 515-nm emission IF-barrier filter, digitised with a F-view camera (Soft Imaging System, Münster, Germany), and analyzed offline (AnalySIS 3.1; Soft Imaging System, Münster, Germany). The signal was expressed in arbitrary units reflecting the total fluorescence intensity of all events divided by the number of blue fluorescent nuclei. At least 300 nuclei per section were counted in two to three consecutive sections.

\section{Results}

Gene expression profiling of muscle from SURF1 patients

Probe signal intensities specific for 7084 transcripts were obtained for each of the six SURF1 and six control samples (table 1). Normal linear regression modelling indicated 369 transcripts to be significantly differentially expressed between the SURF1 patients and the control group (supplementary table S2), 313 of which were up-regulated and 56 were down-regulated. The majority of the changes was rather small, with only $14.1 \%$ (44) of the up-regulated genes and $8.9 \%$ (5) of the down-regulated genes having fold changes of more than 2. Expression of the SURF1 transcript was significantly down-regulated with a fold change of 2.5. Other genes which were significantly more than 2.5-fold down-regulated were PKM2 (Pyruvate kinase - muscle), RYR3 (Ryanodine receptor 3), and SLC16A3 (Solute carrier family 16 - member 3). Other up-regulated genes with a fold change of more than 2 were CLU (Clusterin), C1R (Complement component 1-r subcomponent), C1S (Complement component 1-s subcomponent), and PMP22 (Peripheral myelin protein 22), with fold changes of 3.7, 3.1, 2.3, and 4.9, respectively. As 4 out of 6 controls were females and 5 out of the 6 analysed patients were males we tested whether the expression differences could be explained by sex differences between patients and controls. Reanalysis of the 369 significant genes with sex added to the model has yielded a difference for 8 genes, 2 of which only had a sex difference and 6 of which both had a difference between patients and controls and a sex difference, so we concluded that sex did not explain our results. The age difference between patients and controls could also play a role in the gene expression changes. To test this, we used 2 control data sets of the Public Expression Profiling Resource (pepr.cnmcresearch.org). The first consisted of 10 controls (age $10-20$ years) and the other of 2 controls in duplo (mixed samples with age between 4 and 13 and 5 and 12 years). Because these muscle RNA samples were run on different version of Affymetrix CHIPs, respectively, the $133 \mathrm{~A}$ and the U95, we could only use the genes with overlapping probes or probe sets. In the first data set our results were confirmed by $75 \%$ of the genes and in the second by $43 \%$. For the other genes in the second data set it was not possible to show a difference between patients and controls, probably because of the small sample size (only 2 control mixtures) and the lab variability. 
However, the initial results of the complement system were confirmed in both additional analyses.

To identify altered processes in our own patient control dataset, an additional pathway analysis was performed. MAPPFinder analysis showed that the three most significantly changed $(p<0.05)$ local pathways or gene groups were Complement Activation (classical pathway), Ribosomal Proteins, and Regulation of Actin Cytoskeleton (table 2). Because there were two maps including the complement activation pathway, this pathway is listed twice. The most significantly changed gene ontology pathways or gene groups in the biological process category can be linked to protein synthesis, DNA metabolism, cell cycle, skeletal muscle development, and the immune system with the majority of these gene expression changes pointing towards a stimulation of these processes (table 3$)$, which are the same process terms $(p<0.05)$ as determined by DAVID (supplementary table $\mathrm{S}_{3}$ ).

Table 2: MAPPFinder results for local pathways or gene groups

\begin{tabular}{llllll}
\hline MAPP Name & \# Changed & \# Measured & \# On MAPP & \% Present & P-value \\
\hline Hs_Complement_and_Coagulation_Cascades_KEGG & 7 & 39 & 63 & 61.9 & 0.013 \\
Hs_Ribosomal_Proteins & 6 & 31 & 88 & 35.2 & 0.016 \\
Hs_Regulation_of_Actin_Cytoskeleton_KEGG & 10 & 74 & 146 & 50.7 & 0.023 \\
Hs_Complement_Activation_Classical & 3 & 9 & 17 & 52.9 & 0.024 \\
\hline
\end{tabular}

MAPPFinder results listing the significantly changed $(p<0.05)$ local pathways or gene groups between Leigh syndrome patients with a SURF1 mutation and control subjects.

Table 3: MAPPFinder results for gene ontology pathways or gene groups

\begin{tabular}{|c|c|c|c|c|c|c|}
\hline GO ID & GO Name & \# Changed & \# Measured & \# in GO & $\%$ Present & P-value \\
\hline 7046 & ribosome biogenesis & 3 & 10 & 61 & 16.4 & 0.009 \\
\hline 42254 & ribosome biogenesis and assembly & 3 & 10 & 72 & 13.9 & 0.009 \\
\hline 40008 & regulation of growth & 6 & 43 & 120 & 35.8 & 0.016 \\
\hline 45445 & myoblast differentiation & 2 & 5 & 10 & 50.0 & 0.017 \\
\hline 6289 & nucleotide-excision repair & 2 & 5 & 19 & 26.3 & 0.018 \\
\hline 9719 & response to endogenous stimulus & 7 & 58 & 238 & 24.4 & 0.018 \\
\hline 1649 & osteoblast differentiation & 2 & 4 & 18 & 22.2 & 0.019 \\
\hline 45165 & cell fate commitment & 2 & 4 & 18 & 22.2 & 0.019 \\
\hline 48747 & muscle fiber development & 2 & 6 & 17 & 35.3 & 0.024 \\
\hline 48741 & skeletal muscle fiber development & 2 & 6 & 17 & 35.3 & 0.024 \\
\hline 48637 & skeletal muscle development & 2 & 6 & 17 & 35.3 & 0.024 \\
\hline 16049 & cell growth & 6 & 48 & 141 & 34.0 & 0.026 \\
\hline 8361 & regulation of cell size & 6 & 48 & 141 & 34.0 & 0.026 \\
\hline 6259 & DNA metabolism & 14 & 156 & 645 & 24.2 & 0.026 \\
\hline 1666 & response to hypoxia & 2 & 5 & 8 & 62.5 & 0.028 \\
\hline 43087 & regulation of GTPase activity & 2 & 5 & 39 & 12.8 & 0.029 \\
\hline
\end{tabular}




\begin{tabular}{|c|c|c|c|c|c|c|}
\hline 50793 & regulation of development & 4 & 24 & 63 & 38.1 & 0.03 \\
\hline 6281 & DNA repair & 6 & 51 & 202 & 25.2 & 0.032 \\
\hline 40007 & growth & 7 & 59 & 177 & 33.3 & 0.032 \\
\hline 48625 & myoblast cell fate commitment & 1 & 1 & 2 & 50.0 & 0.036 \\
\hline 7518 & myoblast cell fate determination & 1 & 1 & 2 & 50.0 & 0.036 \\
\hline 16570 & histone modification & 2 & 6 & 23 & 26.1 & 0.037 \\
\hline 16569 & covalent chromatin modification & 2 & 6 & 23 & 26.1 & 0.037 \\
\hline 48175 & hepatocyte growth factor biosynthesis & 1 & 1 & 1 & 100.0 & 0.042 \\
\hline 48176 & regulation of hepatocyte growth factor biosynthesis & 1 & 1 & 1 & 100.0 & 0.042 \\
\hline 48178 & negative regulation of hepatocyte growth factor biosynthesis & 1 & 1 & 1 & 100.0 & 0.042 \\
\hline 45663 & positive regulation of myoblast differentiation & 1 & 1 & 2 & 50.0 & 0.042 \\
\hline 45661 & regulation of myoblast differentiation & 1 & 1 & 2 & 50.0 & 0.042 \\
\hline 46881 & positive regulation of follicle-stimulating hormone secretion & 1 & 1 & 3 & 33.3 & 0.042 \\
\hline 46887 & positive regulation of hormone secretion & 1 & 1 & 3 & 33.3 & 0.042 \\
\hline 46882 & negative regulation of follicle-stimulating hormone secretion & 1 & 1 & 4 & 25.0 & 0.042 \\
\hline 46884 & follicle-stimulating hormone secretion & 1 & 1 & 4 & 25.0 & 0.042 \\
\hline 46880 & regulation of follicle-stimulating hormone secretion & 1 & 1 & 4 & 25.0 & 0.042 \\
\hline 46888 & negative regulation of hormone secretion & 1 & 1 & 5 & 20.0 & 0.042 \\
\hline 51048 & negative regulation of secretion & 1 & 1 & 7 & 14.3 & 0.042 \\
\hline 51128 & regulation of cell organization and biogenesis & 3 & 16 & 45 & 35.6 & 0.042 \\
\hline 51014 & actin filament severing & 1 & 1 & 2 & 50.0 & 0.043 \\
\hline 7346 & regulation of progression through mitotic cell cycle & 2 & 6 & 13 & 46.2 & 0.043 \\
\hline 46902 & regulation of mitochondrial membrane permeability & 1 & 1 & 1 & 100.0 & 0.044 \\
\hline 51095 & regulation of helicase activity & 1 & 1 & 1 & 100.0 & 0.044 \\
\hline 51097 & negative regulation of helicase activity & 1 & 1 & 1 & 100.0 & 0.044 \\
\hline 7569 & cell aging & 1 & 1 & 4 & 25.0 & 0.044 \\
\hline 8635 & caspase activation via cytochrome $\mathrm{c}$ & 1 & 1 & 6 & 16.7 & 0.044 \\
\hline 7568 & aging & 1 & 1 & 8 & 12.5 & 0.044 \\
\hline 9440 & cyanate catabolism & 1 & 1 & 2 & 50.0 & 0.045 \\
\hline 9439 & cyanate metabolism & 1 & 1 & 2 & 50.0 & 0.045 \\
\hline 19754 & one-carbon compound catabolism & 1 & 1 & 2 & 50.0 & 0.045 \\
\hline 7022 & chaperonin-mediated tubulin folding & 1 & 1 & 2 & 50.0 & 0.045 \\
\hline 42692 & muscle cell differentiation & 2 & 7 & 12 & 58.3 & 0.045 \\
\hline 15918 & sterol transport & 1 & 1 & 3 & 33.3 & 0.047 \\
\hline 30301 & cholesterol transport & 1 & 1 & 3 & 33.3 & 0.047 \\
\hline 42546 & cell wall biosynthesis & 1 & 1 & 1 & 100.0 & 0.048 \\
\hline 45229 & external encapsulating structure organization and biogenesis & 1 & 1 & 1 & 100.0 & 0.048 \\
\hline 9273 & cell wall biosynthesis (sensu Bacteria) & 1 & 1 & 1 & 100.0 & 0.048 \\
\hline 9252 & peptidoglycan biosynthesis & 1 & 1 & 1 & 100.0 & 0.048 \\
\hline 7047 & cell wall organization and biogenesis & 1 & 1 & 1 & 100.0 & 0.048 \\
\hline 31504 & cell wall organization and biogenesis (sensu Bacteria) & 1 & 1 & 1 & 100.0 & 0.048 \\
\hline 9214 & cyclic nucleotide catabolism & 1 & 1 & 1 & 100.0 & 0.048 \\
\hline 30202 & heparin metabolism & 1 & 1 & 2 & 50.0 & 0.048 \\
\hline 30210 & heparin biosynthesis & 1 & 1 & 2 & 50.0 & 0.048 \\
\hline 48247 & lymphocyte chemotaxis & 1 & 1 & 4 & 25.0 & 0.048 \\
\hline 15012 & heparan sulfate proteoglycan biosynthesis & 1 & 1 & 6 & 16.7 & 0.048 \\
\hline
\end{tabular}

MAPPFinder results listing the significantly changed $(p<0.05)$ gene ontology pathways or gene groups between Leigh syndrome patients with a SURF1 mutation and control subjects. 
Microarray results were validated by QRT-PCR for a number of transcripts which showed a significant 2 fold or more increase or decrease according to the microarray data analysis ( $C_{1} R, C_{1} S$, CLU, PKM2, PMP22, RYR3, SLC16A3 - supplementary table S2). To confirm the role of the complement system, we determined differences in gene transcript levels for complement component $3\left(C_{3}\right)$ as well. Transcript levels for these complement proteins were highly upregulated in the SURF1 patient samples (table 4). The expression of the transcripts coding for the pyruvate kinase muscle (PKM2), peripheral myelin protein 22 (PMP22), ryanodine receptor 3 (RYR3), and solute carrier family 16 member 3 (SLC16A3) were also significantly differentially expressed in the patient samples (table 4). QRT-PCR confirmed the results of the microarray experiment, although the fold changes were consistently larger.

Table 4: QRT-PCR validation

\begin{tabular}{|c|c|c|c|c|c|c|}
\hline & & & & & & \\
\hline & & & Array & & QRT-PCR & \\
\hline Symbol & Name & Process & Significant & FC & Significant & FC \\
\hline$\overline{C 1 R}$ & $\begin{array}{l}\text { Complement component } 1 \text {, } \\
\text { r subcomponent }\end{array}$ & classical pathway & $\begin{array}{l}\text { Yes } \\
\end{array}$ & $3.1 \uparrow$ & $\begin{array}{l}\text { Yes } \\
\end{array}$ & $9.2 \uparrow$ \\
\hline C1S & $\begin{array}{l}\text { Complement component } 1 \text {, } \\
\text { s subcomponent }\end{array}$ & classical pathway & Yes & $2.3 \uparrow$ & Yes & $10.5 \uparrow$ \\
\hline C3 & Complement component 3 & classical/alternative pathway & - & - & Yes & $9.7 \uparrow$ \\
\hline CLU & Clusterin & Complement & Yes & $3.7 \uparrow$ & Yes & $9.1 \uparrow$ \\
\hline PKM2 & Pyruvate kinase, muscle & Energy metabolism & Yes & $2.5 \downarrow$ & Yes & $5.0 \downarrow$ \\
\hline PMP22 & Peripheral myelin protein 22 & Myelinisation & Yes & $4.9 \uparrow$ & Yes & $10.0 \uparrow$ \\
\hline RYR3 & Ryanodine receptor 3 & Calcium metabolism & Yes & $2.5 \downarrow$ & Yes & $3.3 \downarrow$ \\
\hline SLC16A3 & $\begin{array}{l}\text { Solute carrier family } 16 \\
\text { (monocarboxylic acid transporters), } \\
\text { member } 3\end{array}$ & Lactate transport & Yes & $2.5 \downarrow$ & Yes & $5.0 \downarrow$ \\
\hline
\end{tabular}

Gene transcript fold changes for SURF1 Leigh patients compared to controls determined by microarray analysis (Array) and quantitative realtime pcr (QRT-PCR) with addition of complement component $C_{3}$ (not on the array). FC: fold change, $\uparrow$ : up-regulation, $\downarrow$ : down-regulation.

Immunohistochemistry of the complement system in muscle of a SURF1 patient

Immunohistochemical staining of complement component $\mathrm{C}_{3}$ was much stronger in muscle of an SURF1 patient P1 (table 1) compared to an age-matched control, in which practically no staining was observed (figure 1). Staining of $\mathrm{C}_{3}$ in muscle of a Duchene muscular dystrophy patient showed a more diffuse pattern than the SURF1 patient (figure 1, panel A). The C3 positive staining in the SURF1 patient was localised at the muscle fibre membranes, as illustrated by the colocalisation of complement component $\mathrm{C} 3 \mathrm{C}$ and collagen fraction VI (figure 1, panel B). No colocalisation in the double staining of $\mathrm{C}_{3} \mathrm{C}$ and laminin as could be identified, indicating that the complement C3C presence was confined to the muscle fibre membranes (figure 1, panel C). The double staining of $\mathrm{C}_{3} \mathrm{C}$ and anti-foetal or anti-neonatal myosin showed a few myosin positive muscle fibres in the patient samples only (figure 1, panels D and E). Immunohistochemical staining of C5b-9 complement components to identify the membrane attack complex (MAC) indicated no difference between the patient and the control (figure 1, panel F). 
Oxidative protein damage and expression of enzymes producing superoxide radicals in muscle of SURF1 patients

Oxidative damage to muscle proteins determined by the amount of carbonyl groups in the SURF1 patients was not different ( $p=0.07$ in a one-sided t-test) from that in the controls, respectively amounting to 1.98 (SD: 0.97) and 1.29 (SD: 0.74) nmol per mg protein based on 4 patients and four controls. The expression of superoxide producing enzymes was significantly increased in SURF1 muscle. As shown in figure 2, the staining intensity for these enzymes in SURF1 muscle cryosections was 2.5 fold higher than in the control samples with a p-value of $<0.005$ in a onesided t-test. 

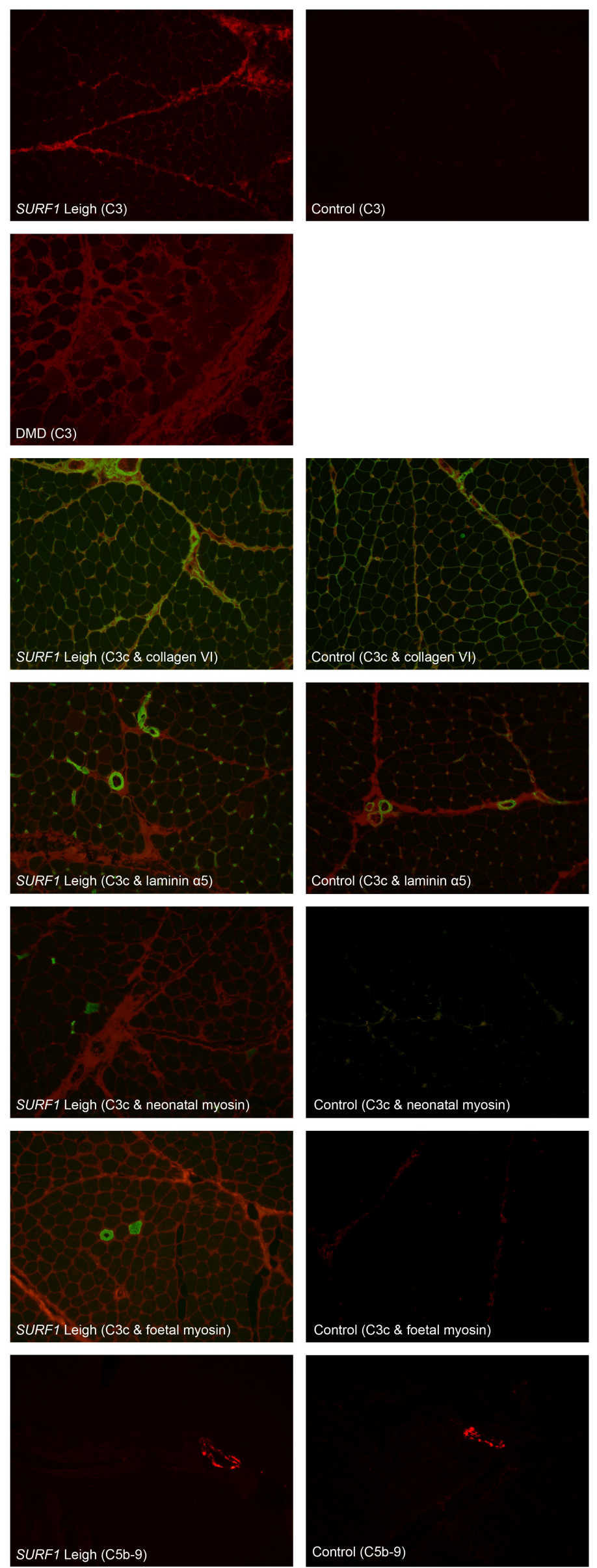
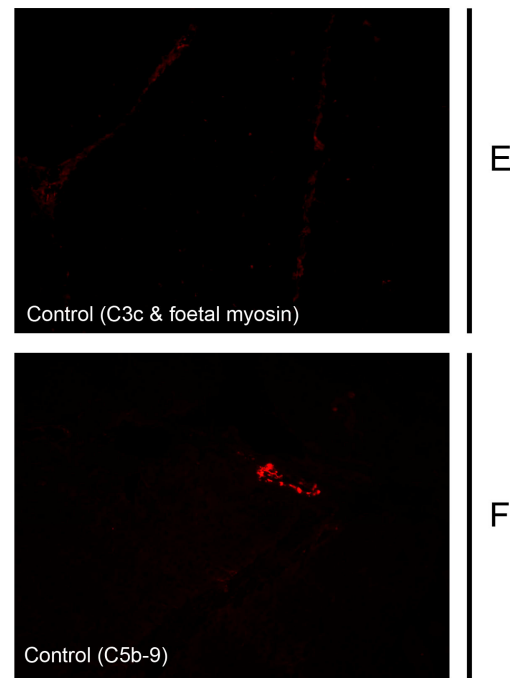

Figure 1: Immunohistochemical staining of complement factors $\mathrm{C}_{3}$ and $\mathrm{C}_{5} \mathrm{~b}-9$ in muscle of SURF1 patients

Immunohistochemical staining was performed on skeletal muscle cryosections from a control and SURF1 patient $\mathrm{P} 1$ for complement factor $\mathrm{C}_{3}$ alone (panel A), complement factor $\mathrm{C} 3 \mathrm{C}$ in combination with anti-collagen fraction VI (panel B), anti-laminin a 5 (panel C), an -neonatal (panel D) or anti-foetal (panel E) myosin monoclonal antibody and for complement component C5b-9 (panel F). Immunohistochemical staining for

B C3 was also performed on a skeletal muscle cryosection from a Duchene muscular dystrophy (DMD) patient (panel A). Staining for complement component $\mathrm{C}_{3}$ was positive for muscle of the SURF1 patient and negative for control muscle. Staining of $C_{3}$ showed a diffuse pattern for muscle of the DMD patient. C3C staining (green) of muscle of the SURF1 patient co-localised with collagen fraction $\mathrm{VI}$ (red) but not with laminin a 5 (red). A number of muscle D fibres of the SURF1 patient stained positively for both C3C (green) and anti foetal or anti neonatal myosin (red). Control muscle staining was negative for double staining against $\mathrm{C} 3 \mathrm{C}$ and anti foetal or anti neonatal myosin. Staining for C5b-9 was negative for patient P1 (panel F, left) and the control (panel $F$, right). 


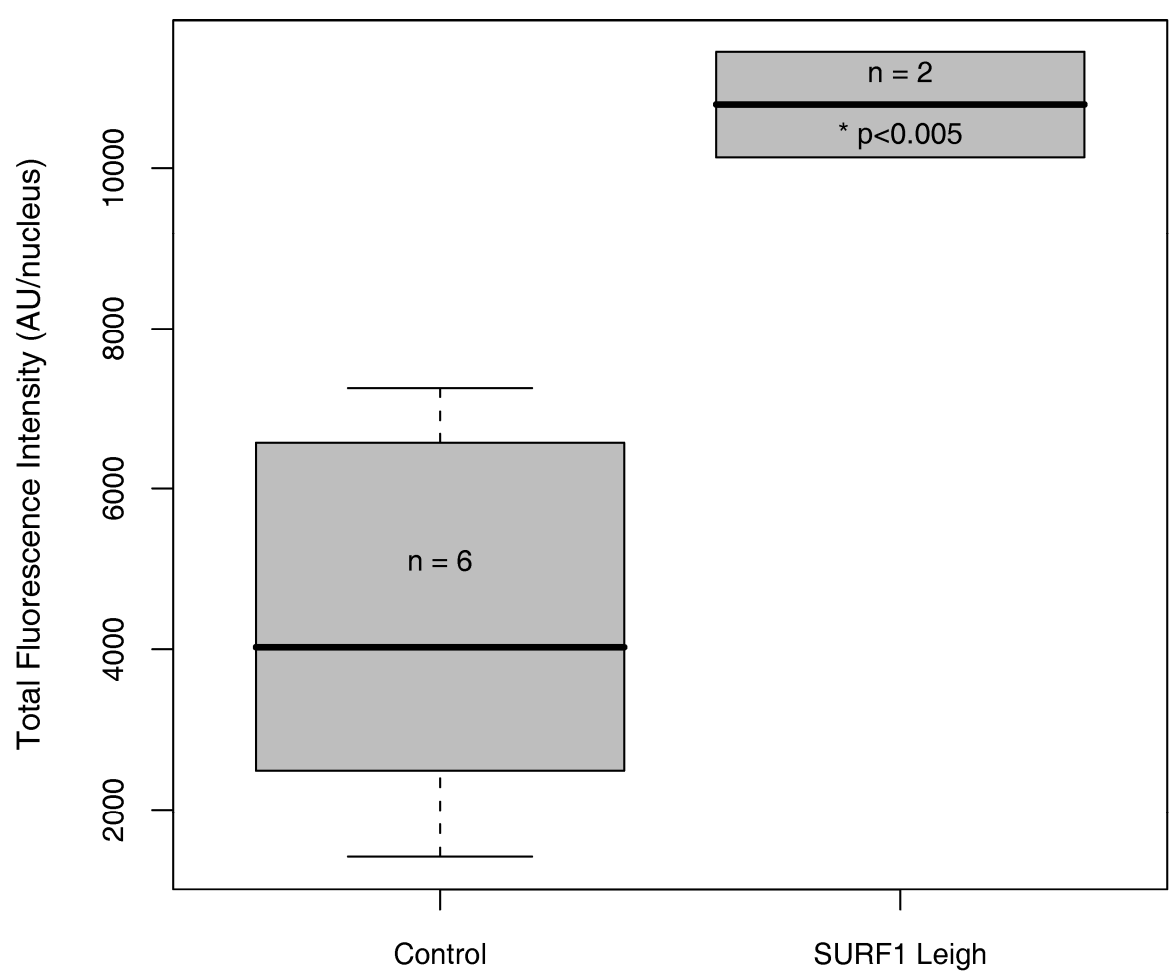

Group

\section{Figure 2: DHE staining}

Cryosections of skeletal muscle samples of controls $(n=6)$ and SURF1 patients $P 1$ and $P 2(n=2)$ were stained with dihydroethidium (DHE) to assess the amounts of superoxide radicals present. A one-sided $t$-test showed that muscle of SURF1 patients contained a 2.5 fold significantly higher amount of superoxide radicals $(p<0.005)$. The whiskers in the box plot indicate the extreme values. The boxes indicate the median (thick line) and the interquartile ranges (grey area).

\section{Discussion}

Gene expression profiling of skeletal muscle of Leigh syndrome patients with a SURF1 mutation compared to controls revealed 369 transcripts to be significantly differentially expressed (supplementary table S2). The majority (313) of the significantly differentially expressed transcripts were up-regulated, with only $14.1 \%$ of these genes having a fold change higher than 2.0. Of the 56 significantly differentially expressed transcripts which were down-regulated, $8.9 \%$ had a fold change of more than 2.0, a number of which were involved in the complement system (CLU, C1R, and C1S). The expression of 7 transcripts was confirmed by QRT-PCR (table 4). MAPPFinder and DAVID pathway and gene ontology based analyses revealed that protein synthesis, DNA metabolism, cell cycle, skeletal muscle development, and the immune system were the most significantly altered processes (tables 2, 3, and supplementary table S3). Some of 
the altered processes may not solely relate to the difference in patients and controls due to the Surf1 defect, but also to a difference in sex or age. A sex effect was excluded as an explanation for the significantly different expression of the 369 genes. An age-effect was unlikely for the majority of changes observed, although the possibility to test this properly was limited due to the data sets and the number of controls and type of CHIPs available. In addition, any control set from a different lab introduces a lab difference between patients and controls. An age-effect was unlikely to explain the differences in the complement system. It was for ethical reasons not possible to obtain systematically muscle biopsies from age-matched normal controls. The significance of the altered processes was independently validated by immunohistochemistry of a patient and the only age-matched control available and by measurement of the amount of oxidatively damaged proteins and expression of superoxide producing enzymes.

\section{Alterations in OXHPOS gene expression and energy metabolism}

The down-regulation of the SURF1 transcript (2.5 fold) was the only significant change in transcripts directly related to the OXPHOS system. This indicates that compensation for the defect in complex IV assembly does not occur by either up-regulating complex IV or other OXPHOS related genes, or genes involved in mitochondrial biogenesis in general. The significant down-regulation of PKM2 and SLC16A3 could be related to the high levels of lactate in blood and urine which are often found in Leigh syndrome patients [7]. Pyruvate kinase is responsible for the conversion of phosphoenolpyruvate to pyruvate at the end of the glycolysis reaction. If the OXPHOS system does not function properly, pyruvate will be converted to lactate resulting in higher blood and urine lactate levels. Down-regulation of the PKM2 gene coding for the pyruvate kinase enzyme might help in preventing lactic acidosis from occurring. SLC16A3, also known as monocarboxylate transporter $4(M C T 4)$ is mainly expressed in glycolytic skeletal muscle fibres and mediates the efflux of lactate from those fibres [80]. As the regulation of the monocarboxylate transporters is $\mathrm{pH}$ dependent, down-regulation of the MCT4 gene could be an additional factor in trying to repress the high blood lactate levels. This, is in contrast with previous findings where the MCT4 protein was $40 \%$ more abundant in a patient with mitochondrial myopathy [81], but the disease manifestation of this patient appears to be much milder and lactic acidosis may be less of a problem. Additionally, it has been reported that exercise training can increase the expression of

MCT4 [82]. Because of the strong muscle weakness in Leigh syndrome patients resulting in the inability of a normal active life and especially in the inability of physical exercise, this could also contribute to the down-regulation of the MCT4 gene.

\section{Alterations in protein turnover due to oxidative damage}

Protein turnover appears to be altered in muscle of SURF1 patients. The increase in protein synthesis is mainly based on the significant up-regulation of members of the $40 S$ (RPS11 and FAU) and $60 S$ (RPL3, RPL11, and RPL28) subunits of the cytoplasmic ribosomal proteins. In addition 14 transcripts coding for other subunits of the cytoplasmic ribosomal proteins tended to be more 
than 1.2 fold up-regulated and only one component of the 60S subunit was down-regulated, suggesting that the process as a whole appears to be up-regulated. The small sample size and the relatively small changes in gene expression could explain why the results for the majority of these genes were not significantly differentially expressed. On the other hand, there are also indications towards an increase in protein degradation by the proteasome, in particular the 205 proteasome. One transcript, encoding subunit PSMB9, was significantly up-regulated having a fold-change of 1.3, and six transcripts coding for other subunits of the 205 proteasome were up-regulated (> 1.2-fold), although not significantly. Moreover, one of the activators of the 20S complex (PSME1) was significantly up-regulated with a fold-change of 2.0. The $20 \mathrm{~S}$ complex of the proteasome is mainly associated with the breakdown of proteins with oxidative damage [83].

The increased production of reactive oxygen species (ROS) in the muscle of SURF1 patients is not uncommon for OXPHOS disease since impairment of the OXPHOS system can result in increased ROS production [32, 84]. Increased ROS production in the muscle of SURF1 patients is supported by a significant 1.8 fold up-regulation of glutathione peroxidase 3 (GPX3) and a significant 2.0 fold up-regulation of thioredoxin reductase 1 (TXNRD1), both having a protective effect as oxidoreductases. Although the apparent increase in oxidative protein damage observed in muscle of SURF1 patients was not statistically significant, SURF1 muscle cryosections contained significantly more enzymes able to produce superoxide radicals than the control samples, consistent with increased ROS production in muscle of SURF1 patients. We hypothesise that the increase in protein turnover due to the increased ROS production, results in efficient removal of the ROS damaged proteins.

\section{Increased expression of complement component genes}

A striking observation is the involvement of the immune system in SURF1 patients as illustrated by the up-regulation of a number of transcripts coding for complement components (table 4). Because of the physiological abundance of complement proteins, mainly produced by the liver and released in the serum, it was unexpected that the muscle itself showed an increased expression of complement components. The up-regulation of transcription of the $C_{1} Q B, C 1 R$ and C1S genes in SURF1 patients points towards the activation of complement via classical pathway $[85,86]$. The end-route of the complement cascade is usually the formation of the membrane attack complex (MAC) which leads to membrane leakage resulting in cell lyses. However, no difference in the amount of MAC for a SURF1 patient compared to an age-matched control could be identified by immunohistochemical staining against the complement $\mathrm{C} 5 \mathrm{~b}-9$ complex. Additionally, necrosis has not been described in muscle fibres of SURF1 patients [3]. This indicates that the activation of complement in muscle of SURF1 patients must have a different purpose than cell lyses. This was corroborated by the fact that no signs of inflammation were observed by haematoxylin and eosin staining (data not shown) and no gene expression changes were detected for macrophage or B-lymphocyte specific genes. Further support for an alternative 
explanation was based on comparing data from Duchene muscular dystrophy (DMD) models with our data, as up-regulation of complement components of the classical pathway has also been identified in mouse models for DMD [87, 88]. Like SURF1 patients, DMD patients and mouse models showed a reduction of the activities of the OXPHOS complexes, secondary to the primary cytoskeleton defect. These activities were decreased to 50\% of the normal activity in skeletal muscle (m.quadriceps) of dystrophin-deficient $\mathrm{mdx}$ mice [16). No signs of inflammation were observed in SURF1 patients, whereas infiltration of inflammatory cells has been identified in these mouse models [87]. Moreover, complement activation in DMD most likely results in necrosis of the muscle fibres [ 89,90$]$, which is not the case in muscle fibres of SURF1 patients. This is also illustrated by the difference in the immunohistochemical staining pattern for complement component C3C for a SURF1 patient, where C 3 C is mostly confined to muscle fibre membranes, and that of a muscle cryosection of a Duchene muscular dystrophy patient, which shows a more diffuse staining pattern (figure 1).

It has been reported that human skeletal myoblasts are able to express transcripts coding for complement components of the classical complement pathway themselves [91, 92]. The role of complement is broader than the immune system function alone and is also involved in bone and cartilage development, mammalian reproduction, and tissue and organ regeneration [93]. More specifically, complement component $C_{3}$ can have a specific tissue regenerative effect in the urodeles limb after amputation. Blastema cells (embryonic-like cells as precursors for regeneration) derived from muscle, which have the ability to differentiate into myotubes, express C3 in regenerating limbs of urodeles [94]. C3 has also been directly related to regeneration of the liver. After partial hepatectomy and after toxic injury $[95,96], \mathrm{C3}^{-1}$ mice showed a significant decrease in ability for liver regeneration, leading to an increased mortality. Rescuing of $\mathrm{C}_{3}^{-t}$ mice by using human or murine $\mathrm{C}_{3}$ or $\mathrm{C}_{3}$ a respectively, restored the regeneration process as illustrated by decreased parenchymal damage and increased hepatocyte proliferation. Similar observations were made in $\mathrm{C}^{-1-}$ mice treated with $\mathrm{CCl}_{4}$ resulting in toxic injury of the liver [95].

The possibility of a regeneration process in muscle of SURF1 patients was further supported by the microarray data. Two genes, interferon-related developmental regulator 1 (IFRD1) and insulinlike growth factor binding protein 3 (IGFBP3), which are involved in skeletal muscle development were both significantly up-regulated ( 1.7 and 4.5 fold respectively) in muscle of SURF1 patients. IFRD1 is classified in the gene-ontology biological process myoblast cell fate determination and it has been described that deprivation of the protein results in an inhibition of differentiation in myoblasts [24]. IGFBP3 is annotated as a positive regulator of myoblast differentiation and it has been reported that IGFBP3 has a role in the differentiation of primary human adult skeletal myoblasts [25]. Evidence for the occurrence of muscle development in our SURF1 patients is provided by the immunohistochemical data (figure 1), showing a positive staining of a number of muscle fibres for neonatal and foetal myosin. Finally, the PMP22 gene, which encodes an integral membrane protein being a major component of myelin in the peripheral nervous system, was 
significantly up-regulated. Various mutations in this gene are causative for Charcot-Marie-Tooth disease (MIM \#118220), which is a demyelinating neuropathy. The role of PMP22 in other tissues appears to be more complex and PMP22 has been shown to regulate cell growth [97]. Taking all data together, the complement activation in muscle of SURF1 patients most likely reflects a regeneration process rather than an immunological response, that might be triggered by the increased superoxide production of the impaired mitochondria and the resulting protein damage [98].

\section{Conclusion}

Mutations in the SURF1 gene lead to a defect in complex IV assembly and to a deficient OXPHOS system. Although this does not lead to major alterations in transcription of OXPHOS genes, it leads to significantly increased levels of ROS due to an increased expression of ROS producing enzymes, resulting in extensive protein damage. This may trigger the activation of on the one hand the $20 \mathrm{~S}$ complex of the proteasome to increase the capacity to breakdown damaged proteins and on the other hand of the complement system in turn inducing the process of muscle regeneration. A causative relation between these processes has to be established, but our data provide a new explanation on the pathological and rescue processes occurring in muscle of SURF1 patients, which can assist in finding therapeutic approaches. Whether the processes observed are common to other OXPHOS disorders or OXHPOS disorders in general remains to be determined. 
Supplementary table S1: QRT-PCR primers

\begin{tabular}{lll}
\hline Gene symbol & Forward primer (5'-3') & Reverse primer (5'-3') \\
\hline ARF1 & AATCAGCTCCGGACCAGAA & GCAGAGGGCAAGAGGAGGA \\
C1R & TCTGTGCTGGACACCCATCTC & GCAAAAACGCCCCCACTAT \\
C1S & TGGATAATGAAGACTATGCAGGAAA & GGAGAGGCTGGTGGGATGTA \\
C3 & TGGCCATTGAGCAGACCAT & CGTGCGCTGCTGTCCA \\
CLU & GCTGCAGGAATACCGCAAA & CCGTAGGTGCAAAAGCAACA \\
PKM2 & TCATTGCTGTGACCCGGA & ACAGGGAAGATGCCACGGTA \\
PMP22 & GGGACCGTGAGTTCCTAGAGC & CATTGCCAGACAGTCCTTGGA \\
RYR3 & AGGAGAGCACCGGGTATATGG & GACTAGAGAGATGATGGTATGGATGATG \\
SLC16A3 & GGGTGGGAACCGTGTCATT & GATGCCTTGTAACCTTGCGG \\
\hline
\end{tabular}

QRT-PCR primer sequences for ADP-ribosylation factor 1 (ARF1), complement component 1, $r$ subcomponent (C1R), complement component, s subcomponent (C1S), complement component $3\left(C_{3}\right)$, clusterin (CLU), pyruvate kinase, muscle (PKM2), peripheral myelin protein 22 (PMP22), ryanodine receptor 3 (RYR3), and solute carrier family 16, member 3 (SLC16A3). 
Supplementary Table S2: Differentially expressed genes

\begin{tabular}{|c|c|c|c|c|c|}
\hline Unigene ID & $\mathrm{FC}$ & Symbol & Name & GO Biological Process & GO Level \\
\hline Hs.436657 & 3.7 & CLU & Clusterin & complement activation; innate immune response & 5 \\
\hline Hs.155597 & 3.5 & CFD & Complement factor D (adipsin) & complement activation; innate immune response & 5 \\
\hline Hs.384598 & 2.7 & SERPING1 & Serpin peptidase inhibitor, clade $\mathrm{G}$ ( $\mathrm{C} 1$ inhibitor), member 1 , (angioedema, hereditary) & complement activation; innate immune response & 5 \\
\hline Hs.363396 & 2.6 & $\mathrm{CFH}$ & Complement factor $\mathrm{H}$ & complement activation; innate immune response & 5 \\
\hline Hs. 458355 & 2.3 & C1S & Complement component 1 , s subcomponent & complement activation; innate immune response & 5 \\
\hline Hs.8986 & 1.4 & $\mathrm{C} 1 \mathrm{QB}$ & Complement component 1, q subcomponent, B chain & complement activation; innate immune response & 5 \\
\hline Hs. 502705 & 1.4 & PRPF19 & PRP19/PSO4 pre-mRNA processing factor 19 homolog (S. cerevisiae) & DNA metabolism & 5 \\
\hline Hs.258429 & 1.4 & ERCC5 & $\begin{array}{l}\text { Excision repair cross-complementing rodent repair deficiency, complementation group } 5 \text { (xeroderma } \\
\text { pigmentosum, complementation group } \mathrm{G} \text { (Cockayne syndrome)) }\end{array}$ & DNA metabolism & 5 \\
\hline Hs.519474 & 1.4 & & $\begin{array}{l}\text { Transcribed locus, strongly similar to NP_001261.1 chromodomain helicase DNA binding protein } 1 \\
\text { [Homo sapiens] }\end{array}$ & DNA metabolism & 5 \\
\hline Hs.445078 & 1.3 & TLK2 & Tousled-like kinase 2 & DNA metabolism & 5 \\
\hline Hs.444118 & 1.3 & MCM6 & MCM6 minichromosome maintenance deficient 6 (MIS5 homolog, S. pombe) (S. cerevisiae) & DNA metabolism & 5 \\
\hline Hs. 475538 & 1.3 & XPC & Xeroderma pigmentosum, complementation group $\mathrm{C}$ & DNA metabolism & 5 \\
\hline Hs. 53454 & 1.2 & TOPBP1 & Topoisomerase (DNA) II binding protein 1 & DNA metabolism & 5 \\
\hline Hs. 88556 & 1.2 & HDAC1 & Histone deacetylase 1 & DNA metabolism & 5 \\
\hline Hs. 501522 & 1.2 & MGMT & 0-6-methylguanine-DNA methyltransferase & DNA metabolism & 5 \\
\hline Hs.269092 & 1.2 & CDYL & Chromodomain protein, Y-like & DNA metabolism & 5 \\
\hline Hs. 555936 & 1.2 & APEX2 & APEX nuclease (apurinic/apyrimidinic endonuclease) 2 & DNA metabolism & 5 \\
\hline Hs. 534331 & 1.2 & NUDT1 & Nudix (nucleoside diphosphate linked moiety X)-type motif 1 & DNA metabolism & 5 \\
\hline Hs.202672 & 1.1 & DNMT1 & DNA (cytosine-5-)-methyltransferase 1 & DNA metabolism & 5 \\
\hline Hs.1770 & 1.1 & LIG1 & Ligase I, DNA, ATP-dependent & DNA metabolism & 5 \\
\hline Hs.75307 & 1.3 & H1FX & H1 histone family, member $X$ & DNA metabolism; protein complex assembly & 5 \\
\hline Hs.76090 & 1.2 & TNFAIP1 & Tumor necrosis factor, alpha-induced protein 1 (endothelial) & DNA metabolism; protein complex assembly & 5 \\
\hline Hs. 406300 & 2.0 & RPL23 & Ribosomal protein $\mathrm{L} 23$ & nuclear transport & 5 \\
\hline Hs. 247077 & 1.7 & RHOA & Ras homolog gene family, member $A$ & nuclear transport & 5 \\
\hline Hs.12457 & 1.5 & NUP133 & Nucleoporin $133 \mathrm{kDa}$ & nuclear transport & 5 \\
\hline Hs. 523739 & 1.3 & STX5 & Syntaxin 5 & nuclear transport & 5 \\
\hline Hs. 501023 & 1.3 & MXI1 & MAX interactor 1 & nuclear transport & 5 \\
\hline Hs.430589 & 1.2 & CBLB & Cas-Br-M (murine) ecotropic retroviral transforming sequence b & nuclear transport & 5 \\
\hline Hs. 534373 & 1.4 & VAMP8 & Vesicle-associated membrane protein 8 (endobrevin) & protein complex assembly & 5 \\
\hline Hs.31334 & 1.4 & PRPF6 & PRP6 pre-mRNA processing factor 6 homolog (S. cerevisiae) & protein complex assembly & 5 \\
\hline Hs. 459759 & 1.3 & CREBBP & CREB binding protein (Rubinstein-Taybi syndrome) & protein complex assembly & 5 \\
\hline
\end{tabular}




\begin{tabular}{|c|c|c|c|}
\hline Hs.502872 & 1.2 & MAP3K11 & Mitogen-activated protein kinase kinase kinase 11 \\
\hline Hs. 87726 & 1.1 & GGA3 & Golgi associated, gamma adaptin ear containing, ARF binding protein 3 \\
\hline Hs.512464 & 0.4 & SURF1 & Surfeit 1 \\
\hline Hs. 450230 & 4.5 & IGFBP3 & Insulin-like growth factor binding protein 3 \\
\hline Hs. 4055 & 2.2 & KLF6 & Kruppel-like factor 6 \\
\hline Hs. 274313 & 1.6 & IGFBP6 & Insulin-like growth factor binding protein 6 \\
\hline Hs.67896 & 1.5 & OGFR & Opioid growth factor receptor \\
\hline Hs.235935 & 1.3 & NOV & Nephroblastoma overexpressed gene \\
\hline Hs. 435326 & 1.5 & ACTL6A & Actin-like $6 \mathrm{~A}$ \\
\hline Hs.528299 & 1.1 & HTATIP & HIV-1 Tat interacting protein, $60 \mathrm{kDa}$ \\
\hline Hs.23361 & 0.6 & ABTB2 & Ankyrin repeat and BTB (POZ) domain containing 2 \\
\hline Hs.65029 & 2.3 & GAS1 & Growth arrest-specific 1 \\
\hline Hs.282113 & 2.0 & SNF1LK & SNF1-like kinase \\
\hline Hs.153752 & 1.6 & CDC25B & Cell division cycle 25B \\
\hline Hs.371249 & 1.6 & PTN & Pleiotrophin (heparin binding growth factor 8 , neurite growth-promoting factor 1 ) \\
\hline Hs.504609 & 1.6 & ID1 & Inhibitor of DNA binding 1, dominant negative helix-loop-helix protein \\
\hline Hs.434286 & 1.5 & CHES1 & Checkpoint suppressor 1 \\
\hline Hs.106070 & 1.5 & CDKN1C & Cyclin-dependent kinase inhibitor 1C (p57, Kip2) \\
\hline Hs.82045 & 1.4 & MDK & Midkine (neurite growth-promoting factor 2) \\
\hline Hs.336994 & 1.4 & MTSS1 & Metastasis suppressor 1 \\
\hline Hs.483635 & 1.3 & FGF1 & Fibroblast growth factor 1 (acidic) \\
\hline Hs.152944 & 1.3 & LOH11CR2A & Loss of heterozygosity, 11 , chromosomal region 2 , gene A \\
\hline Hs.505033 & 1.2 & KRAS & V-Ki-ras2 Kirsten rat sarcoma viral oncogene homolog \\
\hline Hs.189772 & 1.2 & ССТ2 & Chaperonin containing TCP1, subunit 2 (beta) \\
\hline Hs.202453 & 1.2 & MYC & V-myc myelocytomatosis viral oncogene homolog (avian) \\
\hline Hs.239818 & 0.8 & PIK3CB & Phosphoinositide-3-kinase, catalytic, beta polypeptide \\
\hline Hs.438720 & 1.3 & MCM7 & MCM7 minichromosome maintenance deficient 7 (S. cerevisiae) \\
\hline Hs.156519 & 1.3 & MSH2 & MutS homolog 2, colon cancer, nonpolyposis type 1 (E. coli) \\
\hline Hs.240457 & 1.2 & RAD9A & RAD9 homolog A (S. pombe) \\
\hline Hs.446376 & 1.1 & APC2 & Adenomatosis polyposis coli 2 \\
\hline Hs.50130 & 2.5 & NDN & Necdin homolog (mouse) \\
\hline Hs.408312 & 1.1 & TP53 & Tumor protein p53 (Li-Fraumeni syndrome) \\
\hline
\end{tabular}

protein complex assembly

protein complex assembly

protein complex assembly

regulation of cell size; cell growth

regulation of cell size; cell growth

regulation of cell size; cell growth

regulation of cell size; cell growth

regulation of cell size; cell growth

regulation of cell size; cell growth; DNA metabolism

regulation of cell size; cell growth; DNA metabolism

regulation of cell size; cell growth; DNA metabolism; protein

complex assembly

regulation of progression through cell cycle

regulation of progression through cell cycle

regulation of progression through cell cycle

regulation of progression through cell cycle

regulation of progression through cell cycle

regulation of progression through cell cycle

regulation of progression through cell cycle

regulation of progression through cell cycle

regulation of progression through cell cycle

regulation of progression through cell cycle

regulation of progression through cell cycle

egulation of progression through cell cycle

regulation of progression through cell cycle

regulation of progression through cell cycle

regulation of progression through cell cycle

regulation of progression through cell cycle; DNA metabolism

egulation of progression through cell cycle; DNA metabolism

regulation of progression through cell cycle; DNA metabolism

regulation of progression through cell cycle; protein complex

assembly

regulation of progression through cell cycle; regulation of cell size;

cell growth

regulation of progression through cell cycle; regulation of cell size; $\quad 5$

cell growth; DNA metabolism; protein complex assembly 


\begin{tabular}{|c|c|c|c|}
\hline Hs. 512963 & 1.5 & ALG11 & Asparagine-linked glycosylation 11 homolog (S. cerevisiae, alpha-1,2-mannosyltransferase) \\
\hline Hs.389649 & 1.4 & DDX48 & DEAD (Asp-Glu-Ala-Asp) box polypeptide 48 \\
\hline Hs.75528 & 1.3 & GNL2 & Guanine nucleotide binding protein-like 2 (nucleolar) \\
\hline Hs. 10848 & 1.3 & BMS1L & BMS1-like, ribosome assembly protein (yeast) \\
\hline Hs.499620 & 1.2 & GEMIN4 & Gem (nuclear organelle) associated protein 4 \\
\hline Hs. 558447 & 1.2 & EMG1 & EMG1 nucleolar protein homolog (S. cerevisiae) \\
\hline Hs. 425777 & 1.8 & UBE2L6 & Ubiquitin-conjugating enzyme E2L 6 \\
\hline Hs. 121575 & 1.6 & CTSD & Cathepsin D (lysosomal aspartyl peptidase) \\
\hline Hs.398157 & 1.6 & PLK2 & Polo-like kinase 2 (Drosophila) \\
\hline Hs. 480415 & 1.6 & MANBA & Mannosidase, beta A, lysosomal \\
\hline Hs. 513617 & 1.5 & MMP2 & Matrix metallopeptidase 2 (gelatinase A, 72kDa gelatinase, $72 \mathrm{kDa}$ type IV collagenase) \\
\hline Hs.12813 & 1.5 & TIPARP & TCDD-inducible poly(ADP-ribose) polymerase \\
\hline Hs.65436 & 1.4 & LOXL1 & Lysyl oxidase-like 1 \\
\hline Hs. 184523 & 1.4 & STK38L & Serine/threonine kinase 38 like \\
\hline Hs. 532357 & 1.4 & TRIM21 & Tripartite motif-containing 21 \\
\hline Hs.387208 & 1.4 & FAU & $\begin{array}{l}\text { Finkel-Biskis-Reilly murine sarcoma virus (FBR-MuSV) ubiquitously expressed (fox derived); ribosomal } \\
\text { protein S30 }\end{array}$ \\
\hline Hs.154163 & 1.3 & PRMT2 & Protein arginine methyltransferase 2 \\
\hline Hs.524692 & 1.3 & NUAK1 & NUAK family, SNF1-like kinase, 1 \\
\hline Hs.529420 & 1.3 & UBE2G2 & Ubiquitin-conjugating enzyme E2G 2 (UBC7 homolog, yeast) \\
\hline Hs. 433728 & 1.2 & MAPK4 & Mitogen-activated protein kinase 4 \\
\hline Hs.234282 & 1.2 & VPS11 & Vacuolar protein sorting 11 (yeast) \\
\hline Hs.171596 & 1.2 & EPHA2 & EPH receptor A2 \\
\hline Hs.422662 & 1.1 & VRK1 & Vaccinia related kinase 1 \\
\hline Hs.59138 & 1.1 & GYPC & Glycophorin C (Gerbich blood group) \\
\hline Hs. 513926 & 1.1 & SENP3 & SUMO1/sentrin/SMT3 specific peptidase 3 \\
\hline Hs.95424 & 0.9 & MAP4K1 & Mitogen-activated protein kinase kinase kinase kinase 1 \\
\hline Hs.469809 & 0.8 & PTPN4 & Protein tyrosine phosphatase, non-receptor type 4 (megakaryocyte) \\
\hline Hs. 51133 & 0.8 & PTK6 & PTK6 protein tyrosine kinase 6 \\
\hline Hs.132760 & 0.7 & SLC37A4 & Solute carrier family 37 (glycerol-6-phosphate transporter), member 4 \\
\hline Hs.227777 & 0.4 & PTP4A1 & Protein tyrosine phosphatase type IVA, member 1 \\
\hline Hs.467097 & 2.1 & SNRP70 & Small nuclear ribonucleoprotein $70 \mathrm{kDa}$ polypeptide (RNP antigen) \\
\hline Hs.474018 & 1.7 & ADARB1 & Adenosine deaminase, RNA-specific, B1 (RED1 homolog rat) \\
\hline
\end{tabular}

ribosome biogenesis and assembly $\quad 5$

ribosome biogenesis and assembly

ribosome biogenesis and assembly

ribosome biogenesis and assembly

ribosome biogenesis and assembly $\quad 5$

ribosome biogenesis and assembly

biopolymer metabolism

biopolymer metabolism

biopolymer metabolism

biopolymer metabolism

biopolymer metabolism

biopolymer metabolism

biopolymer metabolism

biopolymer metabolism

biopolymer metabolism

biopolymer metabolism

biopolymer metabolism

biopolymer metabolism

biopolymer metabolism

biopolymer metabolism

biopolymer metabolism

biopolymer metabolism

biopolymer metabolism

biopolymer metabolism

biopolymer metabolism

biopolymer metabolism

biopolymer metabolism

biopolymer metabolism

biopolymer metabolism

biopolymer metabolism

bopolymer metabolism; nucleobase, nucleoside, nucleotide and ucleic acid metabolism

biopolymer metabolism; nucleobase, nucleoside, nucleotide and nucleic acid metabolism 


\begin{tabular}{|c|c|c|c|}
\hline Hs.493202 & 1.5 & CPSF1 & Cleavage and polyadenylation specific factor $1,160 \mathrm{kDa}$ \\
\hline Hs.83753 & 1.5 & SNRPB & Small nuclear ribonucleoprotein polypeptides B and B1 \\
\hline Hs.525006 & 1.4 & NUFIP1 & Nuclear fragile X mental retardation protein interacting protein 1 \\
\hline Hs.512610 & 1.3 & LSM7 & LSM7 homolog, U6 small nuclear RNA associated (S. cerevisiae) \\
\hline Hs.188879 & 1.2 & RBM6 & RNA binding motif protein 6 \\
\hline Hs.380277 & 1.3 & DAPK1 & Death-associated protein kinase 1 \\
\hline Hs.510528 & 1.1 & TRAF3 & TNF receptor-associated factor 3 \\
\hline Hs.183006 & 1.3 & GLCE & UDP-glucuronic acid epimerase \\
\hline Hs.522632 & 1.8 & TIMP1 & TIMP metallopeptidase inhibitor 1 \\
\hline Hs. 1735 & 3.1 & INHBB & Inhibin, beta B (activin AB beta polypeptide) \\
\hline Hs.370666 & 2.0 & FOX01A & Forkhead box $01 \mathrm{~A}$ (rhabdomyosarcoma) \\
\hline Hs.373550 & 1.4 & TGIF & TGFB-induced factor (TALE family homeobox) \\
\hline Hs.377090 & 1.3 & $\mathrm{ZHX} 2$ & Zinc fingers and homeoboxes 2 \\
\hline Hs.522373 & 3.0 & GSN & Gelsolin (amyloidosis, Finnish type) \\
\hline Hs.440829 & 4.6 & CEBPD & CCAAT/enhancer binding protein (C/EBP), delta \\
\hline Hs.513305 & 1.8 & TFAP4 & Transcription factor AP-4 (activating enhancer binding protein 4) \\
\hline Hs.462693 & 1.7 & ZNF22 & Zinc finger protein 22 (KOX 15) \\
\hline Hs.288856 & 1.6 & PFDN5 & Prefoldin subunit 5 \\
\hline Hs.14839 & 1.6 & POLR2G & Polymerase (RNA) II (DNA directed) polypeptide G \\
\hline Hs.76884 & 1.6 & ID3 & Inhibitor of DNA binding 3, dominant negative helix-loop-helix protein \\
\hline Hs.82071 & 1.6 & CITED2 & Cbp/p300-interacting transactivator, with Glu/Asp-rich carboxy-terminal domain, 2 \\
\hline Hs.522074 & 1.6 & TSC22D3 & TSC22 domain family, member 3 \\
\hline Hs.77890 & 1.5 & GUCY1B3 & Guanylate cyclase 1 , soluble, beta 3 \\
\hline Hs.194669 & 1.4 & EZH1 & Enhancer of zeste homolog 1 (Drosophila) \\
\hline
\end{tabular}

biopolymer metabolism; nucleobase, nucleoside, nucleotide and nucleic acid metabolism

biopolymer metabolism; nucleobase, nucleoside, nucleotide and nucleic acid metabolism

biopolymer metabolism; nucleobase, nucleoside, nucleotide and nucleic acid metabolism

biopolymer metabolism; nucleobase, nucleoside, nucleotide and 4 nucleic acid metabolism

biopolymer metabolism; nucleobase, nucleoside, nucleotide and 4 nucleic acid metabolism

biopolymer metabolism; positive regulation of cellular 4 physiological process

biopolymer metabolism; positive regulation of cellular 4 physiological process

cellular morphogenesis

cellular morphogenesis; regulation of cell proliferation; negative

regulation of cellular physiological process; positive regulation of 4 cellular physiological process

negative regulation of cellular physiological process

negative regulation of cellular physiological process; nucleobase,

nucleoside, nucleotide and nucleic acid metabolism

negative regulation of cellular physiological process; nucleobase, $\quad 4$

nucleoside, nucleotide and nucleic acid metabolism

negative regulation of cellular physiological process; nucleobase, $\quad 4$

nucleoside, nucleotide and nucleic acid metabolism

negative regulation of cellular physiological process; organelle 4 organization and biogenesis

nucleobase, nucleoside, nucleotide and nucleic acid metabolism 4

nucleobase, nucleoside, nucleotide and nucleic acid metabolism 4

nucleobase, nucleoside, nucleotide and nucleic acid metabolism 4

nucleobase, nucleoside, nucleotide and nucleic acid metabolism $\quad 4$

nucleobase, nucleoside, nucleotide and nucleic acid metabolism $\quad 4$

nucleobase, nucleoside, nucleotide and nucleic acid metabolism $\quad 4$

nucleobase, nucleoside, nucleotide and nucleic acid metabolism $\quad 4$

nucleobase, nucleoside, nucleotide and nucleic acid metabolism $\quad 4$

nucleobase, nucleoside, nucleotide and nucleic acid metabolism 4

nucleobase, nucleoside, nucleotide and nucleic acid metabolism 


\begin{tabular}{|c|c|c|c|c|}
\hline Hs.43697 & 1.3 & ETV5 & Ets variant gene 5 (ets-related molecule) & nucleobase, nucleoside, nucleotide and nucleic acid metabolism \\
\hline Hs.327527 & 1.3 & SMARCA4 & SWI/SNF related, matrix associated, actin dependent regulator of chromatin, subfamily a, member 4 & nucleobase, nucleoside, nucleotide and nucleic acid metabolism \\
\hline Hs.532277 & 1.3 & ZNF250 & Zinc finger protein 250 & nucleobase, nucleoside, nucleotide and nucleic acid metabolism \\
\hline Hs.200250 & 1.3 & CREM & CAMP responsive element modulator & nucleobase, nucleoside, nucleotide and nucleic acid metabolism \\
\hline Hs.388236 & 1.2 & $\mathrm{ClC}$ & Capicua homolog (Drosophila) & nucleobase, nucleoside, nucleotide and nucleic acid metabolism \\
\hline Hs.273621 & 1.2 & CNP & 2',3'-cyclic nucleotide 3' phosphodiesterase & nucleobase, nucleoside, nucleotide and nucleic acid metabolism \\
\hline Hs.505004 & 1.2 & TCEA2 & Transcription elongation factor A (SII), 2 & nucleobase, nucleoside, nucleotide and nucleic acid metabolism \\
\hline Hs.584839 & 1.2 & POLR1C & Polymerase (RNA) I polypeptide C, 30kDa & nucleobase, nucleoside, nucleotide and nucleic acid metabolism \\
\hline Hs.371987 & 1.2 & NFAT5 & Nuclear factor of activated T-cells 5 , tonicity-responsive & nucleobase, nucleoside, nucleotide and nucleic acid metabolism \\
\hline Hs.89781 & 1.2 & UBTF & Upstream binding transcription factor, RNA polymerase I & nucleobase, nucleoside, nucleotide and nucleic acid metabolism \\
\hline Hs.440219 & 1.1 & UBN1 & Ubinuclein 1 & nucleobase, nucleoside, nucleotide and nucleic acid metabolism \\
\hline Hs.158174 & 1.1 & ZNF184 & Zinc finger protein 184 (Kruppel-like) & nucleobase, nucleoside, nucleotide and nucleic acid metabolism \\
\hline Hs.432574 & 1.1 & POLR2H & Polymerase (RNA) II (DNA directed) polypeptide H & nucleobase, nucleoside, nucleotide and nucleic acid metabolism \\
\hline Hs.124553 & 1.1 & ZNF263 & Zinc finger protein 263 & nucleobase, nucleoside, nucleotide and nucleic acid metabolism \\
\hline Hs.409876 & 1.1 & ZBTB24 & Zinc finger and BTB domain containing 24 & nucleobase, nucleoside, nucleotide and nucleic acid metabolism \\
\hline Hs.21771 & 1.1 & WHSC2 & Wolf-Hirschhorn syndrome candidate 2 & nucleobase, nucleoside, nucleotide and nucleic acid metabolism \\
\hline Hs.178728 & 0.8 & MBD3 & Methyl-CpG binding domain protein 3 & nucleobase, nucleoside, nucleotide and nucleic acid metabolism \\
\hline Hs.515053 & 0.8 & AES & Amino-terminal enhancer of split & nucleobase, nucleoside, nucleotide and nucleic acid metabolism \\
\hline Hs.332173 & 0.7 & TLE2 & Transducin-like enhancer of split 2 (E(sp1) homolog, Drosophila) & nucleobase, nucleoside, nucleotide and nucleic acid metabolism \\
\hline Hs.518249 & 0.6 & CNBP & Zinc finger protein 9 & nucleobase, nucleoside, nucleotide and nucleic acid metabolism \\
\hline Hs.300701 & 1.9 & TUBB2B & Tubulin, beta 2B & organelle organization and biogenesis \\
\hline Hs.444767 & 1.5 & KIF13B & Kinesin family member 13B & organelle organization and biogenesis \\
\hline Hs.325528 & 1.2 & & $\begin{array}{l}\text { Transcribed locus, strongly similar to NP_004937.1 dedicator of cytokinesis 2; dedicator of cyto-kinesis } 2 \\
\text { [Homo sapiens] }\end{array}$ & organelle organization and biogenesis \\
\hline Hs.138378 & 1.8 & CASP4 & Caspase 4 , apoptosis-related cysteine peptidase & positive regulation of cellular physiological process \\
\hline Hs. 432330 & 0.8 & RRAGA & Ras-related GTP binding A & positive regulation of cellular physiological process \\
\hline Hs.367725 & 1.4 & GATA2 & GATA binding protein 2 & $\begin{array}{l}\text { positive regulation of cellular physiological process; nucleobase, } \\
\text { nucleoside, nucleotide and nucleic acid metabolism }\end{array}$ \\
\hline Hs.459070 & 0.5 & ARNT2 & Aryl-hydrocarbon receptor nuclear translocator 2 & $\begin{array}{l}\text { positive regulation of cellular physiological process; nucleobase, } \\
\text { nucleoside, nucleotide and nucleic acid metabolism } \\
\text { regulation of cell cycle; DNA repair; negative regulation of cellular }\end{array}$ \\
\hline Hs.1565 & 0.6 & NEDD4 & Neural precursor cell expressed, developmentally down-regulated 4 & $\begin{array}{l}\text { physiological process; biopolymer metabolism; biopolymer } \\
\text { metabolism; nucleobase, nucleoside, nucleotide and nucleic acid } \\
\text { metabolism }\end{array}$ \\
\hline Hs.372031 & 4.9 & PMP22 & Peripheral myelin protein 22 & $\begin{array}{l}\text { regulation of cell proliferation; negative regulation of cellular } \\
\text { physiological process }\end{array}$ \\
\hline
\end{tabular}

4




\begin{tabular}{|c|c|c|c|}
\hline Hs.17466 & 1.9 & RARRES3 & Retinoic acid receptor responder (tazarotene induced) 3 \\
\hline Hs. 512680 & 1.4 & CLEC11A & C-type lectin domain family 11 , member A \\
\hline Hs.514581 & 2.2 & ACTG1 & Actin, gamma 1 \\
\hline Hs.469615 & 1.7 & sep-10 & Septin 10 \\
\hline Hs. 244580 & 1.2 & TPX2 & TPX2, microtubule-associated, homolog (Xenopus laevis) \\
\hline Hs.522114 & 1.6 & CLTA & Clathrin, light polypeptide (Lca) \\
\hline Hs.441498 & 1.3 & STAM & Signal transducing adaptor molecule (SH3 domain and ITAM motif) 1 \\
\hline Hs.464779 & 1.3 & NPC1 & Niemann-Pick disease, type C1 \\
\hline Hs.531752 & 1.1 & RANBP3 & RAN binding protein 3 \\
\hline Hs.531561 & 1.8 & EMP2 & Epithelial membrane protein 2 \\
\hline Hs.315177 & 0.8 & IFRD2 & Interferon-related developmental regulator 2 \\
\hline Hs.567497 & 3.1 & C1R & Complement component 1 , r subcomponent \\
\hline Hs.356794 & 1.8 & RPS24 & Ribosomal protein S24 \\
\hline Hs.496593 & 1.7 & CAPN6 & Calpain 6 \\
\hline Hs.433529 & 1.6 & RPS11 & Ribosomal protein S11 \\
\hline Hs.516493 & 1.6 & FAP & Fibroblast activation protein, alpha \\
\hline Hs.408073 & 1.6 & RPS6 & Ribosomal protein S6 \\
\hline Hs.429180 & 1.5 & EIF2S2 & Eukaryotic translation initiation factor 2 , subunit 2 beta, $38 \mathrm{kDa}$ \\
\hline Hs.477891 & 1.4 & CPB1 & Carboxypeptidase B1 (tissue) \\
\hline Hs.438429 & 1.4 & RPS19 & Ribosomal protein S19 \\
\hline Hs.419240 & 1.3 & SLC2A14 & Solute carrier family 2 (facilitated glucose transporter), member 14 \\
\hline Hs.436405 & 1.2 & IDH3B & Isocitrate dehydrogenase 3 (NAD+) beta \\
\hline Hs.75859 & 1.2 & MRPL49 & Mitochondrial ribosomal protein L49 \\
\hline Hs.412433 & 1.2 & AIP & Aryl hydrocarbon receptor interacting protein \\
\hline Hs.91161 & 1.2 & PFDN4 & Prefoldin subunit 4 \\
\hline Hs.500156 & 1.1 & DNAJC7 & DnaJ (Hsp40) homolog, subfamily C, member 7 \\
\hline Hs.54470 & 0.9 & ABCC8 & ATP-binding cassette, sub-family C (CFTR/MRP), member 8 \\
\hline Hs.298469 & 0.9 & ACE & Angiotensin I converting enzyme (peptidyl-dipeptidase A) 1 \\
\hline Hs.179704 & 0.7 & MEP1A & Meprin A, alpha (PABA peptide hydrolase) \\
\hline Hs.466471 & 0.7 & GPI & Glucose phosphate isomerase \\
\hline Hs.501200 & 1.6 & RGS10 & Regulator of G-protein signalling 10 \\
\hline Hs.9914 & 1.8 & FST & Follistatin \\
\hline
\end{tabular}

regulation of cell proliferation; negative regulation of cellular physiological process

regulation of cell proliferation; positive regulation of cellular 4 physiological process

cell cycle

cell cycle

cell cycle; cell proliferation

cell organization and biogenesis

cell organization and biogenesis

cell organization and biogenesis

cell organization and biogenesis

cell proliferation

cell proliferation

macromolecule metabolism; primary metabolism

macromolecule metabolism; primary metabolism

macromolecule metabolism; primary metabolism

macromolecule metabolism; primary metabolism

macromolecule metabolism; primary metabolism

macromolecule metabolism; primary metabolism

macromolecule metabolism; primary metabolism

macromolecule metabolism; primary metabolism

macromolecule metabolism; primary metabolism

macromolecule metabolism; primary metabolism

macromolecule metabolism; primary metabolism

macromolecule metabolism; primary metabolism

macromolecule metabolism; primary metabolism

macromolecule metabolism; primary metabolism

macromolecule metabolism; primary metabolism

macromolecule metabolism; primary metabolism

macromolecule metabolism; primary metabolism

macromolecule metabolism; primary metabolism

macromolecule metabolism; primary metabolism

negative regulation of cellular process

negative regulation of cellular process; negative regulation of

physiological process 


\begin{tabular}{|c|c|c|c|}
\hline Hs. 443625 & 3.7 & COL3A1 & Collagen, type III, alpha 1 (Ehlers-Danlos syndrome type IV, autosomal dominant) \\
\hline Hs.409034 & 2.2 & COL15A1 & Collagen, type XV, alpha 1 \\
\hline Hs.82116 & 1.8 & MYD88 & Myeloid differentiation primary response gene (88) \\
\hline Hs.1116 & 1.2 & LTBR & Lymphotoxin beta receptor (TNFR superfamily, member 3) \\
\hline Hs.405156 & 2.0 & PPAP2B & Phosphatidic acid phosphatase type 2B \\
\hline Hs.200136 & 1.8 & ACAA2 & Acetyl-Coenzyme A acyltransferase 2 (mitochondrial 3-oxoacyl-Coenzyme A thiolase) \\
\hline Hs.533514 & 1.7 & ASMTL & Acetylserotonin O-methyltransferase-like \\
\hline Hs. 195040 & 1.5 & HSD11B1 & Hydroxysteroid (11-beta) dehydrogenase 1 \\
\hline Hs.512670 & 1.3 & BCAT2 & Branched chain aminotransferase 2 , mitochondrial \\
\hline Hs.467554 & 1.1 & TPO & Thyroid peroxidase \\
\hline Hs.319438 & 1.1 & PLA2G5 & Phospholipase A2, group V \\
\hline Hs.75069 & 1.1 & SHMT2 & Serine hydroxymethyltransferase 2 (mitochondrial) \\
\hline Hs.481551 & 0.8 & MTRR & 5-methyltetrahydrofolate-homocysteine methyltransferase reductase \\
\hline Hs.76244 & 0.7 & SRM & Spermidine synthase \\
\hline Hs.200841 & 1.6 & LAMA2 & Laminin, alpha 2 (merosin, congenital muscular dystrophy) \\
\hline Hs.192233 & 2.7 & PPL & Periplakin \\
\hline Hs.516505 & 1.6 & S100A13 & S100 calcium binding protein A13 \\
\hline Hs.495710 & 1.5 & GPM6B & Glycoprotein M6B \\
\hline Hs.7879 & 1.7 & IFRD1 & Interferon-related developmental regulator 1 \\
\hline Hs.533317 & 3.7 & VIM & Vimentin \\
\hline Hs.508716 & 3.1 & COL4A2 & Collagen, type IV, alpha 2 \\
\hline Hs.514412 & 2.2 & PECAM1 & Platelet/endothelial cell adhesion molecule (CD31 antigen) \\
\hline Hs.519909 & 2.0 & MARCKS & Myristoylated alanine-rich protein kinase $\mathrm{C}$ substrate \\
\hline Hs.529571 & 2.0 & RBP1 & Retinol binding protein 1 , cellular \\
\hline Hs.58351 & 2.0 & $\mathrm{ABCA8}$ & ATP-binding cassette, sub-family A ( $A B C 1$ ), member 8 \\
\hline Hs. 567352 & 1.9 & TXNRD1 & Thioredoxin reductase 1 \\
\hline Hs.513058 & 1.6 & TMED3 & Transmembrane emp24 protein transport domain containing 3 \\
\hline Hs.77422 & 1.5 & PLP2 & Proteolipid protein 2 (colonic epithelium-enriched) \\
\hline Hs.47629 & 1.5 & COL21A1 & Collagen, type XXI, alpha 1 \\
\hline Hs.474783 & 1.4 & TST & Thiosulfate sulfurtransferase (rhodanese) \\
\hline Hs.501868 & 1.4 & TMEM41B & Transmembrane protein 41B \\
\hline Hs.32309 & 1.3 & INPP1 & Inositol polyphosphate-1-phosphatase \\
\hline Hs.472270 & 1.3 & RIN2 & Ras and Rab interactor 2 \\
\hline Hs. 253420 & 0.8 & NOS1 & Nitric oxide synthase 1 (neuronal) \\
\hline Hs.323878 & 0.7 & SLC1A4 & Solute carrier family 1 (glutamate/neutral amino acid transporter), member 4 \\
\hline
\end{tabular}

\begin{tabular}{ll} 
organ morphogenesis & 3 \\
organ morphogenesis & 3 \\
positive regulation of cellular process & 3 \\
positive regulation of cellular process & 3 \\
primary metabolism & 3 \\
primary metabolism & 3 \\
primary metabolism & 3 \\
primary metabolism & 3 \\
primary metabolism & 3 \\
primary metabolism & 3 \\
primary metabolism & 3 \\
primary metabolism & 3 \\
primary metabolism & 3 \\
primary metabolism & 3 \\
regulation of cellular physiological process & 3 \\
cell differentiation & 3 \\
cell differentiation & 2 \\
cell differentiation & 2 \\
cell differentiation; organ development & 2 \\
cellular physiological process & 2 \\
cellular physiological process & 2 \\
cellular physiological process & 2 \\
cellular physiological process & 2 \\
cellular physiological process & 2 \\
cellular physiological process & 2 \\
cellular physiological process & 2 \\
cellular physiological process & 2 \\
cellular physiological process & 2 \\
cellular physiological process & 2 \\
cellular physiological process & 2 \\
cellular physiological process & 2 \\
cellular physiological process & 2 \\
cellular physiological process & 2 \\
cellular physiological process & 2 \\
cellular physiological process & 2 \\
\hline
\end{tabular}




\begin{tabular}{|c|c|c|c|}
\hline Hs.587054 & 0.7 & G3BP & Ras-GTPase-activating protein SH3-domain-binding protein \\
\hline Hs.369250 & 0.4 & RYR3 & Ryanodine receptor 3 \\
\hline Hs.500761 & 0.4 & SLC16A3 & Solute carrier family 16 (monocarboxylic acid transporters), member 3 \\
\hline Hs.200629 & 0.7 & KCNJ12 & Potassium inwardly-rectifying channel, subfamily J, member 12 \\
\hline Hs. 476092 & 3.3 & CLEC3B & C-type lectin domain family 3 , member B \\
\hline Hs. 515369 & 1.9 & TYROBP & TYRO protein tyrosine kinase binding protein \\
\hline Hs. 325978 & 1.3 & NUMA1 & Nuclear mitotic apparatus protein 1 \\
\hline Hs.1570 & 1.3 & HRH1 & Histamine receptor $\mathrm{H} 1$ \\
\hline Hs. 275775 & 2.5 & SEPP1 & Selenoprotein P, plasma, 1 \\
\hline Hs.386793 & 1.8 & GPX3 & Glutathione peroxidase 3 (plasma) \\
\hline Hs.2030 & 1.5 & THBD & Thrombomodulin \\
\hline Hs. 153381 & 2.5 & DARC & Duffy blood group, chemokine receptor \\
\hline Hs. 505337 & 2.3 & CLDN5 & Claudin 5 (transmembrane protein deleted in velocardiofacial syndrome) \\
\hline Hs.356624 & 2.0 & NID1 & Nidogen 1 \\
\hline Hs. 471751 & 1.8 & CMKOR1 & Chemokine orphan receptor 1 \\
\hline Hs.2465 & 1.6 & P2RY14 & Purinergic receptor P2Y, G-protein coupled, 14 \\
\hline Hs.421986 & 1.5 & CXCR4 & Chemokine (C-X-C motif) receptor 4 \\
\hline Hs. 514242 & 1.4 & PLEKHM1 & Pleckstrin homology domain containing, family M (with RUN domain) member 1 \\
\hline Hs. 109225 & 1.4 & VCAM1 & Vascular cell adhesion molecule 1 \\
\hline Hs. 444362 & 1.3 & LPP & LIM domain containing preferred translocation partner in lipoma \\
\hline Hs.56045 & 1.2 & STAC & $\mathrm{SH} 3$ and cysteine rich domain \\
\hline Hs. 433300 & 1.2 & FCER1G & Fc fragment of IgE, high affinity I, receptor for; gamma polypeptide \\
\hline Hs.500771 & 1.2 & DNMBP & Dynamin binding protein \\
\hline Hs. 529846 & 1.2 & CAMLG & Calcium modulating ligand \\
\hline Hs. 449076 & 1.2 & PWP2H & PWP2 periodic tryptophan protein homolog (yeast) \\
\hline Hs.67846 & 1.1 & LILRB1 & Leukocyte immunoglobulin-like receptor, subfamily B (with TM and ITIM domains), member 1 \\
\hline Hs. 529449 & 0.9 & APBB3 & Amyloid beta (A4) precursor protein-binding, family B, member 3 \\
\hline Hs. 523360 & 0.9 & INPP5A & Inositol polyphosphate-5-phosphatase, 40kDa \\
\hline Hs. 519873 & 1.5 & DSP & Desmoplakin \\
\hline Hs.97616 & 1.3 & SH3GL1 & SH3-domain GRB2-like 1 \\
\hline Hs.434971 & 1.2 & TRO & Trophinin \\
\hline Hs.524116 & 1.2 & NRGN & Neurogranin (protein kinase C substrate, RC3) \\
\hline Hs. 440168 & 0.6 & DSCR1L1 & Down syndrome critical region gene 1-like 1 \\
\hline Hs. 436204 & 1.4 & ZNF289 & Zinc finger protein 289, ID1 regulated \\
\hline Hs.485717 & 0.9 & SMAP1 & Stromal membrane-associated protein 1 \\
\hline
\end{tabular}

\begin{tabular}{ll} 
cellular physiological process & 2 \\
cellular physiological process & 2 \\
cellular physiological process & 2 \\
cellular physiological process; regulation of physiological process & 2 \\
organ development & 2 \\
response to stress & 2 \\
response to stress & 2 \\
response to stress & 2 \\
response to stress; cellular physiological process & 2 \\
response to stress; cellular physiological process & 2 \\
response to stress; cellular physiological process & 2 \\
cellular process & 1 \\
cellular process & 1 \\
cellular process & 1 \\
cellular process & 1 \\
cellular process & 1 \\
cellular process & 1 \\
cellular process & 1 \\
cellular process & 1 \\
cellular process & 1 \\
cellular process & 1 \\
cellular process & 1 \\
cellular process & 1 \\
cellular process & 1 \\
cellular process & 1 \\
cellular process & 1 \\
cellular process & 1 \\
cellular process & 1 \\
development & 1 \\
development; cellular process & 1 \\
development; cellular process & 1 \\
development; cellular process & 1 \\
development; cellular process & 1 \\
regulation of biological process & 1 \\
regulation of biological process & 1 \\
\hline
\end{tabular}




\begin{tabular}{llll} 
Hs.503165 & 1.3 & CENTD2 & Centaurin, delta 2 \\
Hs.503911 & 7.1 & NNMT & Nicotinamide N-methyltransferase \\
Hs.593775 & 4.7 & & Data not found \\
Hs.604114 & 3.6 & & Transcribed locus \\
Hs.597039 & 2.8 & & Transcribed locus \\
Hs.593731 & 2.6 & & Transcribed locus \\
Hs.523012 & 2.6 & DDIT4 & DNA-damage-inducible transcript 4 \\
Hs.503709 & 2.6 & TMEM123 & Transmembrane protein 123 \\
Hs.630569 & 2.5 & & Transcribed locus \\
Hs.85155 & 2.4 & ZFP36L1 & Zinc finger protein 36, C3H type-like 1 \\
Hs.458573 & 2.4 & PDGFRL & Platelet-derived growth factor receptor-like \\
Hs.6434 & 2.4 & C14orf132 & Chromosome 14 open reading frame 132 \\
Hs.533977 & 2.1 & TXNIP & Thioredoxin interacting protein \\
Hs.633687 & 2.1 & & Transcribed locus \\
Hs.630420 & 2.0 & & Transcribed locus \\
Hs.75348 & 2.0 & PSME1 & Proteasome (prosome, macropain) activator subunit 1 (PA28 alpha) \\
Hs.465761 & 1.9 & ARHGEF18 & Rho/rac guanine nucleotide exchange factor (GEF) 18 \\
Hs.523414 & 1.9 & IGF2 & Insulin-like growth factor 2 (somatomedin A) \\
Hs.522665 & 1.8 & MAGED2 & Melanoma antigen family D, 2 \\
Hs.376046 & 1.7 & BTN3A2 & Butyrophilin, subfamily 3, member A2 \\
Hs.594675 & 1.7 & & Transcribed locus \\
Hs.150718 & 1.7 & JAM3 & Junctional adhesion molecule 3 \\
Hs.642681 & 1.7 & & Data not found \\
Hs.633245 & 1.6 & & Data not found \\
Hs.631504 & 1.5 & RASSF2 & Ras association (RalGDS/AF-6) domain family 2 \\
Hs.525462 & 1.5 & C1orf41 & Chromosome 1 open reading frame 41 \\
Hs.591258 & 1.5 & CD74 & CD74 molecule, major histocompatibility complex, class II invariant chain \\
Hs.632486 & 1.5 & MCL1 & Myeloid cell leukemia sequence 1 (BCL2-related) \\
Hs.412117 & 1.5 & ANXA6 & Annexin A6 \\
Hs.602328 & 1.5 & & Transcribed locus \\
Hs.144492 & 1.5 & PLCE1 & Phospholipase C, epsilon 1 \\
Hs.434219 & 1.5 & ANKHD1 & Ankyrin repeat and KH domain containing 1 \\
Hs.592158 & 1.5 & & Data not found \\
Hs.603387 & 1.5 & & Transcribed locus \\
Hs.562227 & 1.5 & HSPG2 & Heparan sulfate proteoglycan 2 (perlecan) \\
& & & \\
\hline
\end{tabular}

regulation of biological process; cellular process

NA

NA

NA

NA

NA

NA

NA

NA

NA

NA

NA

NA

NA

NA

NA

NA

NA

NA

NA

NA

NA

NA

NA

NA

NA

NA

NA

NA

NA

NA

NA

NA

NA

NA 


\begin{tabular}{|c|c|c|c|}
\hline Hs.592182 & 1.5 & AMPH & Amphiphysin (Stiff-Man syndrome with breast cancer 128kDa autoantigen) \\
\hline Hs.520102 & 1.4 & KIAA0082 & KIAA0082 \\
\hline Hs. 632776 & 1.4 & C22orf28 & Hypothetical protein HSPC117 \\
\hline Hs. 429434 & 1.4 & GAB2 & GRB2-associated binding protein 2 \\
\hline Hs.194816 & 1.4 & STOML1 & Stomatin (EPB72)-like 1 \\
\hline Hs.304682 & 1.4 & CST3 & Cystatin C (amyloid angiopathy and cerebral hemorrhage) \\
\hline Hs. 195740 & 1.4 & AATF & Apoptosis antagonizing transcription factor \\
\hline Hs. 508010 & 1.4 & FNDC3A & Fibronectin type III domain containing $3 \mathrm{~A}$ \\
\hline Hs.279902 & 1.4 & CRSP9 & Cofactor required for Sp1 transcriptional activation, subunit 9, 33kDa \\
\hline Hs. 617685 & 1.4 & & Transcribed locus \\
\hline Hs.592088 & 1.4 & ZNF205 & Zinc finger protein 205 \\
\hline Hs.593551 & 1.4 & & Transcribed locus \\
\hline Hs. 447458 & 1.4 & C10orf6 & Chromosome 10 open reading frame 6 \\
\hline Hs.20013 & 1.4 & SYF2 & SYF2 homolog, RNA splicing factor (S. cerevisiae) \\
\hline Hs.637254 & 1.4 & & Transcribed locus \\
\hline Hs. 556496 & 1.4 & TANK & TRAF family member-associated NFKB activator \\
\hline Hs.592324 & 1.4 & PSEN1 & Presenilin 1 (Alzheimer disease 3) \\
\hline Hs. 592044 & 1.4 & RSL1D1 & Ribosomal L1 domain containing 1 \\
\hline Hs.198853 & 1.3 & C12orf32 & Chromosome 12 open reading frame 32 \\
\hline Hs. 47338 & 1.3 & IFIT3 & Interferon-induced protein with tetratricopeptide repeats 3 \\
\hline Hs.103834 & 1.3 & TMEM106C & Transmembrane protein $106 \mathrm{C}$ \\
\hline Hs. 643014 & 1.3 & & Transcribed locus \\
\hline Hs.98041 & 1.3 & ZFYVE26 & Zinc finger, FYVE domain containing 26 \\
\hline Hs.632264 & 1.3 & TGIF2 & TGFB-induced factor 2 (TALE family homeobox) \\
\hline Hs.631844 & 1.3 & DAPK3 & Death-associated protein kinase 3 \\
\hline Hs.484242 & 1.3 & UBXD8 & UBX domain containing 8 \\
\hline Hs.597347 & 1.3 & & Transcribed locus \\
\hline Hs.595312 & 1.3 & & Transcribed locus \\
\hline Hs. 99093 & 1.3 & ZNF428 & Chromosome 19 open reading frame 37 \\
\hline Hs. 458644 & 1.3 & R3HCC1 & R3H domain and coiled-coil containing 1 \\
\hline Hs.591457 & 1.3 & POLR3C & Polymerase (RNA) III (DNA directed) polypeptide C (62kD) \\
\hline Hs.631919 & 1.3 & USP4 & Ubiquitin specific peptidase 4 (proto-oncogene) \\
\hline Hs.622982 & 1.3 & & Transcribed locus \\
\hline Hs. 37616 & 1.3 & STRA13 & Stimulated by retinoic acid 13 homolog (mouse) \\
\hline Hs.594086 & 1.2 & & Transcribed locus \\
\hline
\end{tabular}

$\begin{array}{lll}\text { NA } & 0 & ? \\ \text { NA } & 0 & \text { 章 } \\ \text { NA } & 0 & \text { 罗 } \\ \text { NA } & 0 & \text { w }\end{array}$

Hs.594086 $\quad 1.2$

Transcribed locus 


\begin{tabular}{|c|c|c|c|}
\hline Hs.104661 & 1.2 & C19orf7 & Chromosome 19 open reading frame 7 \\
\hline Hs.99821 & 1.2 & C10orf22 & Chromosome 10 open reading frame 22 \\
\hline Hs.461860 & 1.2 & NUP214 & Nucleoporin 214kDa \\
\hline Hs.466714 & 1.2 & PAF1 & Paf1, RNA polymerase II associated factor, homolog (S. cerevisiae) \\
\hline Hs.65234 & 1.2 & DDX27 & DEAD (Asp-Glu-Ala-Asp) box polypeptide 27 \\
\hline Hs.591449 & 1.2 & MTF2 & Metal response element binding transcription factor 2 \\
\hline Hs.493309 & 1.2 & KIAA0020 & KIAA0020 \\
\hline Hs.89497 & 1.2 & LMNB1 & Lamin B1 \\
\hline Hs.522662 & 1.2 & TSR2 & Hypothetical protein DT1P1A10 \\
\hline Hs.613614 & 1.2 & & Transcribed locus \\
\hline Hs.634602 & 1.2 & & Transcribed locus \\
\hline Hs.433269 & 1.2 & C14orf11 & Chromosome 14 open reading frame 11 \\
\hline Hs.191046 & 1.2 & PDE1A & Phosphodiesterase $1 \mathrm{~A}$, calmodulin-dependent \\
\hline Hs.606668 & 1.2 & & Transcribed locus \\
\hline Hs.591036 & 1.2 & MDM1 & Mdm4, transformed $3 T 3$ cell double minute 1, p53 binding protein (mouse) \\
\hline Hs.592298 & 1.2 & PPM1A & Protein phosphatase $1 \mathrm{~A}$ (formerly $2 \mathrm{C}$ ), magnesium-dependent, alpha isoform \\
\hline Hs.352614 & 1.2 & AGPAT7 & 1-acylglycerol-3-phosphate 0 -acyltransferase 7 (lysophosphatidic acid acyltransferase, eta) \\
\hline Hs. 557646 & 1.2 & CDK9 & Cyclin-dependent kinase 9 (CDC2-related kinase) \\
\hline Hs.567236 & 1.2 & SHROOM2 & Apical protein-like (Xenopus laevis) \\
\hline Hs. 458360 & 1.2 & UCK2 & Uridine-cytidine kinase 2 \\
\hline Hs. 642711 & 1.2 & & Data not found \\
\hline Hs. 512627 & 1.2 & INTS10 & Integrator complex subunit 10 \\
\hline Hs.21811 & 1.1 & $\operatorname{CCDC94}$ & Coiled-coil domain containing 94 \\
\hline Hs.9043 & 1.1 & C14orf120 & Chromosome 14 open reading frame 120 \\
\hline Hs.618686 & 1.1 & & Transcribed locus \\
\hline Hs.596659 & 0.9 & & Data not found \\
\hline Hs. 517331 & 0.9 & C21orf2 & Chromosome 21 open reading frame 2 \\
\hline Hs.519702 & 0.9 & CYFIP2 & Cytoplasmic FMR1 interacting protein 2 \\
\hline Hs.213666 & 0.9 & KIAA0460 & KIAA0460 \\
\hline Hs.603821 & 0.9 & & Transcribed locus \\
\hline Hs.460499 & 0.9 & ATXN2L & Ataxin 2-like \\
\hline Hs. 440364 & 0.8 & DYNC111 & Dynein, cytoplasmic 1, intermediate chain 1 \\
\hline Hs.491354 & 0.8 & EXTL3 & Exostoses (multiple)-like 3 \\
\hline Hs.444212 & 0.8 & VSNL1 & Visinin-like 1 \\
\hline Hs.371222 & 0.8 & TMEM110 & Transmembrane protein 110 \\
\hline
\end{tabular}

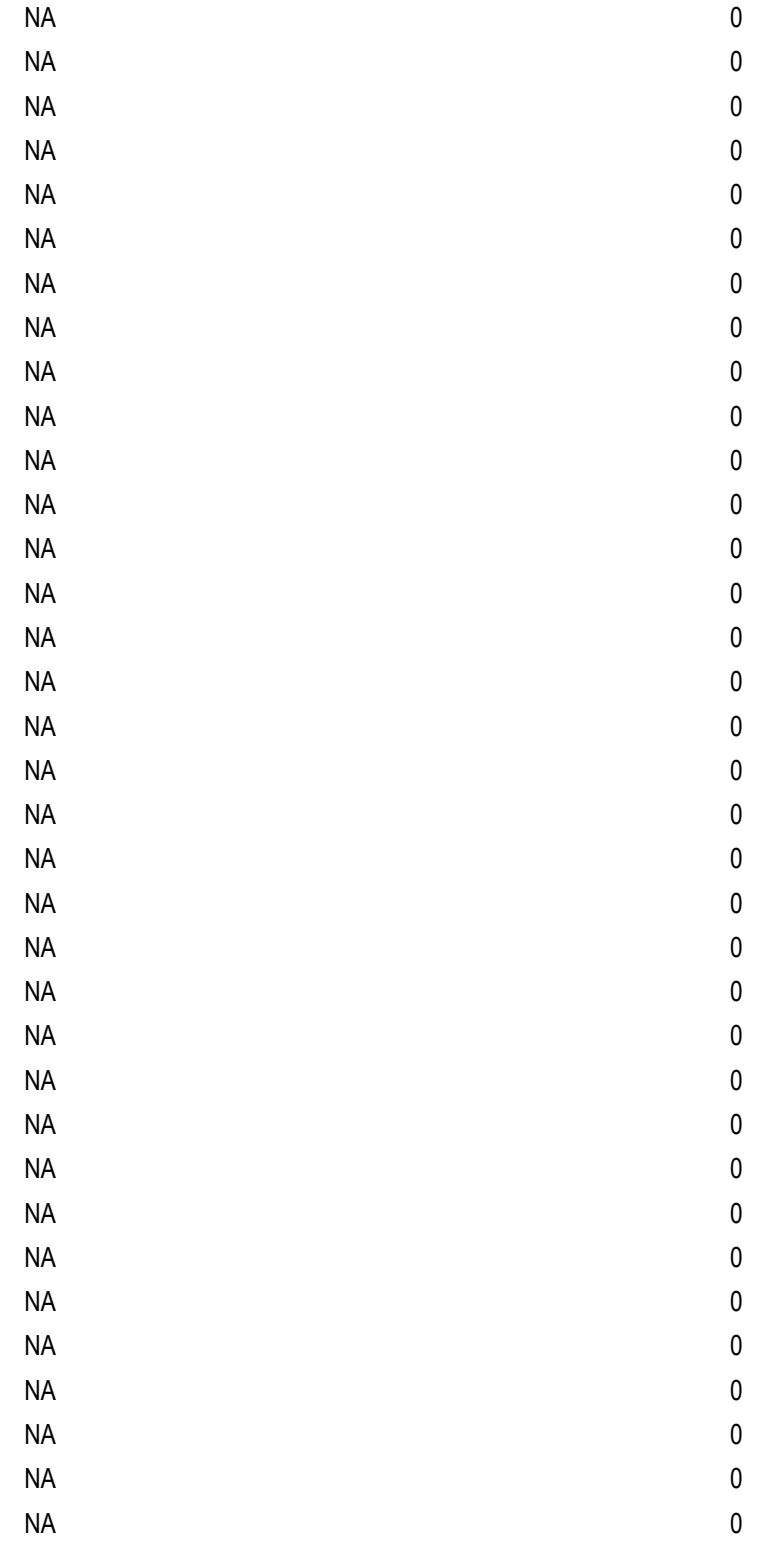




\begin{tabular}{|c|c|c|c|c|c|}
\hline Hs.568509 & 0.8 & ARHGEF2 & Rho/rac guanine nucleotide exchange factor (GEF) 2 & NA & 0 \\
\hline Hs. 475125 & 0.8 & ATXN10 & Ataxin 10 & NA & 0 \\
\hline Hs.145269 & 0.8 & TNFRSF10C & Tumor necrosis factor receptor superfamily, member $10 \mathrm{c}$, decoy without an intracellular domain & NA & 0 \\
\hline Hs. 637251 & 0.8 & & Transcribed locus & NA & 0 \\
\hline Hs. 632200 & 0.8 & THAP11 & THAP domain containing 11 & NA & 0 \\
\hline Hs.598124 & 0.7 & & Transcribed locus & NA & 0 \\
\hline Hs.370187 & 0.7 & STAU1 & Staufen, RNA binding protein, homolog 1 (Drosophila) & NA & 0 \\
\hline Hs. 632480 & 0.7 & DENND4B & DENN/MADD domain containing 4B & NA & 0 \\
\hline Hs. 468140 & 0.7 & FAM98A & Family with sequence similarity 98 , member $A$ & NA & 0 \\
\hline Hs.635459 & 0.7 & & Transcribed locus & NA & 0 \\
\hline Hs.116244 & 0.6 & WDR62 & WD repeat domain 62 & NA & 0 \\
\hline Hs.591761 & 0.6 & HOMER1 & Homer homolog 1 (Drosophila) & NA & 0 \\
\hline Hs.600886 & 0.5 & & Transcribed locus & NA & 0 \\
\hline Hs. 534770 & 0.4 & PKM2 & Pyruvate kinase, muscle & NA & 0 \\
\hline
\end{tabular}

Differentially expressed genes between Leigh patients with a SURF1 mutation and controls. Gene Ontology annotations are listed as specific as possible (highest level). FC: fold change, NA: not annotated. 
Supplementary table S3: Significantly enriched gene ontology biological process terms

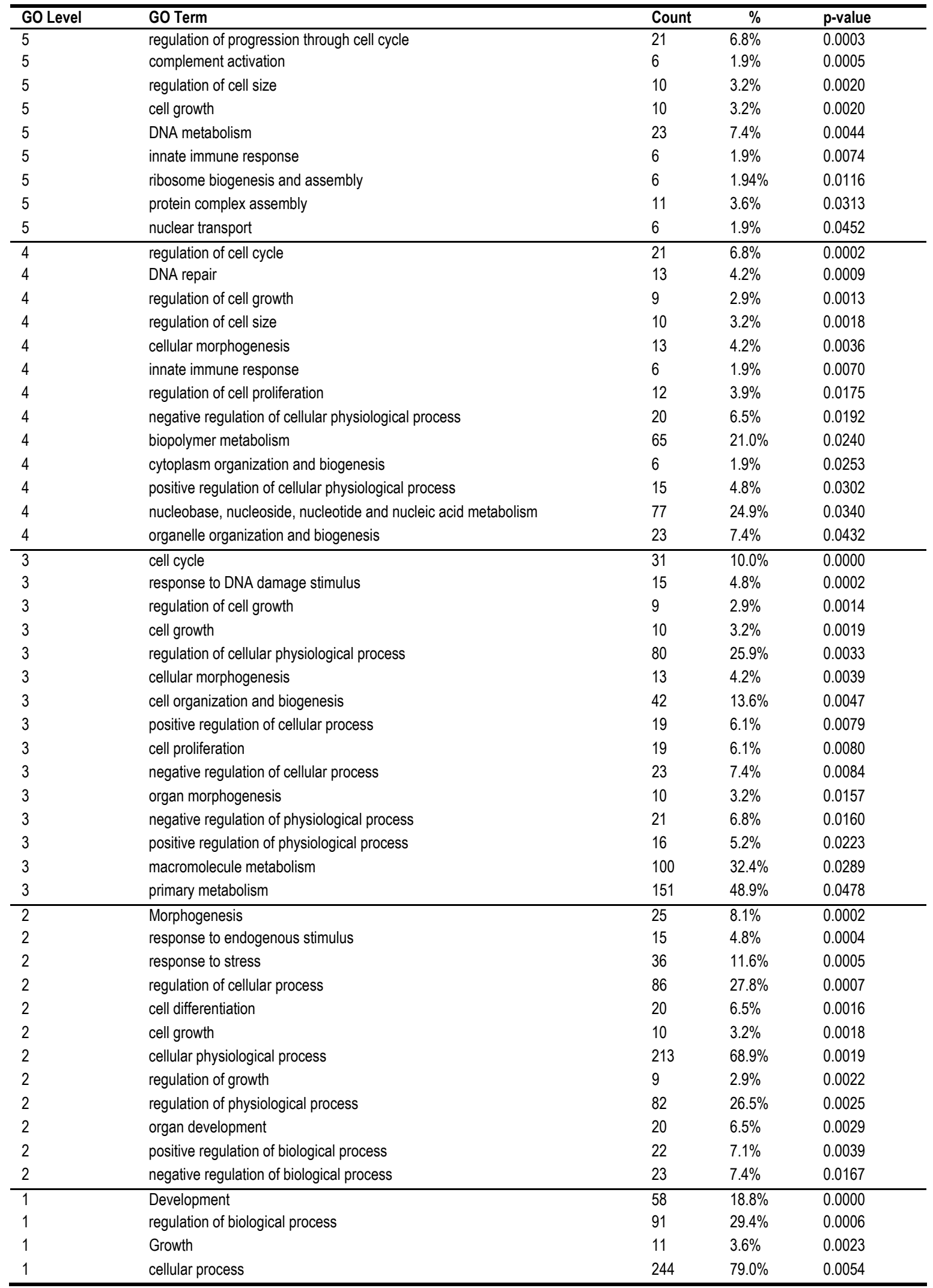

Significantly enriched gene ontology biological process terms $(p<0.05)$ in the list of differentially expressed genes, as determined by DAVID. 



\section{CHAPTER 4}

\section{Termination of damaged protein repair defines the occurrence of symptoms in carriers of the m.3243A>G tRNA ${ }^{\text {Leu }}$ mutation}

R.G.E. van Eijsden ${ }^{1,2,3 \$}{ }^{3}$, L.M.T. Eijssen ${ }^{4,5 \$}$, P.J. Lindsey ${ }^{4}$, C.M.M. van den Burg ${ }^{4}$, L.E. A. de Wit ${ }^{6}$, M.E. Rubio-Gozalbo ${ }^{7}$, C.E.M. de Die ${ }^{8}$, T. Ayoubi ${ }^{4,9}$, W. Sluiter ${ }^{6}$, I.F.M. de Coo ${ }^{10}$, H.J.M. Smeets ${ }^{1,2,4}$

${ }^{1}$ Department of Genetics and Cell Biology - Clinical Genetics, Maastricht University, Maastricht The Netherlands.

${ }^{2}$ Research Institute Growth \& Development, Maastricht University, Maastricht - The Netherlands. ${ }^{3}$ MicroArray Facility, VIB, Leuven - Belgium.

${ }^{4}$ Department of Population Genetics, Genomics \& Bioinformatics, Maastricht University, Maastricht - The Netherlands.

${ }^{5}$ BiGCaT Bioinformatics, Maastricht University, Maastricht - The Netherlands.

${ }^{6}$ Department of Biochemistry, Mitochondrial Research Unit, Erasmus MC, Rotterdam - The Netherlands.

${ }^{7}$ Department of Paediatrics and Laboratory Genetic Metabolic Diseases, Maastricht University Hospital, Maastricht - The Netherlands.

${ }^{8}$ Department of Clinical Genetics, Maastricht University Hospital, Maastricht - The Netherlands.

${ }^{9}$ Cardiovascular Research Institute Maastricht (CARIM), Maastricht University - Maastricht - The Netherlands.

${ }^{10}$ Department of Neurology, Erasmus MC, Rotterdam - The Netherlands.

${ }^{\$}$ Current affiliation.

Journal of Medical Genetics, in press 


\section{Abstract}

The m.3243A>G mutation in the mitochondrial tRNA ${ }^{\text {Leu(UUR) }}$ gene is an example of a mutation causing a very heterogeneous phenotype. It is the most frequent cause (80\%) of the MELAS syndrome (Mitochondrial Myopathy, Encephalopathy, Lactic Acidosis and Stroke-like episodes), but it can also lead in addition or separately to type 2 diabetes, deafness, renal tubulopathy and/or cardiomyopathy. To identify pathogenic processes induced by this mutation, we compared global gene expression levels of muscle biopsies from affected and unaffected mutation carriers with controls. Gene expression changes were relatively subtle. In the a-symptomatic group 200 transcripts were up- and 12 were down-regulated, whereas in the symptomatic group this was 15 and 52 respectively. In the a-symptomatic group, oxidative phosphorylation (OXPHOS) complex I and IV genes were induced. Protein turnover and apoptosis were elevated, most likely due to the formation of dysfunctional and reactive oxygen species (ROS) damaged proteins. These processes returned to normal in symptomatic patients. Components of the complement system were up-regulated in both groups, but the strongest in the symptomatic group, which might indicate muscle regeneration. Most likely, protein damage and OXPHOS dysfunction stimulate repair (protein regeneration) and metabolic adaptation (OXPHOS). In a-symptomatic individuals these processes suffice to prevent the occurrence of symptoms. However, in affected individuals the repair process terminates, presumably because of excessive damage, and switches to muscle regeneration, as indicated by a stronger complement activation. This switch leaves increasingly damaged tissue in place and muscle pathology becomes manifest. Therefore, the expression of complement components might be a marker for the severity and progression of MELAS clinical course.

\section{Introduction}

Mitochondrial encephalomyopathies due to defects in oxidative phosphorylation (OXPHOS) are genetically and clinically heterogeneous disorders. Different mutations in different genes can lead to the same disease manifestation, whereas one single mutation can result into a variety of clinical symptoms. Approximately $25 \%$ of the paediatric cases of OXPHOS disease is caused by a mutation in the mtDNA [23]. An example of a clinically heterogeneous mutation is the m.3243A>C point mutation in the mitochondrial tRNA ${ }^{\text {Leu(UUR) }}$ gene. This is the most frequent ( $80 \%$ ) cause of MELAS syndrome (Mitochondrial Myopathy, Encephalopathy, Lactic Acidosis and Stroke-like episodes, OMIM \# 540000), but can also lead in addition or separately to type 2 diabetes, deafness, renal tubulopathy and/or cardiomyopathy. Differences in mutation load can only partly explain the differences in disease severity and symptoms, but effects are not consistent and it is not the only factor involved [99]. A variety of studies have been performed to investigate possible correlations between mtDNA haplo groups or polymorphisms and clinical phenotypes, or to study clinical and pathophysiological features among individuals carrying the m.3243A>C 
mutation [100-105]. However, an explanation for the differences in threshold for clinical expression and for the clinical heterogeneity has not yet been found.

Therefore, we applied gene expression profiling as an unbiased approach to identify the molecular processes which are induced by the m.3243A>G mutation in different individuals and lead to the development and progression of clinical symptoms. Microarray gene expression experiments were carried out on skeletal muscle from subjects carrying the m.3243A>G mutation and controls. Gene expression differences were compared between mutation carriers with and without symptoms to characterise the pathogenic processes involved.

\section{Materials \& Methods}

Patients and controls

For gene expression profiling, needle biopsies from the quadriceps muscle were obtained from seven subjects carrying the MELAS m.3243A>C mutation (two men and five women, age at diagnosis 32 to 70 years, table 1 ) and six controls (two men and four women, age at muscle biopsy between 17 and 64 years). After biopsy, the muscle samples were immediately frozen in liquid nitrogen. The mutation carriers (Mut3243 group) were subdivided into two groups: one group ( $n=4$, three women and one man) having the classical clinical MELAS symptoms which is the symptomatic group ( 3243 group) and a second group ( $n=3$, two women and one man) without clinical symptoms, the a-symptomatic group (AS3243 group). For enzyme histochemistry to detect the expression of superoxide-producing enzymes (see below), muscle cryosections were used from seven MELAS m.3243A>G mutation carriers (three symptomatic and four a-symptomatic, table 1) of which two were also used for the gene expression experiment, and nine additional controls (age at muscle biopsy between 2 and 55 years). For the oxprot assay (see below), muscle biopsies were used from 11 MELAS m.3243A>C mutation carriers (eight symptomatic and three a-symptomatic, table 1), of which five were also used for the microarray experiment, and nine controls of which three were also used in the microarray experiment (age at muscle biopsy between 2 and 53 years). 
Table 1: Mutation carriers

\begin{tabular}{llllllll}
\hline Subject ID & $\begin{array}{l}\text { m.3243A }>\text { G skeletal muscle } \\
\text { heteroplasmy level }\end{array}$ & (A-)Symptomatic & Sex & Age at diagnosis & $\begin{array}{l}\text { Microarray } \\
\text { experiment }\end{array}$ & Oxprot assay & DHE staining \\
\hline 5 & $50 \%$ & S & F & 52 & + & + & - \\
12 & $53 \%$ & S & F & 36 & + & + & - \\
13 & $16 \%$ & AS & F & 70 & + & - & - \\
17 & $32 \%$ & S & M & 44 & + & - & + \\
24 & $73 \%$ & S & F & 33 & + & + & + \\
25 & $60 \%$ & AS & M & 32 & + & + & + \\
26 & $15 \%$ & AS & F & 49 & - & + & + \\
27 & $28 \%$ & AS & F & 15 & - & - & + \\
28 & $28 \%$ & AS & F & 23 & - & - & + \\
29 & $63 \% *$ & S & M & 25 & - & - & + \\
30 & $65 \%$ & AS & F & 69 & + & + & - \\
37 & $56 \%$ & S & M & 46 & - & + \\
38 & $92 \%$ & S & M & 6 & - & + \\
41 & $82 \%$ & S & M & 28 & - & + \\
45 & $64 \%$ & S & M & 49 & - & + \\
46 & $75 \%$ & S & F & 37 & - & + \\
\hline
\end{tabular}

Overview of m.3243A>G mutation carriers used for the microarray gene expression experiment, oxprot assay, and DHE staining.

* Mutation percentage in fibroblast cells.

F: Female, M: Male, S: with clinical symptoms (symptomatic), AS: without clinical symptoms (a-symptomatic).

\section{m.3243A>G mutation detection}

Forward 5'-CAACTTAGTATTATACCCACAC-3' and reverse primers 5'-GATTACGAATGGCTTGCTTT-3' were used to amplify the fragment carrying the m.3243A>C mutation by polymerase chain reaction (PCR) using a FAM-labelled primer in the last PCR cycle. Heteroplasmy levels were determined by mutation specific restriction digestion with the Hawaii enzyme resulting in a wildtype fragment of 128 bases and a mutant fragment of 56 bases long. Digested products were analyzed on an ABI-PRISM 3100 genetic analyzer using the GeneScan Analysis 3.7 software package (Applied Biosystems, Foster City, CA, USA). The ratio of the area under the mutant peak and the sum of the areas under the mutant and wild-type peaks were calculated to determine the heteroplasmy levels of the m.3243A>G mutation.

\section{Microarray procedure}

Total RNA was isolated using the TRIzol reagent (Invitrogen, Carlsbad, CA, USA) and purified with the RNeasy clean-up kit (Qiagen, Hilden, Germany). RNA quantity and purity were determined spectrophotometrically using the Nanodrop ND-1000 (Nanodrop Technologies, Wilmington, DE, USA) and RNA integrity was assessed by determining the RNA 28S/18S ratio using the Bioanalyser 2100 (Agilent Technologies, Santa Clara, CA, USA). Muscle RNA spiked with four bacterial RNA transcripts was reverse transcribed into CDNA and amplified in a two-round amplification reaction according to the manufacturer's protocol (Affymetrix, Santa Clara, CA, USA). A mixture of cDNA and added hybridisation controls was hybridised on Affymetrix HG-U95Av2 chips, followed by staining and washing steps in the GeneChip fluidics station 400 (Affymetrix, Santa Clara, CA, USA) 
according to the manufacturer's procedures. To assess the raw probe signal intensities, chips were scanned using the GeneChip scanner 3000 (Affymetrix, Santa Clara, CA, USA).

\section{Microarray data analysis and data mining}

$\mathrm{R}$, a free software environment for statistical computing and graphics, was used in combination with the gnlm and the affy libraries to perform the data preparation and analysis [40, 68-70]. Version 8 of the Unigene-based probe set definition files (CDF files) as described by Dai et al. was used to combine the individual probes into probe sets [71]. These CDF files are based on Unigene build \#192. The expresso method of the affy library, without background correction, with constant normalisation, use of perfect match signal intensities only, and the "mas" summarisation method were used to calculate the probeset intensities from the individual probe signals.

Normal linear regression models, implemented by the gnlr function within the gnlm library, were used to asses differentially expressed genes between m.3243A>C subjects and controls. For the patient versus control comparisons, two models were fitted. In one model all mutation carriers (Mut3243 group) were compared to the controls, to select the genes which have an effect due the presence of the mutation, irrespective of the presence of clinical symptoms or not. A second model was fitted to select the genes with a significant different expression for the S3243 or the AS3243 group compared to the controls, but also with a significant difference in expression between the S3243 and the AS3243 group. Differences due to experimental variation were corrected for by including the data from the RNA spikes and hybridisation controls as covariates in the models. Because hybridisations were carried out on two days, the hybridisation days were also taken into account in the modelling procedure. The Akaike information criterion (AIC), representing the likeliness of each model penalised for the complexity of the model, was used to identify the best fitting models and genes with a significant change in expression between two groups [72]. The AIC was adjusted to be comparable to a two-sided chi-squared test with a significance level of $99.9 \%$ ( $p$-value<0.001), making the analysis more stringent [73]. The webbased Database for Annotation, Visualisation and Integrated Discovery (DAVID) was used to assess enriched gene ontology terms within the gene lists produced by the microarray data analysis [76].

\section{Quantitative Real-time PCR}

Primers were designed using Primer Express ${ }^{\circledR}$ software version 3.0 (Applied Biosystems, Foster City, CA, USA). CDNA was prepared from $2 \mu \mathrm{g}$ of total RNA in a standard reverse transcriptase reaction. PCR was performed in a 7700 Realtime PCR System (Applied Biosystems, Foster City, CA, USA) and a BioRad MyiQ Single-Colour Real-Time PCR Detection System (Bio-Rad, Hercules, CA,

USA) using Eurogentec $\mathrm{qPCR}^{\mathrm{TM}}$ Mastermix Plus for SYBR Green ${ }^{\circledR}$ I. Cycling conditions were: an initial step of 2 minutes at $50^{\circ} \mathrm{C}$, activation of the Hot Goldstar enzyme at $95^{\circ} \mathrm{C}$ for 10 minutes, and 40 cycles of 15 seconds at $95^{\circ} \mathrm{C}$ followed by 1 minute at $60^{\circ} \mathrm{C}$ (denaturation, annealing, and 
elongation). The ADP-ribosylation factor 1 (ARF1) gene was used as an internal reference because of its constant expression level in muscle [77]. Genes for which QRT-PCR was performed were complement component $1, r$ subcomponent $\left(C_{1} R\right)$, complement component, s subcomponent (C1S), complement component 3 (C3), and clusterin (CLU). Primers used for amplification are listed in table 2. Data was analyzed in R using the elliptic function of the growth library to implement normal linear regression models $[69,70]$. The AIC was adjusted to be comparable to a two-sided chi-squared test with a significance level of $95 \%$, making the analysis more stringent [73].

Table 2: QRT-PCR primers

\begin{tabular}{llll}
\hline Gene symbol & Description & Forward primer (5'-3') & Reverse primer (5'-3') \\
\hline ARF1 & ADP-ribosylation factor 1 & AATCAGCTCCGGAACCAGAA & GCAGAGGGCAAGAGGAGGA \\
C1R & Complement component 1, r subcomponent & TCTGTGCTGGACACCCATCTC & GCAAAAACGCCCCCACTAT \\
C1S & Complement component 1, s subcomponent & TGGATAATGAAGACTATGCAGGAAAA & GGAGAGGCTGGTGGGATGTA \\
C3 & Complement component 3 & TGGCCATTGAGCAGACCAT & CGTGCGCTGCTGTCCA \\
CLU & Clusterin & GCTGCAGGAATACCGCAAA & CCGTAGGTGCAAAAGCAACA \\
\hline
\end{tabular}

Primer sequences of the primers used for quantitative real-time PCR validation of microarray gene expression data.

\section{Detection of superoxide-generating enzymes in muscle}

The expression of superoxide radical-producing enzymes in muscle was quantified by enzyme histochemistry of skeletal muscle cryosections from seven MELAS m.3243A>G mutation carriers (three symptomatic and four a-symptomatic, table 1) of which two were also used for the gene expression experiment and nine additional controls (age at muscle biopsy between 2 and 55 years) using dihydroethidium (DHE) as a probe for superoxide. Ten- $\mu \mathrm{m}$ thick cryosections were stored at $-80^{\circ} \mathrm{C}$. After thawing the sections, they were stained by $5 \mu \mathrm{M}$ dihydroethidium and 0.5 $\mu \mathrm{g} / \mathrm{mL}$ Hoechst 33258 in phosphate-buffered saline (PBS) for $30 \mathrm{~min}$ at $37^{\circ} \mathrm{C}$ in a humidified atmosphere as described previously [106]. The orange fluorescence of the superoxide-specific reaction product of DHE oxidation [79] was measured using a fluorescence inverted microscope (Olympus IX50) equipped with a 460-490 nm band pass excitation filter and 515-nm emission IFbarrier filter, digitised with a F-view camera (Soft Imaging System), and analyzed offline (AnalySIS 3.1; Soft Imaging System, Münster, Germany). The signal was expressed in arbitrary units reflecting the total fluorescence intensity of all events divided by the number of blue fluorescent nuclei. At least 300 nuclei per section were counted in two to three consecutive sections. Onesided and two-sided t-tests on the averages of the replicate measurements were used to assess statistical differences between the m.3243A $>C$ mutation carriers in general and the control groups, and between the AS3243 and S3243 group respectively.

\section{Oxidative protein damage}

Oxidative damage to proteins in the muscle biopsies was determined by quantifying the amount of carbonyl groups per mg protein using an ELISA assay optimised for small amounts of proteins (Oxprot assay). For this assay, $10 \mathrm{mg}$ wet weight (ww) of the skeletal muscle biopsies was used 
from nine controls (age at muscle biopsy between 2 and 53 years), of which three were also used in the microarray experiment. Additionally, skeletal muscle biopsies were used from 11 MELAS m.3243A>C mutation carriers (eight symptomatic and three a-symptomatic, table 1), of which five were also used for the microarray experiment. The snap-frozen biopsies were pulverised in the presence of $19 \mathrm{~mL} / \mathrm{g} w \mathrm{w}$ of RIPA buffer $(50 \mathrm{mM}$ Tris-HCl, $150 \mathrm{mM} \mathrm{NaCl}, 1 \mathrm{mM}$ EDTA, pH 7.4, containing $0.25 \%$ sodium deoxycholate, $1 \%$ NP40 and supplemented with a protease inhibitor mixture (PIM) of one Complete tablet per $50 \mathrm{~mL}$ (Roche Diagnostics, Almere, The Netherlands), 1 mM 4-(2-aminoethyl)-benzenesulfonyl-fluoride hydrochloride (Pefabloc SC, Roche) and $2 \mathrm{mM}$ diisopropyl fluorophosphate (DFP) (Fluka Chemica, Steinheim, Switzerland) in a small Bessman tissue pulveriser (Fisher Scientific) precooled with liquid nitrogen. For carbonyl determination the tissue homogenate was first incubated on ice for $30 \mathrm{~min}$, and then centrifuged at 16,000 $\mathrm{g}$ for 20 min at $4^{\circ} \mathrm{C}$, and the supernatant was diluted in PBS to $0.5 \mu \mathrm{g} / \mathrm{mL}$ protein. Next $200 \mu \mathrm{l}$ was transferred into triplicate wells of a Nunc Maxisorp microplate. The amount of carbonyl groups resulting from protein oxidation were determined as described by Alamdari et al .[78]. A technical replicate was performed for seven out of nine patient samples and for three out of seven control samples. A one-sided t-test on the averages of the replicate measurements was used to assess statistical differences between the patient and the control groups.

\section{Results}

Gene expression profiling of symptomatic and a-symptomatic m.3243A $>G$ mutation carriers

Gene expression levels were measured for 7084 transcripts in each sample. The expression of 407 transcripts was significantly different between one of the patient groups and the control group (supplementary table 1 and table 3). For 128 transcripts, the expression level differed significantly between all mutation carriers (Mut3243), irrespective of the presence of clinical symptoms, and controls. The majority (112 out of 128 or $87.5 \%$ ) of these transcripts was up-regulated and 16 (12.5\%) transcripts were down-regulated. The expression level of 212 transcripts differed between the a-symptomatic group (AS3243) and the controls. The vast majority ( $94.3 \%$ or 200 transcripts) was up-regulated and only 5.7\% (12 transcripts) was down-regulated. A total of 67 (15 up- and 52 downregulated) transcripts was significantly different between the symptomatic group (S3243) and the controls. Gene expression differences were in general small. For the Mut3243 versus controls comparison there were 18 up-regulated genes and no down-regulated genes with a fold-change larger than 1.5. In the AS3243 versus controls comparison, none of the down-regulated genes and seven of the up-regulated genes had a fold change larger than 1.5. In the S3243 versus controls comparison, only one of the down-regulated genes and four of the up-regulated genes had a foldchange larger than 1.5 .

Because of the subtle gene expression differences, a process based analysis was carried out. DAVID functional annotation clustering was performed on the differentially expressed genes at 
the GO biological process level 5. For the Mut3243 versus controls comparison, processes related to protein transport, programmed cell death, and the immune system, more specifically the complement system, were identified as functional gene clusters (table 4). In addition to the complement related genes identified on the microarray, a number of other complement components were shown to be differentially expressed by QRT-PCR (see below). The gene expression changes pointed to stimulation of the complement system, stimulation of protein transport, and both stimulation and inhibition of programmed cell death. Upon further analysis, the stimulation of the protein transport process in fact more closely resembled a stimulation of protein synthesis. Three out of the eight genes are related to the ribosome or the endoplasmatic reticulum (ER). The ribosomal protein L23 (RPL23), which is 1.5-fold up-regulated in the Mut3243 group compared to the controls, is a component of the 605 ribosomal subunit and is involved in protein synthesis. The gene coding for the translocation associated membrane protein 2 (TRAM2) was 1.4-fold up-regulated in Mut3243 group compared to controls. The TRAM2 protein is involved in the posttranslational processing of secretory and membrane proteins at the ER membrane [107]. The ribosome binding protein 1 homolog (RRBP1) gene is 1.2-fold up-regulated in the Mut3243 group compared to the controls. It mediates the interaction between the ribosome and the ER and has an mRNA stabilizing function in yeast [108]. Programmed cell death appeared to be both stimulated and inhibited in the Mut3243 group compared to controls. Pro-apoptotic processes are the 1.2 fold up-regulation of the death-associated protein (DAP), a stimulator of a variety of cell death systems $[109,110]$, the 1.2 fold up-regulation of p8 protein, also known as COM1, which is a cell cycle regulator [111] and the 0.7 fold down-regulation of the testis enhanced gene transcript (TEGT), which is also known as the inhibitor of the pro-apoptotic BAX protein (BI1). Apoptosis inhibiting effects are the 1.7 fold up-regulation of annexin $A_{1}$ (ANXA1), also known as a p35 gene, which inhibits caspase $[112,113]$ and the 1.1 fold up-regulation of histone deacetylase 1 (HDAC1). HDAC1 inhibitors have been used in cancer treatment due to their pro-apoptotic effect [114]. Finally, the 2.1 fold up-regulation of clusterin (CLU) is more ambiguous as CLU has been related to both stimulation and inhibition of programmed cell death [115]. For the AS3243 versus controls comparison, four functional gene clusters were identified. The gene clusters were involved in oxidative energy metabolism, programmed cell death, protein synthesis, and intracellular protein trafficking (table 5). According to the direction of the gene expression level changes, each of these processes was up-regulated. Changes in oxidative energy metabolism included the up-regulation of the genes coding for three subunits of the NADH dedydrogenase complex (complex I of the OXPHOS system): NDUFB8 (1.4-fold), NDUFC1 (1.2-fold), and NDUFS3 (1.3fold). Moreover, one subunit of the cytochrome-c-oxidase (COX) complex (complex IV of the OXPHOS system), COX4l1, was 1.2-fold up-regulated. Induction of protein synthesis is illustrated by the up-regulation of a number translation initiation factors, EIF2AK1 (1.2-fold), EIF2B4 (1.3-fold), and EIF2S1 (1.4-fold). Furthermore, a subunit of the ribosomal $40 S$ complex (RPS21) and the mitochondrial ribosomal protein L23 (MRPL23) were 1.5-fold and 1.3-fold up-regulated respectively. The 1.1 fold up-regulation of the p53 gene, the 1.2 fold up-regulation of homeodomain interacting protein kinase 2 (HIPK2), which phosphorylates the p53 genes and thereby induces apoptosis 
[116], and the 1.1 fold up-regulation of serine/threonine kinase 17a (STK17A or DRAK1), which has a catalytic domain related to that of death-associated protein kinase and can induce the morphological changes of apoptosis in NIH $3 \mathrm{~T}_{3}$ cells [117], point to a stimulation of apoptosis in a-symptomatic m.3243A $>\mathrm{G}$ mutation carriers compared to controls. No functional gene clusters were identified for the $\mathrm{S} 3243$ versus controls comparison.

Based on previous microarray results and observations in Leigh syndrome patients with a mutation in the SURF1 gene (unpublished results) we investigated the gene expression changes of a number of genes of the complement system by QRT-PCR (table 6). Although the microarray analysis indicated that the complement component $C_{1} R$ was differentially expressed in mutation carriers in general compared to controls, QRT-PCR analysis showed a significantly stronger upregulation of $C 1 R$ in the $S 3243$ group (6.8-fold) compared to the AS3243 group (2.7-fold) when compared to controls. Although the data for complement component C1S was not significant by microarray analysis, QRT-PCR analysis indicated that this transcript was significantly up-regulated in the Mut3243 group compared to the control group. QRT-PCR analysis of complement component $\mathrm{C}_{3}$, which was not present in the microarray dataset, indicated a significant 4.1-fold up-regulation in the Mut3243 group compared to controls. The up-regulation of clusterin was also confirmed by QRT-PCR, indicating a 4.3-fold up-regulation in the Mut3243 group compared to controls. There was a good correspondence between the microarray and QRT-PCR results, which is in general the experience with Affymetrix microarray results. 
Table 3: Overview of the gene expression changes comparing mutation carriers with or without clinical symptoms to the control group

\begin{tabular}{llllll}
\hline Comparison & UP & & DOWN & & TOTAL \\
\hline S3243 versus controls & 15 & $56.7 \%$ & 52 & $43.3 \%$ & 67 \\
FC $>1.5$ & 4 & $3.3 \%$ & 1 & $0.8 \%$ & \\
Max. FC & 2.2 & & 1.7 & & \\
\hline AS3243 versus controls & 200 & $94.3 \%$ & 12 & $5.7 \%$ & 212 \\
FC $>1.5$ & 7 & $3.3 \%$ & 0 & $0.0 \%$ & \\
Max. FC & 4.9 & & 1.4 & & 128 \\
\hline Mut3243 versus controls & 112 & $87.5 \%$ & 16 & $12.5 \%$ & $0.0 \%$ \\
FC $>1.5$ & 18 & $14.1 \%$ & 0 & & \\
Max. FC & 2.7 & & 1.5 & & \\
\hline
\end{tabular}

For the symptomatic group 15 (56.7\%) transcripts were up-regulated and 52 (43.3\%) were down-regulated. In the a-symptomatic group 200 (94.3\%) transcripts were up-regulated and 12 (5.7\%) were down-regulated. For the mutation carriers in general, 112 ( $87.5 \%)$ transcripts were up-regulated and 16 (12.5\%) were down-regulated.

S3243: m.3243A>G mutation carriers with clinical symptoms. AS3243: m.3243A>G mutation carriers without clinical symptoms. Mut3243: m.3243A>G mutation carriers in general, irrespective of the presence of clinical symptoms. FC: fold change. Max. FC: Maximum fold change.

Oxidative protein damage and superoxide radicals in muscle of m.3243A $>G$ mutation carriers

Oxidative damage to muscle proteins determined by the amount of carbonyl groups was significantly increased in nine subjects carrying the m.3243A>C mutation at levels between $50-92 \%$ ( $p=0.03$ in a one-sided t-test) compared to controls. The amount of carbonyl groups was 2.06 (SD: 0.86 ) and 1.34 (SD: 0.65) nmol per mg protein for the mutation carriers and controls respectively, illustrating a significant 1.5 -fold increase. Although there was no significant difference in the amount of carbonyl groups between the S3243 and the AS3243 group, the detected amount of carbonyl groups per mg protein for one of the a-symptomatic mutation carriers was much less than the amount in the other two a-symptomatic mutation carriers ( 0.27 compared to 2.04 and $3.29 \mathrm{ng}$ carbonyl/mg protein respectively). The expression of superoxide-production enzymes was significantly increased in muscle of m.3243A $>\mathrm{G}$ mutation carriers. As shown in figure 1 , the staining intensity for these enzymes in muscle cryosections of five muscle biopsies from m.3243A>C mutation carriers (mutation loads between 15-73\%) was significantly ( $p=0.001$ in a onesided t-test) 3.8-fold higher compared to control samples. There were no significant differences in the expression of superoxide-producing enzymes between symptomatic and a-symptomatic mutation carriers compared to controls according to a two-sided t-test. 
Table 4: David functional annotation clustering results at the level of GO biological process level 5 for the Mut3243 versus control comparison

\begin{tabular}{lllll}
\hline \multicolumn{1}{l}{ Functional Group 1: Immune response } & & & \\
\hline G0 terms & UG ID & Symbol & FC & Genes \\
\hline complement activation & Hs.169998 & BST1 & 1.2 & bone marrow stromal cell antigen 1 \\
innate immune response & Hs.436657 & CLU & 2.1 & clusterin \\
humoral immune response & Hs.155597 & CFD & 1.7 & complement factor d (adipsin) \\
& Hs.384598 & SERPING1 & 1.9 & serpin peptidase inhibitor, clade g (c1 inhibitor), member 1,
\end{tabular}

\begin{tabular}{lllll}
\hline Functional Group 2: Protein transport & & & & \\
\hline G0 terms & UG ID & Symbol & FC & Genes \\
\hline intracellular protein transport & Hs.507755 & DCAMKL1 & 1.4 & doublecortin and cam kinase-like 1 \\
protein transport & Hs.527919 & KPNA3 & 0.7 & karyopherin alpha 3 (importin alpha 4) \\
intracellular transport & Hs.444767 & KIF13B & 1.3 & kinesin family member 13b \\
establishment of cellular localization & Hs.29282 & MAP3K3 & 0.9 & mitogen-activated protein kinase kinase kinase 3 \\
& Hs.529044 & RAB22A & 1.2 & rab22a, member ras oncogene family \\
& Hs.516790 & ARHGEF2 & 1.2 & rho/rac guanine nucleotide exchange factor (gef) 2 \\
& Hs.406300 & RPL23 & 1.5 & ribosomal protein I23 \\
& Hs.472213 & RRBP1 & 1.2 & ribosome binding protein 1 homolog 180kda (dog) \\
& Hs.520182 & TRAM2 & 1.4 & translocation associated membrane protein 2 \\
\hline Functional Group 3: Programmed cell death & & & & \\
\hline G0 terms & & & & \\
\hline regulation of apoptosis & UG ID & Symbol & FC & Genes \\
regulation of programmed cell death & Hs.494173 & ANXA1 & 1.7 & annexin a1 \\
negative regulation of programmed cell death & Hs.436657 & CLU & 2.1 & clusterin \\
apoptosis & Hs.75189 & DAP & 1.2 & death-associated protein \\
& Hs.88556 & HDAC1 & 1.1 & histone deacetylase 1 \\
& Hs.513463 & P8 & 1.2 & p8 protein (candidate of metastasis 1) \\
& Hs.35052 & TEGT & 0.7 & testis enhanced gene transcript (bax inhibitor 1) \\
\hline
\end{tabular}

Three functional gene clusters could be identified which contained genes related to immune response, protein transport, and programmed cell death.

GO: Gene ontology. UG ID: Unigene cluster ID. FC: fold change.

Table 5: David functional annotation clustering results at the level of GO biological process level 5 for the AS3243 versus control comparison

\begin{tabular}{lllll}
\hline \multicolumn{1}{l}{ Functional Group 1: Oxidative energy metabolism } & & & \\
\hline G0 terms & UG ID & Symbol & FC & Gene Title \\
\hline $\begin{array}{llll}\text { oxidative phosphorylation } \\
\text { ATP synthesis coupled electron transport }\end{array}$ & Hs.295917 & ATP6V1B2 & 1.3 & atpase, h+ transporting, lysosomal 56/58kda, v1 subunit b2 \\
electron transport & Hs.69745 & FDXR & 1.4 & ferredoxin reductase \\
& Hs.445350 & FMO3 & 1.3 & flavin containing monooxygenase 3 \\
& Hs.523215 & NDUFB8 & 1.4 & nadh dehydrogenase (ubiquinone) 1 \\
& Hs.84549 & NDUFC1 & 1.2 & nadh dehydrogenase (ubiquinone) 1 \\
& & & & subcomplex unknown, 1, 6kda \\
& Hs.502528 & NDUFS3 & 1.3 & nadh dehydrogenase (ubiquinone) \\
& & & fe-s protein 3, 30kda (nadh-coenzyme q reductase)
\end{tabular}




\begin{tabular}{|c|c|c|c|c|}
\hline \multicolumn{5}{|l|}{ Functional Group 2: Programmed cell death } \\
\hline GO terms & UG ID & Symbol & FC & Genes \\
\hline positive regulation of programmed cell death & Hs.264482 & ATG12 & 1.2 & atg12 autophagy related 12 homolog (s. cerevisiae) \\
\hline apoptosis & Hs. 517517 & EP300 & 1.1 & e1a binding protein p300 \\
\hline regulation of apoptosis & Hs.487294 & ERCC2 & 1.1 & $\begin{array}{l}\text { excision repair cross-complementing rodent repair deficiency, } \\
\text { complementation group } 2 \text { (xeroderma pigmentosum d) }\end{array}$ \\
\hline \multirow[t]{7}{*}{ regulation of programmed cell death } & Hs.397465 & HIPK2 & 1.2 & homeodomain interacting protein kinase 2 \\
\hline & Hs.87247 & HRK & 0.9 & harakiri, bcl2 interacting protein (contains only bh3 domain) \\
\hline & Hs. 139896 & MAEA & 1.4 & macrophage erythroblast attacher \\
\hline & Hs. 462590 & MY018A & 1.5 & myosin XVIIIA \\
\hline & Hs.2200 & PRF1 & 0.8 & perforin 1 (pore forming protein) \\
\hline & Hs.268887 & STK17A & 1.1 & serine/threonine kinase 17a (apoptosis-inducing) \\
\hline & Hs. 408312 & TP53 & 1.1 & tumor protein p53 (li-fraumeni syndrome) \\
\hline \multicolumn{5}{|l|}{ Functional Group 3: Protein synthesis } \\
\hline GO terms & UG ID & Symbol & FC & Genes \\
\hline regulation of protein biosynthesis & Hs.520205 & EIF2AK1 & 1.2 & eukaryotic translation initiation factor 2-alpha kinase 1 \\
\hline regulation of cellular biosynthesis & Hs. 169474 & EIF2B4 & 1.3 & $\begin{array}{l}\text { eukaryotic translation initiation factor } 2 \mathrm{~b} \text {, } \\
\text { subunit } 4 \text { delta, } 67 \mathrm{kda}\end{array}$ \\
\hline regulation of protein metabolism & Hs. 151777 & EIF2S1 & 1.4 & $\begin{array}{l}\text { eukaryotic translation initiation factor } 2 \text {, } \\
\text { subunit } 1 \text { alpha, } 35 \mathrm{kda}\end{array}$ \\
\hline \multirow[t]{5}{*}{ protein biosynthesis } & Hs.3254 & MRPL23 & 1.3 & mitochondrial ribosomal protein $\mathrm{I} 23$ \\
\hline & Hs.190968 & RPS21 & 1.5 & ribosomal protein s21 \\
\hline & Hs.404119, & TSTA3 & 1.2 & tissue specific transplantation antigen p35b \\
\hline & Hs.22616 & & 1.3 & kiaa0664 \\
\hline & Hs.3459 & & 1.4 & kiaa1970 protein \\
\hline \multicolumn{5}{|c|}{ Functional Group 4: Intracellular protein trafficking } \\
\hline GO terms & UG ID & Symbol & FC & Genes \\
\hline intracellular transport & Hs. 518460 & AP2M1 & 1.3 & adaptor-related protein complex 2 , mu 1 subunit \\
\hline establishment of cellular localization & Hs.12107 & CHMP2A & 1.4 & chromatin modifying protein $2 a$ \\
\hline intracellular protein transport & Hs. 546250 & DYNC112 & 1.1 & dynein, cytoplasmic 1 , intermediate chain 2 \\
\hline \multirow[t]{5}{*}{ protein transport } & Hs.87726 & GGA3 & 1.2 & $\begin{array}{l}\text { golgi associated, gamma adaptin ear containing, } \\
\text { arf binding protein } 3\end{array}$ \\
\hline & Hs. 12457 & NUP133 & 1.6 & nucleoporin 133kda \\
\hline & Hs.310645 & RAB1A & 1.2 & rab1a, member ras oncogene family \\
\hline & Hs. 180711 & STX3A & 1.2 & syntaxin 3a \\
\hline & Hs.408312 & TP53 & 1.1 & tumor protein p53 (li-fraumeni syndrome) \\
\hline
\end{tabular}

Four functional gene clusters could be identified which contained genes related to oxidative energy metabolism, programmed cell death, protein synthesis, and protein transport.

GO: Gene ontology. UG ID: Unigene cluster ID. FC: fold change. 
Table 6: Quantitative realtime PCR (QRT-PCR) results

\begin{tabular}{|lll|ll|ll|}
\cline { 4 - 7 } & & & \multicolumn{2}{l|}{ MicroArray $(\sim p<0.001)$} & \multicolumn{2}{l|}{ QRT-PCR $(\sim<<0.05)$} \\
\hline Symbol & Name & Process & Significant & FC & Significant & FC \\
\hline C1R & Complement component 1, r subcomponent & Complement & Mut3243 & 2.1 & AS3243 & $2.7 \uparrow$ \\
& & & & & S3243 & $6.8 \uparrow$ \\
C1S & Complement component 1, s subcomponent & Complement & NS & - & Mut3243 & $4.6 \uparrow$ \\
C3 & Complement component 3 & Complement & NA & NA & Mut3243 & $4.1 \uparrow$ \\
CLU & Clusterin & Complement & Mut3243 & $2.1 \uparrow$ & Mut3243 & $4.3 \uparrow$ \\
\hline
\end{tabular}

The "significant" column lists whether and for which group comparison the gene expression was significantly different. NS: Not significant, NA: Not in microarray dataset, FC: fold change, $\uparrow:$ up-regulated.

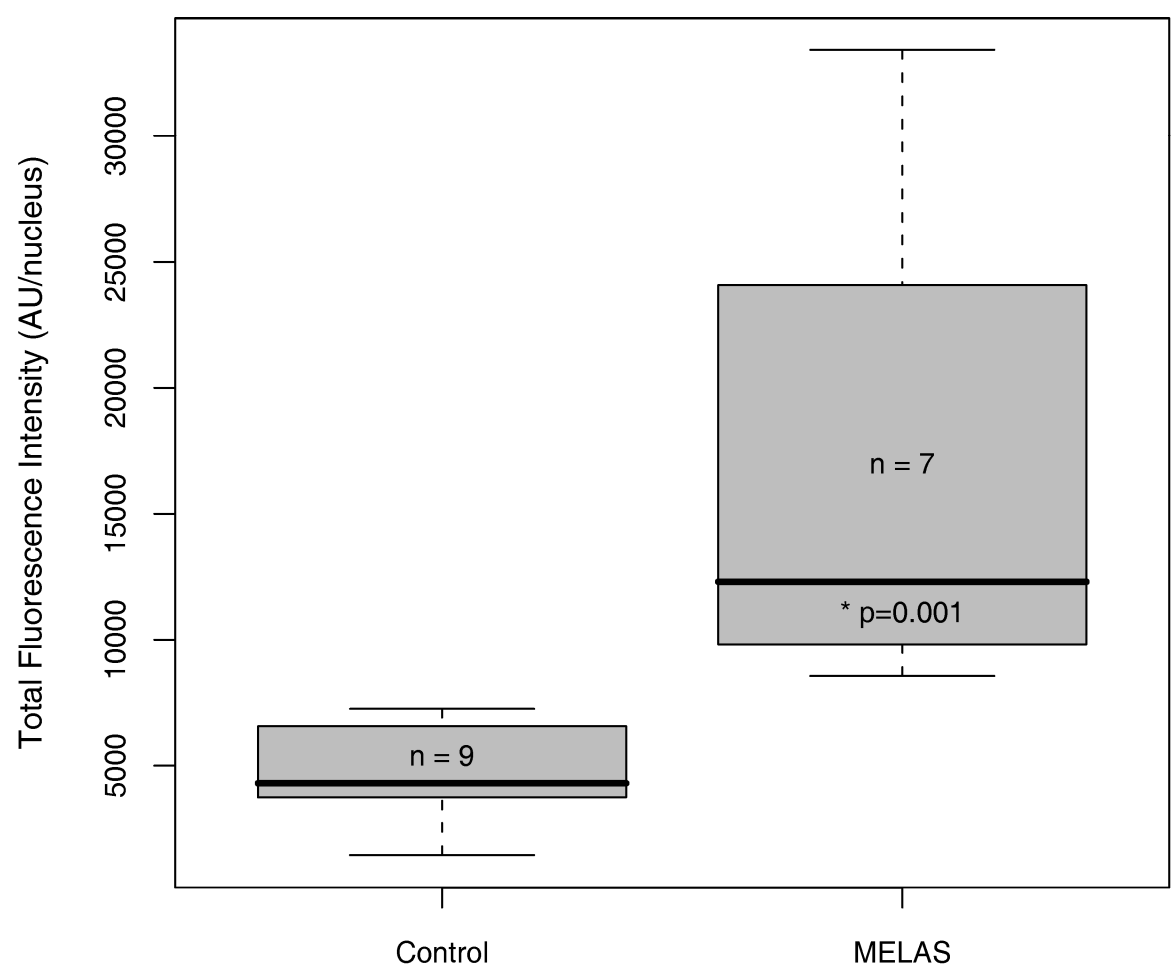

Group

\section{Figure 1: DHE staining}

Cryosections of skeletal muscle samples of controls $(n=9)$ and MELAS m.3243A $>C$ mutation carriers $(n=7)$ were stained with dihydroethidium (DHE) as described in the Materials and Methods section to assess the activity of enzymes able to produce superoxide radicals. A one-sided t-test showed that the expression of these enzymes in muscle of $\mathrm{m} .3243 \mathrm{~A}>\mathrm{G}$ mutation carriers was significantly 3.8-fold higher than in controls $(p=0.001)$. The whiskers in the box plot indicate the extreme values. The boxes indicate the median (thick line) and the interquartile ranges (grey area).

AU: arbitrary units, reflecting the total fluorescence intensity of all events divided by the number of blue fluorescent nuclei (see Materials and Methods section). 


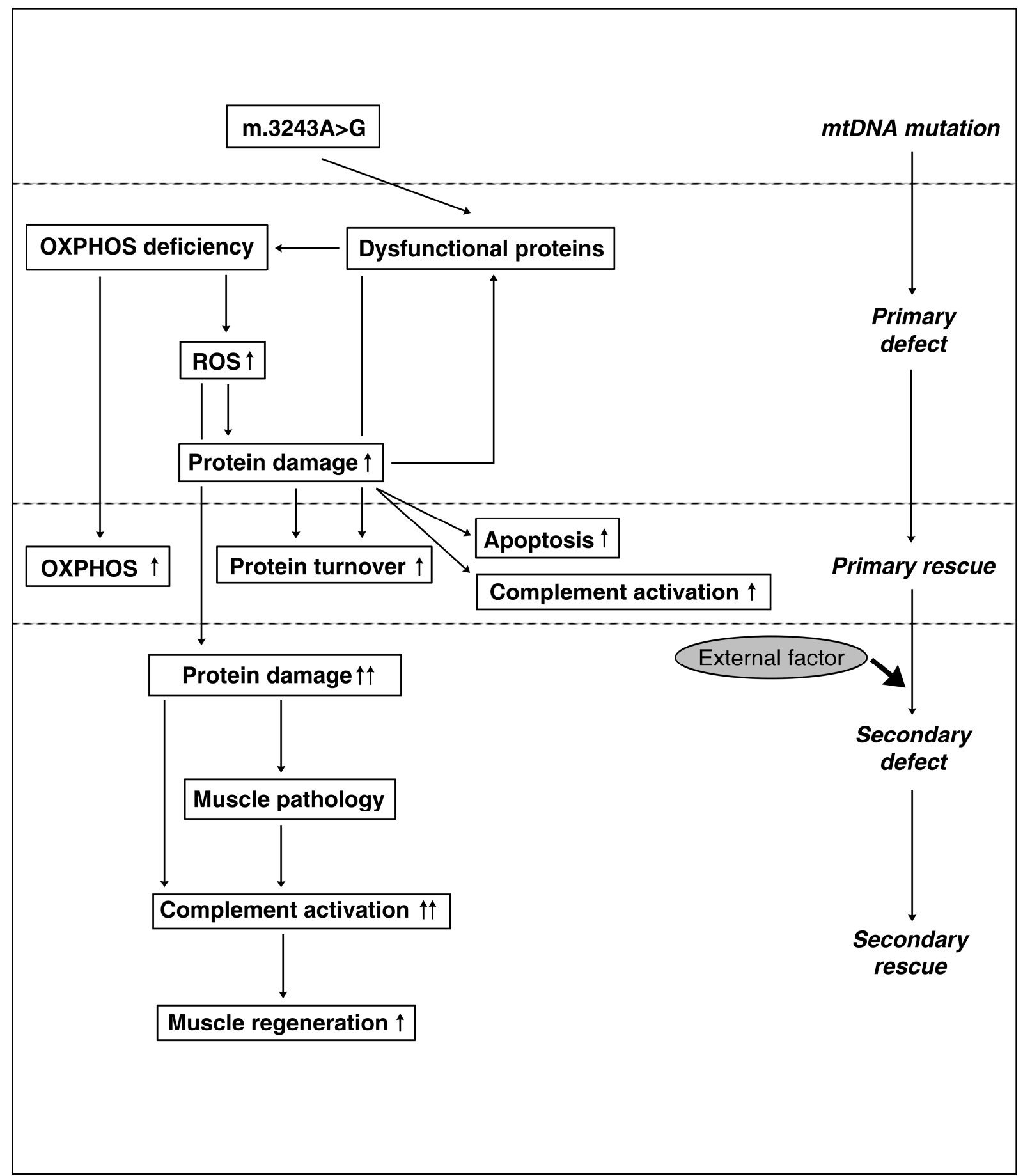

Figure 2: Pathobiological process flow of the m.3243A>G mutation

The m.3243A>G mutation gives rise to dysfunctional and damaged proteins. As a primary rescue attempt to this primary defect, OXPHOS is stimulated, protein turnover and apoptosis are increased, and the complement system is activated to initiate muscle regeneration. When the primary rescue mechanism fails, probably due to irreparable damage, it is terminated, protein damage increases further and muscle pathology arises as a secondary stage. The severe muscle pathology and increasing protein damage triggers a secondary rescue attempt through strengthening of complement activation, which may stimulate muscle regeneration further as a sole rescue process. 


\section{Discussion}

Gene expression studies have been performed before on muscle of patients carrying the m.3243A>G mutation, in comparison to controls and other mtDNA mutations [118, 119]. In contrast to our study, no a-symptomatic mutation carriers were included. In the first study only specific processes were evaluated in muscle of three symptomatic MELAS patients carrying the m. $3243 A>C$ mutation (mutation loads of $50 \%, 72 \%$, and $91 \%$ ) compared to controls. Only in the patient with the highest mutation load, an induction of OXPHOS genes, genes involved in energy metabolism (glycolysis, glycogen metabolism, citric acid cycle), and ubiquitin mRNA levels indicating an increased protein turnover, was reported [118]. No gene expression changes in these processes were identified for the other two subjects. In contrast to our findings, these authors observe an increase in gene expression with mutation load. In the second study, which also investigated symptomatic MELAS patients carrying the m.3243A>C mutation compared to controls, genes related to arginine metabolism were up-regulated and genes involved in cell cycle regulation and apoptosis were up- and down-regulated indicating both a stimulation and an inhibition of these processes. No reference was made to an induction of OXPHOS genes in patients compared to controls [119]. In agreement with the results of the studies described above, our results indicate a minor stimulation of the OXPHOS system, but in a-symptomatic m.3243A>C mutation carriers only. Additionally, our results showed mixed changes in the apoptotic process and an increase in protein turnover. However, no significant gene expression changes were identified in glycogen metabolism, glycolysis, or the citric acid cycle. We were not able to identify gene expression changes concerning arginine metabolism. The arginine metabolism genes which were represented in our dataset were not differentially expressed between m.3243A $>\mathrm{G}$ mutation carriers and controls, but not all genes involved were covered in our dataset.

Our data has revealed a number of processes that occur in carriers of the m.3243A $>G$ mutation. The most striking observations were the difference between the a-symptomatic and symptomatic carriers and which has not been reported before, the involvement of the complement system. The pathophysiological processes which can be derived from our data are depicted in figure 2 and the individual components of this process will be discussed below.

Compensation by increasing OXPHOS capacity in a-symptomatic m.3243A $>\mathrm{G}$ mutation carriers

A slight up-regulation of structural subunits of OXPHOS complexes I and IV, which are the most commonly deficient respiratory chain complexes in MELAS patients, is observed in a-symptomatic carriers of the m.3243A $>G$ mutation, probably to compensate for the OXPHOS defect. This is in contrast to the $\mathrm{S} 3243$ subjects, where no gene expression changes for subunits of complex I and IV and even a down-regulation of the gene coding for the $\mathrm{ATP}_{5} \mathrm{C}_{1}$ subunit of the mitochondrial ATP synthase complex was detected. This is similar to patients having Leigh syndrome caused by 
a mutation in the SURF1 gene, except for the down-regulation of the SURF1 gene itself (unpublished results). Additionally, compensation by stimulation of mitochondrial proliferation in the a-symptomatic m.3243A>C mutation carriers compared to controls could be identified, illustrated by the small but significant 1.1-fold up-regulation of the PPRC1 gene (Peroxisome proliferative activated receptor, gamma, coactivator-related 1), as has been suggested before [120]. PPRC1 is similar to PPAR-gamma coactivator 1 (PPARGC1/PGC-1) which plays a role in the activation of mitochondrial biogenesis [121, 122], but in contrast to PGC-1 it is growth-regulated with the characteristic of an immediate early gene product. Given these results, it is likely that the up-regulation of OXPHOS structural subunits and mitochondrial biogenesis is a primary rescue attempt.

\section{Stimulation of protein turnover in $m .3243 A>G$ mutation carriers}

Stimulation of protein synthesis is a general process triggered by the $\mathrm{m} .3243 \mathrm{~A}>\mathrm{G}$ mutation, irrespective of the presence of clinical symptoms, possibly related to alterations in the translational process. A reduced leucine amino acid incorporation has been described in MELAS m.3243A $>C$ subjects which results in dysfunctional protein production and protein synthesis rate reduction [103, 123]. The second DAVID cluster for the Mut3243 group (table 4) indicates upregulation of protein synthesis, by up-regulation of ribosome and ER related genes (RPL23, TRAM2, and RRBP1). The up-regulation of the TRAM2 gene might also be related to the disturbed calcium homeostasis in MELAS subjects, since the TRAM2 protein appears to interact with the $\mathrm{Ca}(2+)$ pump of the ER (SERCA2b) $[107,124]$. Up-regulation of protein synthesis by a significant upregulation of three translation initiation factors (EIF2AK1, EIF2B4, and EIF2S1), a subunit of the ribosomal $40 S$ complex (RPS21), and a mitochondrial ribosomal protein (MRPL23) is a very prominent process in a-symptomatic carriers, probably in an effort to rebalance the dysfunctional protein content (see below). Investigation of the protein synthesis machinery in more detail shows that the majority of the other transcripts present in the dataset coding for members of the ribosomal subunits $40 S$ and $60 S$ had a tendency to be up-regulated as well. Eight out of fifteen transcripts for which data was available of the 60S ribosomal subunit (RPL10A, RPL13, RPL14, RPL18, RPL23, RPL24, RPL27, and RPL32) and four out of thirteen transcripts for which data was available of the 40 S ribosomal subunit (RPS6, RPS11, RPS23, and RPS24) showed a trend to be more than 1.2-fold up-regulated. Although these changes were not significant on a single gene level, it suggests that the protein synthesis process as a whole is stimulated.

Bases on our data we hypothesise (figure 2) that dysfunctional proteins are degraded and replaced by new proteins, resulting in a stimulation of protein synthesis and intracellular protein trafficking. In line with this hypothesis, five out of nine transcripts of the $20 \mathrm{~S}$ proteasome complex (PSMA4, PSMA5, PSMB1, PSMB4, and PSMB5), which is mainly associated with the breakdown of proteins with oxidative damage [83], showed a trend to be more than 1.2-fold upregulated in a-symptomatic m.3243A $>\mathrm{G}$ mutation carriers compared to controls. Moreover, PSME1 
and PSME2, both regulators of the 205 proteasome complex, showed a tendency to be more than 1.2-fold up-regulated. Stimulation of protein breakdown in the AS3243 group is further supported by the significant up-regulation of two transcripts from the fourth functional gene cluster from the DAVID analysis of which the function is related to lysosomal degradation of proteins. CHMP2A, which is involved in the formation of multivesicular bodies delivering transmembrane proteins into the lumen of the lysosome for degradation, was significantly up-regulated $[125,126]$. GGA3 was also significantly up-regulated and belongs to a protein family that regulates the trafficking of membrane proteins between the trans-Golgi network and the lysosome by binding to ubiquitin attached to membrane proteins [127]. Stimulation of protein synthesis and degradation was not detected in the $\$ 3243$ group compared to controls, as no up-regulation of ribosomal subunits and no stimulation of the proteasomal protein degradation pathway was observed for this patient group. Even a subtle down-regulation (1.1-fold compared to controls) of two translation initiation factors (EIF2B5 and EIF4G1) was observed in the S3243 group compared to controls.

A strong additional trigger for the stimulation of protein synthesis is increased oxidative protein damage, due to the impaired OXPHOS function. A significant higher amount of oxidatively damaged proteins and increased expression of ROS-producing enzymes was detected in muscle of m.3243A $>G$ mutation carriers compared to controls (figure 1). The fact that the ability to produce ROS was increased at similar levels in symptomatic and a-symptomatic mutation carriers compared to controls, corresponds with the results of the oxprot assay, which indicated equal oxidative protein damage in symptomatic and a-symptomatic mutation carriers. Because the amount of carbonyl groups per mg protein for one a-symptomatic subject was much less than for the other two a-symptomatic subjects, one might suggest that some a-symptomatic mutation carriers might be able to more efficiently replace damaged proteins with functional ones compared to the symptomatic mutation carriers.

\section{Apoptosis in m.3243A>G mutation carriers}

Gene expression changes indicate both an inhibition and a stimulation of programmed cell death as a general effect of the m.3243A $>C$ mutation (table 4), which might reflect heterogeneity among the carriers. For the a-symptomatic m.3243A $>\mathrm{G}$ mutation carriers compared to controls, there appears to be a pro-apoptotic effect of the gene expression changes (table 5). No changes in the apoptosis process have been identified for the $\mathrm{S} 3243$ group separately. Apoptosis has previously been reported in MELAS subjects with the m.3243A>C mutation and in OXPHOS disorders in general and was linked to cell proliferation and high mutation loads [120]. The trigger for apoptosis is not completely clear, but excessive ROS production leading to increased protein and cell damage beyond repair and malfunctioning due to a defective oxidative phosphorylation system resulting in energy deprivation might be involved. Possibly, during disease progression, the slight stimulation of apoptosis stops. An explanation might be that when the protein damage is less severe, damaged proteins can still be removed by increasing protein turnover followed by 
the stimulation of apoptosis and muscle regeneration through complement activation (see below). When protein damage can not be repaired or removed, it persists probably to save energy for regeneration, and muscle pathology then manifests (figure 2).

Muscle regeneration by complement activation in $m .3243 A>G$ mutation carriers

In the m.3243A>G mutation carriers, a number of complement related genes were up-regulated. Microarray analysis showed that complement component $1 \mathrm{r}\left(\mathrm{C}_{1} R\right)$, and clusterin (CLU) were significantly up-regulated. QRT-PCR confirmed these findings and also indicated a significant upregulation of complement component $1 \mathrm{~s}$ (C1S) and complement component 3 (C3). These changes were specific for mutation carriers in general, irrespective of having symptoms or not. The up-regulation of complement components most likely reflects a process of muscle regeneration comparable to that observed in Leigh syndrome patients with a SURF1 mutation (unpublished data). The 1.2 fold up-regulation of the RPB1 gene, which is specifically expressed in neuromuscular junctions and showed a transient induction in concordance with previously known neuromuscular junction markers in a 27-time point muscle regeneration series experiment [128], is also related to regenerative processes [129]. The significantly stronger up-regulation of $C_{1} R$ for the symptomatic compared to the a-symptomatic mutation carriers (6.8-fold compared to 2.7-fold) may be due to the increasing damage to proteins, which is not removed anymore. This is bound to fail, as muscle damage prevails and the new muscle carries the same genetic defect. However, restoration of the wild type mtDNA has been reported in regenerating muscle fibres, some of which are mtDNA mutation free [130].

\section{Conclusion}

It appears that the detrimental effects of the m.3243A>C mutation can be compensated by upregulation of genes coding for structural subunits of OXPHOS complexes and by stimulation of mitochondrial biogenesis, protein turnover, apoptosis, and muscle regeneration. When these compensating mechanisms fall short and are terminated, pathology becomes manifest and eventually regeneration of failing muscle by complement activation is triggered even further (figure 2). It is yet unclear which factors determine successful compensation. Mutation load may play a role, but as the mutation load of two of the investigated a-symptomatic m.3243A>C mutation carriers (subjects 25 and 30, table 1) fall in the higher mutation load range (60\% and $65 \%$ respectively) of the symptomatic mutation carriers (32\% - 73\%) and one of the symptomatic subjects (subject 17, table 1) had a mutation load of $32 \%$, falling in the lower range of the a-symptomatic subjects, this can not be the only factor involved. The involvement of (yet unknown) genetic and environmental factors has been proposed [99]. It is striking that a-symptomatic subject 25 (60\% mutation load, table 1$)$ is a fanatic sportsman who runs marathons. Physical exercise is known to stimulate mitochondrial proliferation in patients with mitochondrial myopathy [131], and therefore might have a favourable effect on this compensatory mechanism, as long as no symptoms are present. Moreover, it is well known that physical exercise delays the 
reduction of muscle mass during aging and therefore might support the regenerative process which is stimulated in m.3243A>C mutation carriers by complement activation. Physical exercise has also been suggested as a tool to stimulate muscle regeneration in order to increase the proportion of wild-type mtDNA molecules in the muscle tissue. Exercise would lead to specific necrosis of exercise muscle fibres, which in turn would trigger muscle fibre regeneration populating the new muscle fibres with predominantly wild-type mtDNA molecules [132]. Therapeutic interventions aimed at stimulating mitochondrial proliferation, muscle regeneration and reducing ROS damage will be beneficial to delay the onset of symptoms in m.3243A>C carriers. These interventions should start at early age, before symptoms become manifest, as their effect may be detrimental for already affected patients. As protein turnover, apoptosis, and complement activation, with in specific the expression of the $C_{1} R$ gene, differ between the symptomatic and a-symptomatic m.3243A $>\mathrm{G}$ mutation carriers, the expression of these genes may be markers for the severity and progression of MELAS disease.

\section{Acknowledgements}

This research was supported by the Princess Beatrix Foundation, (Grant number: MAR99-0111) and the MitoCircle project (EU grant, Sixth Framework Program, contr. no. 005260). We would like to thank Mr. Pieter Derkx of the Pathology department at Erasmus MC, Rotterdam, The Netherlands, for the preparation of the muscle cryosections. 
Supplementary table S1: Significantly differentially expressed genes

\begin{tabular}{|c|c|c|c|}
\hline $\begin{array}{l}\text { Unigene } \\
\text { ID }\end{array}$ & $\begin{array}{l}\text { Fold- } \\
\text { change }\end{array}$ & Gene Description & $\begin{array}{l}\text { Gene } \\
\text { Symbol }\end{array}$ \\
\hline \multicolumn{4}{|l|}{ S-3243 } \\
\hline Hs.498178 & 0.6 & Actinin, alpha 2 & ACTN2 \\
\hline Hs.82609 & 0.7 & Hydroxymethylbilane synthase & HMBS \\
\hline Hs.367992 & 0.7 & Inositol(myo)-1(or 4)-monophosphatase 2 & IMPA2 \\
\hline Hs.101337 & 0.7 & Uncoupling protein 3 (mitochondrial, proton carrier) & UCP3 \\
\hline Hs.440960 & 0.7 & RAD23 homolog A (S. cerevisiae) & RAD23A \\
\hline Hs.12084 & 0.7 & Tu translation elongation factor, mitochondrial & TUFM \\
\hline Hs.406678 & 0.8 & Acyl-CoA synthetase long-chain family member 1 & ACSL1 \\
\hline Hs. 13640 & 0.8 & Roundabout, axon guidance receptor, homolog 1 (Drosophila) & ROBO1 \\
\hline Hs. 515274 & 0.8 & Splicing factor 4 & SF4 \\
\hline Hs. 6651 & 0.8 & Vesicle-associated membrane protein 4 & VAMP4 \\
\hline Hs.132902 & 0.8 & CAP, adenylate cyclase-associated protein, 2 (yeast) & CAP2 \\
\hline Hs. 514303 & 0.8 & Prohibitin & PHB \\
\hline Hs. 642970 & 0.8 & Transcribed locus & \\
\hline Hs.43666 & 0.8 & Protein tyrosine phosphatase type IVA, member 3 & PTP4A3 \\
\hline Hs.465985 & 0.8 & ArsA arsenite transporter, ATP-binding, homolog 1 (bacterial) & ASNA1 \\
\hline Hs. 271135 & 0.8 & ATP synthase, $\mathrm{H}+$ transporting, mitochondrial F1 complex, gamma polypeptide 1 & ATP5C1 \\
\hline Hs.36587 & 0.8 & Protein phosphatase 1 , regulatory subunit 7 & PPP1R7 \\
\hline Hs.283551 & 0.8 & Eukaryotic translation initiation factor $2 \mathrm{~B}$, subunit 5 epsilon, $82 \mathrm{kDa}$ & EIF2B5 \\
\hline Hs.433750 & 0.8 & Eukaryotic translation initiation factor 4 gamma, 1 & EIF4G1 \\
\hline Hs.52788 & 0.8 & Fragile $\mathrm{X}$ mental retardation, autosomal homolog 2 & FXR2 \\
\hline Hs. 534338 & 0.8 & Protein phosphatase 4 (formerly X), catalytic subunit & PPP4C \\
\hline Hs. 513522 & 0.8 & Fusion (involved in $\mathrm{t}(12 ; 16)$ in malignant liposarcoma) & FUS \\
\hline Hs. 515139 & 0.8 & Transmembrane emp24 protein transport domain containing 1 & TMED1 \\
\hline Hs. 54460 & 0.9 & Chemokine (C-C motif) ligand 11 & CCL11 \\
\hline Hs. 632340 & 0.9 & $\begin{array}{l}\text { Methylenetetrahydrofolate dehydrogenase (NADP+ dependent) } 1, \text { methenyltetrahydrofolate } \\
\text { cyclohydrolase, } \\
\text { formyltetrahydrofolate synthetase }\end{array}$ & MTHFD1 \\
\hline Hs.643147 & 0.9 & Transcribed locus & \\
\hline Hs.37092 & 0.9 & Fibroblast growth factor 3 (murine mammary tumor virus integration site (v-int-2) oncogene homolog) & FGF3 \\
\hline Hs.419815 & 0.9 & Epidermal growth factor (beta-urogastrone) & EGF \\
\hline Hs.69089 & 0.9 & Galactosidase, alpha & GLA \\
\hline Hs. 595187 & 0.9 & Transcribed locus & \\
\hline Hs. 597347 & 0.9 & Transcribed locus & \\
\hline Hs. 584887 & 0.9 & Gamma tubulin ring complex protein (76p gene) & $76 \mathrm{P}$ \\
\hline Hs. 593420 & 0.9 & MRNA; cDNA DKFZp564G112 (from clone DKFZp564G112) & \\
\hline Hs. 631863 & 0.9 & Neuropathy target esterase & PNPLA6 \\
\hline Hs.246381 & 0.9 & $\begin{array}{l}\text { Transcribed locus, strongly similar to NP_001242.1 CD68 antigen; Macrophage antigen CD68 } \\
\text { (microsialin); } \\
\text { macrosialin; scavenger receptor class D, member } 1 \text { [Homo sapiens] }\end{array}$ & \\
\hline Hs.533022 & 0.9 & Crystallin, beta B3 & CRYBB3 \\
\hline Hs.332422 & 0.9 & Aspartate beta-hydroxylase & ASPH \\
\hline Hs. 94367 & 0.9 & Thyroid transcription factor 1 & TITF1 \\
\hline Hs. 532188 & 0.9 & Integrator complex subunit 1 & INTS1 \\
\hline Hs. 94395 & 0.9 & ATP-binding cassette, sub-family D (ALD), member 4 & $\mathrm{ABCD} 4$ \\
\hline Hs.445489 & 0.9 & Pleckstrin homology domain containing, family B (evectins) member 1 & PLEKHB1 \\
\hline Hs.464469 & 0.9 & Myomesin 1 (skelemin) $185 \mathrm{kDa}$ & MYOM1 \\
\hline Hs.37023 & 0.9 & Growth hormone releasing hormone & GHRH \\
\hline Hs.584806 & 0.9 & Transcription elongation factor B (SIII), polypeptide 3 (110kDa, elongin A) & TCEB3 \\
\hline Hs.50727 & 0.9 & Hydroxysteroid (17-beta) dehydrogenase 1 & HSD17B1 \\
\hline Hs. 408458 & 0.9 & WW domain containing E3 ubiquitin protein ligase 2 & WWP2 \\
\hline Hs. 133444 & 0.9 & PiggyBac transposable element derived 3 & PGBD3 \\
\hline Hs. 471405 & 0.9 & Tubulin tyrosine ligase-like family, member 4 & TTLL4 \\
\hline Hs. 432818 & 0.9 & UDP-N-acetyl-alpha-D-galactosamine:polypeptide N-acetylgalactosaminyltransferase 10 (GalNAc-T10) & GALNT10 \\
\hline Hs.591379 & 0.9 & DOT1-like, histone H3 methyltransferase (S. cerevisiae) & DOT1L \\
\hline
\end{tabular}




\begin{tabular}{|c|c|c|c|}
\hline Hs.122908 & 0.9 & Chromatin licensing and DNA replication factor 1 & CDT1 \\
\hline Hs.198249 & 0.9 & Gap junction protein, beta 5 (connexin 31.1) & GJB5 \\
\hline Hs.74615 & 1.1 & Platelet-derived growth factor receptor, alpha polypeptide & PDGFRA \\
\hline Hs.24587 & 1.1 & Embryonal Fyn-associated substrate & EFS \\
\hline Hs.159223 & 1.1 & NGFI-A binding protein 2 (EGR1 binding protein 2) & NAB2 \\
\hline Hs.618002 & 1.2 & Transcribed locus & \\
\hline Hs.128548 & 1.2 & WD repeat domain 1 & WDR1 \\
\hline Hs.390567 & 1.2 & FYN oncogene related to SRC, FGR, YES & FYN \\
\hline Hs.98594 & 1.2 & Rho guanine nucleotide exchange factor (GEF) 10 & ARHGEF10 \\
\hline Hs.202097 & 1.3 & Procollagen C-endopeptidase enhancer & PCOLCE \\
\hline Hs.174195 & 1.3 & Interferon induced transmembrane protein 2 (1-8D) & IFITM2 \\
\hline Hs.508716 & 1.4 & Collagen, type IV, alpha 2 & COL4A2 \\
\hline Hs.296049 & 1.4 & Microfibrillar-associated protein 4 & MFAP4 \\
\hline Hs.513022 & 1.5 & Immunoglobulin superfamily containing leucine-rich repeat & ISLR \\
\hline Hs.458573 & 1.6 & Platelet-derived growth factor receptor-like & PDGFRL \\
\hline Hs.593775 & 1.8 & NA & NA \\
\hline Hs.103253 & 2.2 & Perilipin & PLIN \\
\hline \multicolumn{4}{|l|}{ AS-3243 } \\
\hline Hs.116244 & 0.7 & WD repeat domain 62 & WDR62 \\
\hline Hs.124940 & 0.8 & Rho family GTPase 1 & RND1 \\
\hline Hs.642952 & 0.8 & SRY (sex determining region Y)-box 12 & SOX12 \\
\hline Hs.2200 & 0.8 & Perforin 1 (pore forming protein) & PRF1 \\
\hline Hs.442658 & 0.8 & Aurora kinase $\mathrm{B}$ & AURKB \\
\hline Hs.369759 & 0.8 & Transient receptor potential cation channel, subfamily M, member 2 & TRPM2 \\
\hline Hs.513484 & 0.9 & Quinolinate phosphoribosyltransferase (nicotinate-nucleotide pyrophosphorylase (carboxylating)) & QPRT \\
\hline Hs.416848 & 0.9 & Cathepsin W (lymphopain) & CTSW \\
\hline Hs.159309 & 0.9 & Uroplakin $1 \mathrm{~A}$ & UPK1A \\
\hline Hs.27183 & 0.9 & Chromosome 14 open reading frame 79 & C14orf79 \\
\hline Hs. 87247 & 0.9 & Harakiri, BCL2 interacting protein (contains only BH3 domain) & HRK \\
\hline Hs. 531563 & 0.9 & Transmembrane protein 15 & TMEM15 \\
\hline Hs.370267 & 1.1 & Tankyrase, TRF1-interacting ankyrin-related ADP-ribose polymerase & TNKS \\
\hline Hs.100299 & 1.1 & Ligase III, DNA, ATP-dependent & LIG3 \\
\hline Hs.250712 & 1.1 & Calcium channel, voltage-dependent, beta 3 subunit & CACNB3 \\
\hline Hs. 513470 & 1.1 & Nuclear factor of activated T-cells, cytoplasmic, calcineurin-dependent 2 interacting protein & NFATC2IP \\
\hline Hs.461361 & 1.1 & Craniofacial development protein 1 & CFDP1 \\
\hline Hs.153022 & 1.1 & TATA box binding protein (TBP)-associated factor, RNA polymerase I, C, 110kDa & TAF1C \\
\hline Hs.1314 & 1.1 & Tumor necrosis factor receptor superfamily, member 8 & TNFRSF8 \\
\hline Hs.487294 & 1.1 & $\begin{array}{l}\text { Excision repair cross-complementing rodent repair deficiency, complementation group } 2 \\
\text { (xeroderma pigmentosum D) }\end{array}$ & ERCC2 \\
\hline Hs.119689 & 1.1 & Glycoprotein hormones, alpha polypeptide & CGA \\
\hline Hs.631503 & 1.1 & Ectonucleoside triphosphate diphosphohydrolase 6 (putative function) & ENTPD6 \\
\hline Hs.528299 & 1.1 & HIV-1 Tat interacting protein, $60 \mathrm{kDa}$ & HTATIP \\
\hline Hs.533551 & 1.1 & Peroxisome proliferative activated receptor, gamma, coactivator-related 1 & PPRC1 \\
\hline Hs. 546250 & 1.1 & Dynein, cytoplasmic 1 , intermediate chain 2 & DYNC112 \\
\hline Hs.268887 & 1.1 & Serine/threonine kinase 17a (apoptosis-inducing) & STK17A \\
\hline Hs.19012 & 1.1 & Rab9 effector protein with kelch motifs & RABEPK \\
\hline Hs.515598 & 1.1 & PRP31 pre-mRNA processing factor 31 homolog (S. cerevisiae) & PRPF31 \\
\hline Hs.183800 & 1.1 & Ran GTPase activating protein 1 & RANGAP1 \\
\hline Hs.591054 & 1.1 & $\mathrm{BH} 3$ interacting domain death agonist & BID \\
\hline Hs. 557646 & 1.1 & Cyclin-dependent kinase 9 (CDC2-related kinase) & CDK9 \\
\hline Hs. 602240 & 1.1 & Transcribed locus & \\
\hline Hs.463105 & 1.1 & DEAH (Asp-Glu-Ala-His) box polypeptide 8 & DHX8 \\
\hline Hs.408312 & 1.1 & Tumor protein p53 (Li-Fraumeni syndrome) & TP53 \\
\hline Hs. 642621 & 1.1 & NA & NA \\
\hline Hs.591189 & 1.1 & KIAA0100 & KIAA0100 \\
\hline Hs.2549 & 1.1 & Adrenergic, beta-3-, receptor & ADRB3 \\
\hline
\end{tabular}




\begin{tabular}{|c|c|c|c|}
\hline Hs.441975 & 1.1 & XIAP associated factor-1 & BIRC4BP \\
\hline Hs. 513913 & 1.1 & GABA(A) receptor-associated protein & GABARAP \\
\hline Hs.493309 & 1.1 & KIAA0020 & KIAA0020 \\
\hline Hs.386470 & 1.1 & Neuromedin B & NMB \\
\hline Hs. 606016 & 1.1 & Twist homolog 1 (acrocephalosyndactyly 3; Saethre-Chotzen syndrome) (Drosophila) & TWIST1 \\
\hline Hs.592984 & 1.1 & Scavenger receptor class $B$, member 2 & SCARB2 \\
\hline Hs. 159556 & 1.1 & MTERF domain containing 2 & MTERFD2 \\
\hline Hs.632368 & 1.1 & Exosome component 10 & EXOSC10 \\
\hline Hs. 525629 & 1.1 & Metastasis associated 1 & MTA1 \\
\hline Hs. 444356 & 1.1 & Growth factor receptor-bound protein 2 & GRB2 \\
\hline Hs.81134 & 1.1 & Interleukin 1 receptor antagonist & IL1RN \\
\hline Hs. 466044 & 1.1 & Protein kinase N1 & PKN1 \\
\hline Hs. 517517 & 1.1 & E1A binding protein $\mathrm{p} 300$ & EP300 \\
\hline Hs. 518138 & 1.1 & KIAA0040 & KIAA0040 \\
\hline Hs. 591946 & 1.1 & Matrix metallopeptidase 20 (enamelysin) & MMP20 \\
\hline Hs.437599 & 1.1 & Hermansky-Pudlak syndrome 5 & HPS5 \\
\hline Hs.133539 & 1.1 & Microtubule associated serine/threonine kinase family member 4 & MAST4 \\
\hline Hs.35490 & 1.1 & KIAA0350 protein & KIAA0350 \\
\hline Hs.567261 & 1.2 & MAP/microtubule affinity-regulating kinase 2 & MARK2 \\
\hline Hs.198853 & 1.2 & Chromosome 12 open reading frame 32 & C12orf32 \\
\hline Hs.126714 & 1.2 & Class II, major histocompatibility complex, transactivator & CIITA \\
\hline Hs.195667 & 1.2 & KIAA0329 & KIAA0329 \\
\hline Hs.473296 & 1.2 & Tumor protein D52-like 2 & TPD52L2 \\
\hline Hs. 607883 & 1.2 & Transcribed locus & \\
\hline Hs. 132370 & 1.2 & Cleavage stimulation factor, $3^{\prime}$ pre-RNA, subunit $2,64 \mathrm{kDa}$ & CSTF2 \\
\hline Hs.493202 & 1.2 & Cleavage and polyadenylation specific factor $1,160 \mathrm{kDa}$ & CPSF1 \\
\hline Hs.352614 & 1.2 & 1-acylglycerol-3-phosphate 0-acyltransferase 7 (lysophosphatidic acid acyltransferase, eta) & AGPAT7 \\
\hline Hs. 172865 & 1.2 & Cleavage stimulation factor, 3 ' pre-RNA, subunit $1,50 \mathrm{kDa}$ & CSTF1 \\
\hline Hs.592088 & 1.2 & Zinc finger protein 205 & ZNF205 \\
\hline Hs.5920 & 1.2 & Glucosamine (UDP-N-acetyl)-2-epimerase/N-acetylmannosamine kinase & GNE \\
\hline Hs.632905 & 1.2 & Transcribed locus & \\
\hline Hs. 472877 & 1.2 & Eyes absent homolog 2 (Drosophila) & EYA2 \\
\hline Hs.272822 & 1.2 & RuvB-like 1 (E. coli) & RUVBL1 \\
\hline Hs.10095 & 1.2 & Myeloid/lymphoid or mixed-lineage leukemia (trithorax homolog, Drosophila); translocated to, 1 & MLLT1 \\
\hline Hs. 530940 & 1.2 & $\begin{array}{l}\text { Transcribed locus, strongly similar to XP_543397.2 PREDICTED: similar to BRCA1 associated protein } \\
\text { [Canis familiaris] }\end{array}$ & \\
\hline Hs. 279913 & 1.2 & Processing of precursor 5 , ribonuclease P/MRP subunit (S. cerevisiae) & POP5 \\
\hline Hs.631863 & 1.2 & Neuropathy target esterase & PNPLA6 \\
\hline Hs.592048 & 1.2 & Ubiquinol-cytochrome c reductase core protein II & UQCRC2 \\
\hline Hs.297304 & 1.2 & Glycosyltransferase 8 domain containing 1 & GLT8D1 \\
\hline Hs.505033 & 1.2 & V-Ki-ras2 Kirsten rat sarcoma viral oncogene homolog & KRAS \\
\hline Hs.397918 & 1.2 & $\mathrm{KIN}$, antigenic determinant of recA protein homolog (mouse) & KIN \\
\hline Hs.520205 & 1.2 & Eukaryotic translation initiation factor 2-alpha kinase 1 & EIF2AK1 \\
\hline Hs.84113 & 1.2 & Cyclin-dependent kinase inhibitor 3 (CDK2-associated dual specificity phosphatase) & CDKN3 \\
\hline Hs.485471 & 1.2 & CDC5 cell division cycle 5-like (S. pombe) & CDC5L \\
\hline Hs. 631240 & 1.2 & Transcribed locus & \\
\hline Hs.591936 & 1.2 & Signal recognition particle receptor ('docking protein') & SRPR \\
\hline Hs.9383 & 1.2 & Cysteine-rich with EGF-like domains 1 & CRELD1 \\
\hline Hs.300404 & 1.2 & Esophageal cancer associated protein & MGC16824 \\
\hline Hs. 554831 & 1.2 & Polymerase (RNA) II (DNA directed) polypeptide D & POLR2D \\
\hline Hs. 500340 & 1.2 & Thyroid hormone receptor interactor 4 & TRIP4 \\
\hline Hs.159525 & 1.2 & Cell growth regulator with EF-hand domain 1 & CGREF1 \\
\hline Hs. 558473 & 1.2 & Chromosome 18 open reading frame 10 & C18orf10 \\
\hline Hs. 575381 & 1.2 & Ankyrin repeat domain 6 & ANKRD6 \\
\hline Hs. 270043 & 1.2 & KIAA0196 & KIAA0196 \\
\hline
\end{tabular}




\begin{tabular}{|c|c|c|c|}
\hline Hs.631814 & 1.2 & La ribonucleoprotein domain family, member 5 & LARP5 \\
\hline Hs.84549 & 1.2 & NADH dehydrogenase (ubiquinone) 1, subcomplex unknown, 1, 6kDa & NDUFC1 \\
\hline Hs.606668 & 1.2 & Transcribed locus & \\
\hline Hs.264482 & 1.2 & ATG12 autophagy related 12 homolog (S. cerevisiae) & ATG12 \\
\hline Hs.368971 & 1.2 & Nuclear receptor coactivator 6 & NCOA6 \\
\hline Hs.1030 & 1.2 & Ras and Rab interactor 1 & RIN1 \\
\hline Hs.595201 & 1.2 & Transcribed locus & \\
\hline Hs.204041 & 1.2 & AHA1, activator of heat shock 90kDa protein ATPase homolog 1 (yeast) & AHSA1 \\
\hline Hs.102308 & 1.2 & Potassium inwardly-rectifying channel, subfamily J, member 8 & KCNJ8 \\
\hline Hs.154073 & 1.2 & Solute carrier family 35 , member B1 & SLC35B1 \\
\hline Hs.494192 & 1.2 & Osteoclast stimulating factor 1 & OSTF1 \\
\hline Hs.808 & 1.2 & Heterogeneous nuclear ribonucleoprotein $\mathrm{F}$ & HNRPF \\
\hline Hs.397465 & 1.2 & Homeodomain interacting protein kinase 2 & HIPK2 \\
\hline Hs. 10848 & 1.2 & BMS1-like, ribosome assembly protein (yeast) & BMS1L \\
\hline Hs.580681 & 1.2 & SAM domain and HD domain 1 & SAMHD1 \\
\hline Hs.436646 & 1.2 & Sodium channel, voltage-gated, type I, beta & SCN1B \\
\hline Hs.532492 & 1.2 & Acid phosphatase 2, lysosomal & ACP2 \\
\hline Hs.412870 & 1.2 & $\begin{array}{l}\text { Transcribed locus, strongly similar to NP_006618.1 POP4 (processing of precursor, S. cerevisiae) } \\
\text { homolog; } 1110023 \text { P21Rik [Homo sapiens] }\end{array}$ & \\
\hline Hs.530251 & 1.2 & TAF12 RNA polymerase II, TATA box binding protein (TBP)-associated factor, 20kDa & TAF12 \\
\hline Hs.180711 & 1.2 & Syntaxin 3 & STX3 \\
\hline Hs.485081 & 1.2 & WD repeat domain 47 & WDR47 \\
\hline Hs. 591457 & 1.2 & Polymerase (RNA) III (DNA directed) polypeptide C (62kD) & POLR3C \\
\hline Hs.25527 & 1.2 & Amyloid beta (A4) precursor protein-binding, family A, member 3 (X11-like 2) & АРBA3 \\
\hline Hs.69328 & 1.2 & Lymphocyte antigen 96 & LY96 \\
\hline Hs. 87726 & 1.2 & Golgi associated, gamma adaptin ear containing, ARF binding protein 3 & GGA3 \\
\hline Hs. 595540 & 1.2 & SEC24 related gene family, member A (S. cerevisiae) & SEC24A \\
\hline Hs.151641 & 1.2 & Leucine rich repeat containing 32 & LRRC32 \\
\hline Hs.213389 & 1.2 & Golgi autoantigen, golgin subfamily b, macrogolgin (with transmembrane signal), 1 & GOLGB1 \\
\hline Hs.1481 & 1.2 & Histidine decarboxylase & HDC \\
\hline Hs. 17883 & 1.2 & Protein phosphatase $1 \mathrm{G}$ (formerly $2 \mathrm{C}$ ), magnesium-dependent, gamma isoform & PPM1G \\
\hline Hs.404119 & 1.2 & Tissue specific transplantation antigen $\mathrm{P} 35 \mathrm{~B}$ & TSTA3 \\
\hline Hs.642714 & 1.2 & Interleukin 24 & IL24 \\
\hline Hs.591381 & 1.2 & Insulin receptor & INSR \\
\hline Hs.433419 & 1.2 & Cytochrome c oxidase subunit IV isoform 1 & COX4l1 \\
\hline Hs.153863 & 1.2 & SMAD, mothers against DPP homolog 6 (Drosophila) & SMAD6 \\
\hline Hs.310645 & 1.2 & RAB1A, member RAS oncogene family & RAB1A \\
\hline Hs.492445 & 1.2 & $\begin{array}{l}\text { Transcribed locus, strongly similar to NP_056986.2 progestin-induced protein; ubiquitin-protein ligase; } \\
\text { hyperplastic discs protein homolog; progestin induced protein [Homo sapiens] }\end{array}$ & \\
\hline Hs.509718 & 1.2 & Zinc finger protein 318 & ZNF318 \\
\hline Hs.106511 & 1.2 & Protocadherin 17 & PCDH17 \\
\hline Hs.353035 & 1.2 & TWIST neighbor & TWISTNB \\
\hline Hs.519523 & 1.3 & Serpin peptidase inhibitor, clade B (ovalbumin), member 6 & SERPINB6 \\
\hline Hs. 475334 & 1.3 & KIAA0280 & KIAA0280 \\
\hline Hs.592567 & 1.3 & NA & NA \\
\hline Hs.529846 & 1.3 & Calcium modulating ligand & CAMLG \\
\hline Hs. 462732 & 1.3 & Suppressor of zeste 12 homolog (Drosophila) & SUZ12 \\
\hline Hs.169474 & 1.3 & Eukaryotic translation initiation factor $2 \mathrm{~B}$, subunit 4 delta, $67 \mathrm{kDa}$ & EIF2B4 \\
\hline Hs. 278573 & 1.3 & CD59 molecule, complement regulatory protein & CD59 \\
\hline Hs.510172 & 1.3 & Human immunodeficiency virus type I enhancer binding protein 2 & HIVEP2 \\
\hline Hs. 518460 & 1.3 & Adaptor-related protein complex 2 , mu 1 subunit & AP2M1 \\
\hline Hs.269092 & 1.3 & Chromodomain protein, Y-like & CDYL \\
\hline Hs. 513044 & 1.3 & Chondroitin sulfate proteoglycan 4 (melanoma-associated) & CSPG4 \\
\hline Hs.584782 & 1.3 & Nardilysin (N-arginine dibasic convertase) & NRD1 \\
\hline Hs.473087 & 1.3 & CTP synthase & CTPS \\
\hline
\end{tabular}




\begin{tabular}{|c|c|c|}
\hline Hs.258314 & 1.3 & Brain and reproductive organ-expressed (TNFRSF1A modulator) \\
\hline Hs. 556018 & 1.3 & Interleukin-1 receptor-associated kinase 1 binding protein 1 \\
\hline Hs.31334 & 1.3 & PRP6 pre-mRNA processing factor 6 homolog (S. cerevisiae) \\
\hline Hs.12970 & 1.3 & Proteasome (prosome, macropain) 26S subunit, non-ATPase, 3 \\
\hline Hs. 475150 & 1.3 & Death-inducing-protein \\
\hline Hs. 443465 & 1.3 & Transcription elongation regulator 1 \\
\hline Hs. 502528 & 1.3 & NADH dehydrogenase (ubiquinone) Fe-S protein $3,30 \mathrm{kDa}$ ( $\mathrm{NADH}$-coenzyme $\mathrm{Q}$ reductase) \\
\hline Hs.597390 & 1.3 & Transcribed locus \\
\hline Hs.601814 & 1.3 & Transcribed locus \\
\hline Hs. 122346 & 1.3 & MAD2L1 binding protein \\
\hline Hs. 567380 & 1.3 & Far upstream element (FUSE) binding protein 1 \\
\hline Hs.3439 & 1.3 & Stomatin (EPB72)-like 2 \\
\hline Hs. 517948 & 1.3 & DEAH (Asp-Glu-Ala-His) box polypeptide 30 \\
\hline Hs.75782 & 1.3 & General transcription factor IIIC, polypeptide 2 , beta $110 \mathrm{kDa}$ \\
\hline Hs.22616 & 1.3 & KIAA0664 \\
\hline Hs.3873 & 1.3 & Palmitoyl-protein thioesterase 1 (ceroid-lipofuscinosis, neuronal 1, infantile) \\
\hline Hs.465929 & 1.3 & Calponin 1, basic, smooth muscle \\
\hline Hs. 445350 & 1.3 & Flavin containing monooxygenase 3 \\
\hline Hs. 182625 & 1.3 & VAMP (vesicle-associated membrane protein)-associated protein B and C \\
\hline Hs.202470 & 1.3 & UV radiation resistance associated gene \\
\hline Hs.598003 & 1.3 & Transcribed locus \\
\hline Hs.295917 & 1.3 & ATPase, $\mathrm{H}+$ transporting, lysosomal $56 / 58 \mathrm{kDa}$, V1 subunit B2 \\
\hline Hs.283111 & 1.3 & KIAA0323 \\
\hline Hs.79081 & 1.3 & Protein phosphatase 1 , catalytic subunit, gamma isoform \\
\hline Hs. 528833 & 1.3 & Fatso \\
\hline Hs.201253 & 1.3 & Cytoskeleton associated protein 5 \\
\hline Hs.601349 & 1.3 & Transcribed locus \\
\hline Hs. 591910 & 1.3 & Tripartite motif-containing 32 \\
\hline Hs.467236 & 1.3 & Zinc finger protein 160 \\
\hline Hs.3254 & 1.3 & Mitochondrial ribosomal protein $\mathrm{L} 23$ \\
\hline Hs. 500245 & 1.3 & Discs, large homolog 5 (Drosophila) \\
\hline Hs. 524484 & 1.4 & Integrin, alpha 7 \\
\hline Hs.31387 & 1.4 & Ariadne homolog 2 (Drosophila) \\
\hline Hs. 631618 & 1.4 & Tropomyosin 4 \\
\hline Hs.523238 & 1.4 & Nucleolar and coiled-body phosphoprotein 1 \\
\hline Hs.524899 & 1.4 & Sin3A-associated protein, $18 \mathrm{kDa}$ \\
\hline Hs.69745 & 1.4 & Ferredoxin reductase \\
\hline Hs.26770 & 1.4 & Fatty acid binding protein 7 , brain \\
\hline Hs.2430 & 1.4 & Vacuolar protein sorting 72 (S. cerevisiae) \\
\hline Hs.151777 & 1.4 & Eukaryotic translation initiation factor 2 , subunit 1 alpha, $35 \mathrm{kDa}$ \\
\hline Hs.157394 & 1.4 & Hydroxyacylglutathione hydrolase \\
\hline Hs.269128 & 1.4 & SNF1-like kinase 2 \\
\hline Hs. 642820 & 1.4 & Transcribed locus \\
\hline Hs. 128548 & 1.4 & WD repeat domain 1 \\
\hline Hs. 523215 & 1.4 & NADH dehydrogenase (ubiquinone) 1 beta subcomplex, $8,19 \mathrm{kDa}$ \\
\hline Hs.139896 & 1.4 & Macrophage erythroblast attacher \\
\hline Hs.65234 & 1.4 & DEAD (Asp-Glu-Ala-Asp) box polypeptide 27 \\
\hline Hs. 441926 & 1.4 & Chromosome 13 open reading frame 24 \\
\hline Hs.291212 & 1.4 & Tubulin-specific chaperone a \\
\hline Hs. 523414 & 1.4 & Insulin-like growth factor 2 (somatomedin A) \\
\hline Hs.12107 & 1.4 & Chromatin modifying protein $2 \mathrm{~A}$ \\
\hline Hs.569312 & 1.4 & Dynein, cytoplasmic 1, heavy chain 1 \\
\hline Hs. 564847 & 1.4 & Small nuclear ribonucleoprotein polypeptide $\mathrm{N}$ \\
\hline Hs. 556600 & 1.4 & Myosin, light polypeptide kinase \\
\hline
\end{tabular}

BRE

IRAK1BP1

PRPF6

PSMD3

DIP

TCERG1

NDUFS3

MAD2L1BP

FUBP1

STOML2

DHX30

GTF3C2

KIAA0664

PPT1

CNN1

FMO3

VAPB

UVRAG

ATP6V1B2

KIAA0323

PPP1CC

FTO

CKAP5

TRIM32

ZNF160

MRPL23

DLG5

ITGA7

ARIH2

TPM4

NOLC1

SAP18

FDXR

FABP7

VPS72

EIF2S1

$\mathrm{HAGH}$

SNF1LK2

WDR1

NDUFB8

MAEA

DDX27

C13orf24

TBCA

IGF2

CHMP2A

DYNC1H1

SNRPN

MYLK 


\begin{tabular}{|c|c|c|c|}
\hline Hs.3459 & 1.4 & Ubiquitin-binding protein homolog & UBPH \\
\hline Hs. 463465 & 1.5 & UTP18, small subunit (SSU) processome component, homolog (yeast) & UTP18 \\
\hline Hs. 17441 & 1.5 & Collagen, type IV, alpha 1 & COL4A1 \\
\hline Hs. 514435 & 1.5 & Splicing factor $3 b$, subunit $3,130 \mathrm{kDa}$ & SF3B3 \\
\hline Hs. 190968 & 1.5 & Ribosomal protein S21 & RPS21 \\
\hline Hs.448589 & 1.5 & Ankyrin repeat domain 1 (cardiac muscle) & ANKRD1 \\
\hline Hs.434937 & 1.5 & Peptidylprolyl isomerase B (cyclophilin B) & PPIB \\
\hline Hs.462590 & 1.5 & Myosin XVIIIA & MY018A \\
\hline Hs. 413801 & 1.6 & Proteasome (prosome, macropain) activator subunit 4 & PSME4 \\
\hline Hs.12457 & 1.6 & Nucleoporin $133 \mathrm{kDa}$ & NUP133 \\
\hline Hs.189716 & 1.6 & NADH dehydrogenase (ubiquinone) 1, alpha/beta subcomplex, 1, 8kDa & NDUFAB1 \\
\hline Hs. 602622 & 1.7 & Transcribed locus & \\
\hline Hs.508716 & 2.1 & Collagen, type IV, alpha 2 & COL4A2 \\
\hline Hs. 632144 & 4.9 & Serum amyloid A1 & SAA1 \\
\hline \multicolumn{4}{|l|}{ Mut-3243 } \\
\hline Hs. 527919 & 0.7 & Karyopherin alpha 3 (importin alpha 4) & KPNA3 \\
\hline Hs. 35052 & 0.7 & Testis enhanced gene transcript (BAX inhibitor 1) & TEGT \\
\hline Hs. 481551 & 0.7 & 5-methyltetrahydrofolate-homocysteine methyltransferase reductase & MTRR \\
\hline Hs.432642 & 0.8 & Mitogen-activated protein kinase 12 & MAPK12 \\
\hline Hs. 515003 & 0.8 & Chromosome 19 open reading frame 6 & C19orf6 \\
\hline Hs. 247729 & 0.8 & RAS (RAD and GEM)-like GTP-binding 1 & REM1 \\
\hline Hs.99960 & 0.8 & Membrane-spanning 4-domains, subfamily A, member 3 (hematopoietic cell-specific) & MS4A3 \\
\hline Hs.296949 & 0.9 & Claudin 9 & CLDN9 \\
\hline Hs.159509 & 0.9 & Serpin peptidase inhibitor, clade $F$ (alpha-2 antiplasmin, pigment epithelium derived factor), member 2 & SERPINF2 \\
\hline Hs.32505 & 0.9 & Potassium inwardly-rectifying channel, subfamily J, member 4 & KCNJ4 \\
\hline Hs. 631564 & 0.9 & Protein kinase C, gamma & PRKCG \\
\hline Hs.481466 & 0.9 & Transcribed locus, strongly similar to NP_060610.1 hypothetical protein FLJ10565 [Homo sapiens] & \\
\hline Hs.431792 & 0.9 & Growth arrest-specific 8 & GAS8 \\
\hline Hs.399800 & 0.9 & A kinase (PRKA) anchor protein 8-like & AKAP8L \\
\hline Hs.29282 & 0.9 & Mitogen-activated protein kinase kinase kinase 3 & MAP3K3 \\
\hline Hs.37860 & 0.9 & Kruppel-like factor 1 (erythroid) & KLF1 \\
\hline Hs.631759 & 1.0 & NA & NA \\
\hline Hs. 442182 & 1.1 & ATP-binding cassette, sub-family C (CFTR/MRP), member 6 & ABCC6 \\
\hline Hs.463045 & 1.1 & GCN5 general control of amino-acid synthesis 5-like 2 (yeast) & GCN5L2 \\
\hline Hs. 503546 & 1.1 & Fatty acid desaturase 1 & FADS1 \\
\hline Hs.100921 & 1.1 & Zinc finger protein 193 & ZNF193 \\
\hline Hs.279906 & 1.1 & Cyclin T1 & CCNT1 \\
\hline Hs.179565 & 1.1 & MCM3 minichromosome maintenance deficient 3 (S. cerevisiae) & MCM3 \\
\hline \multirow[t]{2}{*}{ Hs. 584836} & 1.1 & Transcribed locus, strongly similar to NP_004782.1 integrin, beta-like 1 (with EGF-like repeat domains) & \\
\hline & & [Homo sapiens] & \\
\hline Hs.503165 & 1.1 & Centaurin, delta 2 & CENTD2 \\
\hline Hs. 523835 & 1.1 & CDK2-associated protein 2 & CDK2AP2 \\
\hline Hs.119014 & 1.1 & Zinc finger protein 175 & ZNF175 \\
\hline Hs. 97616 & 1.1 & SH3-domain GRB2-like 1 & SH3GL1 \\
\hline Hs.533613 & 1.1 & Troponin T type 2 (cardiac) & TNNT2 \\
\hline Hs. 590970 & 1.1 & AXL receptor tyrosine kinase & AXL \\
\hline Hs. 1570 & 1.1 & Histamine receptor $\mathrm{H} 1$ & $\mathrm{HRH} 1$ \\
\hline Hs. 88556 & 1.1 & Histone deacetylase 1 & HDAC1 \\
\hline Hs.268107 & 1.1 & Multimerin 1 & MMRN1 \\
\hline Hs.166011 & 1.1 & Catenin (cadherin-associated protein), delta 1 & CTNND1 \\
\hline Hs.308340 & 1.1 & Nucleoporin $188 \mathrm{kDa}$ & NUP188 \\
\hline Hs. 517888 & 1.1 & Cartilage associated protein & CRTAP \\
\hline Hs.143288 & 1.1 & Hypothetical protein MGC11271 & MGC11271 \\
\hline Hs. 155040 & 1.1 & Zinc finger protein 217 & ZNF217 \\
\hline Hs.274184 & 1.1 & Transcription factor binding to IGHM enhancer 3 & TFE3 \\
\hline
\end{tabular}




\begin{tabular}{|c|c|c|c|}
\hline Hs.502872 & 1.1 & Mitogen-activated protein kinase kinase kinase 11 & MAP3K11 \\
\hline Hs. 611901 & 1.1 & NA & NA \\
\hline Hs. 555936 & 1.1 & APEX nuclease (apurinic/apyrimidinic endonuclease) 2 & APEX2 \\
\hline Hs. 524518 & 1.2 & Signal transducer and activator of transcription 6 , interleukin-4 induced & STAT6 \\
\hline Hs. 632219 & 1.2 & Small optic lobes homolog (Drosophila) & SOLH \\
\hline Hs. 467898 & 1.2 & Adenylate cyclase 3 & $\mathrm{ADCY} 3$ \\
\hline Hs.312098 & 1.2 & ADAM metallopeptidase domain 15 (metargidin) & ADAM15 \\
\hline Hs. 103834 & 1.2 & Transmembrane protein $106 \mathrm{C}$ & TMEM106C \\
\hline Hs.596164 & 1.2 & Transcribed locus & \\
\hline Hs.596205 & 1.2 & Transcribed locus & \\
\hline Hs.283844 & 1.2 & Sideroflexin 3 & SFXN3 \\
\hline Hs. 433445 & 1.2 & Jagged 2 & JAG2 \\
\hline Hs.292843 & 1.2 & Prostaglandin F receptor (FP) & PTGFR \\
\hline Hs. 507515 & 1.2 & Olfactomedin-like 2B & OLFML2B \\
\hline Hs.169998 & 1.2 & Bone marrow stromal cell antigen 1 & BST1 \\
\hline Hs. 430551 & 1.2 & IQ motif containing GTPase activating protein 1 & IQGAP1 \\
\hline Hs.371617 & 1.2 & Polycomb group ring finger 2 & PCGF2 \\
\hline Hs. 611934 & 1.2 & Transcribed locus & \\
\hline Hs.434888 & 1.2 & Ring finger protein 44 & RNF44 \\
\hline Hs.118631 & 1.2 & Timeless homolog (Drosophila) & TIMELESS \\
\hline Hs. 516790 & 1.2 & $\begin{array}{l}\text { Transcribed locus, strongly similar to NP_004714.2 rho/rac guanine nucleotide exchange factor 2; } \\
\text { proliferating cell nucleolar antigen p } 40 \text { [Homo sapiens] }\end{array}$ & \\
\hline Hs.271940 & 1.2 & E74-like factor 4 (ets domain transcription factor) & ELF4 \\
\hline Hs. 483635 & 1.2 & Fibroblast growth factor 1 (acidic) & FGF1 \\
\hline Hs. 85539 & 1.2 & ATP synthase, $\mathrm{H}+$ transporting, mitochondrial F0 complex, subunit $\mathrm{E}$ & ATP5I \\
\hline Hs. 472213 & 1.2 & Ribosome binding protein 1 homolog $180 \mathrm{kDa}(\mathrm{dog})$ & RRBP1 \\
\hline Hs.89643 & 1.2 & Transketolase (Wernicke-Korsakoff syndrome) & TKT \\
\hline Hs.513463 & 1.2 & P8 protein (candidate of metastasis 1 ) & NUPR1 \\
\hline Hs.3109 & 1.2 & Rho GTPase activating protein 4 & ARHGAP4 \\
\hline Hs. 509067 & 1.2 & Platelet-derived growth factor receptor, beta polypeptide & PDGFRB \\
\hline Hs.16355 & 1.2 & Myosin, heavy polypeptide 10, non-muscle & MYH10 \\
\hline Hs.153357 & 1.2 & Procollagen-lysine, 2-oxoglutarate 5-dioxygenase 3 & PLOD3 \\
\hline Hs. 529044 & 1.2 & RAB22A, member RAS oncogene family & RAB22A \\
\hline Hs. 642644 & 1.2 & NA & NA \\
\hline Hs.75189 & 1.2 & Death-associated protein & DAP \\
\hline Hs.213861 & 1.2 & Laminin, alpha 4 & LAMA4 \\
\hline Hs.436298 & 1.2 & Epithelial membrane protein 1 & EMP1 \\
\hline Hs. 462214 & 1.2 & Growth arrest-specific 7 & GAS7 \\
\hline Hs. 155919 & 1.3 & Protein tyrosine phosphatase, receptor type, $\mathrm{K}$ & PTPRK \\
\hline Hs.489284 & 1.3 & Actin related protein $2 / 3$ complex, subunit $1 \mathrm{~B}, 41 \mathrm{kDa}$ & ARPC1B \\
\hline Hs.490273 & 1.3 & CAMP responsive element binding protein 3 -like 2 & CREB3L2 \\
\hline Hs.441783 & 1.3 & Chromosome 14 open reading frame 78 & C14orf78 \\
\hline Hs.438823 & 1.3 & NA & NA \\
\hline Hs.377090 & 1.3 & Zinc fingers and homeoboxes 2 & $\mathrm{ZHX} 2$ \\
\hline Hs.511952 & 1.3 & NA & NA \\
\hline Hs.592129 & 1.3 & Cancer susceptibility candidate 3 & CASC3 \\
\hline Hs.520102 & 1.3 & KIAA0082 & KIAA0082 \\
\hline Hs.501012 & 1.3 & Adducin 3 (gamma) & ADD3 \\
\hline Hs.444767 & 1.3 & Kinesin family member 13B & $\mathrm{KIF} 13 \mathrm{~B}$ \\
\hline Hs. 439312 & 1.3 & Phospholipid transfer protein & PLTP \\
\hline Hs.144492 & 1.3 & Phospholipase C, epsilon 1 & PLCE1 \\
\hline Hs.520740 & 1.3 & Secernin 1 & SCRN1 \\
\hline Hs. 274313 & 1.3 & Insulin-like growth factor binding protein 6 & IGFBP6 \\
\hline Hs.631504 & 1.3 & Ras association (RalGDS/AF-6) domain family 2 & RASSF2 \\
\hline Hs.519909 & 1.3 & Myristoylated alanine-rich protein kinase $\mathrm{C}$ substrate & MARCKS \\
\hline
\end{tabular}




\begin{tabular}{|c|c|c|c|}
\hline Hs.439463 & 1.3 & AE binding protein 1 & AEBP1 \\
\hline Hs.12967 & 1.4 & Spectrin repeat containing, nuclear envelope 1 & SYNE1 \\
\hline Hs.198862 & 1.4 & Fibulin 2 & FBLN2 \\
\hline Hs.433222 & 1.4 & Niemann-Pick disease, type C2 & NPC2 \\
\hline Hs.502461 & 1.4 & Diacylglycerol kinase, zeta $104 \mathrm{kDa}$ & DGKZ \\
\hline Hs.642891 & 1.4 & Transcribed locus & \\
\hline Hs.117060 & 1.4 & Extracellular matrix protein 2 , female organ and adipocyte specific & ECM2 \\
\hline Hs.476092 & 1.4 & C-type lectin domain family 3 , member B & CLEC3B \\
\hline Hs. 507755 & 1.4 & Doublecortin and CaM kinase-like 1 & DCAMKL1 \\
\hline Hs.536663 & 1.4 & Integrin, beta 5 & ITGB5 \\
\hline Hs.520182 & 1.4 & Translocation associated membrane protein 2 & TRAM2 \\
\hline Hs.79299 & 1.4 & Lipoma HMGIC fusion partner-like 2 & LHFPL2 \\
\hline Hs.12956 & 1.4 & Tax1 (human T-cell leukemia virus type I) binding protein 3 & TAX1BP3 \\
\hline Hs.406300 & 1.5 & Ribosomal protein L23 & RPL23 \\
\hline Hs.9754 & 1.5 & Activating transcription factor 5 & ATF5 \\
\hline Hs. 501280 & 1.5 & HtrA serine peptidase 1 & HTRA1 \\
\hline Hs.502756 & 1.5 & AHNAK nucleoprotein (desmoyokin) & AHNAK \\
\hline Hs.153381 & 1.5 & Duffy blood group, chemokine receptor & DARC \\
\hline Hs.143873 & 1.6 & S100 calcium binding protein A10 (annexin II ligand, calpactin I, light polypeptide (p11)) & S100A10 \\
\hline Hs.442378 & 1.6 & Cysteine dioxygenase, type I & CD01 \\
\hline Hs.201641 & 1.6 & Brain abundant, membrane attached signal protein 1 & BASP1 \\
\hline Hs.633826 & 1.6 & Transcribed locus & \\
\hline Hs.531081 & 1.6 & Lectin, galactoside-binding, soluble, 3 (galectin 3) & LGALS3 \\
\hline Hs.391561 & 1.7 & Fatty acid binding protein 4 , adipocyte & FABP4 \\
\hline Hs. 155597 & 1.7 & Complement factor D (adipsin) & CFD \\
\hline Hs.593123 & 1.7 & Transcribed locus & \\
\hline Hs.494173 & 1.7 & Annexin A1 & ANXA1 \\
\hline Hs.598135 & 1.8 & Transcribed locus & \\
\hline Hs.500572 & 1.8 & Fer-1-like 3, myoferlin (C. elegans) & FER1L3 \\
\hline Hs.192233 & 1.9 & Periplakin & PPL \\
\hline Hs.384598 & 1.9 & Serpin peptidase inhibitor, clade G (C1 inhibitor), member 1, (angioedema, hereditary) & SERPING1 \\
\hline Hs.213394 & 2.0 & Erythrocyte membrane protein band 4.1-like 3 & EPB41L3 \\
\hline Hs.436657 & 2.1 & Clusterin & CLU \\
\hline Hs.567497 & 2.1 & Complement component $1, \mathrm{r}$ subcomponent & C1R \\
\hline Hs.533317 & 2.7 & Vimentin & VIM \\
\hline
\end{tabular}





\section{CHAPTER 5}

\section{Increasing levels of the m.9176T $>\mathrm{C}$ mutation in monoclonal human skin fibroblasts induces changes in apoptosis and cytoskeleton organisation}

R.G.E. van Eijsden ${ }^{1,2,3 \$}$, F.H.J. van Tienen ${ }^{1,4}$, L.M.T. Eijssen ${ }^{1,5 \$}$, H.J.M. Smeets ${ }^{1,2}$

${ }^{1}$ Department Genetics and Cell Biology - Clinical Genetics, Maastricht University, Maastricht - The Netherlands.

${ }^{2}$ Research Institute Growth \& Development, Maastricht University, Maastricht - The Netherlands.

${ }^{3}$ MicroArray Facility, VIB, Leuven - Belgium.

${ }^{4}$ Research Institute NUTRIM, Maastricht University, Maastricht - The Netherlands.

${ }^{5}$ BiGCaT Bioinformatics, Maastricht University, Maastricht - The Netherlands.

${ }^{\$}$ Current affiliation.

In preparation 


\begin{abstract}
Heteroplasmic mitochondrial DNA mutations have a threshold of expression above which clinical symptoms become manifest. This threshold is tissue-, mutation- and individual specific and usually the phenotypic expression or transcription profiles of different patients with different mutation loads are being compared to elucidate the underlying pathogenic mechanisms. To reduce sources of variation, which trouble the interpretation of these data, we performed - as a model experiment - gene expression profiling in isolated individual fibroblasts of a single patient with different levels of the m.9176T $>C$ mutation. The m.9176T $>C$ mutation in the adenosine triphosphate synthase 6 (ATP6) gene causes Leigh syndrome when exceeding a mutation load threshold of about 90\%. Furthermore, the mutation load of this mutation is fairly constant among the different tissues analyzed. Fibroblasts were cultured from monoclonal cell populations with mutation loads above and under the level of clinical expression under forced aerobic culturing conditions in order to elicit a pathogenic effect, if present. Gene expression profiles were determined and the most prominent changes appeared to be related to apoptosis and cytoskeleton organisation as an apoptosis early event. Our data show that our model is accurate to study different mtDNA mutation levels in a homogeneous genetic background.
\end{abstract}

\title{
Introduction
}

A characteristic of mitochondrial DNA (mtDNA) mutations is the coexistence of mutated and wildtype mtDNA molecules within mitochondria, cells and tissues. This phenomenon of heteroplasmy partly explains the differences in severity and nature of clinical manifestations of oxidative phosphorylation (OXPHOS) disease, although it is no clear-cut and direct linear relation. The presentation of the m.3243A $>C$ mutation, which is one of the most frequent mtDNA mutations and a major cause of the MELAS syndrome (Myopathy, Encephalopathy, Lactic Acidosis and Stroke-like episodes), is variable and only partially related to the mutation load, suggesting a role for modifying environmental or other genetic factors [99]. The m.8993 $>C / G$ mutation in the adenosine triphosphate synthase 6 (ATP6) gene is an example of an mtDNA mutation with a more direct relation between heteroplasmy level and clinical symptoms. Clinical symptoms usually occur when the mutation load rises above $60 \%$ for the $\mathrm{m} .8993 \mathrm{~A}>\mathrm{G}$ or above $80 \%$ for the m.8993T>C mutation, resulting in Neuropathy, Ataxia, and Retinitis Pigmentosa (NARP) [11]. When mutation loads exceed $90 \%$ subjects present with the severe phenotype of maternally inherited Leigh syndrome [9-11]. A similar correlation between clinical features and mtDNA mutation load most likely exists for the m.9176T>C mutation in the ATP6 gene, although the number of patients and families studied is still small. When the mutation load is higher than the threshold of $90 \%$, subjects present with Leigh syndrome. Mutation loads below the threshold value of $90 \%$ most often results in very mild or even no clinical symptoms at all [3-5, 133-137]. 
To study the molecular biological processes induced by different mtDNA mutation levels by gene expression profiling, in general, tissues or cell lines from different patients with different levels of the mtDNA mutation are being compared. A disadvantage of this approach is the difference in nuclear backgrounds, introducing experimental variability. To circumvent this, cell culture systems can be used where mitochondria from patient cells are transferred to a cell line lacking mtDNA (rho ${ }^{\circ}$ cells) $[138,139]$, allowing the study of mutation loads ranging from $0 \%$ to $100 \% . A$ disadvantage is that rho ${ }^{\circ}$ cells are in general tumour cells with an unstable nuclear background and that an experimental intervention is required to create cell lines with different mutation loads. This might cause unwanted experimental variability and affect gene expression levels. Therefore we established a new model system and analyzed gene expression profiles of cell cultures derived from individual primary skin fibroblasts from a single carrier of the m.9176T>C mutation. Each fibroblast has a different mutation load ranging from $0 \%$ to $100 \%$, providing the opportunity to generate cell lines with different mutation levels of the m.9176T $>C$ point mutation without introducing individual or experimental variability. Cells were cultured under forced aerobic conditions in order to elicit a pathogenic effect, if present.

\section{Materials \& methods}

Cell cultures

A cell culture was setup using fibroblast cells from a female carrier of the m.9176T $>C$ mutation in the ATP6 gene. The mutation load in this cell line was $77.7 \%$. Cells were initially cultured in DMEM with a high glucose concentration ( $4500 \mathrm{mg} / \mathrm{L}$ ) (Invitrogen, Carlsbad, CA, USA) supplemented with 10\% foetal bovine serum (Invitrogen, Carlsbad, CA, USA) and $50 \mu \mathrm{g} / \mathrm{mL}(0.2 \mathrm{mM}$ ) uridine (Acros Organics, Geel, Belgium) in a T75 cell culture flask. After reaching $~ 90 \%$ confluence, cells were washed with $5 \mathrm{~mL}$ Hanks Balanced Salt Solution (HBSS) (Invitrogen, Carlsbad, CA), treated with trypLE (Invitrogen, Carlsbad, CA, USA) to detach them from the bottom of the cell culture flask, and resuspended in culture medium to a final concentration of 40 cells $/ \mathrm{mL}$ of which $1 \mathrm{~mL}$ was transferred to each well of two 6-well culture plates. Conditional medium was prepared from a wild-type fibroblast control cell culture by sterilizing 2 day old medium from a $70 \%$ confluent culture through a $0.2 \mu \mathrm{m}$ filter. Next, $1 \mathrm{~mL}$ conditional medium was added to each well. Each 4-5 days, $1 \mathrm{~mL}$ fresh culture medium was added to each well. After two weeks, when colonies of cells were formed in each well, medium was removed and cells were washed with $1 \mathrm{~mL}$ HBSS. Cells were treated with $100 \mu \mathrm{L}$ of trypLE for 5 minutes at $37^{\circ} \mathrm{C}$ to detach them from the bottom of the cell culture plate. From each well, one colony was manually transferred by pipette to a new well in a 6 well culture plate containing a mixture of $1 \mathrm{~mL}$ culture medium and $1 \mathrm{~mL}$ conditional medium. Every 4-5 days, $1 \mathrm{~mL}$ culture medium was added or the medium was refreshed with $2 \mathrm{~mL}$ culture medium. When a well reached $90 \%$ confluence, cells were treated with trypLE and one half was transferred to a new T75 culture flask containing $12 \mathrm{~mL}$ culture medium. The other half of the cell pellet was resuspended in $50 \mu \mathrm{L} 1 \mathrm{x}$ phosphatebuffered Saline (PBS) $+1 \%$ polyvinylpyrolidone + 
phenol red to use for later mutation load quantification (time point 1). When the cell culture reached 50\% confluence, medium was removed and cells were washed with $5 \mathrm{~mL} P B S$ and $12 \mathrm{~mL}$ stringent medium was added. The stringent medium contained DMEM without glucose or sodium pyruvate (Invitrogen, Carlsbad, CA, USA), supplemented with $20 \%$ dialyzed foetal bovine serum (Invitrogen, Carlsbad, CA, USA), $5 \mathrm{mM}$ galactose (Sigma-Aldrich, St. Louis, MO, USA), and 50 $\mu \mathrm{g} / \mathrm{mL}(0.2 \mathrm{mM}$ ) uridine. Omitting glucose and sodium pyruvate from the medium forces the cells to use the OXPHOS system (aerobic energy metabolism). After 72 hours on the stringent medium, RNA and DNA (time point 2) were isolated from the cell cultures.

\section{RNA and DNA isolation from fibroblasts}

Total RNA was isolated from the fibroblast cell cultures according to the manufacturer's protocol using TRIzol reagent (Invitrogen, Carlsbad, CA, USA) and RNA was purified using the RNeasy clean-up kit (Qiagen, Hilden, Germany). RNA quantity and purity were determined spectrophotometrically with the Nanodrop ND-1000 (Nanodrop Technologies, Wilmington, DE, USA) and RNA integrity was assessed by determining the RNA $28 \mathrm{~S} / 18 \mathrm{~S}$ ratio on a Bioanalyser 2100 (Agilent Technologies, Santa Clara, CA, USA).

DNA was isolated from the remaining interphase and organic phase from the TRIzol RNA isolation using a back extraction buffer. The back extraction buffer contained $4 \mathrm{M}$ guanidine thiocyanate (Sigma-Aldrich, St. Louis, MO, USA), 50 mM sodiumcitrate (Merck \& Co., Inc., Whitehouse Station, NJ, USA), and $1 \mathrm{M}$ Tris (free base) (Roche, Basel, Switzerland). Back extraction buffer ( $500 \mu \mathrm{L}$ per $\mathrm{mL}$ TRIzol) was added to the samples and the samples were vortexed for 15 seconds. Samples were incubated for 10 minutes at room temperature and centrifuged at maximum speed in an eppendorf centrifuge. Supernatant was removed and the DNA pellet was washed twice with $75 \%$ ethanol $(750 \mu \mathrm{L}$ per $\mathrm{mL}$ TRIzol). DNA pellets were air dried and dissolved in $30 \mu \mathrm{L}$ nuclease free water. DNA purity and concentration were determined spectrophotometrically using the Nanodrop ND-1000.

\section{m.9176T>C mutation load quantification}

Forward (5'-TCATGCACCTAATTGGAAGCG-3') and reverse (5'-GTGTTGTCGTGCAGGTACCAGCTTACT-3') primers were used for amplification of the fragment carrying the m.9176T $>C$ mutation by polymerase chain reaction (PCR). The reverse primer contained a three nucleotide mismatch at positions 18-20 of the primer sequence to create a restriction site in the PCR fragment for the BstXI restriction enzyme, if the mutation is present. Cycling conditions were as follows: denaturation for $6^{\prime}$ at $94^{\circ} \mathrm{C}, 35$ cycles of $1^{\prime}$ at $92^{\circ} \mathrm{C}, 1^{\prime}$ at $52^{\circ} \mathrm{C}$, and $45^{\prime \prime}$ at $72^{\circ} \mathrm{C}$, and a final elongation step for $7^{\prime}$ at $72^{\circ} \mathrm{C}$. A FAM-labelled forward primer was added to the final PCR cycle with a denaturation step for $2^{\prime}$ at $94^{\circ} \mathrm{C}$, annealing for $1^{\prime} 52^{\circ} \mathrm{C}$, and an elongation step for 7 at $72^{\circ} \mathrm{C}$. $\mathrm{PCR}$ was carried out directly on $2 \mu \mathrm{L}$ of the cell suspension stored previously (time point 1 - see above) after a cell lyses step, or on $2 \mu \mathrm{L}$ DNA solution from the back extraction isolation (time point 2 - 
see above). Cell lyses solution was prepared by dissolving $4.05 \mathrm{mg}$ Dithiothreitol (DTT) in $210 \mu \mathrm{L}$ $\mathrm{NaOH}(1 \mathrm{M})$ and $210 \mu \mathrm{L}$ sterile water. Before use, the lyses solution was UV-irradiated for 1 hour to prevent DNA contamination. One $\mu \mathrm{L}$ lyses solution was added to $2 \mu \mathrm{L}$ of the cell suspension. Lyses was performed at $65^{\circ} \mathrm{C}$ for 10 minutes.

Heteroplasmy levels were determined by digestion with the BstXI enzyme (New England Biolabs, Ipswich - MA, USA) resulting in a wild-type fragment of $169 \mathrm{bp}$ and mutant fragments of 148 and $21 \mathrm{bp}$. Digested products were analyzed on an ABI-PRISM 3100 genetic analyzer using the GeneScan Analysis 3.7 software package (Applied Biosystems, Foster City, CA, USA). The ratio of the area under the $148 \mathrm{bp}$ mutant peak and the sum of the area's under the mutant (148 bp) and wild-type (169 bp) peaks was calculated to determine the heteroplasmy levels of the m.9176T>C mutation.

\section{Gene expression profiling}

One $\mu \mathrm{g}$ of total RNA from each sample was amplified using the Amino Allyl MessageAmp II aRNA kit (Ambion, Austin, TX, USA) to generate amino allyl aRNA (AA-aRNA), according to the manufacturer's protocol. A UTP: AA-UTP ratio of 7:3 was used instead of 1:1. The size distribution profile of the aRNA was assessed using a Bioanalyser 2100 (Agilent Technologies, Santa Clara, CA, USA). Each amino AA-aRNA sample was coupled to Cy3 or Cy5 dye (Amersham Biosciences, Uppsala, Sweden) and cleaned up with the RNeasy Minelute clean-up kit (Qiagen, Hilden, Germany). RNA quantity and dye incorporation efficiency were determined spectrophotometrically using the Nanodrop ND-1000. Hybridisation was performed on CodeLink slides (Amersham Biosciences, Uppsale - Sweden) containing the Operon Human oligo collection V2 with 21521 features (Operon Biotechnologies, Inc., Huntsville, AL, USA) printed by the UMC Microarray Facility (University Medical Centre Utrecht, Utrecht, The Netherlands). Slides were blocked in 5xSSC, 25\% Formamide, 0.1\% SDS, 1\% BSA (Sigma-Aldrich, St. Louis, MO, USA) for 1 hour. Fragmentation was performed on 120 pmol of each dye-labelled aRNA sample ( $2 \%-4.5 \%$ dye incorporation) using RNA fragmentation buffer (Ambion, Austin, TX, USA), mixed with $100 \mu \mathrm{g}$ tRNA (Roche, Basel, Switzerland) and $50 \mu \mathrm{g}$ Herring sperm DNA (Invitrogen, Carlsbad, CA, USA) in $120 \mu \mathrm{L}$ hybridisation mix ( $5 \times \mathrm{SSC}$, 25\% Formamide, $0.1 \% \mathrm{SDS}$ ), denaturated for 5 minutes at $95^{\circ} \mathrm{C}$, hybridised for 16 hours at $42^{\circ} \mathrm{C}$ and washed 3 times with decreasing concentrations of SSC and SDS (1xSSC, $0.2 \%$ SDS, $0.1 \times S S C, 0.2 \% S D S$, and $0.1 \times S S C$ respectively). Hybridisation, washing, and slide drying were performed on a HS 4800 hybridisation station (Tecan, Mannedorf/Zurich, Switzerland). Hybridisation was carried out according to the loop design illustrated in figure 1. Slides were scanned on a GenePix 4000B scanner (Molecular Devices, Sunnyvale, CA, USA) with laser power setting at 100\% for both channels and photo multiplier settings at 625 and 746 for the green and the red channel respectively, which ensured a maximum of $0.05 \%$ of all spots to be saturated. Scanned images were analyzed and spots were quantified using Array-Pro analyzer software (Media Cybernetics, Bethesda, MD, USA). Bad spots were flagged manually. 


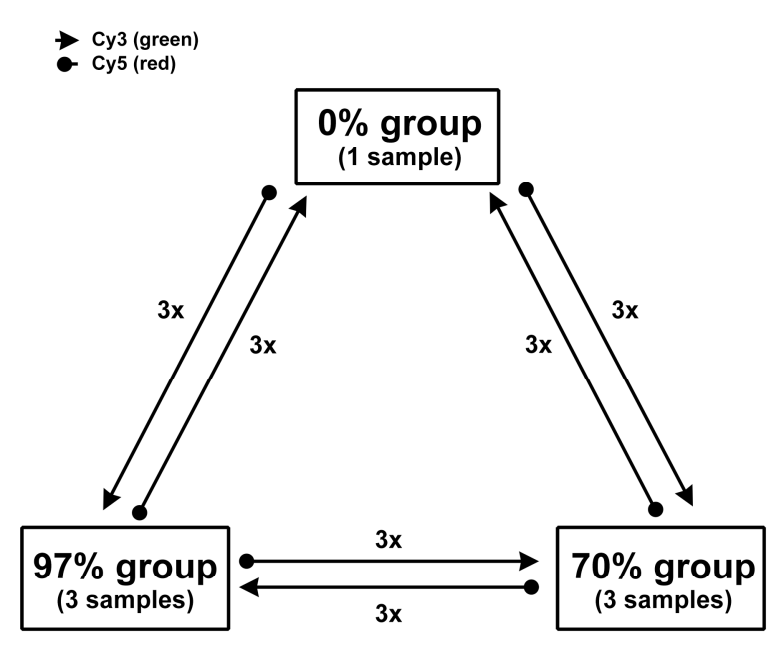

Figure 1: Microarray experimental design.

0\% group: fibroblast cell line with $0 \%$ mutation load; $70 \%$ group: three fibroblast cell lines with mutation loads between 65\% and 75\%; 97\% group: two fibroblast cell lines with mutation loads between $95 \%$ and $100 \%$. Each sample was used four times for hybridisation because a dye-swap was carried out, except for the one sample in group 1 which was used 12 times.

Each arrow indicates a two colour hybridisation on a microarray slide, where the head of the arrow indicates a cy3-labeled samples and the tail of the arrow indicates a cy5-labeled sample.

Microarray data analysis and data mining

$\mathrm{R}$, a free software environment for statistical computing and graphics, was used with the LIMMA library to perform the microarray data analysis [40, 140]. Background correction was carried out using the method "half", "print tip loes" was used as within array normalisation method, and the "scale" method was used for between array normalisation [141]. In the LIMMA procedure a correction was applied for the dye-effect and the dye-swaps were taken into account as technical replicates [142]. Before model fitting, data from all spots with foreground intensities smaller than twice the background intensities in all samples and in both colours were removed from the dataset. Since there was only one sample in group 1 (see figure 1) resulting in a small within group variance, it was likely that with standard stringency criteria ( $p$-value<0.05) a lot of transcripts would be indicated as significantly differentially expressed of which the majority would be false positive. To overcome this and to correct for multiple testing, the restriction criteria were stringently adjusted and transcripts with an adjusted (Benjamini \& Hochberg) p-value $<0.001$ and an absolute fold-change $>$ 2-fold were selected as significantly differentially expressed [143]. To identify biological processes that were enriched for differentially expressed genes were involved, a process based analysis was carried out using the web-based Database for Annotation, Visualisation and Integrated Discovery (DAVID) [76].

\section{Results and Discussion}

\section{Cell cultures and m.9176T>C mutation loads}

Culturing single fibroblasts of a female carrier of the m.9176T $>C$ mutation resulted in six cell cultures with different mutation loads and the same nuclear background. The remaining cell cultures grew very slowly or stopped growing for unknown reasons. The cultures could be sub- 
divided into three groups based on their mutation loads. The first group comprising one sample with a mutation load of $0 \%$, a second group containing three samples with mutation loads between $65 \%$ and $75 \%$, and a third group of two samples with mutation loads between $95 \%$ and $100 \%$ (see table 1). Cells were cultured for three days under aerobic conditions, using galactose medium, to force them to use the OXPHOS system for their energy demand, after which RNA was isolated. Mutation loads remained more or less the same during these three days (table 1). The largest difference between time point one and two was $8 \%$ for cell culture B5. However, this did not have consequences for the mutation load group classification. There was no mutation load difference for cell culture A1 for which the mutation load remained $0 \%$.

Table 1: Overview of cell cultures from the cell culture experiment as described in the materials and methods section

\begin{tabular}{lllll}
\hline Cell culture & $\begin{array}{l}\text { Mutation load at } \\
\text { time point 1 }\end{array}$ & $\begin{array}{l}\text { Mutation load at } \\
\text { time point 2 }\end{array}$ & Group ID & Group description \\
\hline A1 & $0,0 \%$ & $0,0 \%$ & 1 & $0 \%$ \\
\hline A6 & $67,7 \%$ & $65,6 \%$ & 2 & $65-75 \%$ \\
B6 & $68,3 \%$ & $69,7 \%$ & 2 & \\
B5 & 67,15 & $75,2 \%$ & 2 & $95-100 \%$ \\
\hline A4 & $96,6 \%$ & $97,3 \%$ & 3 & 3 \\
B2 & $96,5 \%$ & $100 \%$ & 3 & \\
\hline
\end{tabular}

Cell culture $\mathrm{A} 1$ had an m.9176T>C mutation load of $0 \%$. Cell cultures A6, B5, and B6 had mutation loads between $65 \%$ and $75 \%$. Cell cultures $A_{4}$ and $B 2$ had mutation loads between $95 \%$ and $100 \%$. Time point 1: just before applying the stringent galactose medium. Time point 2: After 72 hours on stringent galactose medium.

These cell lines are only theoretically monoclonal, because it is not completely certain that a single colony originates from a single cell, but the distribution of mutation levels will be much more confined to the mean than in the original fibroblast culture which shows a broad distribution with high and low mutation levels (carrier 3, chapter 4, figure 2 in [144]). Analysis of single cells from these cell lines could reveal the distribution pattern. Our approach is superior to the use of cybrid systems to study the effects of differences in mutation loads $[138,139]$ as cybrids are in general tumour cells with an unstable nuclear background, which introduces unwanted variability in gene expression experiments. This may be less of a disadvantage when studying specific processes, but makes them rather unsuitable for genome-wide gene expression profiling. The use of cell lines from different patients with different mutation loads of the m.9176T $>C$ mutation has the disadvantage of differences in nuclear genetic background, which may mask the effect of the mutation load difference. From previous studies we know that the gene expression differences in fibroblasts with OXPHOS deficiency can be subtle. Our new approach of making 'monoclonal' cell lines allowed us to investigate gene expression differences in cultured skin fibroblast carrying different mutation loads of the m.9176T $>\mathrm{C}$ mutation with the same nuclear genetic background and minimal experimental and genetic variation. This approach might also be suitable to study heteroplasmy effects for other mtDNA mutations. However, this will depend on whether or not 
the mutation is present in fibroblast cells at different levels and on the distribution and the stability of the mutation loads in individual cells.

Gene expression differences between fibroblasts with different m.9176T >C mutation loads

Microarray gene expression experiments were carried out using RNA from the three cell culture groups according to the experimental design depicted in figure 1. After removal of control spots and spots which did not reach a signal intensity higher than twice the background intensity, 10413 data points remained. For some transcripts more than one probe was spotted. This resulted in replicate entries within the list of significantly differentially expressed genes after LIMMA analysis. For the group 70\% versus group 0\% (70vso) comparison, 882 unique transcripts were differentially expressed with 289 and 593 transcripts up- and down-regulated, respectively (supplementary table S1). For the group 97\% versus group 0\% (97vso) comparison, 914 transcripts were differentially expressed, of which 365 and 549 were up- and down-regulated respectively (supplementary table S2). In the group 97\% versus group 70\% (97vs70) comparison 249 transcripts were differentially expressed with 135 up- and 114 down-regulated (supplementary table S3). Table 2 and the Venn diagram in figure 2 summarise the number of differentially expressed genes and illustrate that most gene expression changes occurred in the comparisons with group $0 \%$ Moreover, there was a large overlap between the differentially expressed transcripts in the comparisons with group $0 \%$. A number of 520 transcripts corresponding with a percentage of $59 \%$ (520/882) and 57\% (520/914) of the differentially expressed genes for respectively the 70vso and 97vso comparisons overlapped.

Table 2: Significantly differentially expressed genes

\begin{tabular}{|c|c|c|c|c|c|}
\hline \multicolumn{6}{|c|}{ Group 70\% versus Group 0\% } \\
\hline all-total & all-up & all-down & total-unique & up-unique & down-unique \\
\hline 910 & 301 & 609 & 882 & 289 & 593 \\
\hline \multicolumn{6}{|c|}{ Group $97 \%$ versus Group 0\% } \\
\hline all-total & all-up & all-down & total-unique & up-unique & down-unique \\
\hline 937 & 372 & 665 & 914 & 365 & 549 \\
\hline \multicolumn{6}{|c|}{ Group $97 \%$ versus Group 70\% } \\
\hline all-total & all-up & all-down & total-unique & up-unique & down-unique \\
\hline 249 & 135 & 114 & 249 & 135 & 114 \\
\hline
\end{tabular}

Significantly differentially expressed genes with an adjusted p-value<0.001 (Benjamini \& Hochberg) and a minimum absolute foldchange $>2$. For a number of transcripts, more than one probe was spotted on the array, resulting in replicate entries in the differentially expressed gene lists. Here, the overall number of entries and the unique number of entries are listed. 


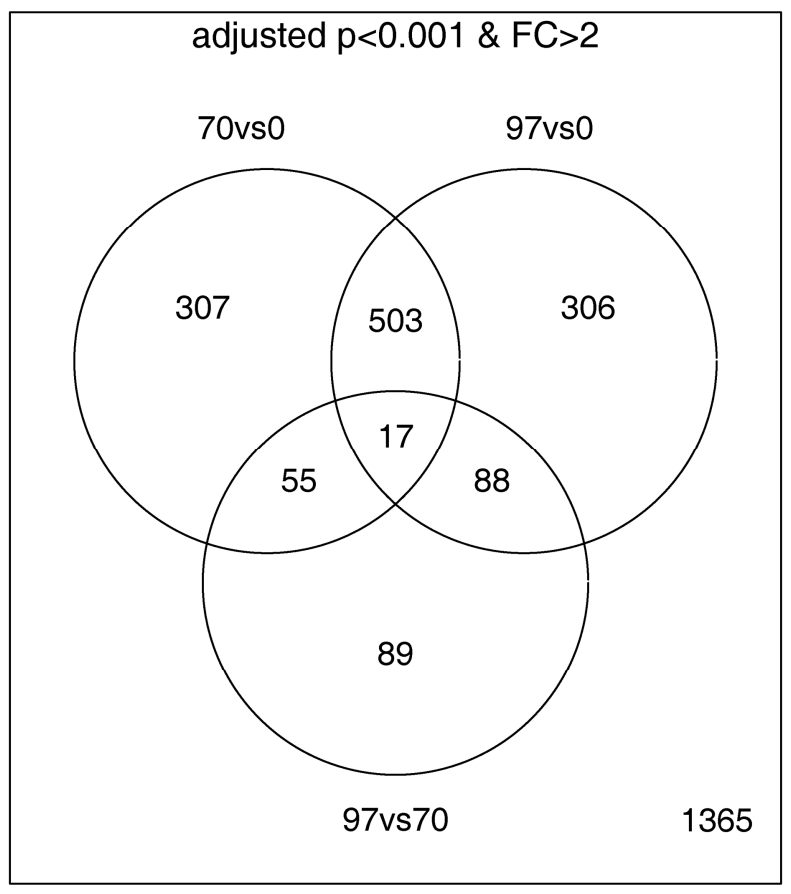

Figure 2: Venn diagram of the results from all three comparisons

Results in the Venn diagram are based on the unique genes in the differentially expressed gene lists only. The highest number of differentially expressed genes was found in the comparisons with group $0 \%$. There is a large overlap between the differentially expressed genes in comparisons between group $70 \%$ and $0 \%$, and group $97 \%$ and $0 \%$. p: p-value; FC: fold-change

The quality of the microarray experiment and data analysis is shown by the consistent appearance of genes which were present multiple times on the microarray and appear multiple times in the lists of differentially expressed genes. That most gene expression changes with substantial overlap occur in the comparisons with group $0 \%$ is expectable, since these comparisons correspond to the contrasting wild-type (no mutation) versus a fairly high mutation (>70\% mutation load) situation. Another factor which might have contributed to the higher number of differentially expressed genes could be that there was only one sample in group $0 \%$, causing the within group variance in this group to be smaller than in the other groups. This was taken into account by increasing the stringency criteria for a gene to be differentially expressed only when the adjusted p-value (corrected for multiple testing) was $<0.001$, in contrast to the often applied adjusted p-value cut-off of 0.05 , and when the absolute fold-change was $>2$-fold. The processes in the 7ovso comparison could be the first processes elicited by the genetic defect, but could also reflect rescue processes as clinical manifestations do not occur at that level. Additionally, the $97 \mathrm{vso}$ and $97 \mathrm{vs70}$ comparisons could identify processes directly related to the pathophysiology or failing of the rescue process and involved in the transition from non-symptomatic to symptomatic disease. However, it has to be kept in mind that symptoms do not occur in fibroblasts and that the processes in the affected tissues may differ.

Process based analysis comparison group 70\% versus group 0\%

DAVID functional annotation clustering of the differentially expressed genes from the 70vso comparison resulted in six gene clusters with at least one $\mathrm{GO} \mathrm{BP}_{5}$ term having a p-value $<0.05$ (table 3). The first cluster contained genes with functions involved in programmed cell death. The cluster contained 48 unique transcripts (table 4) of which 40 were down-regulated and only 8 were up-regulated. Gene expression changes having a stimulatory effect on apoptosis were 
significant down-regulations of apoptosis inhibitory genes. TNFAIP3 was 5.4 fold down-regulated and is an inhibitor of tumour necrosis factor (TNF) induced apoptosis [145]. The 2.6 fold downregulated gene PPP1R13L is capable of inhibiting apoptosis by inhibition of $P_{53}$ [146]. BCL2 is a wellknown suppressor of the apoptotic process and was 2.3 fold down-regulated. BIRC was 2.0 fold down-regulated, and is an inhibitor of apoptosis (IAP) gene, suppressing apoptosis by the inhibition of caspases 3 and 7 [147]. In contrast, there were also down-regulations of apoptosis inducing genes, providing evidence for the inhibition of apoptosis. The TP53BP2 gene has been shown to be able to induce apoptosis through the mitochondrial apoptotic pathway associated with a reduction of the mitochondrial trans-membrane potential and activation of caspase-9 [148] and was 3.0 fold down-regulated. The TNFRSF10B and TNFRSF1B genes, down-regulated 2.4 and 2.1 fold respectively, produce proteins which are members of the TNF-receptor superfamily, and on activation are capable of inducing apoptotic signals. HRK encodes a pro-apoptotic protein which is linked to the mitochondrial apoptotic pathway by possible interaction with the mitochondrial pore-forming protein p32, and was 2.1 fold down-regulated [149]. Another apoptosis inhibitory effect was the 2.4 fold up-regulation of $P R K C Z$, which has been shown to be able to phosphorylate caspase 9 and thereby inhibit the intrinsic apoptotic process [150].

All of the genes from the second cluster were also present in cluster 4 which contained mainly genes involved in transcription, translation and cytoskeleton organisation. The gene expression changes suggested a down-regulation of the transcriptional and translational processes and pointed to changes in cytoskeleton organisation activity. The MAP2 gene was 4.0-fold downregulated and is involved in the organisation and assembly of microtubules [151]. SVIL, which was 3.4-fold down-regulated, encodes a protein which forms a tight link between the actincytoskeleton and the membrane and therefore is thought to have a function in the recruitment of cytoskeleton proteins into the nucleus or the membrane [152]. SPTAN1, also known as alpha fodrin, was 2.1-fold up-regulated and has the ability to bind actin and has therefore been related to the binding of the cytoskeleton to the membrane [153]. Moreover, cleavage of fodrin by the plasmid-encoded toxin (Pet) of enteroaggregative Escherichia coli in epithelial cells results in cytoskeleton disruption [154]. Spectrin beta (SPTBN1) was 2.6-fold up-regulated. This cluster also contained the 2.4-fold down-regulated mitochondrial protein $18 \mathrm{Kda}$ (MTP18) gene. 
Table 3: DAVID functional annotation clustering of the differentially expressed genes from the $70 \mathrm{vso}$ comparison

\begin{tabular}{ll}
\hline Cluster 1 & \\
\hline GO BP Terms & p-value \\
\hline apoptosis & 0.00002 \\
negative regulation of programmed cell death & 0.00148 \\
regulation of apoptosis & 0.01199 \\
regulation of programmed cell death & 0.01287 \\
positive regulation of programmed cell death & 0.28872 \\
\hline Cluster 2 & \\
\hline GO BP Terms & $p$-value \\
\hline negative regulation of protein metabolism & 0.00403 \\
negative regulation of cell organization and biogenesis & 0.04284 \\
regulation of actin polymerization and/or depolymerization & 0.07368 \\
actin polymerization and/or depolymerization & 0.15979 \\
\hline Cluster 3 & \\
\hline GO BP Terms & $p$-value \\
\hline protein kinase cascade & 0.01167 \\
positive regulation of signal transduction & 0.06082 \\
regulation of I-kappaB kinase/NF-kappaB cascade & 0.07747 \\
positive regulation of I-kappaB kinase/NF-kappaB cascade & 0.13082 \\
\hline Cluster 4 & \\
\hline GO BP Terms & $p$-value \\
\hline regulation of protein metabolism & 0.01304 \\
regulation of cellular biosynthesis & 0.01304 \\
regulation of protein biosynthesis & 0.32588 \\
\hline Cluster 5 & 0.63068 \\
\hline GO BP Terms & 0.72054 \\
\hline carboxylic acid transport & 0.00606 \\
organic acid transport & 0.216654 \\
amino acid transport & 0.02320 \\
amine transport & 0.14149 \\
\hline Cluster 6 & 0.22212 \\
\hline GO BP Terms & \\
\hline nitrogen compound biosynthesis & \\
amine biosynthesis & \\
amino acid derivative biosynthesis & \\
\hline biogenic amine metabolism & acid derivative metabolism \\
\hline
\end{tabular}

Six clusters could be identified of which at least one of the gene ontology terms had a p-value $<0.05$. 
Cluster 3 consisted of 23 unique transcripts of which 19 were down- and 4 were up-regulate. The genes in this cluster are mainly related to the processes cell proliferation and differentiation and apoptosis. The fifth cluster contained 9 down-regulated genes suggesting an inhibition of amino acid, lactate, and pyruvate transport. The sixth cluster contained 8 down-regulated transcripts and one up-regulated transcript, of which the gene expression changes pointed towards inhibition of amino acid metabolism.

Table 4: Gene members of the clusters identified by the DAVID functional annotation clustering of the differentially expressed genes from the group 70vso comparison

\begin{tabular}{|c|c|c|c|c|}
\hline \multicolumn{5}{|l|}{ Cluster 1} \\
\hline Genbank Accession No. & Symbol & Title & 2log fold-change & $p$-value \\
\hline NM_005345 & HSPA1A & heat shock 70 kda protein 1a & -4.3 & $3.41 \mathrm{E}-11$ \\
\hline NM_005346 & HSPA1B & heat shock $70 \mathrm{kda}$ protein $1 \mathrm{~b}$ & -4.1 & 1.43E-10 \\
\hline NM_005347 & HSPA5 & heat shock 70kda protein 5 (glucose-regulated protein, $78 \mathrm{kda}$ ) & -3.5 & 7.78E-07 \\
\hline NM_021158 & TRIB3 & tribbles homolog 3 (drosophila) & -3.5 & $1.30 \mathrm{E}-07$ \\
\hline NM_001831 & CLU & clusterin & -3.1 & $8.52 \mathrm{E}-09$ \\
\hline NM_003299 & HSP90B1 & heat shock protein 90kda beta (grp94), member 1 & -2.6 & $1.94 \mathrm{E}-05$ \\
\hline AF022375 & VEGFA & vascular endothelial growth factor & -2.4 & $6.47 \mathrm{E}-10$ \\
\hline NM_006290 & TNFAIP3 & tumour necrosis factor, alpha-induced protein 3 & -2.4 & 3.33E-05 \\
\hline NM_003655 & CBX4 & chromobox homolog 4 (pc class homolog, drosophila) & -2.2 & $1.06 \mathrm{E}-06$ \\
\hline NM_006705 & GADD45G & growth arrest and dna-damage-inducible, gamma & -2.2 & 4.64E-07 \\
\hline L24498 & GADD45A & growth arrest and dna-damage-inducible, alpha & -2.1 & $1.12 \mathrm{E}-05$ \\
\hline NM_015675 & GADD45B & growth arrest and dna-damage-inducible, beta & -2.0 & $5.91 \mathrm{E}-08$ \\
\hline NM_014330 & PPP1R15A & protein phosphatase 1 , regulatory (inhibitor) subunit $15 a$ & -2.0 & 7.15E-08 \\
\hline BC013128 & CEBPG & ccaat/enhancer binding protein (c/ebp), gamma & -1.8 & 3.57E-07 \\
\hline AK022859 & UNC5B & unc-5 homolog b (c. elegans) & -1.7 & 1.49E-04 \\
\hline NM_013246 & CLCF1 & cardiotrophin-like cytokine factor 1 & -1.6 & 2.30E-04 \\
\hline NM_005426 & TP53BP2 & tumour protein $\mathrm{p} 53$ binding protein, 2 & -1.6 & $5.85 \mathrm{E}-08$ \\
\hline AF220656 & BCM:PHLDA1 & pleckstrin homology-like domain, family a, member 1 & -1.6 & 1.24E-04 \\
\hline NM_052815 & IER3 & immediate early response 3 & -1.5 & 7.47E-06 \\
\hline NM_005923 & MAP3K5 & mitogen-activated protein kinase kinase kinase 5 & -1.5 & 4.91E-08 \\
\hline NM_002583 & PAWR & prkc, apoptosis, wt1, regulator & -1.5 & $6.51 \mathrm{E}-05$ \\
\hline NM_033027 & AXUD1 & axin1 up-regulated 1 & -1.4 & $5.04 \mathrm{E}-05$ \\
\hline NM_006663 & PPP1R13L & protein phosphatase 1 , regulatory (inhibitor) subunit 13 like & -1.4 & $1.43 \mathrm{E}-05$ \\
\hline NM_004862 & LITAF & lipopolysaccharide-induced tnf factor & -1.4 & $6.62 \mathrm{E}-04$ \\
\hline NM_004281 & BAG3 & bcl2-associated athanogene 3 & -1.4 & $6.70 \mathrm{E}-05$ \\
\hline AK026181 & BCM:PHLDA1 & pleckstrin homology-like domain, family a, member 1 & -1.3 & 3.05E-04 \\
\hline NM_004343 & CALR & calreticulin & -1.3 & 7.63E-05 \\
\hline AF016266 & TNFRSF10B & tumour necrosis factor receptor superfamily, member 10b & -1.3 & 4.66E-05 \\
\hline AC004832 & SEC14L3 & sec14-like 2 (s. cerevisiae) & -1.3 & $5.48 \mathrm{E}-06$ \\
\hline NM_004052 & BNIP3 & bcl2/adenovirus e1b 19kda interacting protein 3 & -1.2 & 2.22E-04 \\
\hline NM_005627 & SGK & serum/glucocorticoid regulated kinase & -1.2 & $9.99 \mathrm{E}-05$ \\
\hline NM_000633 & BCL2 & b-cell cll/lymphoma 2 & -1.2 & 2.15E-04 \\
\hline & & protein phosphatase 2 (formerly 2a), catalytic subunit, & & \\
\hline NM_002715 & PPP2CA & alpha isoform & -1.1 & 4.37E-04 \\
\hline NM_001066 & TNFRSF1B & tumour necrosis factor receptor superfamily, member $1 \mathrm{~b}$ & -1.1 & $1.12 \mathrm{E}-04$ \\
\hline NM_003806 & HRK & harakiri, bcl2 interacting protein (contains only bh3 domain) & -1.1 & $6.90 \mathrm{E}-05$ \\
\hline NM_003680 & YARS & tyrosyl-trna synthetase & -1.1 & $5.43 \mathrm{E}-06$ \\
\hline NM_002507 & NGFR & nerve growth factor receptor (tnfr superfamily, member 16) & -1.1 & 2.95E-05 \\
\hline NM_006410 & HTATIP2 & hiv-1 tat interactive protein $2,30 \mathrm{kda}$ & -1.1 & $5.29 \mathrm{E}-04$ \\
\hline NM_005087 & FXR1 & fragile $\mathrm{x}$ mental retardation, autosomal homolog 1 & -1.0 & 3.31E-04 \\
\hline AF070674 & $\mathrm{BIRC3}$ & baculoviral iap repeat-containing 3 & -1.0 & 1.15E-04 \\
\hline NM_001904 & CTNNB1 & catenin (cadherin-associated protein), beta $1,88 \mathrm{kda}$ & 1.0 & $3.94 \mathrm{E}-05$ \\
\hline NM_000600 & IL6 & interleukin 6 (interferon, beta 2) & 1.1 & 2.33E-04 \\
\hline
\end{tabular}




\begin{tabular}{|c|c|c|c|c|}
\hline \multirow[t]{2}{*}{ NM_002744 } & \multirow[t]{2}{*}{ PRKCZ } & protein kinase c, zeta & \multirow[t]{2}{*}{1.3} & \multirow[t]{2}{*}{$1.61 \mathrm{E}-04$} \\
\hline & & cyclin-dependent kinase inhibitor 2a & & \\
\hline NM_000077 & CDKN2A & (melanoma, p16, inhibits cdk4) & 1.4 & $4.36 \mathrm{E}-05$ \\
\hline AK021874 & TGFB2 & transforming growth factor, beta 2 & 1.6 & $6.08 \mathrm{E}-07$ \\
\hline NM_002527 & NTF3 & neurotrophin 3 & 2.1 & $4.72 \mathrm{E}-08$ \\
\hline NM_002982 & CCL2 & chemokine (c-c motif) ligand 2 & 2.3 & 4.91E-08 \\
\hline NM_001562 & IL18 & interleukin 18 (interferon-gamma-inducing factor) & 3.2 & 8.13E-08 \\
\hline \multicolumn{5}{|l|}{ Cluster 2} \\
\hline GB Accession No. & Symbol & Title & 2log fold-change & $p$-value \\
\hline NM_031846 & MAP2 & microtubule-associated protein 2 & -2.0 & $1.47 \mathrm{E}-06$ \\
\hline NM_004095 & EIF4EBP1 & eukaryotic translation initiation factor $4 \mathrm{e}$ binding protein 1 & -1.9 & 8.94E-08 \\
\hline NM_000609 & CXCL12 & chemokine (c-x-c motif) ligand 12 (stromal cell-derived factor 1) & -1.8 & $8.75 \mathrm{E}-06$ \\
\hline NM_021738 & SVIL & supervillin & -1.8 & 2.35E-06 \\
\hline \multirow[t]{2}{*}{ NM_022365 } & DNAJC1 & dnaj (hsp40) homolog, subfamily c, member 1 & -1.4 & $1.04 \mathrm{E}-06$ \\
\hline & & protein phosphatase 2 (formerly 2a), catalytic subunit, & & \\
\hline NM_002715 & PPP2CA & alpha isoform & -1.1 & 4.37E-04 \\
\hline NM_003127 & SPTAN1 & spectrin, alpha, non-erythrocytic 1 (alpha-fodrin) & 1.0 & 7.43E-04 \\
\hline NM_000600 & IL6 & interleukin 6 (interferon, beta 2) & 1.1 & 2.33E-04 \\
\hline AK025071 & SPTBN1 & spectrin, beta, non-erythrocytic 1 & 1.1 & $3.72 \mathrm{E}-06$ \\
\hline NM_000177 & GSN & gelsolin (amyloidosis, finnish type) & 1.4 & $6.57 \mathrm{E}-06$ \\
\hline \multicolumn{5}{|l|}{ Cluster 3} \\
\hline GB Accession No. & Symbol & Title & 2log fold-change & $p$-value \\
\hline NM_021158 & TRIB3 & tribbles homolog 3 (drosophila) & -3.5 & $1.30 \mathrm{E}-07$ \\
\hline NM_002133 & HMOX1 & heme oxygenase (decycling) 1 & -3.4 & $5.90 \mathrm{E}-08$ \\
\hline NM_001946 & DUSP6 & dual specificity phosphatase 6 & -2.7 & $3.04 \mathrm{E}-08$ \\
\hline AF022375 & VEGFA & vascular endothelial growth factor & -2.4 & $6.47 \mathrm{E}-10$ \\
\hline NM_006290 & TNFAIP3 & tumour necrosis factor, alpha-induced protein 3 & -2.4 & 3.33E-05 \\
\hline NM_006705 & GADD45G & growth arrest and dna-damage-inducible, gamma & -2.2 & 4.64E-07 \\
\hline \multirow[t]{2}{*}{ NM_015675 } & GADD45B & growth arrest and dna-damage-inducible, beta & -2.0 & $5.91 \mathrm{E}-08$ \\
\hline & & endothelial differentiation, lysophosphatidic acid & & \\
\hline NM_057159 & EDG2 & g-protein-coupled receptor, 2 & -2.0 & $1.65 \mathrm{E}-09$ \\
\hline NM_006622 & PLK2 & polo-like kinase 2 (drosophila) & -1.9 & $1.06 \mathrm{E}-05$ \\
\hline NM_000676 & ADORA2B & adenosine $\mathrm{a} 2 \mathrm{~b}$ receptor & -1.6 & 2.22E-07 \\
\hline NM_013246 & CLCF1 & cardiotrophin-like cytokine factor 1 & -1.6 & $2.30 \mathrm{E}-04$ \\
\hline NM_005923 & MAP3K5 & mitogen-activated protein kinase kinase kinase 5 & -1.5 & 4.91E-08 \\
\hline NM_017572 & MKNK2 & map kinase interacting serine/threonine kinase 2 & -1.4 & 2.34E-04 \\
\hline NM_004862 & LITAF & lipopolysaccharide-induced tnf factor & -1.4 & $6.62 \mathrm{E}-04$ \\
\hline NM_007207 & DUSP10 & dual specificity phosphatase 10 & -1.4 & 2.27E-04 \\
\hline AF016266 & TNFRSF10B & tumour necrosis factor receptor superfamily, member $10 \mathrm{~b}$ & -1.3 & 4.66E-05 \\
\hline \multirow[t]{2}{*}{ NM_004418 } & DUSP2 & dual specificity phosphatase 2 & -1.3 & $1.38 \mathrm{E}-05$ \\
\hline & & protein phosphatase 2 (formerly 2a), catalytic subunit, & & \\
\hline NM_002715 & PPP2CA & alpha isoform & -1.1 & 4.37E-04 \\
\hline NM_000985 & MIT:HsG2227 & ribosomal protein I17 & -1.1 & $1.24 \mathrm{E}-05$ \\
\hline NM_032409 & PINK1 & pten induced putative kinase 1 & 1.1 & $3.42 \mathrm{E}-04$ \\
\hline NM_002982 & CCL2 & chemokine (c-c motif) ligand 2 & 2.3 & 4.91E-08 \\
\hline NM_004335 & BST2 & bone marrow stromal cell antigen 2 & 2.8 & $1.46 \mathrm{E}-06$ \\
\hline NM_000681 & ADRA2A & adrenergic, alpha-2a-, receptor & 3.2 & $2.88 \mathrm{E}-08$ \\
\hline \multicolumn{5}{|l|}{ Cluster 4} \\
\hline GB Accession No. & Symbol & Title & 2log fold-change & $p$-value \\
\hline NM_013246 & CLCF1 & cardiotrophin-like cytokine factor 1 & -1.6 & $2.30 \mathrm{E}-04$ \\
\hline BC013128 & CEBPG & ccaat/enhancer binding protein (c/ebp), gamma & -1.8 & 3.57E-07 \\
\hline NM_000609 & CXCL12 & chemokine (c-x-c motif) ligand 12 (stromal cell-derived factor 1) & -1.8 & $8.75 \mathrm{E}-06$ \\
\hline NM_022365 & DNAJC1 & dnaj (hsp40) homolog, subfamily c, member 1 & -1.4 & $1.04 \mathrm{E}-06$ \\
\hline \multirow[t]{2}{*}{ NM_006260 } & DNAJC3 & dnaj (hsp40) homolog, subfamily c, member 3 & -2.3 & $1.97 \mathrm{E}-07$ \\
\hline & & epidermal growth factor receptor (erythroblastic leukemia & & \\
\hline NM_005228 & EGFR & viral (v-erb-b) oncogene homolog, avian) & -1.4 & 2.44E-06 \\
\hline AL050005 & EIF1 & eukaryotic translation initiation factor 1 & -1.2 & 4.37E-06 \\
\hline NM_005875 & EIF1B & eukaryotic translation initiation factor $1 \mathrm{~b}$ & -1.1 & 4.21E-04 \\
\hline
\end{tabular}




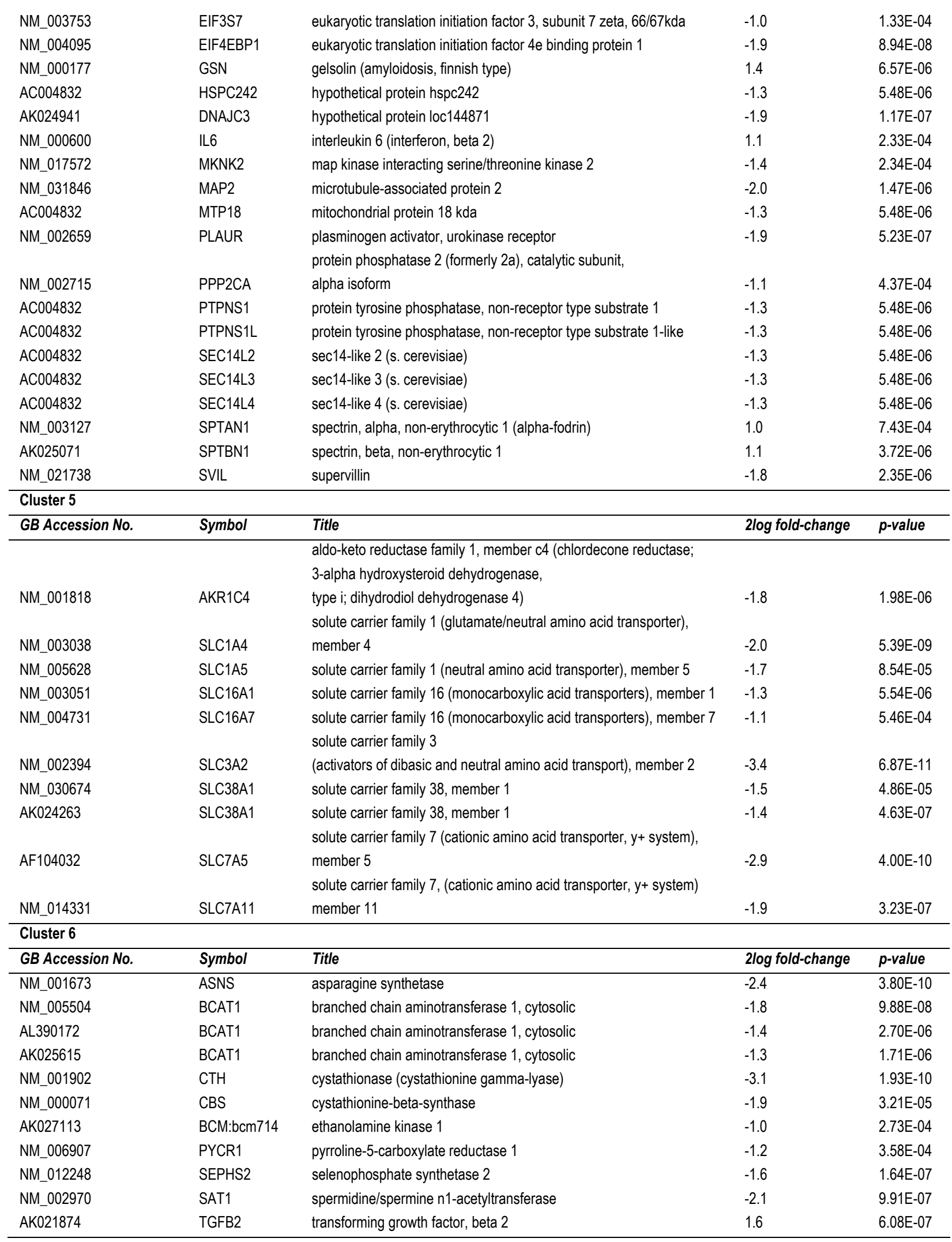


Process based analysis comparison group $97 \%$ versus group 0\%

Functional annotation clustering delivered four gene clusters for the 97vso comparison (tables 5 and 6). For the 97vso comparison, as in the 70vso comparison, the first cluster contained genes with functions related to programmed cell death. Twenty-eight out of the 35 down-regulated genes and four out of the 13 up-regulated genes overlapped with down- and up-regulated genes respectively in the 70vso comparison programmed cell death cluster. Three of the downregulated apoptosis inhibitory genes from the 7ovso comparison were also down-regulated in the 97vso comparison, pointing to stimulation of apoptosis. PPP1R13L, TP53BP2, and HRK were respectively 2.6, 2.3, and 2.3 fold down-regulated. Further apoptosis stimulation was elicited by the 2.6 fold down-regulation of another apoptosis inhibitory gene DEDD2, which is thought to play a role in death-receptor mediated apoptosis by targeting caspases 8 and 10 to the nucleus [155]. In addition, in this comparison also a number of apoptosis stimulatory genes were up-regulated. PDCD4 was 2.3 fold up-regulated, and the PDCD4 protein is believed to be involved in TGF-beta1induced apoptosis [156]. SH3GLB1 has been shown to be able to promote apoptosis by Bax and Bak activation, and was 2.1 fold up-regulated [157]. PYCARD was 2.5 fold up-regulated and promotes apoptosis through activation of caspase 8 [158]. The PDCD6 gene, which was 2.6 fold up-regulated, is involved in apoptosis via the caspase 3 dependent pathway [159, 160]. Inhibition of apoptosis occurred by the 2.4 fold down-regulation of the apoptosis inducing gene LTBR, which promotes apoptosis via TRAF3 and TRAF5 [161-163]. Also, the 2.2 fold up-regulation of the PDCD6IP gene, which is thought to be able to block apoptosis by interacting with PDCD6 [164], points to an inhibition of the apoptotic process. 
Table 5: DAVID functional annotation clustering of the differentially expressed genes from the group 97vso comparison

\begin{tabular}{|c|c|}
\hline Cluster 1 & \\
\hline GO BP Terms & $p$-value \\
\hline apoptosis & 0.00001 \\
\hline negative regulation of programmed cell death & 0.00817 \\
\hline regulation of apoptosis & 0.00922 \\
\hline regulation of programmed cell death & 0.00985 \\
\hline positive regulation of programmed cell death & 0.16247 \\
\hline \multicolumn{2}{|l|}{ Cluster 2} \\
\hline GO BP Terms & p-value \\
\hline regulation of protein metabolism & 0.00085 \\
\hline negative regulation of biosynthesis & 0.00879 \\
\hline regulation of protein biosynthesis & 0.05408 \\
\hline regulation of cellular biosynthesis & 0.06866 \\
\hline \multicolumn{2}{|l|}{ Cluster 3} \\
\hline GO BP Terms & $p$-value \\
\hline Angiogenesis & 0.02557 \\
\hline blood vessel morphogenesis & 0.03139 \\
\hline cell migration & 0.14846 \\
\hline \multicolumn{2}{|l|}{ Cluster 4} \\
\hline GO BP Terms & p-value \\
\hline negative regulation of immune response & 0.04352 \\
\hline regulation of immune response & 0.13106 \\
\hline immune cell activation & 0.32526 \\
\hline lymphocyte activation & 0.35406 \\
\hline lymphocyte differentiation & 0.47940 \\
\hline regulation of lymphocyte activation & 0.53295 \\
\hline positive regulation of immune response & 0.67976 \\
\hline
\end{tabular}

DAVID functional annotation clustering of the differentially expressed genes from the group 97vso comparison. Four clusters could be identified of which at least one of the gene ontology terms had a p-value<0.05.

The second cluster of the 97vso comparison included genes involved in transcriptional and translational processes and cytoskeleton and extracellular matrix (EM) organisation. The gene expression changes pointed to both stimulation and inhibition of these processes and illustrated an increased cytoskeleton and EM organisation activity. Inhibition of cytoskeleton formation is illustrated by the 4.2 fold down-regulation of the MAP2 gene. As already mentioned above, MAP2 is involved in the organisation and assembly of microtubules [151]. The 3.0 fold down-regulation of the TIMP gene results in a protective effect against EM degradation. TIMP1 inhibits matrix metalloproteinases, thereby preventing EM degradation [165]. SPTBN1 was on average 2.7 fold upregulated and is involved in the binding of the cytoskeleton to the membrane (see above). Gelsolin (GSN) is 3.5 fold up-regulated and is able to bind actin filaments as well as to promote polymerisation of barbed ends. GSN is also capable of forming actin filaments by binding two monomeric actin molecules [166]. Furthermore, the up-regulation of GSN is indicative for an apoptosis inhibitory effect, as GSN has been shown to prevent Ras mediated apoptosis [167]. Moreover, GSN appears to provide resistance to apoptosis in senescent fibroblasts [168]. The 
third cluster was labelled with GO terms related to angiogenesis and blood vessel morphogenesis and mainly included genes of which the expression changes pointed towards an inhibition of (endothelial) cell proliferation. The fourth cluster contains genes of which the gene expression changes result in an inhibitory effect on immune response.

Table 6: Gene members of the clusters identified by the DAVID functional annotation clustering of the differentially expressed genes from the $97 v s 0$ comparison

\begin{tabular}{|c|c|c|c|c|}
\hline Genbank Accession No. & Symbol & Title & 2log fold-change & p-value \\
\hline NM_005345 & HSPA1A & heat shock 70kda protein 1a & -4.8 & $1.87 \mathrm{E}-11$ \\
\hline NM_021158 & TRIB3 & tribbles homolog 3 (drosophila) & -4.6 & $1.57 \mathrm{E}-08$ \\
\hline NM_005346 & HSPA1B & $\begin{array}{l}\text { heat shock } 70 \mathrm{kda} \text { protein } 1 \mathrm{a} \\
\text { heat shock } 70 \mathrm{kda} \text { protein } 5\end{array}$ & -4.5 & $1.18 \mathrm{E}-10$ \\
\hline NM_005347 & HSPA5 & (glucose-regulated protein, 78kda) & -3.2 & 7.37E-06 \\
\hline NM_004862 & LITAF & lipopolysaccharide-induced tnf factor & -3.0 & 4.83E-07 \\
\hline L24498 & GADD45A & growth arrest and dna-damage-inducible, alpha & -2.4 & $5.68 \mathrm{E}-06$ \\
\hline NM_015675 & GADD45B & growth arrest and dna-damage-inducible, beta & -2.4 & 2.31E-08 \\
\hline NM_003655 & CBX4 & chromobox homolog 4 ( $\mathrm{pc}$ class homolog, drosophila) & -2.4 & $1.62 \mathrm{E}-06$ \\
\hline NM_006705 & GADD45G & growth arrest and dna-damage-inducible, gamma & -2.3 & $6.91 \mathrm{E}-07$ \\
\hline AF022375 & VEGFA & vascular endothelial growth factor & -2.3 & $9.52 \mathrm{E}-09$ \\
\hline NM_003900 & SQSTM1 & sequestosome 1 & -2.1 & 1.85E-04 \\
\hline NM_002583 & PAWR & prkc, apoptosis, wt1, regulator & -2.1 & $4.88 \mathrm{E}-06$ \\
\hline NM_052815 & IER3 & immediate early response 3 & -2.0 & $5.50 \mathrm{E}-07$ \\
\hline NM_003299 & HSP90B1 & heat shock protein 90kda beta (grp94), member 1 & -2.0 & $7.80 \mathrm{E}-04$ \\
\hline NM_013246 & CLCF1 & cardiotrophin-like cytokine factor 1 & -1.9 & $8.39 \mathrm{E}-05$ \\
\hline NM_014330 & PPP1R15A & protein phosphatase 1 , regulatory (inhibitor) subunit $15 a$ & -1.9 & $4.68 \mathrm{E}-07$ \\
\hline NM_033027 & AXUD1 & axin1 up-regulated 1 & -1.8 & $1.33 \mathrm{E}-05$ \\
\hline NM_021972 & SPHK1 & sphingosine kinase 1 & -1.7 & 4.22E-05 \\
\hline BC013128 & CEBPG & ccaat/enhancer binding protein (c/ebp), gamma & -1.6 & $3.13 \mathrm{E}-06$ \\
\hline NM_004343 & CALR & calreticulin & -1.5 & $4.50 \mathrm{E}-05$ \\
\hline NM_012385 & NUPR1_HUMAN & p8 protein (candidate of metastasis 1 ) & -1.5 & $5.68 \mathrm{E}-06$ \\
\hline NM_000600 & IL6 & interleukin 6 (interferon, beta 2) & -1.5 & 2.91E-05 \\
\hline NM_004281 & BAG3 & bcl2-associated athanogene 3 & -1.5 & $8.02 \mathrm{E}-05$ \\
\hline BC013372 & DEDD2 & death effector domain containing 2 & -1.4 & 1.45E-05 \\
\hline NM_006663 & PPP1R13L & protein phosphatase 1 , regulatory (inhibitor) subunit 13 like & -1.4 & 4.69E-05 \\
\hline NM_002342 & LTBR & lymphotoxin beta receptor (tnfr superfamily, member 3) & -1.3 & $1.39 \mathrm{E}-04$ \\
\hline NM_003680 & YARS & tyrosyl-trna synthetase & -1.3 & $3.13 \mathrm{E}-06$ \\
\hline NM_005426 & TP53BP2 & tumour protein p53 binding protein, 2 & -1.2 & 4.77E-06 \\
\hline NM_003806 & HRK & $\begin{array}{l}\text { harakiri, bcl2 interacting protein (contains only bh3 domain) } \\
\text { transglutaminase } 2 \text { (c polypeptide, protein-glutamine }\end{array}$ & -1.2 & $7.70 \mathrm{E}-05$ \\
\hline NM_004613 & TGM2 & gamma-glutamyltransferase) & -1.2 & $5.67 \mathrm{E}-04$ \\
\hline NM_016639 & TNFRSF12A & tumour necrosis factor receptor superfamily, member 12a & -1.1 & 2.30E-05 \\
\hline NM_006410 & HTATIP2 & hiv-1 tat interactive protein $2,30 \mathrm{kda}$ & -1.1 & $8.71 \mathrm{E}-04$ \\
\hline NM_005627 & SGK & serum/glucocorticoid regulated kinase & -1.1 & $5.98 \mathrm{E}-04$ \\
\hline NM_002507 & NGFR & nerve growth factor receptor (tnfr superfamily, member 16) & -1.1 & $8.02 \mathrm{E}-05$ \\
\hline NM_005923 & MAP3K5 & mitogen-activated protein kinase kinase kinase 5 & -1.0 & $1.73 \mathrm{E}-05$ \\
\hline D25304 & ARHGEF6 & rac/cdc42 guanine nucleotide exchange factor (gef) 6 & 1.0 & $7.88 \mathrm{E}-04$ \\
\hline AB007960 & SH3GLB1 & sh3-domain grb2-like endophilin b1 & 1.1 & $6.82 \mathrm{E}-05$ \\
\hline NM_022173 & TIA1 & tia1 cytotoxic granule-associated rna binding protein & 1.1 & 4.14E-04 \\
\hline AB037796 & PDCD6IP & $\begin{array}{l}\text { programmed cell death } 6 \text { interacting protein } \\
\text { cyclin-dependent kinase inhibitor } 2 a\end{array}$ & 1.2 & $1.34 \mathrm{E}-05$ \\
\hline NM_000077 & CDKN2A & (melanoma, p16, inhibits cdk4) & 1.2 & $9.76 \mathrm{E}-04$ \\
\hline
\end{tabular}




\begin{tabular}{|c|c|c|c|c|}
\hline NM_001904 & CTNNB1 & $\begin{array}{l}\text { catenin (cadherin-associated protein), beta } 1,88 \mathrm{kda} \\
\text { programmed cell death } 4\end{array}$ & 1.2 & $2.54 \mathrm{E}-05$ \\
\hline NM_014456 & PDCD4 & (neoplastic transformation inhibitor) & 1.2 & $9.77 \mathrm{E}-05$ \\
\hline NM_013258 & PYCARD & pyd and card domain containing & 1.3 & $1.64 \mathrm{E}-04$ \\
\hline AB033060 & PDCD6 & programmed cell death 6 & 1.4 & $2.18 \mathrm{E}-06$ \\
\hline AK057865 & THY1 & thy- 1 cell surface antigen & 1.7 & $1.32 \mathrm{E}-04$ \\
\hline NM_000961 & PTGIS & prostaglandin i2 (prostacyclin) synthase & 1.9 & $9.22 \mathrm{E}-04$ \\
\hline NM_002527 & NTF3 & neurotrophin 3 & 2.0 & $3.36 \mathrm{E}-07$ \\
\hline NM_002982 & CCL2 & chemokine (c-c motif) ligand 2 & 3.2 & $1.68 \mathrm{E}-09$ \\
\hline \multicolumn{5}{|l|}{ Cluster 2} \\
\hline Genbank Accession No. & Symbol & Title & 2log fold-change & $p$-value \\
\hline NM_004095 & EIF4EBP1 & eukaryotic translation initiation factor $4 \mathrm{e}$ binding protein 1 & -2.4 & $1.31 \mathrm{E}-08$ \\
\hline NM_006260 & DNAJC3 & dnaj (hsp40) homolog, subfamily c, member 3 & -2.2 & $9.73 \mathrm{E}-07$ \\
\hline NM_000169 & GLA & galactosidase, alpha & -2.1 & $3.47 \mathrm{E}-08$ \\
\hline NM_031846 & MAP2 & microtubule-associated protein 2 & -2.1 & $3.13 \mathrm{E}-06$ \\
\hline NM_013246 & CLCF1 & cardiotrophin-like cytokine factor 1 & -1.9 & $8.39 \mathrm{E}-05$ \\
\hline NM_022365 & DNAJC1 & dnaj (hsp40) homolog, subfamily c, member 1 & -1.8 & $8.28 \mathrm{E}-08$ \\
\hline NM_017572 & MKNK2 & map kinase interacting serine/threonine kinase 2 & -1.7 & $8.00 \mathrm{E}-05$ \\
\hline BC013128 & CEBPG & ccaat/enhancer binding protein (c/ebp), gamma & -1.6 & $3.13 \mathrm{E}-06$ \\
\hline NM_003254 & TIMP1 & timp metallopeptidase inhibitor 1 & -1.6 & $1.80 \mathrm{E}-04$ \\
\hline NM_005875 & EIF1B & eukaryotic translation initiation factor $1 \mathrm{~b}$ & -1.6 & $3.84 \mathrm{E}-05$ \\
\hline NM_003732 & EIF4EBP3 & $\begin{array}{l}\text { eukaryotic translation initiation factor } 4 \text { e binding protein } 3 \\
\text { amyloid beta (a4) precursor protein-binding, }\end{array}$ & -1.6 & $5.66 \mathrm{E}-06$ \\
\hline NM_031232 & APBA2BP & family a, member 2 binding protein & -1.5 & $7.25 \mathrm{E}-07$ \\
\hline NM_000600 & IL6 & interleukin 6 (interferon, beta 2) & -1.5 & $2.91 \mathrm{E}-05$ \\
\hline NM_002659 & PLAUR & plasminogen activator, urokinase receptor & -1.4 & $5.49 \mathrm{E}-05$ \\
\hline AK024941 & DNAJC3 & dnaj (hsp40) homolog, subfamily c, member 3 & -1.4 & $1.51 \mathrm{E}-05$ \\
\hline AL050005 & EIF1 & $\begin{array}{l}\text { eukaryotic translation initiation factor } 1 \\
\text { epidermal growth factor receptor } \\
\text { (erythroblastic leukemia viral (v-erb-b) }\end{array}$ & -1.2 & $8.58 \mathrm{E}-06$ \\
\hline NM_005228 & EGFR & oncogene homolog, avian) & -1.1 & $8.99 \mathrm{E}-05$ \\
\hline AB032972 & ASB1 & ankyrin repeat and socs box-containing 1 & -1.1 & $2.77 \mathrm{E}-04$ \\
\hline AJ420488 & EEF1A1 & eukaryotic translation elongation factor 1 alpha 1 & 1.1 & $8.56 \mathrm{E}-05$ \\
\hline AL117621 & ARF6 & adp-ribosylation factor 6 & 1.1 & $5.06 \mathrm{E}-04$ \\
\hline AK023762 & SPTBN1 & spectrin, beta, non-erythrocytic 1 & 1.4 & $4.21 \mathrm{E}-06$ \\
\hline AK025071 & SPTBN1 & spectrin, beta, non-erythrocytic 1 & 1.5 & $5.98 \mathrm{E}-07$ \\
\hline AK057865 & THY1 & thy- 1 cell surface antigen & 1.7 & $1.32 \mathrm{E}-04$ \\
\hline NM_000177 & GSN & gelsolin (amyloidosis, finnish type) & 1.8 & $7.89 \mathrm{E}-07$ \\
\hline \multicolumn{5}{|l|}{ Cluster 3} \\
\hline Genbank Accession No. & Symbol & Title & 2log fold-change & $p$-value \\
\hline NM_000584 & IL8 & interleukin 8 & -2.4 & $6.61 \mathrm{E}-09$ \\
\hline AL137311 & DNER & delta-notch-like egf repeat-containing transmembrane & -2.3 & $6.64 \mathrm{E}-05$ \\
\hline AF022375 & VEGFA & vascular endothelial growth factor & -2.3 & $9.52 \mathrm{E}-09$ \\
\hline NM_021972 & SPHK1 & $\begin{array}{l}\text { sphingosine kinase } 1 \\
\text { fms-related tyrosine kinase } 1 \\
\text { (vascular endothelial growth factor/ }\end{array}$ & -1.7 & $4.22 \mathrm{E}-05$ \\
\hline NM_002019 & FLT1 & vascular permeability factor receptor) & -1.2 & $2.12 \mathrm{E}-05$ \\
\hline NM_016639 & TNFRSF12A & tumour necrosis factor receptor superfamily, member 12a & -1.1 & $2.30 \mathrm{E}-05$ \\
\hline NM_006410 & HTATIP2 & $\begin{array}{l}\text { hiv- } 1 \text { tat interactive protein } 2,30 \mathrm{kda} \\
\text { epidermal growth factor receptor } \\
\text { (erythroblastic leukemia viral (v-erb-b) }\end{array}$ & -1.1 & $8.71 \mathrm{E}-04$ \\
\hline NM_005228 & EGFR & oncogene homolog, avian) & -1.1 & $8.99 \mathrm{E}-05$ \\
\hline NM_003711 & PPAP2A & phosphatidic acid phosphatase type $2 a$ & 1.3 & $9.67 \mathrm{E}-04$ \\
\hline NM_000859 & HMGCR & 3-hydroxy-3-methylglutaryl-coenzyme a reductase & 1.4 & $3.28 \mathrm{E}-04$ \\
\hline AK057865 & THY1 & thy- 1 cell surface antigen & 1.7 & $1.32 \mathrm{E}-04$ \\
\hline
\end{tabular}




\begin{tabular}{|c|c|c|c|c|}
\hline NM_001150 & ANPEP & $\begin{array}{l}\text { alanyl (membrane) aminopeptidase (aminopeptidase } \mathrm{n} \text {, } \\
\text { aminopeptidase } \mathrm{m} \text {, microsomal aminopeptidase, cd13, p150) }\end{array}$ & 1.8 & $5.96 \mathrm{E}-05$ \\
\hline \multicolumn{5}{|l|}{ Cluster 4} \\
\hline \multirow[t]{2}{*}{ Genbank Accession No. } & Symbol & Title & 2log fold-change & $p$-value \\
\hline & & prostaglandin-endoperoxide synthase 2 & & \\
\hline NM_000963 & PTGS2 & (prostaglandin g/h synthase and cyclooxygenase) & -5.0 & 2.75E-09 \\
\hline NM_000584 & IL8 & interleukin 8 & -2.4 & $6.61 \mathrm{E}-09$ \\
\hline \multirow[t]{2}{*}{ BC013128 } & CEBPG & ccaat/enhancer binding protein (c/ebp), gamma & -1.6 & 3.13E-06 \\
\hline & & dna segment on chromosome $x$ and $y$ (unique) 155 & & \\
\hline NM_005088 & CXYorf3 & expressed sequence, isoform 1 & -1.5 & $8.91 \mathrm{E}-08$ \\
\hline NM_000600 & IL6 & interleukin 6 (interferon, beta 2) & -1.5 & 2.91E-05 \\
\hline NM_018440 & PAG1 & phosphoprotein associated with glycosphingolipid microdomains 1 & -1.2 & $2.58 \mathrm{E}-05$ \\
\hline AB032972 & ASB1 & ankyrin repeat and socs box-containing 1 & -1.1 & 2.77E-04 \\
\hline NM_014011 & socs5 & suppressor of cytokine signaling 5 & 1.5 & 2.27E-07 \\
\hline AK057865 & THY1 & thy-1 cell surface antigen & 1.7 & $1.32 \mathrm{E}-04$ \\
\hline
\end{tabular}

Process based analysis comparison group 97\% versus group 70\%

For the $97 \mathrm{vs70}$ comparison, only one functional gene cluster could be identified for which at least one of gene ontology groups had a p-value $<0.05$ (tables 7 and 8). The cluster was formed by 7 unique transcripts, 4 down- and 3 up-regulated, of which the gene expression changes resulted in an inhibition of the immune response. One of the gene members of this cluster was clusterin (CLU), which was 4.2 fold up-regulated in group $97 \%$ compared to group $70 \%$. Although clusterin has an inhibitory function on the complement system of the innate immune response, it also has been reported to have pro-apoptotic but also pro-survival effects [115].

Table 7: DAVID functional annotation clustering of the differentially expressed genes from the $97 \mathrm{vs70}$ comparison

\begin{tabular}{ll}
\hline Cluster 1 & \\
\hline GO BP Terms & $p$-value \\
\hline humoral immune response & 0.01049 \\
humoral defense mechanism (sensu Vertebrata) & 0.14615 \\
antimicrobial humoral response & 0.26808
\end{tabular}

One cluster could be identified of which at least one of the gene ontology terms had a p-value $<0.05$.

Table 8: Gene members of the cluster identified by the DAVID functional annotation clustering of the differentially expressed genes from the $97 \mathrm{vs70}$ comparison

\begin{tabular}{lllll}
\hline Cluster 1 & & & \\
\hline Genbank Accession No. & Symbol & Title & 2log fold-change & p-value \\
\hline NM_001562 & IL18 & interleukin 18 (interferon-gamma-inducing factor) & -3.0 & $8.12 \mathrm{E}-07$ \\
NM_000600 & IL6 & interleukin 6 (interferon, beta 2) & -2.6 & $5.53 \mathrm{E}-08$ \\
NM_004335 & BST2 & bone marrow stromal cell antigen 2 & -1.7 & $5.44 \mathrm{E}-04$ \\
NM_002413 & MGST2 & microsomal glutathione s-transferase 2 & -1.1 & $7.17 \mathrm{E}-05$ \\
NM_001311 & CRIP1 & cysteine-rich protein 1 (intestinal) & 1.8 & $7.79 \mathrm{E}-05$ \\
NM_001831 & CLU & clusterin & 2.1 & $7.60 \mathrm{E}-06$ \\
NM_030781 & COLEC12 & collectin sub-family member 12 & 2.7 & $5.46 \mathrm{E}-04$ \\
\hline
\end{tabular}


Functional interpretation of the gene expression data

\section{Energy pathways}

The m.9176T>C mutation in the ATP6 gene leads to an OXPHOS defect. Therefore, we checked for gene expression changes in the electron transport chain itself and in the glycolytic pathway, which is able to anaerobically supply energy. For the electron transport chain, only few genes coding for subunits of complexes I and IV, and the ATP synthase complex were significantly changed in expression. In the 70vso comparison, the genes coding for complex I subunit NDUFC2 and complex IV subunit COX7A1 were up-regulated 2.1 and 5.2 fold, respectively. In the 97vso comparison, complex IV subunit COX7A1 gene was 3.5 fold up-regulated and ATP synthase subunit ATP5G2 gene was 2.1 fold down-regulated. The $97 \mathrm{vs70}$ comparison revealed a significant 2.1 fold down-regulation of the ATP1 gene, coding for a subunit of the ATP synthase complex. In the 70vso comparison, the genes enolase 2 (ENO2) and glutamic-oxaloacetic transaminase 1 (GOT1) of the glycolytic pathway were down-regulated 3.4 and 3.7 fold respectively. For the lactate dehydrogenase A ( $L D H A)$ gene, multiple probes were spotted, all indicating a significant increase in expression with an average fold-change of $2.5(\sigma=0.3)$ for the 70vso comparison. In the 97vso comparison, GOT1 was significantly 3.6 fold down-regulated and LDHA showed a significant increase in expression with an average fold-change of $2.5(\sigma=0.2)$. In the $97 \mathrm{vs70}$ comparison, no significant gene expression changes were identified for the glycolytic pathway.

Almost no effort is made by the fibroblast carrying the m.9176T $>C$ mutation to compensate for the OXPHOS defect at the transcriptional level by a direct induction of structural genes of the electron transport chain, as illustrated by the limited gene expression changes in this system. The few gene expression changes in the glycolytic pathway will most likely not have a strong effect on energy metabolism, since the changes do not concern the key regulatory genes of this pathway. Lactic acidosis is one of the biochemical features of Leigh syndrome patients carrying the m.9176T>C mutation [3, 4]. In these patients, pyruvate, which is the end-product of the glycolysis, is accumulated because it is not able to enter the citric acid cycle due to the impaired function of

the ATP synthase complex of the electron transport chain. In order to remove the accumulating pyruvate, it can be converted to lactic acid by lactate dehydrogenase, explaining the upregulation of LDHA in the 70vso and the 97vso comparisons, which leads to the lactic acidosis in these patients. This indicates that the cells are under energy stress.

\section{Programmed cell death and cytoskeleton organisation}

Programmed cell death, or apoptosis, was the main GO biological process which was identified by the DAVID functional clustering analysis in both the 70vso and the 97vso comparisons. For the 70vso comparison, nine apoptosis regulatory genes were identified from the genes from the first cluster of the DAVID functional annotation clustering. Additionally, the down-regulation of MTP18, listed in the fourth functional gene cluster from the DAVID analysis, might increase sensitivity of the cell to apoptotic stimuli. It has been reported that knockdown of the MTP18 protein results in 
cytochrome-c release leading to apoptosis and that a reduced expression of the gene sensitises cells to apoptotic stimuli [169]. Although this might stimulate apoptosis, the majority of the apoptosis stimulatory effects were mostly the result of down-regulated apoptosis inhibitory genes, which in itself is not a direct stimulation of the apoptotic process, whereas the identified down-regulation of apoptosis stimulatory genes is indicative for direct inhibition of the apoptotic process. Therefore, based on these gene expression changes, there appears to be a tendency towards an inhibitory effect of apoptosis in the 7ovso comparison.

For the 97vso comparison, the apoptosis related gene expression changes were also a mixture of inhibitory and stimulatory effects, making it difficult to indicate whether the apoptotic process is stimulated or inhibited or that the data reflects a mixture of cells, which are in different regulatory states with respect to the apoptotic process. The difference with the 7ovso comparison is that in the 97vso comparison more apoptosis stimulatory genes were up-regulated (PDCD4, SH3GLB1, PYCARD, and PDCD6). Another difference between the 70vso and the 97vso comparison is that alpha spectrin and beta spectrin are down-regulated in the 7ovso comparison, whereas alpha spectrin is 2.7 -fold up-regulated in the 97vso comparison. Given the fact that degradation of alphall- and betall-spectrin appears to be an early event in apoptosis [170], there may be a possibility that the spectrins already are being degraded in group $97 \%$ fibroblasts, indicating apoptotic activity, causing the cells to respond with an up-regulation of spectrin alpha. Additionally, it has been suggested that the translocation of actin to mitochondria and the actin cytoskeleton reorganisation, as observed here according to the transcriptional changes of the genes in the second functional cluster of the 97vso comparison (table 6), are early processes in the initiation of apoptosis [171]. Because of these relationships between the cytoskeleton and apoptosis, the other gene expression changes related to cytoskeleton formation and organisation in both the 70vso and the 97vso comparison might be related to the changes in apoptotic gene expression. A trend can be observed from inhibition of apoptosis to stimulation of apoptosis, from the $70 \%$ to the $97 \%$ fibroblasts. This shift to a stimulation of apoptosis is probably also illustrated by the up-regulation of clusterin in the $97 v 570$ comparison. Clusterin has two isoforms, a nuclear form having pro-apoptotic effects and a secreted form having pro-survival effects [115]. Unfortunately, the microarray was unable to distinguish between these two isoforms. Measuring gene expression of the isoforms separately by quantitative realtime PCR will have to provide additional information.

Our results indicate that changes are occurring mainly in the regulation of apoptosis (no gene expression changes of apoptosis key effector genes) in the m.9176T >C fibroblasts and that some early apoptotic events are taking place when the mutation level reaches near homoplasmy. The mixed effects observed in our data may reflect cells in different stages of this process. In a previous paper no stimulation of apoptosis was reported in skeletal muscle of a NARP patient carrying the m.8993 $>C$ mutation at a mutation load of $97 \%$, and only rare apoptotic nuclei were present. This patient had a relatively mild phenotype, compared to the mutation load. Apoptotic 
features, mainly localised in cytochrome c oxidase-negative fibres, were observed in muscle fibres of patients carrying a high percentage of single mtDNA deletions $(>40 \%)$ and of tRNA point mutations (>70\%) [172]. An inhibitory effect on apoptosis by the ATPase6 m.8993T>C and m.9176T>C mutations was proposed in a study where cybrids with and without one of these homoplasmic mutations were transplanted in nude mice. The early stage tumour growth in mice transplanted with these mtATP6 mutations showed larger tumours which grew faster, which could partly be explained by inhibition of apoptosis by the cybrid cells [173]. These findings seem to be inconsistent with a report that fibroblasts harbouring the 8993 MTATP6 mutation were more sensitive to metabolic stress and apoptosis than wild-type fibroblasts [174]. However, this discrepancy is likely to be due to the difference between primary fibroblasts and cancer cells such as HeLa cybrids. For example, the glycolytic pathway in HeLa cells is more active than that in fibroblasts. In a recent study, mitochondrial and ER-stress mediated apoptosis was investigated in relation to a number of different mtDNA mutations, including mitochondrial cybrid cells carrying the homoplasmic NARP m.8993T>G mutation. The authors found that mitochondrial cybrid cells containing the m.8993T $>\mathrm{G}$ mutation were highly susceptible to both mitochondrial and ER-stress mediated apoptosis. It was proposed that the susceptibility of mtDNA mutated cells is related to the presence of OXPHOS complexes and the capability of generating an electron flux [175]. In contrast to the m.9176T $>C$ cells in our study, in the m.8993 $>C$ cells, apoptosis key effects were observed, as illustrated by significantly increased caspase 3 activation. This could be explained by the fact that in the study described, direct apoptosis stimulating agents (staurosporine, etoposide, thapsigargin, or tunicamycin) were used, which is likely to trigger a stronger effect than in a more natural situation as in our cell culturing experiment. Taken all data together, there appears to be no simple and single effect of mtDNA mutations on apoptosis. This depends on mutation type, mutation level and cell or tissue type. However, most data fits with our results where there appears to be a migration from inhibition to stimulation of apoptosis as m.9176T>C mutation loads rise to nearly homoplasmic levels. The controversial nature of the data clearly illustrates the need for a genetically homogeneous model to study the basic effect of these mtDNA mutations.

\section{Conclusion}

The cell culture model system that we have developed is particularly suited to study mtDNA mutation load differences in the same genetic background without the introduction of unwanted experimental and genetic biases. Gene expression profiling of monoclonal fibroblast cell cultures carrying different loads of the m.9176T>C mutation enabled the identification of a number of changes in biological processes between the different mutation load groups, with the most prominent changes in apoptosis with gene expression changes related to cytoskeleton organisation as an apoptotic early event. There appeared to be a shift from an inhibition to a stimulation of apoptosis when m.9176T>C mutation loads rise from $\sim 70 \%$ to $\sim 97 \%$, i.e. from asymptomatic to symptomatic. These biological processes need to be further characterised and 
investigated in more detail, in more tissues and for more patients to provide further insight into the exact role of these processes in the context of this mutation related to Leigh syndrome.

\section{Acknowledgements}

This research was supported by the Princess Beatrix Foundation, (Grant number: MAR99-0111) and the MitoCircle project (EU grant, Sixth Framework Program, contr. no. 005260). 
Supplementary table S1: Significantly differentially expressed genes for the 70 versus 0 comparison

\begin{tabular}{|c|c|c|c|c|}
\hline Genbank accession No. & Gene symbol & Fold-change & Direction & $\mathrm{p}$-value \\
\hline NM_002421 & MMP1 & 55.8 & DOWN & $2.54 \mathrm{E}-10$ \\
\hline NM_002155 & HSPA6 & 50.1 & DOWN & $1.38 \mathrm{E}-11$ \\
\hline NM_000963 & PTGS2 & 31.9 & DOWN & $6.39 \mathrm{E}-10$ \\
\hline NM_001999 & FBN2 & 29.6 & DOWN & $2.02 \mathrm{E}-05$ \\
\hline NM_002923 & RGS2 & 28.2 & DOWN & $2.16 \mathrm{E}-09$ \\
\hline AK056355 & Q7Z3M5_HUMAN & 22.4 & DOWN & $6.33 \mathrm{E}-05$ \\
\hline NM_004165 & RRAD & 22.2 & DOWN & $2.21 \mathrm{E}-11$ \\
\hline NM_002220 & ITPKA & 20.5 & DOWN & $2.92 \mathrm{E}-11$ \\
\hline NM_005345 & HSPA1A & 20.3 & DOWN & $3.41 \mathrm{E}-11$ \\
\hline NM_005345 & HSPA1A & 20.3 & DOWN & $2.21 \mathrm{E}-11$ \\
\hline NM_005345 & HSPA1A & 18.7 & DOWN & $2.66 \mathrm{E}-11$ \\
\hline NM_005345 & HSPA1A & 18.4 & DOWN & $4.88 \mathrm{E}-11$ \\
\hline NM_005345 & HSPA1A & 18.2 & DOWN & $1.38 \mathrm{E}-11$ \\
\hline NM_005345 & HSPA1A & 18.1 & DOWN & $8.11 \mathrm{E}-11$ \\
\hline NM_005345 & HSPA1A & 18.0 & DOWN & $2.86 \mathrm{E}-11$ \\
\hline NM_005345 & HSPA1A & 17.8 & DOWN & $6.87 \mathrm{E}-11$ \\
\hline NM_005532 & IFI27 & 17.6 & DOWN & $1.51 \mathrm{E}-05$ \\
\hline NM_005345 & HSPA1A & 17.4 & DOWN & $1.38 \mathrm{E}-11$ \\
\hline NM_021127 & PMAIP1 & 16.9 & DOWN & 2.85E-09 \\
\hline NM_005345 & HSPA1A & 16.9 & DOWN & $1.06 \mathrm{E}-10$ \\
\hline NM_005346 & HSPA1B & 16.8 & DOWN & $1.43 \mathrm{E}-10$ \\
\hline NM_005345 & HSPA1A & 16.8 & DOWN & $1.58 \mathrm{E}-11$ \\
\hline NM_005345 & HSPA1A & 16.8 & DOWN & $1.38 \mathrm{E}-11$ \\
\hline NM_005345 & HSPA1A & 16.7 & DOWN & $1.38 \mathrm{E}-11$ \\
\hline BC012321 & ARC & 16.6 & DOWN & 3.49E-11 \\
\hline NM_005345 & HSPA1A & 16.5 & DOWN & $1.58 \mathrm{E}-11$ \\
\hline NM_005345 & HSPA1A & 16.4 & DOWN & 1.29E-10 \\
\hline NM_007029 & STMN2 & 16.4 & DOWN & 4.44E-07 \\
\hline NM_005345 & HSPA1A & 16.1 & DOWN & 3.12E-11 \\
\hline NM_005252 & FOS & 15.6 & DOWN & 2.55E-10 \\
\hline NM_004864 & GDF15 & 15.1 & DOWN & $1.46 \mathrm{E}-07$ \\
\hline NM_000799 & EPO & 15.0 & DOWN & 5.17E-09 \\
\hline NM_005345 & HSPA1A & 15.0 & DOWN & $3.41 \mathrm{E}-11$ \\
\hline NM_000361 & THBD & 14.4 & DOWN & $9.43 \mathrm{E}-11$ \\
\hline NM_032621 & BEX2 & 13.9 & DOWN & 2.92E-11 \\
\hline NM_001451 & FOXF1 & 13.6 & DOWN & 1.05E-09 \\
\hline NM_007036 & ESM1 & 12.2 & DOWN & 3.03E-07 \\
\hline NM_006528 & TFPI2 & 12.2 & DOWN & 1.74E-08 \\
\hline NM_001945 & HBEGF & 11.7 & DOWN & 3.04E-08 \\
\hline NM_005347 & HSPA5 & 11.5 & DOWN & 7.78E-07 \\
\hline NM_021158 & TRIB3 & 11.5 & DOWN & $1.30 \mathrm{E}-07$ \\
\hline NM_006145 & DNAJB1 & 11.3 & DOWN & $1.94 \mathrm{E}-11$ \\
\hline NM_002133 & HMOX1 & 10.7 & DOWN & $5.90 \mathrm{E}-08$ \\
\hline NM_004398 & DDX10 & 10.7 & DOWN & $3.40 \mathrm{E}-10$ \\
\hline NM_002394 & SLC3A2 & 10.5 & DOWN & $6.87 \mathrm{E}-11$ \\
\hline NM_005261 & GEM & 10.1 & DOWN & 1.13E-09 \\
\hline NM_001353 & AKR1C1 & 10.0 & DOWN & $3.56 \mathrm{E}-07$ \\
\hline AK001031 & TBX2 & 9.4 & DOWN & $3.94 \mathrm{E}-10$ \\
\hline NM_007115 & TNFAIP6 & 9.4 & DOWN & $1.51 \mathrm{E}-07$ \\
\hline NM_001769 & CD9 & 9.3 & DOWN & 4.81E-11 \\
\hline AB050476 & LHX8 & 9.3 & DOWN & $2.55 \mathrm{E}-10$ \\
\hline NM_006389 & HYOU1 & 9.0 & DOWN & 2.43E-07 \\
\hline NM_004362 & CLGN & 8.9 & DOWN & $5.05 \mathrm{E}-10$ \\
\hline NM_001533 & HNRPL & 8.9 & DOWN & $1.82 \mathrm{E}-10$ \\
\hline NM_017947 & MOCOS & 8.7 & DOWN & $6.64 \mathrm{E}-06$ \\
\hline NM_001902 & CTH & 8.6 & DOWN & 1.93E-10 \\
\hline NM_001831 & CLU & 8.4 & DOWN & $8.52 \mathrm{E}-09$ \\
\hline NM_016084 & RASD1 & 8.3 & DOWN & 3.20E-09 \\
\hline NM_012328 & DNAJB9 & 8.2 & DOWN & $2.73 \mathrm{E}-10$ \\
\hline
\end{tabular}




\begin{tabular}{|c|c|c|c|c|}
\hline BC017001 & SCRT2 & 8.0 & DOWN & $3.30 \mathrm{E}-08$ \\
\hline NM_022842 & CDCP1 & 7.8 & DOWN & $6.57 \mathrm{E}-08$ \\
\hline NM_002852 & PTX3 & 7.6 & DOWN & $8.52 \mathrm{E}-09$ \\
\hline AF104032 & SLC7A5 & 7.5 & DOWN & 4.00E-10 \\
\hline NM_004350 & RUNX3 & 7.3 & DOWN & 1.10E-05 \\
\hline NM_005985 & SNAI1 & 7.0 & DOWN & $6.79 \mathrm{E}-07$ \\
\hline NM_014751 & MTSS1 & 6.9 & DOWN & $6.31 \mathrm{E}-08$ \\
\hline X91348 & C22:AC000095.2 & 6.9 & DOWN & $2.62 \mathrm{E}-04$ \\
\hline NM_022743 & SMYD3 & 6.8 & DOWN & $3.53 \mathrm{E}-08$ \\
\hline AF212221 & MYLIP & 6.7 & DOWN & $1.62 \mathrm{E}-05$ \\
\hline NM_006636 & MTHFD2 & 6.7 & DOWN & 1.67E-10 \\
\hline NM_000627 & LTBP1 & 6.6 & DOWN & $1.36 \mathrm{E}-05$ \\
\hline NM_001946 & DUSP6 & 6.5 & DOWN & $3.04 \mathrm{E}-08$ \\
\hline BC013592 & DDIT4L & 6.4 & DOWN & $9.60 \mathrm{E}-08$ \\
\hline NM_032691 & NA & 6.4 & DOWN & 1.43E-10 \\
\hline NM_002061 & GCLM & 6.3 & DOWN & 7.57E-10 \\
\hline NM_004419 & DUSP5 & 6.3 & DOWN & $1.75 \mathrm{E}-06$ \\
\hline NM_001793 & $\mathrm{CDH} 3$ & 6.3 & DOWN & $4.91 \mathrm{E}-08$ \\
\hline NM_018590 & CGAT2_HUMAN & 6.3 & DOWN & 3.03E-09 \\
\hline AF438313 & TMEM158 & 6.2 & DOWN & 1.46E-07 \\
\hline NM_004024 & ATF3 & 6.1 & DOWN & $5.62 \mathrm{E}-09$ \\
\hline NM_003299 & HSP90B1 & 6.0 & DOWN & $1.94 \mathrm{E}-05$ \\
\hline NM_052966 & NIBA_HUMAN & 5.8 & DOWN & 2.45E-07 \\
\hline NM_004083 & DDIT3 & 5.7 & DOWN & 2.54E-08 \\
\hline NM_016242 & EMCN & 5.6 & DOWN & $1.18 \mathrm{E}-06$ \\
\hline NM_002479 & MYOG & 5.6 & DOWN & 3.79E-07 \\
\hline NM_003012 & SFRP1 & 5.5 & DOWN & 8.83E-04 \\
\hline AF007152 & ABHD3 & 5.5 & DOWN & 2.92E-11 \\
\hline AK056052 & Q7Z3M5_HUMAN & 5.5 & DOWN & 8.23E-05 \\
\hline NM_000167 & GK & 5.5 & DOWN & 3.63E-08 \\
\hline AF022375 & VEGFA & 5.4 & DOWN & $6.47 \mathrm{E}-10$ \\
\hline NM_006732 & FOSB & 5.4 & DOWN & 1.17E-09 \\
\hline NM_006644 & HSPH1 & 5.4 & DOWN & 4.42E-09 \\
\hline NM_006290 & TNFAIP3 & 5.4 & DOWN & 3.33E-05 \\
\hline NM_001673 & ASNS & 5.4 & DOWN & $3.80 \mathrm{E}-10$ \\
\hline NM_006010 & ARMET & 5.4 & DOWN & $5.81 \mathrm{E}-07$ \\
\hline NM_012410 & SEZ6L2 & 5.3 & DOWN & $6.74 \mathrm{E}-09$ \\
\hline NM_000584 & IL8 & 5.3 & DOWN & $1.65 \mathrm{E}-09$ \\
\hline NM_005098 & MSC & 5.3 & DOWN & 2.20E-07 \\
\hline NM_005842 & SPRY2 & 5.2 & DOWN & 1.43E-05 \\
\hline NM_023940 & RASL11B & 5.2 & DOWN & $3.97 \mathrm{E}-08$ \\
\hline NM_019058 & DDIT4 & 5.2 & DOWN & 1.17E-09 \\
\hline NM_020184 & CNNM4 & 5.2 & DOWN & 7.57E-10 \\
\hline AF101051 & CLDN1 & 5.2 & DOWN & $1.41 \mathrm{E}-04$ \\
\hline AF124368 & MIT:C8orf13 & 5.1 & DOWN & $1.77 \mathrm{E}-04$ \\
\hline D31887 & SLC39A14 & 5.1 & DOWN & 4.55E-10 \\
\hline BC016658 & E2F7 & 5.1 & DOWN & 2.72E-07 \\
\hline NM_000231 & SGCG & 5.1 & DOWN & $1.99 \mathrm{E}-06$ \\
\hline NM_025195 & TRIB1 & 4.9 & DOWN & 4.16E-08 \\
\hline NM_057749 & CCNE2 & 4.9 & DOWN & $6.08 \mathrm{E}-07$ \\
\hline NM_006260 & DNAJC3 & 4.9 & DOWN & $1.97 \mathrm{E}-07$ \\
\hline NM_016262 & TUBE1 & 4.9 & DOWN & 2.68E-09 \\
\hline NM_002047 & GARS & 4.8 & DOWN & $5.05 \mathrm{E}-10$ \\
\hline NM_001498 & GCLC & 4.8 & DOWN & 7.57E-10 \\
\hline AB046853 & CDK5RAP2 & 4.8 & DOWN & $7.58 \mathrm{E}-10$ \\
\hline BC016285 & PRKACB & 4.7 & DOWN & 4.35E-09 \\
\hline NM_031456 & FBXW10 & 4.7 & DOWN & $6.04 \mathrm{E}-07$ \\
\hline NM_006931 & SLC2A3 & 4.7 & DOWN & $2.76 \mathrm{E}-10$ \\
\hline NM_003655 & CBX4 & 4.7 & DOWN & 1.06E-06 \\
\hline BC007359 & RTTN & 4.7 & DOWN & 1.97E-08 \\
\hline NM_005402 & RALA & 4.7 & DOWN & $1.02 \mathrm{E}-07$ \\
\hline NM_005279 & GPR1 & 4.6 & DOWN & $1.93 \mathrm{E}-10$ \\
\hline
\end{tabular}




\begin{tabular}{|c|c|c|c|c|}
\hline BC004538 & LONRF1 & 4.6 & DOWN & $4.24 \mathrm{E}-05$ \\
\hline AK001865 & ER01LB & 4.6 & DOWN & $3.41 \mathrm{E}-08$ \\
\hline NM_006705 & GADD45G & 4.5 & DOWN & 4.64E-07 \\
\hline NM_004933 & $\mathrm{CDH} 15$ & 4.5 & DOWN & $1.92 \mathrm{E}-05$ \\
\hline NM_007038 & ADAMTS5 & 4.5 & DOWN & 4.70E-06 \\
\hline AL133096 & DNAJA4 & 4.4 & DOWN & $1.72 \mathrm{E}-06$ \\
\hline NM_013370 & OSGIN1 & 4.4 & DOWN & $3.96 \mathrm{E}-07$ \\
\hline NM_016614 & TTRAP & 4.4 & DOWN & $9.44 \mathrm{E}-08$ \\
\hline M77140 & GAL & 4.4 & DOWN & 1.97E-05 \\
\hline NM_018259 & TTC17 & 4.4 & DOWN & 1.16E-08 \\
\hline AB051479 & VEPH1 & 4.4 & DOWN & $3.51 \mathrm{E}-09$ \\
\hline AB051460 & CPEB4 & 4.4 & DOWN & $9.46 \mathrm{E}-09$ \\
\hline AL050090 & MYRIP & 4.4 & DOWN & 8.77E-07 \\
\hline NM_014191 & SCN8A & 4.3 & DOWN & $3.56 \mathrm{E}-08$ \\
\hline NM_002970 & SAT1 & 4.3 & DOWN & $9.91 \mathrm{E}-07$ \\
\hline NM_006948 & STCH & 4.2 & DOWN & 1.47E-06 \\
\hline NM_004911 & PDIA4 & 4.2 & DOWN & $3.50 \mathrm{E}-05$ \\
\hline NM_000640 & IL13RA2 & 4.2 & DOWN & 8.62E-06 \\
\hline NM_022044 & SDF2L1 & 4.2 & DOWN & 1.17E-07 \\
\hline L24498 & GADD45A & 4.2 & DOWN & $1.12 \mathrm{E}-05$ \\
\hline NM_012323 & MAFF & 4.1 & DOWN & 1.83E-06 \\
\hline NM_006470 & TRIM16 & 4.1 & DOWN & 7.16E-06 \\
\hline NM_015675 & GADD45B & 4.1 & DOWN & $5.91 \mathrm{E}-08$ \\
\hline NM_005080 & XBP1 & 4.1 & DOWN & 2.97E-07 \\
\hline NM_002022 & FMO4 & 4.1 & DOWN & 4.55E-10 \\
\hline NM_014330 & PPP1R15A & 4.0 & DOWN & 7.15E-08 \\
\hline BC015542 & PVR & 4.0 & DOWN & $6.47 \mathrm{E}-05$ \\
\hline NM_057159 & EDG2 & 4.0 & DOWN & 1.65E-09 \\
\hline NM_031846 & MAP2 & 4.0 & DOWN & 1.47E-06 \\
\hline NM_018423 & STYK1 & 4.0 & DOWN & 2.72E-07 \\
\hline NM_001675 & ATF4 & 3.9 & DOWN & $6.57 \mathrm{E}-09$ \\
\hline NM_006096 & NDRG1 & 3.9 & DOWN & $1.10 \mathrm{E}-05$ \\
\hline NM_005596 & NFIB & 3.9 & DOWN & $6.12 \mathrm{E}-06$ \\
\hline AK024261 & NA & 3.9 & DOWN & 2.74E-07 \\
\hline NM_018015 & CXorf57 & 3.9 & DOWN & 2.35E-07 \\
\hline NM_003038 & SLC1A4 & 3.9 & DOWN & 5.39E-09 \\
\hline NM_016522 & NTRI_HUMAN & 3.8 & DOWN & 1.05E-09 \\
\hline NM_002659 & PLAUR & 3.8 & DOWN & 5.23E-07 \\
\hline NM_000876 & IGF2R & 3.8 & DOWN & 2.64E-05 \\
\hline AF212224 & CLK1 & 3.8 & DOWN & 7.44E-07 \\
\hline NM_006622 & PLK2 & 3.8 & DOWN & 1.06E-05 \\
\hline NM_024574 & C4orf31 & 3.8 & DOWN & 4.92E-05 \\
\hline NM_032744 & C6orf105 & 3.8 & DOWN & 1.93E-06 \\
\hline BC010563 & ADAMTS6 & 3.8 & DOWN & 3.47E-06 \\
\hline NM_004095 & EIF4EBP1 & 3.7 & DOWN & $8.94 \mathrm{E}-08$ \\
\hline AF070606 & ATP2B1 & 3.7 & DOWN & $1.72 \mathrm{E}-06$ \\
\hline AK024941 & DNAJC3 & 3.7 & DOWN & 1.17E-07 \\
\hline NM_004733 & SLC33A1 & 3.7 & DOWN & $1.08 \mathrm{E}-09$ \\
\hline NM_001912 & CTSL & 3.7 & DOWN & $3.35 \mathrm{E}-06$ \\
\hline NM_000071 & CBS & 3.7 & DOWN & $3.21 \mathrm{E}-05$ \\
\hline NM_002079 & GOT1 & 3.7 & DOWN & 2.16E-09 \\
\hline NM_014331 & SLC7A11 & 3.6 & DOWN & 3.23E-07 \\
\hline AK022459 & CCPG1 & 3.6 & DOWN & $5.81 \mathrm{E}-07$ \\
\hline NM_013417 & IARS & 3.6 & DOWN & $9.83 \mathrm{E}-07$ \\
\hline AK025379 & PLEKHM1 & 3.6 & DOWN & 4.00E-07 \\
\hline BC012337 & HKDC1 & 3.6 & DOWN & 4.43E-07 \\
\hline NM_017515 & SLC35F2 & 3.6 & DOWN & $3.71 \mathrm{E}-05$ \\
\hline NM_004183 & BEST1 & 3.6 & DOWN & 1.33E-06 \\
\hline AB024574 & GTPBP2 & 3.5 & DOWN & 4.03E-07 \\
\hline AL353933 & SLC22A15 & 3.5 & DOWN & $6.07 \mathrm{E}-06$ \\
\hline NM_005746 & PBEF1 & 3.5 & DOWN & 2.12E-06 \\
\hline NM_005504 & BCAT1 & 3.5 & DOWN & $9.88 \mathrm{E}-08$ \\
\hline
\end{tabular}




\begin{tabular}{|c|c|c|c|c|}
\hline NM_002943 & RORA & 3.5 & DOWN & $1.24 \mathrm{E}-05$ \\
\hline AK055902 & KIAA1324L & 3.5 & DOWN & 4.36E-07 \\
\hline NM_000609 & CXCL12 & 3.5 & DOWN & $8.75 \mathrm{E}-06$ \\
\hline NM_021255 & PELI2 & 3.5 & DOWN & 5.97E-05 \\
\hline NM_004403 & DFNA5 & 3.4 & DOWN & $9.96 \mathrm{E}-08$ \\
\hline AB051541 & KIAA1754 & 3.4 & DOWN & $1.21 \mathrm{E}-05$ \\
\hline NM_007289 & MME & 3.4 & DOWN & 3.69E-04 \\
\hline BC013128 & CEBPG & 3.4 & DOWN & $3.57 \mathrm{E}-07$ \\
\hline NM_004524 & LLGL2 & 3.4 & DOWN & 8.79E-07 \\
\hline NM_006546 & IGF2BP1 & 3.4 & DOWN & 7.23E-06 \\
\hline NM_005502 & ABCA1 & 3.4 & DOWN & $2.13 \mathrm{E}-05$ \\
\hline NM_021738 & SVIL & 3.4 & DOWN & 2.35E-06 \\
\hline NM_001975 & ENO2 & 3.4 & DOWN & $8.98 \mathrm{E}-05$ \\
\hline NM_001818 & AKR1C4 & 3.4 & DOWN & $1.98 \mathrm{E}-06$ \\
\hline NM_005313 & PDIA3 & 3.4 & DOWN & 4.12E-04 \\
\hline NM_004184 & WARS & 3.3 & DOWN & $4.13 \mathrm{E}-07$ \\
\hline AK022859 & UNC5B & 3.3 & DOWN & 1.49E-04 \\
\hline AY044164 & ALPK1 & 3.3 & DOWN & 4.48E-08 \\
\hline NM_023016 & ANKRD57 & 3.3 & DOWN & $5.23 \mathrm{E}-07$ \\
\hline AK055094 & MARVELD2 & 3.3 & DOWN & $1.02 \mathrm{E}-06$ \\
\hline NM_031471 & URP2_HUMAN & 3.3 & DOWN & 1.16E-06 \\
\hline NM_018057 & SLC6A15 & 3.3 & DOWN & 1.04E-04 \\
\hline NM_000025 & ADRB3 & 3.3 & DOWN & $5.90 \mathrm{E}-08$ \\
\hline NM_024111 & CHAC1 & 3.3 & DOWN & $6.46 \mathrm{E}-08$ \\
\hline NM_003582 & DYRK3 & 3.3 & DOWN & 1.07E-04 \\
\hline NM_001695 & ATP6V1C1 & 3.3 & DOWN & 1.17E-07 \\
\hline NM_014059 & C13orf15 & 3.3 & DOWN & 2.98E-04 \\
\hline AJ277587 & SPIRE1 & 3.2 & DOWN & 7.64E-06 \\
\hline NM_032466 & ASPH & 3.2 & DOWN & $3.24 \mathrm{E}-06$ \\
\hline AK056477 & GZF1 & 3.2 & DOWN & $1.50 \mathrm{E}-07$ \\
\hline NM_002014 & FKBP4 & 3.2 & DOWN & $6.62 \mathrm{E}-07$ \\
\hline ВC016556 & TMED5 & 3.2 & DOWN & $3.40 \mathrm{E}-07$ \\
\hline NM_001550 & IFRD1 & 3.2 & DOWN & $3.04 \mathrm{E}-08$ \\
\hline NM_000270 & NP & 3.2 & DOWN & $6.34 \mathrm{E}-07$ \\
\hline NM_000574 & CD55 & 3.2 & DOWN & 3.05E-07 \\
\hline NM_004824 & CDYL & 3.2 & DOWN & 3.88E-07 \\
\hline BC001618 & SLC1A4 & 3.2 & DOWN & 2.28E-09 \\
\hline NM_032315 & SLC25A33 & 3.2 & DOWN & $1.50 \mathrm{E}-05$ \\
\hline NM_000271 & NPC1 & 3.2 & DOWN & $6.95 \mathrm{E}-08$ \\
\hline NM_005628 & SLC1A5 & 3.2 & DOWN & 8.54E-05 \\
\hline NM_032711 & MAFG & 3.2 & DOWN & 7.08E-06 \\
\hline NM_006988 & ADAMTS1 & 3.2 & DOWN & $5.95 \mathrm{E}-06$ \\
\hline D42055 & NEDD4 & 3.2 & DOWN & $9.60 \mathrm{E}-08$ \\
\hline NM_024324 & CRELD2 & 3.2 & DOWN & $5.00 \mathrm{E}-05$ \\
\hline AB033029 & JGI:USP31 & 3.2 & DOWN & 3.07E-07 \\
\hline NM_002359 & MAFG & 3.2 & DOWN & 2.22E-05 \\
\hline NM_005239 & ETS2 & 3.1 & DOWN & $9.29 \mathrm{E}-07$ \\
\hline NM_001400 & EDG1 & 3.1 & DOWN & 2.45E-06 \\
\hline NM_012421 & RLF & 3.1 & DOWN & 1.03E-06 \\
\hline NM_002641 & PIGA & 3.1 & DOWN & $1.73 \mathrm{E}-07$ \\
\hline NM_016569 & TBX3 & 3.1 & DOWN & $1.88 \mathrm{E}-05$ \\
\hline AK025306 & CLK1 & 3.1 & DOWN & $1.30 \mathrm{E}-06$ \\
\hline NM_003364 & UPP1 & 3.1 & DOWN & $9.83 \mathrm{E}-07$ \\
\hline NM_003115 & UAP1 & 3.1 & DOWN & $1.00 \mathrm{E}-05$ \\
\hline AK057846 & NIPA1 & 3.1 & DOWN & $1.84 \mathrm{E}-05$ \\
\hline NM_012248 & SEPHS2 & 3.1 & DOWN & $1.64 \mathrm{E}-07$ \\
\hline NM_003505 & FZD1 & 3.1 & DOWN & $1.37 \mathrm{E}-05$ \\
\hline NM_002467 & MYC & 3.1 & DOWN & $5.83 \mathrm{E}-06$ \\
\hline NM_001681 & ATP2A2 & 3.1 & DOWN & $8.48 \mathrm{E}-06$ \\
\hline AK025100 & SNTB1 & 3.0 & DOWN & 4.48E-05 \\
\hline NM_015208 & ANKRD12 & 3.0 & DOWN & 1.17E-07 \\
\hline AK056446 & HSP90AA1 & 3.0 & DOWN & $1.08 \mathrm{E}-06$ \\
\hline
\end{tabular}




\begin{tabular}{|c|c|c|c|c|}
\hline NM_052960 & RBP7 & 3.0 & DOWN & 7.64E-06 \\
\hline NM_000804 & FOLR3 & 3.0 & DOWN & $5.52 \mathrm{E}-06$ \\
\hline NM_000676 & ADORA2B & 3.0 & DOWN & 2.22E-07 \\
\hline NM_005104 & BRD2_HUMAN & 3.0 & DOWN & $1.91 \mathrm{E}-04$ \\
\hline AL136628 & C4orf16 & 3.0 & DOWN & $3.68 \mathrm{E}-07$ \\
\hline NM_053040 & C1orf79 & 3.0 & DOWN & 1.12E-07 \\
\hline NM_015577 & RAl14 & 3.0 & DOWN & 1.63E-05 \\
\hline NM_016535 & ZNF581 & 3.0 & DOWN & $1.51 \mathrm{E}-06$ \\
\hline NM_005681 & TAF1A & 3.0 & DOWN & $2.67 \mathrm{E}-06$ \\
\hline NM_024642 & GALNT12 & 3.0 & DOWN & 2.25E-06 \\
\hline NM_013246 & CLCF1 & 3.0 & DOWN & 2.30E-04 \\
\hline NM_005426 & TP53BP2 & 3.0 & DOWN & $5.85 \mathrm{E}-08$ \\
\hline NM_016227 & C1orf9 & 3.0 & DOWN & $6.26 \mathrm{E}-06$ \\
\hline AL110126 & NFIB & 3.0 & DOWN & $7.68 \mathrm{E}-05$ \\
\hline NM_025001 & MTHFD2L & 3.0 & DOWN & $3.31 \mathrm{E}-05$ \\
\hline AF220656 & BCM:PHLDA1 & 2.9 & DOWN & $1.24 \mathrm{E}-04$ \\
\hline NM_007076 & NP_009007.2 & 2.9 & DOWN & $1.22 \mathrm{E}-04$ \\
\hline NM_018357 & LARP6 & 2.9 & DOWN & $1.25 \mathrm{E}-06$ \\
\hline NM_024610 & HSPBAP1 & 2.9 & DOWN & 4.33E-06 \\
\hline BC016024 & FOXA3 & 2.9 & DOWN & 2.44E-04 \\
\hline NM_002135 & NR4A1 & 2.9 & DOWN & 4.30E-09 \\
\hline AK056926 & SLC31A1 & 2.9 & DOWN & $3.88 \mathrm{E}-06$ \\
\hline NM_002928 & RGS16 & 2.9 & DOWN & 3.87E-06 \\
\hline AJ301564 & C8orf13 & 2.9 & DOWN & 1.20E-06 \\
\hline NM_003407 & ZFP36 & 2.9 & DOWN & $5.50 \mathrm{E}-04$ \\
\hline NM_005800 & USPL1 & 2.9 & DOWN & 8.14E-07 \\
\hline NM_025084 & Q6P168_HUMAN & 2.9 & DOWN & $7.86 \mathrm{E}-05$ \\
\hline NM_004447 & EPS8 & 2.9 & DOWN & $3.88 \mathrm{E}-06$ \\
\hline BC012513 & RND3 & 2.9 & DOWN & $3.44 \mathrm{E}-06$ \\
\hline NM_018361 & AGPAT5 & 2.9 & DOWN & 2.72E-07 \\
\hline NM_004464 & FGF5 & 2.9 & DOWN & $8.38 \mathrm{E}-04$ \\
\hline NM_001829 & CLCN3 & 2.9 & DOWN & $3.74 \mathrm{E}-06$ \\
\hline AL117608 & FGFR1OP2 & 2.9 & DOWN & $6.30 \mathrm{E}-07$ \\
\hline BC010112 & HSPD1 & 2.9 & DOWN & $5.84 \mathrm{E}-07$ \\
\hline NM_001539 & DNAJA1 & 2.9 & DOWN & 1.17E-07 \\
\hline NM_030751 & SNF1LK & 2.9 & DOWN & $5.32 \mathrm{E}-05$ \\
\hline NM_001122 & ADFP & 2.9 & DOWN & $6.74 \mathrm{E}-05$ \\
\hline AK025800 & NUPL1 & 2.9 & DOWN & $4.19 \mathrm{E}-07$ \\
\hline BC001665 & ABLIM3 & 2.9 & DOWN & 2.46E-05 \\
\hline NM_014778 & NUPL1 & 2.9 & DOWN & 2.18E-05 \\
\hline D26067 & TMEM41B & 2.8 & DOWN & $1.50 \mathrm{E}-07$ \\
\hline NM_003156 & STIM1 & 2.8 & DOWN & 7.68E-04 \\
\hline AK056836 & C22:IL17RA & 2.8 & DOWN & $1.06 \mathrm{E}-06$ \\
\hline BE884686 & LTB4DH & 2.8 & DOWN & $3.23 \mathrm{E}-07$ \\
\hline NM_022751 & FAM59A & 2.8 & DOWN & $1.94 \mathrm{E}-07$ \\
\hline U92285 & GABRE & 2.8 & DOWN & $3.98 \mathrm{E}-06$ \\
\hline NM_033035 & NP_149024.1 & 2.8 & DOWN & 4.88E-04 \\
\hline NM_003244 & TGIF & 2.8 & DOWN & $6.23 \mathrm{E}-06$ \\
\hline NM_003764 & STX11 & 2.8 & DOWN & $9.62 \mathrm{E}-07$ \\
\hline NM_052815 & IER3 & 2.8 & DOWN & 7.47E-06 \\
\hline NM_005527 & HSPA1L & 2.8 & DOWN & $2.12 \mathrm{E}-06$ \\
\hline NM_001516 & GTF2H3 & 2.8 & DOWN & 2.77E-07 \\
\hline NM_014246 & CELSR1 & 2.8 & DOWN & 2.88E-04 \\
\hline BC011763 & TRIM4 & 2.8 & DOWN & 1.25E-06 \\
\hline AK055774 & PAQR3 & 2.8 & DOWN & 1.68E-05 \\
\hline NM_002631 & PGD & 2.8 & DOWN & $6.62 \mathrm{E}-07$ \\
\hline NM_005923 & MAP3K5 & 2.8 & DOWN & $4.91 \mathrm{E}-08$ \\
\hline NM_005110 & GFPT2 & 2.8 & DOWN & $2.74 \mathrm{E}-07$ \\
\hline NM_024692 & RSNL2 & 2.8 & DOWN & 2.27E-07 \\
\hline NM_030674 & SLC38A1 & 2.8 & DOWN & 4.86E-05 \\
\hline NM_002448 & MSX1 & 2.8 & DOWN & $1.88 \mathrm{E}-06$ \\
\hline NM_002714 & PPP1R10 & 2.8 & DOWN & 4.05E-04 \\
\hline
\end{tabular}




\begin{tabular}{|c|c|c|c|c|}
\hline NM_002583 & PAWR & 2.7 & DOWN & $6.51 \mathrm{E}-05$ \\
\hline NM_016306 & DNAJB11 & 2.7 & DOWN & $1.77 \mathrm{E}-05$ \\
\hline AK025661 & LIMS1 & 2.7 & DOWN & 4.35E-07 \\
\hline AL390172 & $\mathrm{NA}$ & 2.7 & DOWN & $2.70 \mathrm{E}-06$ \\
\hline NM_024039 & MIS12 & 2.7 & DOWN & $3.44 \mathrm{E}-09$ \\
\hline NM_000637 & GSR & 2.7 & DOWN & $3.10 \mathrm{E}-05$ \\
\hline NM_014161 & MRPL18 & 2.7 & DOWN & 1.17E-07 \\
\hline NM_016947 & G8_HUMAN & 2.7 & DOWN & $3.56 \mathrm{E}-07$ \\
\hline NM_000956 & PTGER2 & 2.7 & DOWN & $8.29 \mathrm{E}-05$ \\
\hline AB023142 & CORO2B & 2.7 & DOWN & 2.67E-05 \\
\hline NM_020645 & NRIP3 & 2.7 & DOWN & $3.32 \mathrm{E}-06$ \\
\hline NM_002090 & CXCL3 & 2.7 & DOWN & $1.02 \mathrm{E}-05$ \\
\hline AK055618 & C18orf19 & 2.7 & DOWN & $5.19 \mathrm{E}-06$ \\
\hline NM_033027 & AXUD1 & 2.7 & DOWN & $5.04 \mathrm{E}-05$ \\
\hline AL049265 & IL6ST & 2.7 & DOWN & $1.58 \mathrm{E}-05$ \\
\hline AB046769 & Q9H0M3_HUMAN & 2.6 & DOWN & $2.75 \mathrm{E}-06$ \\
\hline NM_002182 & IL1RAP & 2.6 & DOWN & $1.39 \mathrm{E}-05$ \\
\hline AK056433 & VAC14 & 2.6 & DOWN & $1.99 \mathrm{E}-05$ \\
\hline NM_006043 & HS3ST2 & 2.6 & DOWN & $2.62 \mathrm{E}-05$ \\
\hline NM_003183 & ADAM17 & 2.6 & DOWN & $3.68 \mathrm{E}-07$ \\
\hline NM_017572 & MKNK2 & 2.6 & DOWN & $2.34 \mathrm{E}-04$ \\
\hline AK024263 & NA & 2.6 & DOWN & 4.63E-07 \\
\hline NM_006663 & PPP1R13L & 2.6 & DOWN & $1.43 \mathrm{E}-05$ \\
\hline NM_032883 & GCX1_HUMAN & 2.6 & DOWN & $1.48 \mathrm{E}-04$ \\
\hline NM_002835 & PTPN12 & 2.6 & DOWN & 4.30E-07 \\
\hline NM_004862 & LITAF & 2.6 & DOWN & $6.62 \mathrm{E}-04$ \\
\hline NM_005729 & PPIF & 2.6 & DOWN & $1.21 \mathrm{E}-06$ \\
\hline NM_007207 & DUSP10 & 2.6 & DOWN & 2.27E-04 \\
\hline AY032950 & TRPM7 & 2.6 & DOWN & $1.31 \mathrm{E}-06$ \\
\hline NM_005228 & EGFR & 2.6 & DOWN & 2.44E-06 \\
\hline BC017253 & U31 & 2.6 & DOWN & $5.02 \mathrm{E}-05$ \\
\hline BC010990 & ZNF598 & 2.6 & DOWN & $8.74 \mathrm{E}-06$ \\
\hline BC014523 & Q7Z6M3_HUMAN & 2.6 & DOWN & $5.00 \mathrm{E}-05$ \\
\hline NM_003580 & NSMAF & 2.6 & DOWN & $1.39 \mathrm{E}-05$ \\
\hline NM_004987 & LIMS1 & 2.6 & DOWN & $6.47 \mathrm{E}-05$ \\
\hline NM_014584 & ER01L & 2.6 & DOWN & $1.33 \mathrm{E}-06$ \\
\hline AB011164 & NP_001005751.1 & 2.6 & DOWN & $1.45 \mathrm{E}-08$ \\
\hline NM_001270 & $\mathrm{CHD} 1$ & 2.6 & DOWN & 1.95E-06 \\
\hline NM_014167 & CCDC59 & 2.6 & DOWN & $4.03 \mathrm{E}-06$ \\
\hline NM_005088 & CXYorf3 & 2.6 & DOWN & $9.96 \mathrm{E}-08$ \\
\hline AB058745 & KBTBD8 & 2.6 & DOWN & $6.71 \mathrm{E}-04$ \\
\hline NM_000882 & IL12A & 2.6 & DOWN & $2.20 \mathrm{E}-05$ \\
\hline NM_018112 & TMEM38B & 2.6 & DOWN & 8.77E-04 \\
\hline NM_000201 & ICAM1 & 2.6 & DOWN & $6.17 \mathrm{E}-05$ \\
\hline AB033112 & BRPF3 & 2.6 & DOWN & $3.04 \mathrm{E}-07$ \\
\hline NM_012124 & CHORDC1 & 2.6 & DOWN & $8.94 \mathrm{E}-08$ \\
\hline NM_002064 & GLRX & 2.6 & DOWN & $2.11 \mathrm{E}-04$ \\
\hline NM_022365 & DNAJC1 & 2.6 & DOWN & $1.04 \mathrm{E}-06$ \\
\hline NM_004281 & BAG3 & 2.6 & DOWN & $6.70 \mathrm{E}-05$ \\
\hline NM_004787 & SLIT2 & 2.5 & DOWN & 2.23E-04 \\
\hline AK024224 & ER01L & 2.5 & DOWN & 4.21E-05 \\
\hline NM_017909 & C6orf96 & 2.5 & DOWN & $7.06 \mathrm{E}-06$ \\
\hline NM_004272 & HOMER1 & 2.5 & DOWN & $5.45 \mathrm{E}-06$ \\
\hline NM_005493 & RANBP9 & 2.5 & DOWN & $3.06 \mathrm{E}-05$ \\
\hline AK026095 & SNTB1 & 2.5 & DOWN & $9.87 \mathrm{E}-05$ \\
\hline AK026181 & BCM:PHLDA1 & 2.5 & DOWN & 3.05E-04 \\
\hline NM_024506 & GLB1L & 2.5 & DOWN & $1.00 \mathrm{E}-04$ \\
\hline NM_004414 & DSCR1 & 2.5 & DOWN & 7.94E-06 \\
\hline NM_005544 & IRS1 & 2.5 & DOWN & $1.04 \mathrm{E}-05$ \\
\hline NM_001859 & SLC31A1 & 2.5 & DOWN & $3.24 \mathrm{E}-06$ \\
\hline NM_024525 & TTC13 & 2.5 & DOWN & $1.43 \mathrm{E}-05$ \\
\hline AK055903 & KITLG & 2.5 & DOWN & $4.45 \mathrm{E}-05$ \\
\hline
\end{tabular}




\begin{tabular}{|c|c|c|c|c|}
\hline AK025615 & NA & 2.5 & DOWN & $1.71 \mathrm{E}-06$ \\
\hline NM_001457 & FLNB & 2.5 & DOWN & 3.47E-05 \\
\hline NM_005524 & HES1 & 2.5 & DOWN & 1.46E-06 \\
\hline NM_006516 & SLC2A1 & 2.5 & DOWN & 4.12E-06 \\
\hline NM_006072 & CCL26 & 2.5 & DOWN & 3.17E-05 \\
\hline NM_003051 & SLC16A1 & 2.5 & DOWN & $5.54 \mathrm{E}-06$ \\
\hline AF444779 & SYNE1 & 2.5 & DOWN & 8.93E-06 \\
\hline NM_004990 & MARS & 2.5 & DOWN & 2.89E-05 \\
\hline AF055007 & MARCH3 & 2.5 & DOWN & $7.98 \mathrm{E}-05$ \\
\hline NM_022840 & METTL4 & 2.5 & DOWN & $1.21 \mathrm{E}-05$ \\
\hline NM_006769 & LMO4 & 2.5 & DOWN & 1.70E-06 \\
\hline NM_005354 & JUND & 2.5 & DOWN & 5.49E-04 \\
\hline NM_032839 & DIRC2 & 2.5 & DOWN & $3.96 \mathrm{E}-05$ \\
\hline NM_004343 & CALR & 2.5 & DOWN & 7.63E-05 \\
\hline NM_001134 & AFP & 2.5 & DOWN & $8.50 \mathrm{E}-06$ \\
\hline NM_001568 & EIF3S6 & 2.5 & DOWN & 4.27E-04 \\
\hline NM_022447 & PAPD5 & 2.5 & DOWN & $1.02 \mathrm{E}-04$ \\
\hline AB018274 & LARP1 & 2.5 & DOWN & $4.21 \mathrm{E}-04$ \\
\hline NM_001881 & CREM & 2.5 & DOWN & $2.08 \mathrm{E}-06$ \\
\hline NM_006681 & NMU & 2.5 & DOWN & $3.71 \mathrm{E}-05$ \\
\hline NM_002106 & $\mathrm{H} 2 \mathrm{AFZ}$ & 2.5 & DOWN & $6.23 \mathrm{E}-05$ \\
\hline NM_012463 & ATP6V0A2 & 2.5 & DOWN & $3.42 \mathrm{E}-05$ \\
\hline NM_002526 & NT5E & 2.4 & DOWN & $3.40 \mathrm{E}-05$ \\
\hline NM_001895 & CSNK2A1 & 2.4 & DOWN & $6.65 \mathrm{E}-05$ \\
\hline NM_012296 & GAB2 & 2.4 & DOWN & 1.87E-04 \\
\hline NM_003340 & UBE2D3 & 2.4 & DOWN & $3.90 \mathrm{E}-05$ \\
\hline NM_014674 & EDEM1 & 2.4 & DOWN & $5.85 \mathrm{E}-07$ \\
\hline NM_005655 & KLF10 & 2.4 & DOWN & 7.03E-04 \\
\hline AL021327 & RP1-12409.1 & 2.4 & DOWN & 2.63E-07 \\
\hline BC008580 & GDNF & 2.4 & DOWN & $3.04 \mathrm{E}-06$ \\
\hline NM_004673 & ANGPTL1 & 2.4 & DOWN & $1.63 \mathrm{E}-05$ \\
\hline NM_020038 & $\mathrm{ABCC} 3$ & 2.4 & DOWN & 7.65E-04 \\
\hline AF016266 & TNFRSF10B & 2.4 & DOWN & 4.66E-05 \\
\hline AC004832 & SEC14L3 & 2.4 & DOWN & $5.48 \mathrm{E}-06$ \\
\hline D50926 & MORC3 & 2.4 & DOWN & $1.38 \mathrm{E}-07$ \\
\hline NM_001751 & CARS & 2.4 & DOWN & 1.19E-05 \\
\hline NM_004418 & DUSP2 & 2.4 & DOWN & $1.38 \mathrm{E}-05$ \\
\hline BC013764 & KCTD12 & 2.4 & DOWN & $1.13 \mathrm{E}-05$ \\
\hline NM_032270 & LRRC8C & 2.4 & DOWN & $1.39 \mathrm{E}-05$ \\
\hline NM_002041 & GABPAP & 2.4 & DOWN & $1.34 \mathrm{E}-06$ \\
\hline NM_006670 & TPBG & 2.4 & DOWN & $2.26 \mathrm{E}-04$ \\
\hline NM_003171 & SUPV3L1 & 2.4 & DOWN & 7.64E-06 \\
\hline NM_003368 & USP1 & 2.4 & DOWN & $3.42 \mathrm{E}-06$ \\
\hline AB018285 & JMJD1A & 2.4 & DOWN & $9.50 \mathrm{E}-07$ \\
\hline NM_003155 & STC1 & 2.4 & DOWN & $1.10 \mathrm{E}-05$ \\
\hline NM_032873 & STS1_HUMAN & 2.4 & DOWN & 7.36E-05 \\
\hline NM_003033 & ST3GAL1 & 2.4 & DOWN & $1.04 \mathrm{E}-04$ \\
\hline NM_001746 & CANX & 2.4 & DOWN & $8.05 \mathrm{E}-07$ \\
\hline NM_017793 & RPP25 & 2.4 & DOWN & 7.34E-05 \\
\hline NM_006819 & STIP1 & 2.4 & DOWN & $3.69 \mathrm{E}-04$ \\
\hline D83702 & CRY1 & 2.4 & DOWN & $2.67 \mathrm{E}-06$ \\
\hline NM_003489 & NRIP1 & 2.4 & DOWN & $6.39 \mathrm{E}-07$ \\
\hline NM_014423 & AFF4 & 2.4 & DOWN & $6.11 \mathrm{E}-06$ \\
\hline AB011542 & MEGF9 & 2.4 & DOWN & $4.96 \mathrm{E}-04$ \\
\hline NM_024755 & SLTM & 2.4 & DOWN & $1.01 \mathrm{E}-05$ \\
\hline NM_020424 & LYRM1 & 2.4 & DOWN & $5.95 \mathrm{E}-06$ \\
\hline AF278605 & SEC31A & 2.4 & DOWN & $5.88 \mathrm{E}-07$ \\
\hline NM_005896 & IDH1 & 2.4 & DOWN & $2.81 \mathrm{E}-04$ \\
\hline ВC009033 & ASPHD1 & 2.3 & DOWN & $5.25 \mathrm{E}-05$ \\
\hline NM_003851 & CREG1 & 2.3 & DOWN & $2.06 \mathrm{E}-04$ \\
\hline NM_003670 & BHLHB2 & 2.3 & DOWN & $4.70 \mathrm{E}-06$ \\
\hline NM_000558 & HBA_HUMAN & 2.3 & DOWN & $2.34 \mathrm{E}-05$ \\
\hline
\end{tabular}




\begin{tabular}{|c|c|c|c|c|}
\hline NM_006362 & NXF1 & 2.3 & DOWN & $4.48 \mathrm{E}-06$ \\
\hline NM_014710 & GPRASP1 & 2.3 & DOWN & $7.22 \mathrm{E}-05$ \\
\hline NM_031412 & GABARAPL1 & 2.3 & DOWN & $6.36 \mathrm{E}-04$ \\
\hline NM_002423 & MMP7 & 2.3 & DOWN & $6.47 \mathrm{E}-05$ \\
\hline NM_024686 & TTLL7 & 2.3 & DOWN & $1.46 \mathrm{E}-06$ \\
\hline NM_023076 & C16orf28 & 2.3 & DOWN & $1.81 \mathrm{E}-05$ \\
\hline AB037801 & JMJD1C & 2.3 & DOWN & $1.97 \mathrm{E}-05$ \\
\hline AB058713 & SYVN1 & 2.3 & DOWN & 5.65E-04 \\
\hline AK026142 & GATAD1 & 2.3 & DOWN & 8.83E-04 \\
\hline BC009367 & ZNF317 & 2.3 & DOWN & $1.19 \mathrm{E}-04$ \\
\hline NM_004052 & BNIP3 & 2.3 & DOWN & 2.22E-04 \\
\hline NM_014278 & HSPA4L & 2.3 & DOWN & 1.11E-04 \\
\hline NM_000942 & PPIB & 2.3 & DOWN & $4.98 \mathrm{E}-04$ \\
\hline NM_022750 & PARP12 & 2.3 & DOWN & $2.72 \mathrm{E}-04$ \\
\hline AK027647 & DNAJC10 & 2.3 & DOWN & $6.20 \mathrm{E}-06$ \\
\hline AK026747 & Q9UMH3_HUMAN & 2.3 & DOWN & $6.00 \mathrm{E}-04$ \\
\hline NM_000169 & GLA & 2.3 & DOWN & $1.00 \mathrm{E}-05$ \\
\hline NM_006492 & ALX3 & 2.3 & DOWN & $4.13 \mathrm{E}-04$ \\
\hline AK021570 & NA & 2.3 & DOWN & 3.95E-05 \\
\hline NM_005727 & TSPAN1 & 2.3 & DOWN & 4.37E-06 \\
\hline NM_002019 & FLT1 & 2.3 & DOWN & $7.51 \mathrm{E}-06$ \\
\hline NM_006537 & USP3 & 2.3 & DOWN & 2.12E-06 \\
\hline AB051505 & SK:KIAA1718 & 2.3 & DOWN & $5.59 \mathrm{E}-05$ \\
\hline NM_005627 & SGK & 2.3 & DOWN & $9.99 \mathrm{E}-05$ \\
\hline NM_004087 & DLG1 & 2.3 & DOWN & 1.99E-04 \\
\hline NM_000158 & GBE1 & 2.3 & DOWN & $1.30 \mathrm{E}-06$ \\
\hline NM_003243 & TGFBR3 & 2.3 & DOWN & $1.21 \mathrm{E}-06$ \\
\hline NM_000633 & BCL2 & 2.3 & DOWN & 2.15E-04 \\
\hline NM_024303 & ZSCAN5 & 2.3 & DOWN & 3.23E-04 \\
\hline AK021789 & Q8NHV5_HUMAN & 2.3 & DOWN & 4.04E-05 \\
\hline NM_031431 & $\operatorname{COG} 3$ & 2.3 & DOWN & $4.51 \mathrm{E}-06$ \\
\hline AK055243 & CCBE1 & 2.3 & DOWN & 1.87E-04 \\
\hline AK055975 & ZNF746 & 2.3 & DOWN & $3.10 \mathrm{E}-04$ \\
\hline AB040916 & ZBTB2 & 2.3 & DOWN & $5.34 \mathrm{E}-05$ \\
\hline BC010612 & Q96FP1_HUMAN & 2.3 & DOWN & $3.59 \mathrm{E}-05$ \\
\hline NM_014914 & CENTG2 & 2.3 & DOWN & 5.83E-06 \\
\hline NM_012290 & TLK1 & 2.3 & DOWN & $1.32 \mathrm{E}-05$ \\
\hline AF395440 & Q96RF1_HUMAN & 2.3 & DOWN & $3.92 \mathrm{E}-05$ \\
\hline NM_016325 & ZNF274 & 2.3 & DOWN & $6.26 \mathrm{E}-07$ \\
\hline NM_022780 & RMND5A & 2.3 & DOWN & 2.75E-06 \\
\hline BC012044 & SGTB & 2.3 & DOWN & 1.07E-04 \\
\hline NM_000179 & MSH6 & 2.3 & DOWN & $1.71 \mathrm{E}-04$ \\
\hline AK023505 & RSNL2 & 2.3 & DOWN & 8.89E-04 \\
\hline NM_006392 & NOL5A & 2.3 & DOWN & 7.64E-06 \\
\hline NM_003662 & PIR & 2.2 & DOWN & $9.02 \mathrm{E}-04$ \\
\hline NM_003576 & STK24 & 2.2 & DOWN & 1.47E-05 \\
\hline NM_006907 & PYCR1 & 2.2 & DOWN & $3.58 \mathrm{E}-04$ \\
\hline NM_006693 & CPSF4 & 2.2 & DOWN & 1.76E-05 \\
\hline NM_003344 & UBE2H & 2.2 & DOWN & 7.82E-05 \\
\hline NM_015513 & CRELD1 & 2.2 & DOWN & 1.25E-04 \\
\hline NM_030759 & NRBF2 & 2.2 & DOWN & $1.98 \mathrm{E}-06$ \\
\hline NM_006756 & TCEA1_HUMAN & 2.2 & DOWN & $7.62 \mathrm{E}-06$ \\
\hline AB051498 & ZCCHC6 & 2.2 & DOWN & $5.54 \mathrm{E}-06$ \\
\hline AK057782 & ANKRD29 & 2.2 & DOWN & $9.10 \mathrm{E}-04$ \\
\hline NM_016545 & IER5 & 2.2 & DOWN & $1.86 \mathrm{E}-05$ \\
\hline NM_020122 & KCMF1 & 2.2 & DOWN & $1.02 \mathrm{E}-06$ \\
\hline AB051513 & ZC3H12C & 2.2 & DOWN & $9.84 \mathrm{E}-06$ \\
\hline NM_002243 & KCNJ15 & 2.2 & DOWN & $8.41 \mathrm{E}-04$ \\
\hline NM_003567 & BCAR3 & 2.2 & DOWN & 4.23E-04 \\
\hline NM_021958 & HLX1 & 2.2 & DOWN & $5.11 \mathrm{E}-04$ \\
\hline NM_000735 & CGA & 2.2 & DOWN & 7.86E-05 \\
\hline AL050005 & EIF1 & 2.2 & DOWN & 4.37E-06 \\
\hline
\end{tabular}




\begin{tabular}{|c|c|c|c|c|}
\hline NM_000079 & CHRNA1 & 2.2 & DOWN & $1.08 \mathrm{E}-05$ \\
\hline NM_002252 & KCNS3 & 2.2 & DOWN & 3.05E-04 \\
\hline NM_002056 & GFPT1 & 2.2 & DOWN & 7.18E-05 \\
\hline NM_002078 & GOLGA4 & 2.2 & DOWN & 3.17E-04 \\
\hline AK055649 & ERICH1 & 2.2 & DOWN & 4.51E-06 \\
\hline NM_020233 & C17orf48 & 2.2 & DOWN & 7.82E-05 \\
\hline AB051516 & NP_444270.2 & 2.2 & DOWN & $3.41 \mathrm{E}-06$ \\
\hline AK000933 & CHML & 2.2 & DOWN & $9.62 \mathrm{E}-07$ \\
\hline NM_031459 & SESN2 & 2.2 & DOWN & $2.88 \mathrm{E}-04$ \\
\hline AK027789 & STT3B & 2.2 & DOWN & $3.02 \mathrm{E}-05$ \\
\hline NM_000311 & PRNP & 2.2 & DOWN & 7.82E-05 \\
\hline AB059277 & AHCTF1 & 2.2 & DOWN & 2.96E-05 \\
\hline AL110139 & NP_997329.1 & 2.2 & DOWN & $3.95 \mathrm{E}-04$ \\
\hline BC017117 & CREM & 2.2 & DOWN & 1.27E-06 \\
\hline NM_006746 & SCML1 & 2.2 & DOWN & 7.17E-05 \\
\hline NM_007314 & $\mathrm{ABL} 2$ & 2.2 & DOWN & 5.61E-06 \\
\hline NM_000710 & BDKRB1 & 2.2 & DOWN & 3.27E-05 \\
\hline NM_005875 & EIF1B & 2.2 & DOWN & 4.21E-04 \\
\hline NM_052868 & IGSF8 & 2.2 & DOWN & $1.28 \mathrm{E}-04$ \\
\hline NM_002219 & STT3A & 2.2 & DOWN & $1.01 \mathrm{E}-04$ \\
\hline AB058747 & WAC & 2.2 & DOWN & 4.13E-04 \\
\hline NM_004731 & SLC16A7 & 2.2 & DOWN & $5.46 \mathrm{E}-04$ \\
\hline NM_000081 & LYST & 2.2 & DOWN & 3.17E-05 \\
\hline NM_032895 & NP_001001870.1 & 2.2 & DOWN & $4.82 \mathrm{E}-05$ \\
\hline NM_002715 & PPP2CA & 2.2 & DOWN & 4.37E-04 \\
\hline NM_006207 & PDGFRL & 2.2 & DOWN & $1.75 \mathrm{E}-04$ \\
\hline AF148949 & NA & 2.2 & DOWN & $6.51 \mathrm{E}-04$ \\
\hline NM_000935 & PLOD2 & 2.2 & DOWN & 7.71E-05 \\
\hline NM_024633 & CN139_HUMAN & 2.2 & DOWN & 4.35E-07 \\
\hline AL136807 & NP_055260.1 & 2.2 & DOWN & $2.38 \mathrm{E}-04$ \\
\hline BC011715 & Q8WTY6_HUMAN & 2.2 & DOWN & 2.32E-04 \\
\hline NM_021194 & SLC30A1 & 2.2 & DOWN & 4.19E-04 \\
\hline AK024896 & SLC5A3 & 2.2 & DOWN & 2.86E-06 \\
\hline AK026902 & GNA13 & 2.2 & DOWN & 8.64E-06 \\
\hline AK022547 & PARP6 & 2.2 & DOWN & 1.77E-05 \\
\hline NM_005587 & MEF2A & 2.2 & DOWN & 1.97E-05 \\
\hline NM_002717 & DOCK5 & 2.1 & DOWN & $1.22 \mathrm{E}-05$ \\
\hline NM_001066 & TNFRSF1B & 2.1 & DOWN & $1.12 \mathrm{E}-04$ \\
\hline AK057820 & PTGES3 & 2.1 & DOWN & $6.44 \mathrm{E}-06$ \\
\hline AJ420423 & UGCG & 2.1 & DOWN & $6.70 \mathrm{E}-05$ \\
\hline NM_002846 & PTPRN & 2.1 & DOWN & 4.24E-04 \\
\hline AB007931 & ZUBR1 & 2.1 & DOWN & 1.77E-05 \\
\hline NM_003806 & HRK & 2.1 & DOWN & $6.90 \mathrm{E}-05$ \\
\hline AK023623 & GLDN & 2.1 & DOWN & $9.66 \mathrm{E}-05$ \\
\hline NM_003680 & YARS & 2.1 & DOWN & 5.43E-06 \\
\hline NM_000985 & MIT:HsG2227 & 2.1 & DOWN & $1.24 \mathrm{E}-05$ \\
\hline NM_017983 & WIPI1 & 2.1 & DOWN & 2.41E-05 \\
\hline NM_007107 & SSR3 & 2.1 & DOWN & $7.05 \mathrm{E}-05$ \\
\hline AB029551 & RYBP & 2.1 & DOWN & 2.62E-05 \\
\hline NM_021928 & SPCS3 & 2.1 & DOWN & $1.24 \mathrm{E}-04$ \\
\hline NM_018096 & NLE1 & 2.1 & DOWN & $6.74 \mathrm{E}-05$ \\
\hline NM_002930 & RIT2 & 2.1 & DOWN & 6.57E-04 \\
\hline AF121255 & $\mathrm{EIF} 2 \mathrm{C} 2$ & 2.1 & DOWN & 2.87E-04 \\
\hline AK026217 & TTC17 & 2.1 & DOWN & $6.96 \mathrm{E}-06$ \\
\hline NM_031437 & RASSF5 & 2.1 & DOWN & 4.13E-04 \\
\hline BC014527 & $\mathrm{FBXO27}$ & 2.1 & DOWN & 4.82E-05 \\
\hline NM_024580 & EFTUD1 & 2.1 & DOWN & $3.12 \mathrm{E}-04$ \\
\hline NM_006835 & $\mathrm{CCNI}$ & 2.1 & DOWN & $6.63 \mathrm{E}-05$ \\
\hline NM_002507 & NGFR & 2.1 & DOWN & 2.95E-05 \\
\hline AK056817 & NA & 2.1 & DOWN & $1.38 \mathrm{E}-04$ \\
\hline NM_006410 & HTATIP2 & 2.1 & DOWN & $5.29 \mathrm{E}-04$ \\
\hline AJ420461 & QSCN6L1 & 2.1 & DOWN & $1.68 \mathrm{E}-06$ \\
\hline
\end{tabular}




\begin{tabular}{|c|c|c|c|c|}
\hline BC017650 & C1orf201 & 2.1 & DOWN & $2.58 \mathrm{E}-04$ \\
\hline U82319 & C6orf68 & 2.1 & DOWN & 1.16E-04 \\
\hline NM_006513 & SARS & 2.1 & DOWN & $1.09 \mathrm{E}-05$ \\
\hline NM_003875 & GMPS & 2.1 & DOWN & 4.64E-04 \\
\hline NM_016470 & CT111_HUMAN & 2.1 & DOWN & 4.55E-04 \\
\hline AB011100 & KIAA0528 & 2.1 & DOWN & 4.59E-06 \\
\hline NM_018630 & DERL1 & 2.1 & DOWN & $5.96 \mathrm{E}-05$ \\
\hline AK024597 & TRIM25 & 2.1 & DOWN & $1.41 \mathrm{E}-04$ \\
\hline NM_032717 & NP_116106.2 & 2.1 & DOWN & $6.39 \mathrm{E}-06$ \\
\hline NM_017993 & NP_060463.1 & 2.1 & DOWN & $7.64 \mathrm{E}-06$ \\
\hline NM_004337 & OSGIN2 & 2.1 & DOWN & 7.14E-06 \\
\hline NM_004405 & DLX2 & 2.1 & DOWN & $6.69 \mathrm{E}-05$ \\
\hline NM_006164 & NFE2L2 & 2.1 & DOWN & $9.77 \mathrm{E}-06$ \\
\hline NM_014812 & CEP170 & 2.1 & DOWN & 4.04E-05 \\
\hline M12679 & HLA-C & 2.1 & DOWN & $6.10 \mathrm{E}-05$ \\
\hline NM_000638 & VTN & 2.1 & DOWN & $1.69 \mathrm{E}-04$ \\
\hline NM_052888 & LRRC37B & 2.1 & DOWN & 8.31E-07 \\
\hline AB051551 & LRRCC1 & 2.1 & DOWN & $6.31 \mathrm{E}-04$ \\
\hline AF263613 & PNPLA10P & 2.1 & DOWN & 1.44E-05 \\
\hline NM_007214 & SEC63 & 2.1 & DOWN & 4.24E-05 \\
\hline NM_024640 & YRDC & 2.1 & DOWN & $3.12 \mathrm{E}-05$ \\
\hline AK024276 & Q8ND77_HUMAN & 2.1 & DOWN & $2.42 \mathrm{E}-05$ \\
\hline NM_002116 & HLA-H & 2.1 & DOWN & $2.92 \mathrm{E}-04$ \\
\hline NM_013376 & SERTAD1 & 2.1 & DOWN & 2.18E-05 \\
\hline NM_016582 & SLC15A3 & 2.0 & DOWN & 4.26E-04 \\
\hline NM_003904 & ZNF259 & 2.0 & DOWN & $2.59 \mathrm{E}-05$ \\
\hline AK027113 & BCM:bcm714 & 2.0 & DOWN & 2.73E-04 \\
\hline AB067493 & BCM:Q96PY1 & 2.0 & DOWN & 2.54E-04 \\
\hline NM_024052 & C17orf39 & 2.0 & DOWN & $1.30 \mathrm{E}-05$ \\
\hline AB037797 & ARRDC3 & 2.0 & DOWN & 7.86E-04 \\
\hline AL137480 & FNBP4 & 2.0 & DOWN & 1.04E-04 \\
\hline NM_004063 & $\mathrm{CDH} 17$ & 2.0 & DOWN & $6.59 \mathrm{E}-05$ \\
\hline NM_025000 & C2orf37 & 2.0 & DOWN & $7.88 \mathrm{E}-05$ \\
\hline AB046794 & FAM29A & 2.0 & DOWN & 1.19E-04 \\
\hline L21934 & SOAT1 & 2.0 & DOWN & $1.41 \mathrm{E}-04$ \\
\hline NM_015497 & TMEM87A & 2.0 & DOWN & $2.75 \mathrm{E}-04$ \\
\hline BC003353 & MED10 & 2.0 & DOWN & $9.15 \mathrm{E}-04$ \\
\hline NM_006170 & NOL1 & 2.0 & DOWN & 2.88E-04 \\
\hline NM_014811 & KIAA0649 & 2.0 & DOWN & $5.30 \mathrm{E}-06$ \\
\hline NM_003414 & ZNF267 & 2.0 & DOWN & $4.98 \mathrm{E}-04$ \\
\hline AK024858 & LEMD2 & 2.0 & DOWN & $6.41 \mathrm{E}-06$ \\
\hline NM_004865 & TBPL1 & 2.0 & DOWN & $5.61 \mathrm{E}-06$ \\
\hline BC011000 & CDCA5 & 2.0 & DOWN & $1.76 \mathrm{E}-04$ \\
\hline NM_003866 & INPP4B & 2.0 & DOWN & 1.05E-04 \\
\hline NM_005087 & FXR1 & 2.0 & DOWN & $3.31 \mathrm{E}-04$ \\
\hline NM_015946 & PELO & 2.0 & DOWN & $7.64 \mathrm{E}-06$ \\
\hline NM_032842 & NP_116231.2 & 2.0 & DOWN & 4.06E-05 \\
\hline NM_004623 & TTC4 & 2.0 & DOWN & 2.03E-05 \\
\hline NM_017945 & SLC35A5 & 2.0 & DOWN & $2.61 \mathrm{E}-06$ \\
\hline NM_016078 & FAM18B & 2.0 & DOWN & $5.42 \mathrm{E}-04$ \\
\hline NM_003753 & EIF3S7 & 2.0 & DOWN & $1.33 \mathrm{E}-04$ \\
\hline NM_020156 & C1GALT1 & 2.0 & DOWN & $3.19 \mathrm{E}-05$ \\
\hline NM_003659 & AGPS & 2.0 & DOWN & 1.19E-04 \\
\hline AF070674 & BIRC3 & 2.0 & DOWN & 1.15E-04 \\
\hline NM_002874 & RAD23B & 2.0 & UP & $3.01 \mathrm{E}-04$ \\
\hline AB007857 & RUTBC1 & 2.0 & UP & $1.69 \mathrm{E}-04$ \\
\hline AK001536 & C22:AP000525.4 & 2.0 & UP & $1.70 \mathrm{E}-04$ \\
\hline NM_007286 & SYNPO & 2.0 & UP & $5.41 \mathrm{E}-04$ \\
\hline BC010145 & DNAJC4 & 2.0 & UP & $5.65 \mathrm{E}-05$ \\
\hline NM_006319 & CDIPT & 2.0 & UP & $1.54 \mathrm{E}-04$ \\
\hline NM_015544 & TMEM98 & 2.0 & UP & $5.82 \mathrm{E}-06$ \\
\hline AJ420812 & SLC44A1 & 2.0 & UP & $3.32 \mathrm{E}-04$ \\
\hline
\end{tabular}




\begin{tabular}{|c|c|c|c|c|}
\hline NM_024509 & LRFN3 & 2.0 & UP & $3.97 \mathrm{E}-05$ \\
\hline AL080219 & RTCD1 & 2.0 & UP & $7.22 \mathrm{E}-05$ \\
\hline NM_014604 & P2RX5 & 2.0 & UP & 5.87E-04 \\
\hline NM_001972 & ELA2 & 2.0 & UP & $1.22 \mathrm{E}-05$ \\
\hline NM_001904 & CTNNB1 & 2.0 & UP & $3.94 \mathrm{E}-05$ \\
\hline NM_005982 & SIX1 & 2.0 & UP & 7.66E-04 \\
\hline NM_001703 & BAI2 & 2.0 & UP & 4.35E-04 \\
\hline BC000039 & FAM26B & 2.0 & UP & $2.86 \mathrm{E}-05$ \\
\hline NM_014217 & KCNK2 & 2.0 & UP & $2.76 \mathrm{E}-04$ \\
\hline NM_014051 & TMEM14A & 2.0 & UP & $2.35 \mathrm{E}-05$ \\
\hline AK027274 & MARCKS & 2.0 & UP & $2.18 \mathrm{E}-05$ \\
\hline NM_000900 & MGP & 2.1 & UP & $3.64 \mathrm{E}-04$ \\
\hline NM_000380 & XPA & 2.1 & UP & $8.20 \mathrm{E}-04$ \\
\hline NM_000858 & GUK1 & 2.1 & UP & $1.37 \mathrm{E}-04$ \\
\hline AF070641 & ETV1 & 2.1 & UP & 2.42E-04 \\
\hline NM_002695 & POLR2E & 2.1 & UP & 4.91E-05 \\
\hline NM_018347 & CT029_HUMAN & 2.1 & UP & $1.12 \mathrm{E}-04$ \\
\hline NM_018719 & CDCA7L & 2.1 & UP & $1.08 \mathrm{E}-05$ \\
\hline NM_003127 & SPTAN1 & 2.1 & UP & 7.43E-04 \\
\hline AK054707 & ROGDI & 2.1 & UP & 4.49E-06 \\
\hline AK022939 & CEP27 & 2.1 & UP & $3.46 \mathrm{E}-06$ \\
\hline NM_015976 & SNX7 & 2.1 & UP & $2.34 \mathrm{E}-05$ \\
\hline NM_004663 & RAB11A & 2.1 & UP & 7.03E-05 \\
\hline NM_005766 & FARP1 & 2.1 & UP & 3.13E-04 \\
\hline BC007323 & NDUFC2 & 2.1 & UP & 1.19E-04 \\
\hline NM_024863 & TCEAL4 & 2.1 & UP & $1.39 \mathrm{E}-05$ \\
\hline NM_018355 & ZNF415 & 2.1 & UP & $3.84 \mathrm{E}-04$ \\
\hline NM_000093 & COL5A1 & 2.1 & UP & $3.44 \mathrm{E}-06$ \\
\hline AL137535 & C9orf3 & 2.1 & UP & $3.20 \mathrm{E}-04$ \\
\hline NM_002861 & PCYT2 & 2.1 & UP & $5.13 \mathrm{E}-04$ \\
\hline NM_004809 & STOML1 & 2.1 & UP & 7.13E-05 \\
\hline NM_032409 & PINK1 & 2.1 & UP & $3.42 \mathrm{E}-04$ \\
\hline NM_016199 & LSM7 & 2.1 & UP & 4.62E-04 \\
\hline AB046781 & UACA & 2.1 & UP & $1.99 \mathrm{E}-06$ \\
\hline BC008744 & PLEKHQ1 & 2.1 & UP & 4.10E-05 \\
\hline AK057652 & NA & 2.1 & UP & 7.81E-04 \\
\hline NM_014248 & RBX1 & 2.1 & UP & $2.77 \mathrm{E}-05$ \\
\hline NM_021210 & TRAPPC1 & 2.1 & UP & 7.92E-05 \\
\hline BC000977 & ALAD & 2.1 & UP & $1.35 \mathrm{E}-04$ \\
\hline AK023815 & GALNT10 & 2.1 & UP & $2.00 \mathrm{E}-06$ \\
\hline NM_012135 & FAM50B & 2.1 & UP & $3.47 \mathrm{E}-06$ \\
\hline NM_024042 & METRN & 2.1 & UP & $5.72 \mathrm{E}-05$ \\
\hline AK026666 & NP_001026897.1 & 2.1 & UP & 7.34E-05 \\
\hline NM_024833 & ZNF671 & 2.1 & UP & $1.90 \mathrm{E}-04$ \\
\hline NM_000600 & IL6 & 2.1 & UP & 2.33E-04 \\
\hline NM_015392 & NPDC1 & 2.1 & UP & 4.16E-05 \\
\hline NM_022823 & FNDC4 & 2.1 & UP & 4.02E-04 \\
\hline NM_005432 & XRCC3 & 2.1 & UP & 4.77E-05 \\
\hline AK055683 & ZMAT2 & 2.1 & UP & 2.69E-05 \\
\hline NM_001060 & TBXA2R & 2.1 & UP & $3.57 \mathrm{E}-05$ \\
\hline NM_003893 & LDB1 & 2.1 & UP & $3.49 \mathrm{E}-06$ \\
\hline AL049980 & HSD17B12 & 2.1 & UP & $4.38 \mathrm{E}-05$ \\
\hline NM_006848 & CCDC85B & 2.2 & UP & $1.12 \mathrm{E}-04$ \\
\hline NM_016113 & TRPV2 & 2.2 & UP & $2.75 \mathrm{E}-04$ \\
\hline AK054986 & RP5-998N21.2 & 2.2 & UP & $9.44 \mathrm{E}-06$ \\
\hline NM_032601 & MCEE & 2.2 & UP & $9.75 \mathrm{E}-07$ \\
\hline NM_021570 & BARX1 & 2.2 & UP & $3.88 \mathrm{E}-05$ \\
\hline NM_000859 & HMGCR & 2.2 & UP & 7.75E-04 \\
\hline AF156100 & HMCN1 & 2.2 & UP & $2.57 \mathrm{E}-04$ \\
\hline NM_006810 & PDIA5 & 2.2 & UP & $3.40 \mathrm{E}-05$ \\
\hline NM_004638 & BAT2 & 2.2 & UP & $2.14 \mathrm{E}-06$ \\
\hline NM_006412 & AGPAT2 & 2.2 & UP & $1.86 \mathrm{E}-05$ \\
\hline
\end{tabular}




\begin{tabular}{|c|c|c|c|c|}
\hline NM_024825 & PODNL1 & 2.2 & UP & $1.32 \mathrm{E}-05$ \\
\hline BC018118 & ARHGAP1 & 2.2 & UP & $1.94 \mathrm{E}-05$ \\
\hline AB037823 & CHGUT_HUMAN & 2.2 & UP & $2.48 \mathrm{E}-04$ \\
\hline AK056039 & NA & 2.2 & UP & $5.90 \mathrm{E}-05$ \\
\hline AK025071 & SPTBN1 & 2.2 & UP & $3.72 \mathrm{E}-06$ \\
\hline NM_016206 & BCM:NM_016206 & 2.2 & UP & 3.23E-04 \\
\hline NM_012317 & LDOC1 & 2.2 & UP & 4.84E-05 \\
\hline NM_006074 & TRIM22 & 2.2 & UP & $9.73 \mathrm{E}-05$ \\
\hline BC009566 & TAF9B & 2.2 & UP & $1.04 \mathrm{E}-04$ \\
\hline AK056499 & PRICKLE1 & 2.2 & UP & $1.19 \mathrm{E}-04$ \\
\hline NM_005566 & LDHA & 2.2 & UP & 1.77E-04 \\
\hline NM_005009 & NME4 & 2.2 & UP & $1.27 \mathrm{E}-06$ \\
\hline NM_014324 & AMACR & 2.3 & UP & $1.51 \mathrm{E}-05$ \\
\hline AJ272212 & PSKH1 & 2.3 & UP & $1.25 \mathrm{E}-06$ \\
\hline NM_003491 & ARD1A & 2.3 & UP & $3.16 \mathrm{E}-06$ \\
\hline AK021925 & SLC41A3 & 2.3 & UP & $1.94 \mathrm{E}-05$ \\
\hline U60873 & $\mathrm{NA}$ & 2.3 & UP & $5.62 \mathrm{E}-04$ \\
\hline NM_000138 & FBN1 & 2.3 & UP & $8.46 \mathrm{E}-04$ \\
\hline AK025371 & ASAHL & 2.3 & UP & 7.97E-05 \\
\hline AK055506 & VGCNL1 & 2.3 & UP & $3.65 \mathrm{E}-05$ \\
\hline NM_000107 & DDB2 & 2.3 & UP & $2.44 \mathrm{E}-06$ \\
\hline NM_003528 & HIST2H2BE & 2.3 & UP & $2.06 \mathrm{E}-04$ \\
\hline AK056513 & NP_653327.1 & 2.3 & UP & $7.02 \mathrm{E}-06$ \\
\hline NM_005566 & LDHA & 2.3 & UP & $7.22 \mathrm{E}-05$ \\
\hline AK000672 & FAM82B & 2.3 & UP & 4.62E-04 \\
\hline NM_021643 & TRIB2 & 2.3 & UP & $1.80 \mathrm{E}-05$ \\
\hline NM_005111 & CRYZL1 & 2.3 & UP & $3.80 \mathrm{E}-04$ \\
\hline NM_024062 & VANGL1 & 2.3 & UP & 4.66E-05 \\
\hline NM_003492 & CXorf12 & 2.3 & UP & $1.10 \mathrm{E}-05$ \\
\hline NM_003634 & NIPSNAP1 & 2.3 & UP & $2.74 \mathrm{E}-05$ \\
\hline AB011540 & LRP4 & 2.3 & UP & 4.61E-04 \\
\hline NM_015373 & PGEA1 & 2.3 & UP & $1.41 \mathrm{E}-05$ \\
\hline AK055699 & LYPD6 & 2.3 & UP & $5.69 \mathrm{E}-05$ \\
\hline AK023619 & CRIM1 & 2.3 & UP & $1.10 \mathrm{E}-05$ \\
\hline NM_004538 & NAP1L3 & 2.3 & UP & 4.45E-05 \\
\hline NM_032262 & NP_115638.1 & 2.3 & UP & $1.78 \mathrm{E}-04$ \\
\hline AK021851 & NA & 2.3 & UP & 3.45E-05 \\
\hline NM_001611 & ACP5 & 2.3 & UP & 2.63E-04 \\
\hline NM_001215 & CA6 & 2.3 & UP & $4.77 \mathrm{E}-04$ \\
\hline NM_003118 & SPARC & 2.4 & UP & 8.94E-04 \\
\hline NM_000476 & AK1 & 2.4 & UP & $3.37 \mathrm{E}-06$ \\
\hline AK027351 & RHOJ & 2.4 & UP & $7.50 \mathrm{E}-04$ \\
\hline NM_004528 & MGST3 & 2.4 & UP & $1.28 \mathrm{E}-04$ \\
\hline NM_001336 & CTSZ & 2.4 & UP & $9.43 \mathrm{E}-05$ \\
\hline AL359052 & ITGBL1 & 2.4 & UP & 4.39E-04 \\
\hline NM_006822 & RAB40B & 2.4 & UP & $1.86 \mathrm{E}-05$ \\
\hline NM_004642 & CDK2AP1 & 2.4 & UP & $1.38 \mathrm{E}-06$ \\
\hline AK055294 & SHROOM3 & 2.4 & UP & $3.31 \mathrm{E}-05$ \\
\hline AK021486 & BMP5 & 2.4 & UP & $2.78 \mathrm{E}-04$ \\
\hline AK057566 & ANKRD13D & 2.4 & UP & $2.45 \mathrm{E}-07$ \\
\hline NM_003516 & HIST2H2AA3 & 2.4 & UP & $4.98 \mathrm{E}-04$ \\
\hline NM_002452 & NUDT1 & 2.4 & UP & $1.00 \mathrm{E}-04$ \\
\hline AK056203 & LRSAM1 & 2.4 & UP & $1.20 \mathrm{E}-04$ \\
\hline NM_016429 & COPZ2 & 2.4 & UP & $9.87 \mathrm{E}-05$ \\
\hline NM_017702 & NP_997397.1 & 2.4 & UP & $8.50 \mathrm{E}-06$ \\
\hline NM_025149 & NP_079425.2 & 2.4 & UP & $1.08 \mathrm{E}-04$ \\
\hline NM_003528 & HIST2H2BE & 2.4 & UP & 1.07E-04 \\
\hline NM_002744 & PRKCZ & 2.4 & UP & $1.61 \mathrm{E}-04$ \\
\hline AK057356 & GGN & 2.4 & UP & 4.36E-05 \\
\hline NM_005767 & P2RY5 & 2.5 & UP & 4.16E-04 \\
\hline BC013049 & MRRF & 2.5 & UP & 3.87E-06 \\
\hline NM_003528 & HIST2H2BE & 2.5 & UP & $3.76 \mathrm{E}-04$ \\
\hline
\end{tabular}




\begin{tabular}{|c|c|c|c|c|}
\hline AB011539 & MEGF6 & 2.5 & UP & 4.33E-05 \\
\hline NM_022783 & DEPDC6 & 2.5 & UP & 4.34E-04 \\
\hline NM_002863 & PYGL & 2.5 & UP & 2.79E-05 \\
\hline NM_003528 & HIST2H2BE & 2.5 & UP & $2.38 \mathrm{E}-04$ \\
\hline NM_005418 & ST5 & 2.5 & UP & $5.99 \mathrm{E}-06$ \\
\hline AK025953 & MYLK & 2.5 & UP & $5.23 \mathrm{E}-04$ \\
\hline M62896 & NA & 2.5 & UP & $1.24 \mathrm{E}-04$ \\
\hline NM_031302 & GLT8D2 & 2.5 & UP & 8.59E-08 \\
\hline AK025627 & MIT:HsG2201 & 2.5 & UP & 4.96E-06 \\
\hline AK056473 & FAM33A & 2.5 & UP & 3.84E-05 \\
\hline NM_003528 & HIST2H2BE & 2.5 & UP & 2.10E-04 \\
\hline BC012170 & C3orf54 & 2.5 & UP & 7.64E-06 \\
\hline NM_005566 & LDHA & 2.5 & UP & 3.57E-05 \\
\hline NM_021923 & FGFRL1 & 2.5 & UP & $1.51 \mathrm{E}-04$ \\
\hline NM_002725 & PRELP & 2.5 & UP & $2.83 \mathrm{E}-04$ \\
\hline NM_003250 & THRA & 2.6 & UP & $9.81 \mathrm{E}-07$ \\
\hline NM_005101 & ISG15 & 2.6 & UP & $5.42 \mathrm{E}-04$ \\
\hline NM_003528 & HIST2H2BE & 2.6 & UP & 7.71E-05 \\
\hline AL133084 & PGBD3 & 2.6 & UP & $6.87 \mathrm{E}-05$ \\
\hline AY008268 & SAR1A & 2.6 & UP & 1.73E-05 \\
\hline AK027191 & NA & 2.6 & UP & 7.15E-05 \\
\hline NM_001822 & CHN1 & 2.6 & UP & 2.43E-06 \\
\hline NM_000177 & GSN & 2.6 & UP & $6.57 \mathrm{E}-06$ \\
\hline AK055808 & NP_001070148.1 & 2.6 & UP & $1.99 \mathrm{E}-05$ \\
\hline NM_033138 & CALD1 & 2.6 & UP & 7.03E-05 \\
\hline NM_003528 & HIST2H2BE & 2.6 & UP & 3.35E-05 \\
\hline NM_057161 & KLHDC3 & 2.6 & UP & 4.30E-07 \\
\hline NM_005566 & LDHA & 2.6 & UP & $6.45 \mathrm{E}-05$ \\
\hline NM_005469 & АCOT8 & 2.6 & UP & 7.98E-05 \\
\hline AF380356 & $X G$ & 2.6 & UP & 2.45E-04 \\
\hline NM_005572 & LMNA & 2.6 & UP & $9.96 \mathrm{E}-06$ \\
\hline NM_003246 & THBS1 & 2.6 & UP & $6.37 \mathrm{E}-07$ \\
\hline NM_003956 & $\mathrm{CH} 25 \mathrm{H}$ & 2.6 & UP & 2.75E-04 \\
\hline NM_004701 & CCNB2 & 2.6 & UP & 3.76E-04 \\
\hline NM_016332 & SEPX1 & 2.6 & UP & 4.35E-07 \\
\hline BC012625 & PPP1R3C & 2.6 & UP & 2.85E-07 \\
\hline AB011110 & RASA4 & 2.6 & UP & 4.39E-07 \\
\hline NM_032800 & C1orf198 & 2.7 & UP & 1.77E-04 \\
\hline NM_003528 & HIST2H2BE & 2.7 & UP & 7.01E-05 \\
\hline U97105 & DPYSL2 & 2.7 & UP & 7.48E-07 \\
\hline NM_002966 & $\mathrm{S} 100 \mathrm{~A} 10$ & 2.7 & UP & 3.33E-04 \\
\hline NM_032291 & SGIP1 & 2.7 & UP & $2.56 \mathrm{E}-05$ \\
\hline NM_001548 & IFIT1 & 2.7 & UP & 4.86E-05 \\
\hline NM_052969 & RPL39L & 2.7 & UP & $3.86 \mathrm{E}-06$ \\
\hline NM_021874 & CDC25B & 2.7 & UP & $3.71 \mathrm{E}-05$ \\
\hline AL080111 & NEK7 & 2.7 & UP & 7.44E-04 \\
\hline NM_000820 & GAS6 & 2.7 & UP & $3.31 \mathrm{E}-05$ \\
\hline NM_022343 & GAPR1_HUMAN & 2.7 & UP & 1.03E-06 \\
\hline NM_000077 & CDKN2A & 2.7 & UP & 4.36E-05 \\
\hline AK026164 & MYL6 & 2.7 & UP & 4.76E-08 \\
\hline AK057333 & C11orf66 & 2.7 & UP & 7.04E-05 \\
\hline BC013438 & Q96D13_HUMAN & 2.8 & UP & $5.41 \mathrm{E}-06$ \\
\hline NM_005491 & CXorf6 & 2.8 & UP & 4.91E-08 \\
\hline NM_002313 & ABLIM1 & 2.8 & UP & 1.99E-04 \\
\hline NM_018953 & HOXC5 & 2.8 & UP & $6.37 \mathrm{E}-06$ \\
\hline AB051459 & DJBP_HUMAN & 2.8 & UP & 1.15E-06 \\
\hline NM_006579 & EBP & 2.8 & UP & 2.01E-05 \\
\hline AL049957 & CD59 & 2.8 & UP & 2.25E-06 \\
\hline NM_016471 & ORMDL3 & 2.8 & UP & 1.06E-06 \\
\hline NM_002413 & MGST2 & 2.8 & UP & 4.33E-07 \\
\hline NM_005423 & TFF2 & 2.9 & UP & $2.84 \mathrm{E}-05$ \\
\hline NM_003070 & SMARCA2 & 2.9 & UP & $5.66 \mathrm{E}-06$ \\
\hline
\end{tabular}




\begin{tabular}{|c|c|c|c|c|}
\hline AK023464 & BCM:bcm899 & 2.9 & UP & $8.56 \mathrm{E}-07$ \\
\hline AL050137 & OLFML2B & 2.9 & UP & $2.42 \mathrm{E}-08$ \\
\hline AK026717 & SEPW1 & 2.9 & UP & 4.33E-06 \\
\hline NM_002609 & PDGFRB & 2.9 & UP & $5.24 \mathrm{E}-07$ \\
\hline NM_020166 & MCCC1 & 2.9 & UP & $1.97 \mathrm{E}-06$ \\
\hline NM_005566 & LDHA & 2.9 & UP & 1.69E-06 \\
\hline X68742 & PELO & 2.9 & UP & 6.63E-04 \\
\hline NM_014583 & LMCD1 & 2.9 & UP & $2.78 \mathrm{E}-04$ \\
\hline NM_053025 & MYLK & 2.9 & UP & $1.05 \mathrm{E}-04$ \\
\hline NM_003528 & HIST2H2BE & 2.9 & UP & $7.71 \mathrm{E}-05$ \\
\hline NM_004765 & $\mathrm{BCL} 7 \mathrm{C}$ & 2.9 & UP & 8.87E-07 \\
\hline NM_006986 & MAGED1 & 2.9 & UP & 1.99E-08 \\
\hline AF043897 & hsa-mir-23b & 2.9 & UP & $5.59 \mathrm{E}-07$ \\
\hline AB046764 & NBEA & 3.0 & UP & $1.69 \mathrm{E}-05$ \\
\hline AB037750 & SORCS2 & 3.0 & UP & $8.10 \mathrm{E}-06$ \\
\hline L10374 & Q5W0J2_HUMAN & 3.0 & UP & 3.46E-06 \\
\hline NM_000022 & ADA & 3.0 & UP & 2.36E-04 \\
\hline AK024192 & RBPMS & 3.0 & UP & $3.11 \mathrm{E}-04$ \\
\hline NM_000163 & GHR & 3.0 & UP & $6.63 \mathrm{E}-05$ \\
\hline AK021874 & TGFB2 & 3.0 & UP & $6.08 \mathrm{E}-07$ \\
\hline NM_030786 & SYNC1 & 3.0 & UP & $4.72 \mathrm{E}-08$ \\
\hline AF090693 & CUGBP2 & 3.1 & UP & $6.71 \mathrm{E}-04$ \\
\hline AK022222 & CALD1 & 3.1 & UP & 2.13E-04 \\
\hline NM_001845 & COL4A1 & 3.1 & UP & $3.95 \mathrm{E}-05$ \\
\hline NM_003512 & HIST1H2AC & 3.1 & UP & $3.42 \mathrm{E}-06$ \\
\hline NM_003247 & THBS2 & 3.1 & UP & $5.41 \mathrm{E}-04$ \\
\hline NM_003289 & TPM2 & 3.1 & UP & $3.36 \mathrm{E}-05$ \\
\hline AL359558 & MCC & 3.1 & UP & 2.97E-07 \\
\hline NM_000943 & PPIC & 3.1 & UP & $1.12 \mathrm{E}-08$ \\
\hline BC015134 & THBS1 & 3.1 & UP & $1.02 \mathrm{E}-07$ \\
\hline NM_022129 & MAWBP_HUMAN & 3.2 & UP & 4.51E-06 \\
\hline NM_054033 & FKBP1B & 3.2 & UP & 1.47E-06 \\
\hline NM_003280 & TNNC1 & 3.2 & UP & $1.97 \mathrm{E}-05$ \\
\hline NM_032348 & MXRA8 & 3.2 & UP & 1.27E-07 \\
\hline NM_014867 & KBTBD11 & 3.2 & UP & $5.31 \mathrm{E}-07$ \\
\hline NM_016557 & NA & 3.3 & UP & 2.19E-04 \\
\hline NM_001512 & GSTA4 & 3.3 & UP & 7.09E-08 \\
\hline AB046796 & K1576_HUMAN & 3.3 & UP & 1.83E-04 \\
\hline NM_004512 & GALT & 3.3 & UP & $1.82 \mathrm{E}-06$ \\
\hline NM_021730 & Q96AM0_HUMAN & 3.4 & UP & 8.15E-09 \\
\hline AY039760 & ATP6V1G3 & 3.4 & UP & 2.70E-06 \\
\hline NM_004409 & DMPK & 3.4 & UP & $1.02 \mathrm{E}-08$ \\
\hline AF245505 & MXRA5 & 3.4 & UP & $3.56 \mathrm{E}-05$ \\
\hline NM_002775 & HTRA1 & 3.4 & UP & $1.56 \mathrm{E}-06$ \\
\hline NM_012134 & LMOD1 & 3.4 & UP & 2.69E-05 \\
\hline NM_002048 & GAS1 & 3.4 & UP & $1.79 \mathrm{E}-05$ \\
\hline NM_000253 & MTTP & 3.5 & UP & $1.31 \mathrm{E}-07$ \\
\hline NM_001792 & $\mathrm{CDH} 2$ & 3.5 & UP & 2.01E-05 \\
\hline NM_000393 & COL5A2 & 3.5 & UP & $5.24 \mathrm{E}-07$ \\
\hline AK021982 & DYM & 3.5 & UP & 7.59E-08 \\
\hline BC013971 & HOXA10 & 3.5 & UP & $1.32 \mathrm{E}-07$ \\
\hline AB032947 & CADPS & 3.5 & UP & $1.71 \mathrm{E}-07$ \\
\hline NM_018936 & PCDHB2 & 3.5 & UP & 4.07E-04 \\
\hline AK057501 & CMTM2 & 3.5 & UP & 4.21E-07 \\
\hline NM_030915 & LBH & 3.6 & UP & $2.54 \mathrm{E}-04$ \\
\hline NM_032603 & LOXL3 & 3.7 & UP & $9.96 \mathrm{E}-08$ \\
\hline NM_000090 & COL3A1 & 3.7 & UP & $1.00 \mathrm{E}-06$ \\
\hline AK001007 & GRAMD3 & 3.7 & UP & $5.21 \mathrm{E}-05$ \\
\hline NM_005195 & Q504X4_HUMAN & 3.7 & UP & 1.39E-04 \\
\hline NM_004265 & FADS2 & 3.8 & UP & 7.45E-04 \\
\hline NM_020404 & CD248 & 3.8 & UP & 2.00E-09 \\
\hline NM_004772 & C5orf13 & 4.1 & UP & $8.15 \mathrm{E}-08$ \\
\hline
\end{tabular}




\begin{tabular}{|c|c|c|c|c|}
\hline AK025346 & NA & 4.3 & UP & $7.64 \mathrm{E}-06$ \\
\hline NM_052880 & NP_443112.2 & 4.3 & UP & $5.66 \mathrm{E}-06$ \\
\hline NM_014298 & QPRT & 4.3 & UP & $5.29 \mathrm{E}-04$ \\
\hline NM_002527 & NTF3 & 4.4 & UP & $4.72 \mathrm{E}-08$ \\
\hline NM_004415 & DSP & 4.4 & UP & $9.85 \mathrm{E}-06$ \\
\hline AK057328 & NA & 4.5 & UP & $1.12 \mathrm{E}-07$ \\
\hline AF444143 & SPG3A & 4.5 & UP & $1.06 \mathrm{E}-05$ \\
\hline AK058001 & DENND3 & 4.5 & UP & 4.46E-07 \\
\hline NM_032623 & NP_116012.2 & 4.6 & UP & $5.69 \mathrm{E}-05$ \\
\hline NM_014762 & DHCR24 & 4.6 & UP & 2.42E-05 \\
\hline NM_023927 & GRAMD3 & 4.6 & UP & $1.59 \mathrm{E}-07$ \\
\hline AL137734 & $\mathrm{NA}$ & 4.6 & UP & $3.82 \mathrm{E}-06$ \\
\hline NM_031289 & GSG1 & 4.8 & UP & $5.19 \mathrm{E}-06$ \\
\hline NM_002961 & S100A4 & 4.8 & UP & $1.35 \mathrm{E}-05$ \\
\hline NM_023067 & FOXL2 & 4.8 & UP & $9.10 \mathrm{E}-05$ \\
\hline NM_004881 & TP53I3 & 4.8 & UP & $3.12 \mathrm{E}-11$ \\
\hline NM_002982 & CCL2 & 4.9 & UP & $4.91 \mathrm{E}-08$ \\
\hline AF213459 & EPHA3 & 5.0 & UP & $1.31 \mathrm{E}-06$ \\
\hline NM_000147 & FUCA1 & 5.1 & UP & 2.24E-08 \\
\hline NM_001864 & COX7A1 & 5.2 & UP & $9.50 \mathrm{E}-08$ \\
\hline NM_005584 & MAB21L1 & 5.2 & UP & $8.15 \mathrm{E}-08$ \\
\hline BC012486 & KRA24_HUMAN & 5.2 & UP & $5.97 \mathrm{E}-05$ \\
\hline AJ245539 & GALNT5 & 5.4 & UP & 2.79E-10 \\
\hline AK055249 & UCHL1 & 5.6 & UP & $5.82 \mathrm{E}-10$ \\
\hline NM_001175 & ARHGDIB & 5.6 & UP & $1.56 \mathrm{E}-06$ \\
\hline NM_000346 & sox9 & 5.7 & UP & 1.47E-05 \\
\hline AL137678 & C20orf50 & 6.0 & UP & $3.10 \mathrm{E}-08$ \\
\hline NM_004181 & UCHL1 & 6.1 & UP & 7.45E-10 \\
\hline NM_003783 & B3GALT2 & 6.1 & UP & $3.86 \mathrm{E}-05$ \\
\hline AK002039 & MRVI1 & 6.2 & UP & $5.69 \mathrm{E}-05$ \\
\hline NM_004335 & BST2 & 6.7 & UP & $1.46 \mathrm{E}-06$ \\
\hline NM_030967 & KRTAP1-3 & 7.2 & UP & $1.31 \mathrm{E}-06$ \\
\hline NM_000095 & COMP & 7.5 & UP & $7.59 \mathrm{E}-06$ \\
\hline NM_000088 & COL1A1 & 7.7 & UP & $1.02 \mathrm{E}-07$ \\
\hline NM_004669 & CLIC3 & 8.5 & UP & 4.36E-07 \\
\hline NM_000681 & ADRA2A & 8.9 & UP & $2.88 \mathrm{E}-08$ \\
\hline NM_001562 & IL18 & 9.1 & UP & 8.13E-08 \\
\hline NM_020169 & LXN & 9.1 & UP & $2.16 \mathrm{E}-09$ \\
\hline NM_006308 & HSPB3 & 10.3 & UP & 5.95E-08 \\
\hline NM_002276 & KRT19 & 10.6 & UP & 7.14E-08 \\
\hline AK022198 & GALNT5 & 12.6 & UP & 1.13E-09 \\
\hline AB032953 & ODZ2 & 13.3 & UP & $1.38 \mathrm{E}-11$ \\
\hline NM_000089 & COL1A2 & 13.9 & UP & $3.12 \mathrm{E}-11$ \\
\hline AB067499 & Q96PX6_HUMAN & 14.4 & UP & $8.31 \mathrm{E}-07$ \\
\hline AK021484 & SYNPO2 & 22.0 & UP & 4.44E-08 \\
\hline
\end{tabular}


Supplementary table S2: Significantly differentially expressed genes for the 97 versus 0 comparison

\begin{tabular}{|c|c|c|c|c|}
\hline Genbank accession No. & Gene symbol & Fold-change & Direction & $\mathrm{p}$-value \\
\hline NM_000799 & EPO & 67.7 & DOWN & $5.94 \mathrm{E}-11$ \\
\hline NM_002155 & HSPA6 & 52.1 & DOWN & $2.14 \mathrm{E}-11$ \\
\hline NM_002421 & MMP1 & 38.8 & DOWN & $3.82 \mathrm{E}-09$ \\
\hline NM_004864 & GDF15 & 37.3 & DOWN & $1.29 \mathrm{E}-08$ \\
\hline NM_000963 & PTGS2 & 32.4 & DOWN & $2.75 \mathrm{E}-09$ \\
\hline NM_005345 & HSPA1A & 28.8 & DOWN & $1.87 \mathrm{E}-11$ \\
\hline NM_005345 & HSPA1A & 28.3 & DOWN & $5.88 \mathrm{E}-12$ \\
\hline NM_005345 & HSPA1A & 27.9 & DOWN & $5.88 \mathrm{E}-12$ \\
\hline NM_005345 & HSPA1A & 27.7 & DOWN & 1.87E-11 \\
\hline NM_005345 & HSPA1A & 27.7 & DOWN & $5.88 \mathrm{E}-12$ \\
\hline NM_005345 & HSPA1A & 27.6 & DOWN & $4.84 \mathrm{E}-11$ \\
\hline NM_005345 & HSPA1A & 27.5 & DOWN & 2.14E-11 \\
\hline NM_005345 & HSPA1A & 27.1 & DOWN & $4.84 \mathrm{E}-11$ \\
\hline NM_005345 & HSPA1A & 26.2 & DOWN & $5.88 \mathrm{E}-12$ \\
\hline NM_005345 & HSPA1A & 26.1 & DOWN & $4.55 \mathrm{E}-11$ \\
\hline NM_005345 & HSPA1A & 26.1 & DOWN & $5.88 \mathrm{E}-12$ \\
\hline NM_005345 & HSPA1A & 25.9 & DOWN & $5.94 \mathrm{E}-11$ \\
\hline NM_005345 & HSPA1A & 25.4 & DOWN & $5.88 \mathrm{E}-12$ \\
\hline NM_005345 & HSPA1A & 25.3 & DOWN & $4.84 \mathrm{E}-11$ \\
\hline NM_021158 & TRIB3 & 23.9 & DOWN & $1.57 \mathrm{E}-08$ \\
\hline NM_005345 & HSPA1A & 23.6 & DOWN & 2.14E-11 \\
\hline NM_005345 & HSPA1A & 23.1 & DOWN & 7.36E-11 \\
\hline NM_005346 & HSPA1B & 23.0 & DOWN & $1.18 \mathrm{E}-10$ \\
\hline NM_005345 & HSPA1A & 22.8 & DOWN & 2.14E-11 \\
\hline NM_002220 & ITPKA & 20.1 & DOWN & $9.52 \mathrm{E}-11$ \\
\hline NM_022842 & CDCP1 & 19.9 & DOWN & 1.47E-09 \\
\hline NM_004165 & RRAD & 19.3 & DOWN & $9.90 \mathrm{E}-11$ \\
\hline NM_002727 & PRG1 & 18.4 & DOWN & $6.91 \mathrm{E}-10$ \\
\hline NM_002923 & RGS2 & 18.0 & DOWN & $6.20 \mathrm{E}-08$ \\
\hline NM_005252 & FOS & 18.0 & DOWN & $5.09 \mathrm{E}-10$ \\
\hline NM_032621 & BEX2 & 17.3 & DOWN & $3.46 \mathrm{E}-11$ \\
\hline NM_002394 & SLC3A2 & 15.8 & DOWN & $3.46 \mathrm{E}-11$ \\
\hline BC012321 & ARC & 13.8 & DOWN & $3.49 \mathrm{E}-10$ \\
\hline NM_001353 & AKR1C1 & 11.6 & DOWN & $5.45 \mathrm{E}-07$ \\
\hline NM_017947 & MOCOS & 11.5 & DOWN & 4.91E-06 \\
\hline NM_012328 & DNAJB9 & 11.2 & DOWN & 1.46E-10 \\
\hline NM_006145 & DNAJB1 & 11.2 & DOWN & $4.84 \mathrm{E}-11$ \\
\hline NM_001533 & HNRPL & 10.5 & DOWN & $2.44 \mathrm{E}-10$ \\
\hline NM_007115 & TNFAIP6 & 10.3 & DOWN & $3.09 \mathrm{E}-07$ \\
\hline NM_001945 & HBEGF & 10.3 & DOWN & $2.12 \mathrm{E}-07$ \\
\hline NM_000640 & IL13RA2 & 10.2 & DOWN & $8.84 \mathrm{E}-08$ \\
\hline NM_002133 & HMOX1 & 9.8 & DOWN & 3.27E-07 \\
\hline NM_021127 & PMAIP1 & 9.7 & DOWN & $2.16 \mathrm{E}-07$ \\
\hline NM_001902 & CTH & 9.6 & DOWN & $3.69 \mathrm{E}-10$ \\
\hline AB050476 & LHX8 & 9.5 & DOWN & $8.59 \mathrm{E}-10$ \\
\hline BC017001 & SCRT2 & 9.4 & DOWN & $4.16 \mathrm{E}-08$ \\
\hline NM_005347 & HSPA5 & 9.3 & DOWN & 7.37E-06 \\
\hline NM_001451 & FOXF1 & 9.2 & DOWN & 4.19E-08 \\
\hline NM_007289 & MME & 9.1 & DOWN & $2.13 \mathrm{E}-06$ \\
\hline NM_005532 & $\mathrm{IF|} 27$ & 8.9 & DOWN & $6.41 \mathrm{E}-04$ \\
\hline NM_002837 & PTPRB & 8.8 & DOWN & $5.82 \mathrm{E}-08$ \\
\hline NM_005098 & MSC & 8.8 & DOWN & $2.50 \mathrm{E}-08$ \\
\hline NM_000361 & THBD & 8.6 & DOWN & 7.69E-09 \\
\hline NM_000804 & FOLR3 & 8.6 & DOWN & 4.54E-09 \\
\hline AF438313 & TMEM158 & 8.2 & DOWN & $8.53 \mathrm{E}-08$ \\
\hline NM_004862 & LITAF & 8.0 & DOWN & 4.83E-07 \\
\hline NM_013370 & OSGIN1 & 7.7 & DOWN & $2.33 \mathrm{E}-08$ \\
\hline NM_004083 & DDIT3 & 7.7 & DOWN & $1.24 \mathrm{E}-08$ \\
\hline NM_004398 & DDX10 & 7.6 & DOWN & $1.29 \mathrm{E}-08$ \\
\hline
\end{tabular}




\begin{tabular}{|c|c|c|c|c|}
\hline NM_002479 & MYOG & 7.5 & DOWN & $1.78 \mathrm{E}-07$ \\
\hline NM_006010 & ARMET & 7.4 & DOWN & 2.26E-07 \\
\hline NM_007036 & ESM1 & 7.4 & DOWN & 1.40E-05 \\
\hline NM_016084 & RASD1 & 7.3 & DOWN & $2.90 \mathrm{E}-08$ \\
\hline AK001031 & TBX2 & 7.2 & DOWN & $1.02 \mathrm{E}-08$ \\
\hline NM_003364 & UPP1 & 7.1 & DOWN & 3.05E-09 \\
\hline NM_017723 & NP_060193.2 & 7.0 & DOWN & $1.71 \mathrm{E}-04$ \\
\hline NM_006636 & MTHFD2 & 7.0 & DOWN & 4.50E-10 \\
\hline BC016658 & E2F7 & 6.8 & DOWN & $1.25 \mathrm{E}-07$ \\
\hline NM_003038 & SLC1A4 & 6.6 & DOWN & 2.21E-10 \\
\hline AL390153 & MBD3 & 6.5 & DOWN & $6.44 \mathrm{E}-04$ \\
\hline NM_020038 & ABCC3 & 6.5 & DOWN & 7.63E-07 \\
\hline NM_030674 & SLC38A1 & 6.3 & DOWN & 1.56E-07 \\
\hline NM_006389 & HYOU1 & 6.3 & DOWN & $6.72 \mathrm{E}-06$ \\
\hline AL133096 & DNAJA4 & 6.1 & DOWN & $5.24 \mathrm{E}-07$ \\
\hline NM_006470 & TRIM16 & 6.1 & DOWN & $1.26 \mathrm{E}-06$ \\
\hline NM_014751 & MTSS1 & 6.0 & DOWN & 5.50E-07 \\
\hline NM_004362 & CLGN & 6.0 & DOWN & $3.58 \mathrm{E}-08$ \\
\hline NM_001673 & ASNS & 5.9 & DOWN & $6.91 \mathrm{E}-10$ \\
\hline NM_022044 & SDF2L1 & 5.9 & DOWN & 2.66E-08 \\
\hline NM_006732 & FOSB & 5.8 & DOWN & $3.10 \mathrm{E}-09$ \\
\hline NM_006528 & TFPI2 & 5.8 & DOWN & $4.69 \mathrm{E}-06$ \\
\hline NM_032691 & NA & 5.8 & DOWN & 1.25E-09 \\
\hline NM_003330 & Q6YNQ1_HUMAN & 5.7 & DOWN & 1.69E-05 \\
\hline NM_014331 & SLC7A11 & 5.6 & DOWN & $2.60 \mathrm{E}-08$ \\
\hline NM_005402 & RALA & 5.5 & DOWN & $9.32 \mathrm{E}-08$ \\
\hline NM_006644 & HSPH1 & 5.5 & DOWN & $1.70 \mathrm{E}-08$ \\
\hline BC007359 & RTTN & 5.5 & DOWN & $1.94 \mathrm{E}-08$ \\
\hline NM_019058 & DDIT4 & 5.4 & DOWN & $3.58 \mathrm{E}-09$ \\
\hline NM_004419 & DUSP5 & 5.4 & DOWN & $1.47 \mathrm{E}-05$ \\
\hline NM_000584 & IL8 & 5.4 & DOWN & $6.61 \mathrm{E}-09$ \\
\hline M77140 & GAL & 5.4 & DOWN & $1.36 \mathrm{E}-05$ \\
\hline NM_001498 & GCLC & 5.4 & DOWN & 1.23E-09 \\
\hline NM_004024 & ATF3 & 5.4 & DOWN & $5.72 \mathrm{E}-08$ \\
\hline NM_005985 & SNAl1 & 5.4 & DOWN & $1.19 \mathrm{E}-05$ \\
\hline AF101051 & CLDN1 & 5.4 & DOWN & $3.02 \mathrm{E}-04$ \\
\hline NM_020299 & AKR1B10 & 5.4 & DOWN & $1.59 \mathrm{E}-05$ \\
\hline L24498 & GADD45A & 5.3 & DOWN & $5.68 \mathrm{E}-06$ \\
\hline NM_004095 & EIF4EBP1 & 5.3 & DOWN & $1.31 \mathrm{E}-08$ \\
\hline BC016285 & PRKACB & 5.3 & DOWN & $6.63 \mathrm{E}-09$ \\
\hline NM_015675 & GADD45B & 5.3 & DOWN & $2.31 \mathrm{E}-08$ \\
\hline NM_002061 & GCLM & 5.3 & DOWN & 1.43E-08 \\
\hline NM_022743 & SMYD3 & 5.2 & DOWN & 7.30E-07 \\
\hline AB018289 & NP_056002.1 & 5.2 & DOWN & $1.78 \mathrm{E}-04$ \\
\hline NM_000231 & SGCG & 5.2 & DOWN & $5.60 \mathrm{E}-06$ \\
\hline NM_000071 & CBS & 5.2 & DOWN & $6.90 \mathrm{E}-06$ \\
\hline NM_003655 & CBX4 & 5.2 & DOWN & 1.62E-06 \\
\hline NM_000271 & NPC1 & 5.1 & DOWN & $2.54 \mathrm{E}-09$ \\
\hline AF007152 & ABHD3 & 5.1 & DOWN & 1.46E-10 \\
\hline NM_003582 & DYRK3 & 5.1 & DOWN & $1.10 \mathrm{E}-05$ \\
\hline NM_003662 & PIR & 5.1 & DOWN & $1.82 \mathrm{E}-06$ \\
\hline NM_052966 & NIBA_HUMAN & 5.0 & DOWN & 2.07E-06 \\
\hline NM_001793 & $\mathrm{CDH} 3$ & 5.0 & DOWN & $8.20 \mathrm{E}-07$ \\
\hline NM_002135 & NR4A1 & 5.0 & DOWN & $6.03 \mathrm{E}-11$ \\
\hline NM_006705 & GADD45G & 5.0 & DOWN & $6.91 \mathrm{E}-07$ \\
\hline NM_000167 & GK & 5.0 & DOWN & 2.58E-07 \\
\hline NM_006516 & SLC2A1 & 4.9 & DOWN & $1.29 \mathrm{E}-08$ \\
\hline NM_002047 & GARS & 4.9 & DOWN & 1.80E-09 \\
\hline BC001618 & SLC1A4 & 4.9 & DOWN & 1.13E-10 \\
\hline NM_018259 & TTC17 & 4.9 & DOWN & $1.78 \mathrm{E}-08$ \\
\hline AL137311 & DNER & 4.8 & DOWN & $6.64 \mathrm{E}-05$ \\
\hline NM_016242 & EMCN & 4.8 & DOWN & $1.07 \mathrm{E}-05$ \\
\hline
\end{tabular}




\begin{tabular}{|c|c|c|c|c|}
\hline NM_025001 & MTHFD2L & 4.8 & DOWN & $1.41 \mathrm{E}-06$ \\
\hline NM_023940 & RASL11B & 4.8 & DOWN & $2.49 \mathrm{E}-07$ \\
\hline AF022375 & VEGFA & 4.8 & DOWN & $9.52 \mathrm{E}-09$ \\
\hline NM_005261 & GEM & 4.7 & DOWN & $8.77 \mathrm{E}-07$ \\
\hline AF104032 & SLC7A5 & 4.7 & DOWN & $6.15 \mathrm{E}-08$ \\
\hline AL353933 & SLC22A15 & 4.7 & DOWN & $1.74 \mathrm{E}-06$ \\
\hline X63759 & TNP2 & 4.6 & DOWN & $2.34 \mathrm{E}-05$ \\
\hline NM_006260 & DNAJC3 & 4.6 & DOWN & $9.73 \mathrm{E}-07$ \\
\hline NM_031456 & FBXW10 & 4.6 & DOWN & $2.21 \mathrm{E}-06$ \\
\hline AK056433 & VAC14 & 4.6 & DOWN & $3.09 \mathrm{E}-07$ \\
\hline NM_004933 & $\mathrm{CDH} 15$ & 4.6 & DOWN & $5.06 \mathrm{E}-05$ \\
\hline NM_001818 & AKR1C4 & 4.6 & DOWN & 4.55E-07 \\
\hline NM_004524 & LLGL2 & 4.5 & DOWN & $2.49 \mathrm{E}-07$ \\
\hline NM_020184 & CNNM4 & 4.5 & DOWN & $1.24 \mathrm{E}-08$ \\
\hline D31887 & SLC39A14 & 4.4 & DOWN & $8.08 \mathrm{E}-09$ \\
\hline BC012337 & HKDC1 & 4.4 & DOWN & $2.49 \mathrm{E}-07$ \\
\hline NM_022359 & PDE4DIP & 4.4 & DOWN & $6.50 \mathrm{E}-05$ \\
\hline NM_003900 & SQSTM1 & 4.4 & DOWN & $1.85 \mathrm{E}-04$ \\
\hline NM_016306 & DNAJB11 & 4.3 & DOWN & $6.65 \mathrm{E}-07$ \\
\hline NM_018590 & CGAT2_HUMAN & 4.3 & DOWN & 2.46E-07 \\
\hline NM_000169 & GLA & 4.3 & DOWN & $3.47 \mathrm{E}-08$ \\
\hline NM_018112 & TMEM38B & 4.3 & DOWN & $3.30 \mathrm{E}-05$ \\
\hline NM_002943 & RORA & 4.3 & DOWN & $6.47 \mathrm{E}-06$ \\
\hline NM_024111 & CHAC1 & 4.3 & DOWN & $1.58 \mathrm{E}-08$ \\
\hline NM_012410 & SEZ6L2 & 4.3 & DOWN & $1.81 \mathrm{E}-07$ \\
\hline NM_031846 & MAP2 & 4.2 & DOWN & 3.13E-06 \\
\hline AK025379 & PLEKHM1 & 4.2 & DOWN & $3.13 \mathrm{E}-07$ \\
\hline NM_054012 & ASS1 & 4.2 & DOWN & $1.31 \mathrm{E}-04$ \\
\hline NM_004183 & BEST1 & 4.2 & DOWN & $1.01 \mathrm{E}-06$ \\
\hline NM_004911 & PDIA4 & 4.2 & DOWN & $1.04 \mathrm{E}-04$ \\
\hline NM_025195 & TRIB1 & 4.2 & DOWN & $5.75 \mathrm{E}-07$ \\
\hline NM_002640 & SERPINB8 & 4.2 & DOWN & $1.12 \mathrm{E}-08$ \\
\hline NM_002583 & PAWR & 4.1 & DOWN & $4.88 \mathrm{E}-06$ \\
\hline NM_000270 & NP & 4.1 & DOWN & 2.05E-07 \\
\hline NM_052815 & IER3 & 4.1 & DOWN & $5.50 \mathrm{E}-07$ \\
\hline NM_004418 & DUSP2 & 4.1 & DOWN & $1.64 \mathrm{E}-07$ \\
\hline NM_007076 & NP_009007.2 & 4.1 & DOWN & 2.05E-05 \\
\hline NM_032744 & C6orf105 & 4.1 & DOWN & 2.97E-06 \\
\hline NM_016614 & TTRAP & 4.1 & DOWN & 5.95E-07 \\
\hline AF070606 & ATP2B1 & 4.1 & DOWN & $2.39 \mathrm{E}-06$ \\
\hline NM_005504 & BCAT1 & 4.1 & DOWN & $8.11 \mathrm{E}-08$ \\
\hline NM_006769 & LMO4 & 4.0 & DOWN & $2.83 \mathrm{E}-08$ \\
\hline NM_017793 & RPP25 & 4.0 & DOWN & 1.19E-06 \\
\hline NM_032883 & GCX1_HUMAN & 4.0 & DOWN & $9.26 \mathrm{E}-06$ \\
\hline NM_032413 & C15orf48 & 4.0 & DOWN & $3.38 \mathrm{E}-05$ \\
\hline AC004010 & AMIGO2 & 4.0 & DOWN & $2.90 \mathrm{E}-05$ \\
\hline NM_004010 & $\mathrm{DMD}$ & 4.0 & DOWN & 4.45E-05 \\
\hline BC015687 & FLCN & 4.0 & DOWN & $1.69 \mathrm{E}-05$ \\
\hline NM_006670 & TPBG & 4.0 & DOWN & $5.60 \mathrm{E}-06$ \\
\hline NM_002359 & MAFG & 3.9 & DOWN & $9.42 \mathrm{E}-06$ \\
\hline AK022459 & CCPG1 & 3.9 & DOWN & $9.27 \mathrm{E}-07$ \\
\hline NM_003299 & HSP90B1 & 3.9 & DOWN & $7.80 \mathrm{E}-04$ \\
\hline NM_002970 & SAT1 & 3.9 & DOWN & $6.65 \mathrm{E}-06$ \\
\hline NM_001859 & SLC31A1 & 3.9 & DOWN & 8.99E-08 \\
\hline NM_004403 & DFNA5 & 3.9 & DOWN & 1.14E-07 \\
\hline NM_004184 & WARS & 3.9 & DOWN & 3.27E-07 \\
\hline NM_012082 & ZFPM2 & 3.8 & DOWN & 4.09E-04 \\
\hline NM_005842 & SPRY2 & 3.8 & DOWN & $3.35 \mathrm{E}-04$ \\
\hline NM_014191 & SCN8A & 3.8 & DOWN & $3.18 \mathrm{E}-07$ \\
\hline NM_001946 & DUSP6 & 3.8 & DOWN & $5.86 \mathrm{E}-06$ \\
\hline AB046853 & CDK5RAP2 & 3.8 & DOWN & 2.83E-08 \\
\hline NM_006948 & STCH & 3.8 & DOWN & $1.02 \mathrm{E}-05$ \\
\hline
\end{tabular}




\begin{tabular}{|c|c|c|c|c|}
\hline NM_013246 & CLCF1 & 3.8 & DOWN & $8.39 \mathrm{E}-05$ \\
\hline NM_003953 & MPZL1 & 3.8 & DOWN & 4.26E-06 \\
\hline NM_000574 & CD55 & 3.8 & DOWN & 2.12E-07 \\
\hline NM_004047 & ATP6V0B & 3.8 & DOWN & $6.01 \mathrm{E}-07$ \\
\hline AK001865 & ER01LB & 3.8 & DOWN & $6.29 \mathrm{E}-07$ \\
\hline NM_014330 & PPP1R15A & 3.8 & DOWN & 4.68E-07 \\
\hline NM_000120 & EPHX1 & 3.7 & DOWN & 4.06E-10 \\
\hline NM_021928 & SPCS3 & 3.7 & DOWN & $6.93 \mathrm{E}-07$ \\
\hline NM_001912 & CTSL & 3.7 & DOWN & $8.91 \mathrm{E}-06$ \\
\hline NM_000025 & ADRB3 & 3.7 & DOWN & $5.74 \mathrm{E}-08$ \\
\hline AK055094 & MARVELD2 & 3.7 & DOWN & 1.13E-06 \\
\hline NM_002064 & GLRX & 3.7 & DOWN & 2.00E-05 \\
\hline NM_018423 & STYK1 & 3.6 & DOWN & 1.93E-06 \\
\hline NM_002079 & GOT1 & 3.6 & DOWN & $1.07 \mathrm{E}-08$ \\
\hline NM_053040 & C1orf79 & 3.6 & DOWN & $5.56 \mathrm{E}-08$ \\
\hline NM_000311 & PRNP & 3.6 & DOWN & $9.95 \mathrm{E}-07$ \\
\hline NM_024692 & RSNL2 & 3.6 & DOWN & 4.20E-08 \\
\hline AK056477 & GZF1 & 3.6 & DOWN & 1.85E-07 \\
\hline NM_022365 & DNAJC1 & 3.6 & DOWN & $8.28 \mathrm{E}-08$ \\
\hline NM_005596 & NFIB & 3.6 & DOWN & $3.84 \mathrm{E}-05$ \\
\hline AL050090 & MYRIP & 3.5 & DOWN & 1.60E-05 \\
\hline AB051460 & CPEB4 & 3.5 & DOWN & $2.76 \mathrm{E}-07$ \\
\hline NM_001888 & CRYM & 3.5 & DOWN & 1.29E-04 \\
\hline AK024263 & NA & 3.5 & DOWN & $6.20 \mathrm{E}-08$ \\
\hline NM_014900 & COBLL1 & 3.5 & DOWN & 4.09E-05 \\
\hline NM_031471 & URP2_HUMAN & 3.5 & DOWN & 2.13E-06 \\
\hline NM_030751 & SNF1LK & 3.5 & DOWN & 2.61E-05 \\
\hline BC012513 & RND3 & 3.4 & DOWN & 1.85E-06 \\
\hline NM_023016 & ANKRD57 & 3.4 & DOWN & $1.22 \mathrm{E}-06$ \\
\hline AK024261 & $\mathrm{NA}$ & 3.4 & DOWN & 3.13E-06 \\
\hline NM_024324 & CRELD2 & 3.4 & DOWN & 7.36E-05 \\
\hline BC017667 & NAV3 & 3.4 & DOWN & $5.78 \mathrm{E}-04$ \\
\hline NM_024531 & GPR172A & 3.4 & DOWN & 4.83E-07 \\
\hline AF212224 & CLK1 & 3.4 & DOWN & 7.42E-06 \\
\hline NM_033027 & AXUD1 & 3.4 & DOWN & $1.33 \mathrm{E}-05$ \\
\hline NM_057749 & CCNE2 & 3.4 & DOWN & 4.25E-05 \\
\hline NM_017572 & MKNK2 & 3.3 & DOWN & 8.00E-05 \\
\hline NM_001122 & ADFP & 3.3 & DOWN & $5.08 \mathrm{E}-05$ \\
\hline BC015542 & PVR & 3.3 & DOWN & 7.59E-04 \\
\hline NM_005080 & XBP1 & 3.3 & DOWN & 7.44E-06 \\
\hline NM_052960 & RBP7 & 3.3 & DOWN & $1.11 \mathrm{E}-05$ \\
\hline NM_031412 & GABARAPL1 & 3.3 & DOWN & $6.80 \mathrm{E}-05$ \\
\hline NM_031459 & SESN2 & 3.3 & DOWN & $1.30 \mathrm{E}-05$ \\
\hline NM_006546 & IGF2BP1 & 3.2 & DOWN & 3.27E-05 \\
\hline NM_016535 & ZNF581 & 3.2 & DOWN & 2.12E-06 \\
\hline NM_021972 & SPHK1 & 3.2 & DOWN & 4.22E-05 \\
\hline NM_014584 & ER01L & 3.2 & DOWN & 3.56E-07 \\
\hline AB040959 & DFNB31 & 3.2 & DOWN & $3.37 \mathrm{E}-06$ \\
\hline NM_002014 & FKBP4 & 3.2 & DOWN & 2.29E-06 \\
\hline NM_012081 & ELL2 & 3.2 & DOWN & 3.24E-04 \\
\hline NM_005746 & PBEF1 & 3.2 & DOWN & $1.61 \mathrm{E}-05$ \\
\hline NM_001134 & AFP & 3.2 & DOWN & 1.44E-06 \\
\hline NM_004733 & SLC33A1 & 3.2 & DOWN & $2.36 \mathrm{E}-08$ \\
\hline AB007931 & ZUBR1 & 3.2 & DOWN & 4.23E-07 \\
\hline NM_001881 & CREM & 3.2 & DOWN & $3.51 \mathrm{E}-07$ \\
\hline AK001619 & PDE4DIP & 3.1 & DOWN & $1.11 \mathrm{E}-05$ \\
\hline ВC009033 & ASPHD1 & 3.1 & DOWN & $6.47 \mathrm{E}-06$ \\
\hline NM_000876 & IGF2R & 3.1 & DOWN & 3.85E-04 \\
\hline AF131821 & MGLL & 3.1 & DOWN & $9.03 \mathrm{E}-07$ \\
\hline NM_022447 & PAPD5 & 3.1 & DOWN & 2.56E-05 \\
\hline BC013128 & CEBPG & 3.1 & DOWN & $3.13 \mathrm{E}-06$ \\
\hline NM_004673 & ANGPTL1 & 3.1 & DOWN & $3.13 \mathrm{E}-06$ \\
\hline
\end{tabular}




\begin{tabular}{|c|c|c|c|c|}
\hline NM_002910 & RENBP & 3.1 & DOWN & $1.37 \mathrm{E}-05$ \\
\hline AK021789 & Q8NHV5_HUMAN & 3.1 & DOWN & $3.65 \mathrm{E}-06$ \\
\hline NM_012323 & MAFF & 3.1 & DOWN & 7.18E-05 \\
\hline NM_005524 & HES1 & 3.1 & DOWN & $3.81 \mathrm{E}-07$ \\
\hline NM_005279 & GPR1 & 3.1 & DOWN & $5.18 \mathrm{E}-08$ \\
\hline NM_032711 & MAFG & 3.1 & DOWN & $2.79 \mathrm{E}-05$ \\
\hline NM_014674 & EDEM1 & 3.1 & DOWN & $1.14 \mathrm{E}-07$ \\
\hline NM_022751 & FAM59A & 3.1 & DOWN & $2.56 \mathrm{E}-07$ \\
\hline BC011715 & Q8WTY6_HUMAN & 3.1 & DOWN & $1.33 \mathrm{E}-05$ \\
\hline NM_005527 & HSPA1L & 3.1 & DOWN & 2.61E-06 \\
\hline NM_001675 & ATF4 & 3.1 & DOWN & $3.71 \mathrm{E}-07$ \\
\hline AB051541 & KIAA1754 & 3.0 & DOWN & 1.03E-04 \\
\hline NM_018015 & CXorf57 & 3.0 & DOWN & $8.09 \mathrm{E}-06$ \\
\hline NM_002041 & GABPAP & 3.0 & DOWN & $2.58 \mathrm{E}-07$ \\
\hline NM_021938 & BRUNOL5 & 3.0 & DOWN & $1.07 \mathrm{E}-04$ \\
\hline NM_003851 & CREG1 & 3.0 & DOWN & 3.83E-05 \\
\hline BC016024 & FOXA3 & 3.0 & DOWN & $4.69 \mathrm{E}-04$ \\
\hline AK056926 & SLC31A1 & 3.0 & DOWN & $7.98 \mathrm{E}-06$ \\
\hline NM_033280 & SEC11C & 3.0 & DOWN & $5.35 \mathrm{E}-07$ \\
\hline NM_003254 & TIMP1 & 3.0 & DOWN & $1.80 \mathrm{E}-04$ \\
\hline NM_005875 & EIF1B & 3.0 & DOWN & 3.84E-05 \\
\hline BC017650 & C1orf201 & 3.0 & DOWN & $1.30 \mathrm{E}-05$ \\
\hline NM_002849 & PTPRR & 3.0 & DOWN & 4.53E-07 \\
\hline NM_002182 & IL1RAP & 3.0 & DOWN & $1.11 \mathrm{E}-05$ \\
\hline AL136628 & C4orf16 & 3.0 & DOWN & $1.41 \mathrm{E}-06$ \\
\hline NM_003732 & EIF4EBP3 & 3.0 & DOWN & $5.66 \mathrm{E}-06$ \\
\hline NM_000079 & CHRNA1 & 3.0 & DOWN & $9.38 \mathrm{E}-07$ \\
\hline NM_016069 & TIM16_HUMAN & 3.0 & DOWN & $9.18 \mathrm{E}-09$ \\
\hline AJ301564 & C8orf13 & 2.9 & DOWN & 3.34E-06 \\
\hline NM_018421 & TBC1D2 & 2.9 & DOWN & 4.74E-04 \\
\hline NM_013376 & SERTAD1 & 2.9 & DOWN & $5.89 \mathrm{E}-07$ \\
\hline NM_005729 & PPIF & 2.9 & DOWN & $1.04 \mathrm{E}-06$ \\
\hline NM_024610 & HSPBAP1 & 2.9 & DOWN & $1.38 \mathrm{E}-05$ \\
\hline NM_025084 & Q6P168_HUMAN & 2.9 & DOWN & 2.13E-04 \\
\hline AK023769 & C17orf27 & 2.9 & DOWN & $1.20 \mathrm{E}-05$ \\
\hline NM_031232 & APBA2BP & 2.9 & DOWN & $7.25 \mathrm{E}-07$ \\
\hline NM_014710 & GPRASP1 & 2.9 & DOWN & 2.03E-05 \\
\hline NM_005088 & CXYorf3 & 2.9 & DOWN & 8.91E-08 \\
\hline NM_004343 & CALR & 2.9 & DOWN & $4.50 \mathrm{E}-05$ \\
\hline NM_016262 & TUBE1 & 2.9 & DOWN & 2.02E-06 \\
\hline NM_002631 & PGD & 2.9 & DOWN & $1.67 \mathrm{E}-06$ \\
\hline NM_000882 & IL12A & 2.9 & DOWN & $1.99 \mathrm{E}-05$ \\
\hline AJ277587 & SPIRE1 & 2.8 & DOWN & $8.10 \mathrm{E}-05$ \\
\hline NM_022468 & MMP25 & 2.8 & DOWN & $7.53 \mathrm{E}-06$ \\
\hline NM_016522 & NTRI_HUMAN & 2.8 & DOWN & $1.36 \mathrm{E}-07$ \\
\hline NM_021102 & SPINT2 & 2.8 & DOWN & $8.89 \mathrm{E}-04$ \\
\hline AK024858 & LEMD2 & 2.8 & DOWN & $1.91 \mathrm{E}-07$ \\
\hline NM_012385 & NUPR1_HUMAN & 2.8 & DOWN & $5.68 \mathrm{E}-06$ \\
\hline NM_024506 & GLB1L & 2.8 & DOWN & $8.90 \mathrm{E}-05$ \\
\hline NM_000637 & GSR & 2.8 & DOWN & 5.97E-05 \\
\hline AK024597 & TRIM25 & 2.8 & DOWN & $1.10 \mathrm{E}-05$ \\
\hline AK055618 & C18orf19 & 2.8 & DOWN & $8.36 \mathrm{E}-06$ \\
\hline NM_018357 & LARP6 & 2.8 & DOWN & $6.64 \mathrm{E}-06$ \\
\hline NM_002641 & PIGA & 2.8 & DOWN & 2.02E-06 \\
\hline AL080118 & CN109_HUMAN & 2.8 & DOWN & 2.02E-06 \\
\hline NM_005628 & SLC1A5 & 2.8 & DOWN & $6.89 \mathrm{E}-04$ \\
\hline NM_000600 & IL6 & 2.8 & DOWN & 2.91E-05 \\
\hline AK025983 & MGLL & 2.8 & DOWN & $1.78 \mathrm{E}-04$ \\
\hline NM_002928 & RGS16 & 2.8 & DOWN & $1.83 \mathrm{E}-05$ \\
\hline NM_032315 & 653698 & 2.8 & DOWN & $1.62 \mathrm{E}-04$ \\
\hline NM_004281 & BAG3 & 2.8 & DOWN & 8.02E-05 \\
\hline NM_001628 & AKR1B1 & 2.8 & DOWN & $6.83 \mathrm{E}-05$ \\
\hline
\end{tabular}




\begin{tabular}{|c|c|c|c|c|}
\hline NM_015364 & LY96 & 2.8 & DOWN & $1.31 \mathrm{E}-04$ \\
\hline NM_005727 & TSPAN1 & 2.8 & DOWN & $1.30 \mathrm{E}-06$ \\
\hline NM_003670 & BHLHB2 & 2.8 & DOWN & $1.85 \mathrm{E}-06$ \\
\hline AL122070 & Q6ZUG4_HUMAN & 2.8 & DOWN & $7.12 \mathrm{E}-06$ \\
\hline NM_001553 & IGFBP7 & 2.7 & DOWN & $2.47 \mathrm{E}-04$ \\
\hline NM_001746 & CANX & 2.7 & DOWN & 4.55E-07 \\
\hline NM_002090 & CXCL3 & 2.7 & DOWN & $2.25 \mathrm{E}-05$ \\
\hline BC015711 & SPSB1 & 2.7 & DOWN & 3.38E-07 \\
\hline AL389943 & OSBPL10 & 2.7 & DOWN & $4.88 \mathrm{E}-05$ \\
\hline NM_021732 & AVPI1 & 2.7 & DOWN & 3.45E-05 \\
\hline BC014523 & Q7Z6M3_HUMAN & 2.7 & DOWN & $8.25 \mathrm{E}-05$ \\
\hline AB024574 & GTPBP2 & 2.7 & DOWN & $1.80 \mathrm{E}-05$ \\
\hline NM_001695 & ATP6V1C1 & 2.7 & DOWN & $3.09 \mathrm{E}-06$ \\
\hline AF121255 & EIF2C2 & 2.7 & DOWN & $4.50 \mathrm{E}-05$ \\
\hline NM_016947 & G8_HUMAN & 2.7 & DOWN & 1.15E-06 \\
\hline NM_000942 & PPIB & 2.7 & DOWN & $2.73 \mathrm{E}-04$ \\
\hline NM_032873 & STS1_HUMAN & 2.7 & DOWN & $5.66 \mathrm{E}-05$ \\
\hline NM_002022 & FMO4 & 2.7 & DOWN & 2.04E-07 \\
\hline NM_002659 & PLAUR & 2.7 & DOWN & $5.49 \mathrm{E}-05$ \\
\hline AK056836 & C22:IL17RA & 2.7 & DOWN & $6.28 \mathrm{E}-06$ \\
\hline NM_020645 & NRIP3 & 2.7 & DOWN & $1.02 \mathrm{E}-05$ \\
\hline NM_025000 & C2orf37 & 2.7 & DOWN & 7.16E-06 \\
\hline NM_006509 & RELB & 2.7 & DOWN & $6.33 \mathrm{E}-04$ \\
\hline NM_024123 & LY6G6E & 2.6 & DOWN & $9.09 \mathrm{E}-07$ \\
\hline NM_032258 & TBCD3_HUMAN & 2.6 & DOWN & $1.03 E-04$ \\
\hline NM_018234 & STEAP3 & 2.6 & DOWN & $5.17 \mathrm{E}-05$ \\
\hline NM_012296 & GAB2 & 2.6 & DOWN & $2.26 \mathrm{E}-04$ \\
\hline NM_032325 & NP_115701.2 & 2.6 & DOWN & $1.62 \mathrm{E}-06$ \\
\hline BC011763 & TRIM4 & 2.6 & DOWN & $7.98 \mathrm{E}-06$ \\
\hline BC017253 & U31 & 2.6 & DOWN & 1.27E-04 \\
\hline NM_021106 & RGS3 & 2.6 & DOWN & 2.05E-07 \\
\hline AB033112 & BRPF3 & 2.6 & DOWN & $7.35 \mathrm{E}-07$ \\
\hline AK026142 & GATAD1 & 2.6 & DOWN & $6.79 \mathrm{E}-04$ \\
\hline NM_001550 & IFRD1 & 2.6 & DOWN & 1.10E-06 \\
\hline NM_006043 & HS3ST2 & 2.6 & DOWN & 8.05E-05 \\
\hline BC013372 & DEDD2 & 2.6 & DOWN & $1.45 \mathrm{E}-05$ \\
\hline AK024941 & DNAJC3 & 2.6 & DOWN & $1.51 \mathrm{E}-05$ \\
\hline NM_006663 & PPP1R13L & 2.6 & DOWN & 4.69E-05 \\
\hline NM_001728 & BSG & 2.6 & DOWN & $3.01 \mathrm{E}-05$ \\
\hline NM_017784 & OSBPL10 & 2.6 & DOWN & $3.92 \mathrm{E}-06$ \\
\hline AB033029 & JGI:USP31 & 2.6 & DOWN & 8.95E-06 \\
\hline BC010616 & WBP2 & 2.6 & DOWN & 4.81E-06 \\
\hline AL110126 & NFIB & 2.6 & DOWN & $6.94 \mathrm{E}-04$ \\
\hline BC010990 & ZNF598 & 2.6 & DOWN & $2.68 \mathrm{E}-05$ \\
\hline AB051479 & VEPH1 & 2.6 & DOWN & $3.68 \mathrm{E}-06$ \\
\hline NM_052868 & IGSF8 & 2.6 & DOWN & 4.61E-05 \\
\hline NM_014167 & CCDC59 & 2.6 & DOWN & 1.14E-05 \\
\hline NM_013417 & IARS & 2.6 & DOWN & $8.63 \mathrm{E}-05$ \\
\hline AK025100 & SNTB1 & 2.6 & DOWN & $5.93 \mathrm{E}-04$ \\
\hline NM_003904 & ZNF259 & 2.6 & DOWN & $3.63 \mathrm{E}-06$ \\
\hline NM_001751 & CARS & 2.6 & DOWN & $1.66 \mathrm{E}-05$ \\
\hline AK024653 & BCM:bcm2075 & 2.6 & DOWN & $5.56 \mathrm{E}-04$ \\
\hline NM_004272 & HOMER1 & 2.6 & DOWN & $1.46 \mathrm{E}-05$ \\
\hline NM_000849 & GSTM3 & 2.5 & DOWN & 8.99E-06 \\
\hline NM_015513 & CRELD1 & 2.5 & DOWN & $8.00 \mathrm{E}-05$ \\
\hline NM_006756 & TCEA1_HUMAN & 2.5 & DOWN & 4.61E-06 \\
\hline NM_016594 & FKBP11 & 2.5 & DOWN & $5.32 \mathrm{E}-05$ \\
\hline AB046769 & Q9H0M3_HUMAN & 2.5 & DOWN & $1.54 \mathrm{E}-05$ \\
\hline NM_000201 & ICAM1 & 2.5 & DOWN & $2.17 \mathrm{E}-04$ \\
\hline NM_001516 & GTF2H3 & 2.5 & DOWN & $3.63 \mathrm{E}-06$ \\
\hline NM_000904 & NQO2 & 2.5 & DOWN & $1.85 \mathrm{E}-07$ \\
\hline BC014527 & FBXO27 & 2.5 & DOWN & $1.52 \mathrm{E}-05$ \\
\hline
\end{tabular}




\begin{tabular}{|c|c|c|c|c|}
\hline NM_004337 & OSGIN2 & 2.5 & DOWN & $1.62 \mathrm{E}-06$ \\
\hline NM_003414 & ZNF267 & 2.5 & DOWN & $1.11 \mathrm{E}-04$ \\
\hline NM_004990 & MARS & 2.5 & DOWN & 7.96E-05 \\
\hline NM_018556 & SIRPG & 2.5 & DOWN & $9.35 \mathrm{E}-07$ \\
\hline AB058702 & POU2F1 & 2.5 & DOWN & 2.22E-05 \\
\hline BC009558 & TMEM58 & 2.5 & DOWN & $2.58 \mathrm{E}-07$ \\
\hline NM_006287 & TFPI & 2.5 & DOWN & $3.64 \mathrm{E}-04$ \\
\hline AK055484 & STXBP5 & 2.5 & DOWN & $2.99 \mathrm{E}-05$ \\
\hline NM_002314 & LIMK1 & 2.5 & DOWN & $1.06 \mathrm{E}-06$ \\
\hline BC013592 & DDIT4L & 2.5 & DOWN & 8.63E-04 \\
\hline NM_000676 & ADORA2B & 2.5 & DOWN & $8.49 \mathrm{E}-06$ \\
\hline NM_003367 & USF2 & 2.5 & DOWN & 8.17E-05 \\
\hline BC016556 & TMED5 & 2.5 & DOWN & 2.32E-05 \\
\hline AF000560 & ZNF787 & 2.4 & DOWN & $3.76 \mathrm{E}-06$ \\
\hline NM_006513 & SARS & 2.4 & DOWN & 4.03E-06 \\
\hline NM_002342 & LTBR & 2.4 & DOWN & $1.39 \mathrm{E}-04$ \\
\hline NM_022780 & RMND5A & 2.4 & DOWN & 3.17E-06 \\
\hline NM_012124 & CHORDC1 & 2.4 & DOWN & $6.35 \mathrm{E}-07$ \\
\hline BC010112 & HSPD1 & 2.4 & DOWN & $1.45 \mathrm{E}-05$ \\
\hline AF161383 & C6orf129 & 2.4 & DOWN & $8.63 \mathrm{E}-05$ \\
\hline NM_019044 & CCDC93 & 2.4 & DOWN & $7.98 \mathrm{E}-06$ \\
\hline NM_003580 & NSMAF & 2.4 & DOWN & 8.99E-05 \\
\hline NM_002561 & P2RX5 & 2.4 & DOWN & $1.63 \mathrm{E}-04$ \\
\hline AL117608 & FGFR1OP2 & 2.4 & DOWN & $1.61 \mathrm{E}-05$ \\
\hline NM_023076 & C16orf28 & 2.4 & DOWN & $3.41 \mathrm{E}-05$ \\
\hline NM_003764 & STX11 & 2.4 & DOWN & $1.93 \mathrm{E}-05$ \\
\hline NM_019037 & EXOSC4 & 2.4 & DOWN & $1.67 \mathrm{E}-05$ \\
\hline NM_003576 & STK24 & 2.4 & DOWN & $1.73 \mathrm{E}-05$ \\
\hline AY044164 & ALPK1 & 2.4 & DOWN & $6.56 \mathrm{E}-06$ \\
\hline NM_004987 & LIMS1 & 2.4 & DOWN & 3.97E-04 \\
\hline NM_003680 & YARS & 2.4 & DOWN & 3.13E-06 \\
\hline NM_032717 & NP_116106.2 & 2.4 & DOWN & 2.64E-06 \\
\hline NM_000558 & HBA_HUMAN & 2.4 & DOWN & $5.06 \mathrm{E}-05$ \\
\hline NM_024525 & TTC13 & 2.4 & DOWN & $7.60 \mathrm{E}-05$ \\
\hline NM_014278 & HSPA4L & 2.4 & DOWN & $2.13 \mathrm{E}-04$ \\
\hline NM_003875 & GMPS & 2.4 & DOWN & 2.69E-04 \\
\hline NM_012463 & ATP6V0A2 & 2.4 & DOWN & $1.32 \mathrm{E}-04$ \\
\hline NM_002268 & KPNA4 & 2.4 & DOWN & $2.64 \mathrm{E}-06$ \\
\hline NM_032384 & Q9H5Q3_HUMAN & 2.4 & DOWN & $1.61 \mathrm{E}-05$ \\
\hline NM_022448 & Q96E37_HUMAN & 2.4 & DOWN & 2.22E-06 \\
\hline AK055774 & PAQR3 & 2.4 & DOWN & 2.67E-04 \\
\hline NM_006558 & KHDRBS3 & 2.4 & DOWN & $6.45 \mathrm{E}-05$ \\
\hline AF121858 & SNX8 & 2.4 & DOWN & $6.44 \mathrm{E}-06$ \\
\hline NM_000433 & NCF2 & 2.4 & DOWN & $2.76 \mathrm{E}-04$ \\
\hline NM_002056 & GFPT1 & 2.4 & DOWN & $9.07 \mathrm{E}-05$ \\
\hline NM_006755 & TALD01 & 2.4 & DOWN & $4.82 \mathrm{E}-04$ \\
\hline NM_005283 & XCR1 & 2.4 & DOWN & $1.07 \mathrm{E}-04$ \\
\hline BC007429 & STX3 & 2.4 & DOWN & $2.15 \mathrm{E}-05$ \\
\hline U82319 & C6orf68 & 2.3 & DOWN & $8.00 \mathrm{E}-05$ \\
\hline NM_002105 & H2AFX & 2.3 & DOWN & $2.12 \mathrm{E}-04$ \\
\hline NM_022117 & TSPYL2 & 2.3 & DOWN & $9.52 \mathrm{E}-05$ \\
\hline AK025651 & C1orf121 & 2.3 & DOWN & $5.92 \mathrm{E}-04$ \\
\hline NM_004623 & TTC4 & 2.3 & DOWN & $6.40 \mathrm{E}-06$ \\
\hline NM_021194 & SLC30A1 & 2.3 & DOWN & 4.44E-04 \\
\hline NM_004447 & EPS8 & 2.3 & DOWN & $1.31 \mathrm{E}-04$ \\
\hline BE884686 & LTB4DH & 2.3 & DOWN & 1.17E-05 \\
\hline BC000320 & SH3BP1 & 2.3 & DOWN & $5.79 \mathrm{E}-05$ \\
\hline NM_018440 & PAG1 & 2.3 & DOWN & $2.58 \mathrm{E}-05$ \\
\hline AF308297 & PPP1R14C & 2.3 & DOWN & $1.75 \mathrm{E}-04$ \\
\hline NM_005426 & TP53BP2 & 2.3 & DOWN & 4.77E-06 \\
\hline NM_002019 & FLT1 & 2.3 & DOWN & 2.12E-05 \\
\hline AK025306 & CLK1 & 2.3 & DOWN & $1.24 \mathrm{E}-04$ \\
\hline
\end{tabular}




\begin{tabular}{|c|c|c|c|c|}
\hline AL137438 & EXOC6 & 2.3 & DOWN & 5.97E-05 \\
\hline NM_018361 & AGPAT5 & 2.3 & DOWN & $1.46 \mathrm{E}-05$ \\
\hline NM_000713 & BLVRB & 2.3 & DOWN & 7.11E-04 \\
\hline AL050005 & EIF1 & 2.3 & DOWN & $8.58 \mathrm{E}-06$ \\
\hline NM_003806 & HRK & 2.3 & DOWN & $7.70 \mathrm{E}-05$ \\
\hline NM_006931 & SLC2A3 & 2.3 & DOWN & $3.40 \mathrm{E}-06$ \\
\hline NM_014778 & NUPL1 & 2.3 & DOWN & $6.25 \mathrm{E}-04$ \\
\hline NM_032340 & C6orf125 & 2.3 & DOWN & $1.88 \mathrm{E}-06$ \\
\hline NM_007245 & ATXN2L & 2.3 & DOWN & $5.93 \mathrm{E}-04$ \\
\hline NM_004613 & TGM2 & 2.3 & DOWN & $5.67 \mathrm{E}-04$ \\
\hline AK025661 & LIMS1 & 2.3 & DOWN & $1.37 \mathrm{E}-05$ \\
\hline BC009493 & DOLPP1 & 2.3 & DOWN & 4.03E-06 \\
\hline NM_006693 & CPSF4 & 2.3 & DOWN & $3.98 \mathrm{E}-05$ \\
\hline NM_032712 & C19orf48 & 2.3 & DOWN & $6.04 \mathrm{E}-04$ \\
\hline NM_024303 & ZSCAN5 & 2.3 & DOWN & 8.54E-04 \\
\hline AF395440 & Q96RF1_HUMAN & 2.3 & DOWN & $1.01 \mathrm{E}-04$ \\
\hline NM_005887 & DLEU1 & 2.3 & DOWN & $1.27 \mathrm{E}-05$ \\
\hline NM_002486 & NCBP1 & 2.3 & DOWN & $3.70 \mathrm{E}-04$ \\
\hline NM_017983 & WIPI1 & 2.3 & DOWN & $2.79 \mathrm{E}-05$ \\
\hline NM_000935 & PLOD2 & 2.3 & DOWN & $1.13 \mathrm{E}-04$ \\
\hline NM_001539 & DNAJA1 & 2.3 & DOWN & $7.98 \mathrm{E}-06$ \\
\hline BC017085 & SERINC2 & 2.3 & DOWN & $8.32 \mathrm{E}-05$ \\
\hline AK023635 & BCM:bcm2077 & 2.3 & DOWN & 3.35E-04 \\
\hline NM_016274 & PLEKHO1 & 2.3 & DOWN & $2.20 \mathrm{E}-04$ \\
\hline NM_004824 & CDYL & 2.3 & DOWN & $6.20 \mathrm{E}-05$ \\
\hline NM_024640 & YRDC & 2.3 & DOWN & 2.24E-05 \\
\hline NM_003033 & ST3GAL1 & 2.3 & DOWN & $5.77 \mathrm{E}-04$ \\
\hline NM_014068 & PSORS1C1 & 2.3 & DOWN & $5.96 \mathrm{E}-05$ \\
\hline NM_017909 & C6orf96 & 2.3 & DOWN & $8.82 E-05$ \\
\hline NM_024052 & C17orf39 & 2.2 & DOWN & $9.01 \mathrm{E}-06$ \\
\hline NM_006186 & NR4A2 & 2.2 & DOWN & 1.77E-05 \\
\hline NM_005689 & ABCB6 & 2.2 & DOWN & $1.47 \mathrm{E}-04$ \\
\hline NM_003183 & ADAM17 & 2.2 & DOWN & $1.07 \mathrm{E}-05$ \\
\hline NM_002448 & MSX1 & 2.2 & DOWN & 7.96E-05 \\
\hline NM_020122 & KCMF1 & 2.2 & DOWN & $3.18 \mathrm{E}-06$ \\
\hline NM_014851 & KLHL21 & 2.2 & DOWN & $3.10 \mathrm{E}-05$ \\
\hline NM_003244 & TGIF & 2.2 & DOWN & $2.73 \mathrm{E}-04$ \\
\hline AK055902 & KIAA1324L & 2.2 & DOWN & 2.00E-04 \\
\hline AK026295 & SERINC5 & 2.2 & DOWN & 2.54E-05 \\
\hline NM_000916 & OXTR & 2.2 & DOWN & $5.16 \mathrm{E}-04$ \\
\hline NM_002467 & MYC & 2.2 & DOWN & $6.03 E-04$ \\
\hline D26067 & TMEM41B & 2.2 & DOWN & $1.33 \mathrm{E}-05$ \\
\hline NM_023935 & CT116_HUMAN & 2.2 & DOWN & 2.03E-04 \\
\hline NM_016545 & IER5 & 2.2 & DOWN & $6.26 \mathrm{E}-05$ \\
\hline NM_032270 & LRRC8C & 2.2 & DOWN & $1.21 \mathrm{E}-04$ \\
\hline NM_004414 & DSCR1 & 2.2 & DOWN & $1.25 \mathrm{E}-04$ \\
\hline AL050151 & NA & 2.2 & DOWN & 4.73E-05 \\
\hline NM_021013 & KRT34 & 2.2 & DOWN & 7.92E-05 \\
\hline AB023142 & CORO2B & 2.2 & DOWN & $6.60 \mathrm{E}-04$ \\
\hline NM_018162 & NAV2 & 2.2 & DOWN & $6.62 \mathrm{E}-04$ \\
\hline NM_000638 & VTN & 2.2 & DOWN & 2.14E-04 \\
\hline NM_004584 & RAD9A & 2.2 & DOWN & 4.67E-04 \\
\hline NM_004087 & DLG1 & 2.2 & DOWN & $8.62 \mathrm{E}-04$ \\
\hline NM_018214 & LRRC1 & 2.2 & DOWN & 8.92E-06 \\
\hline NM_018840 & CT024_HUMAN & 2.2 & DOWN & 2.91E-05 \\
\hline NM_016639 & TNFRSF12A & 2.2 & DOWN & $2.30 \mathrm{E}-05$ \\
\hline NM_002157 & HSPE1 & 2.2 & DOWN & $1.52 \mathrm{E}-05$ \\
\hline NM_006410 & HTATIP2 & 2.2 & DOWN & $8.71 \mathrm{E}-04$ \\
\hline NM_005415 & SLC20A1 & 2.2 & DOWN & 4.07E-05 \\
\hline NM_002846 & PTPRN & 2.2 & DOWN & $8.88 \mathrm{E}-04$ \\
\hline AK022745 & NA & 2.2 & DOWN & $3.47 \mathrm{E}-05$ \\
\hline NM_014951 & ZNF365 & 2.2 & DOWN & $8.00 \mathrm{E}-05$ \\
\hline
\end{tabular}




\begin{tabular}{|c|c|c|c|c|}
\hline NM_016034 & MRPS2 & 2.2 & DOWN & $6.87 \mathrm{E}-05$ \\
\hline BC007644 & YIF1B & 2.2 & DOWN & $7.59 \mathrm{E}-04$ \\
\hline AB007923 & PDE4DIP & 2.2 & DOWN & $3.80 \mathrm{E}-05$ \\
\hline U92285 & GABRE & 2.2 & DOWN & 2.67E-04 \\
\hline NM_001064 & TKT & 2.2 & DOWN & 7.57E-05 \\
\hline NM_004405 & DLX2 & 2.2 & DOWN & 1.07E-04 \\
\hline NM_006278 & ST3GAL4 & 2.2 & DOWN & $1.42 \mathrm{E}-06$ \\
\hline NM_001909 & CTSD & 2.2 & DOWN & $8.85 \mathrm{E}-06$ \\
\hline NM_007314 & ABL2 & 2.2 & DOWN & 2.03E-05 \\
\hline AK026436 & ARRDC4 & 2.2 & DOWN & $5.14 \mathrm{E}-04$ \\
\hline NM_005228 & EGFR & 2.2 & DOWN & $8.99 \mathrm{E}-05$ \\
\hline AB032972 & NA & 2.2 & DOWN & 2.77E-04 \\
\hline NM_005627 & SGK & 2.2 & DOWN & $5.98 \mathrm{E}-04$ \\
\hline AL137488 & C12orf29 & 2.1 & DOWN & $3.56 \mathrm{E}-04$ \\
\hline NM_005176 & ATP5G2 & 2.1 & DOWN & $9.21 \mathrm{E}-04$ \\
\hline BC008580 & GDNF & 2.1 & DOWN & $5.05 \mathrm{E}-05$ \\
\hline Z46376 & HK2 & 2.1 & DOWN & $2.42 \mathrm{E}-04$ \\
\hline NM_005194 & CEBPB & 2.1 & DOWN & $1.61 \mathrm{E}-05$ \\
\hline NM_022102 & NP_001026883.1 & 2.1 & DOWN & 3.60E-04 \\
\hline NM_024945 & RMl1 & 2.1 & DOWN & $1.61 \mathrm{E}-05$ \\
\hline AK026217 & TTC17 & 2.1 & DOWN & $1.83 \mathrm{E}-05$ \\
\hline NM_016227 & C1orf9 & 2.1 & DOWN & 7.05E-04 \\
\hline NM_030759 & NRBF2 & 2.1 & DOWN & $1.32 \mathrm{E}-05$ \\
\hline AK057591 & DGKE & 2.1 & DOWN & $7.21 \mathrm{E}-05$ \\
\hline AL136770 & CLDN12 & 2.1 & DOWN & $2.28 \mathrm{E}-04$ \\
\hline NM_014423 & AFF4 & 2.1 & DOWN & $8.29 \mathrm{E}-05$ \\
\hline NM_032331 & ECE2 & 2.1 & DOWN & 5.97E-04 \\
\hline NM_003155 & STC1 & 2.1 & DOWN & $1.64 \mathrm{E}-04$ \\
\hline AB011164 & NP_001005751.1 & 2.1 & DOWN & $1.04 \mathrm{E}-06$ \\
\hline NM_006042 & HS3ST3A1 & 2.1 & DOWN & $2.39 \mathrm{E}-04$ \\
\hline NM_002507 & NGFR & 2.1 & DOWN & $8.02 \mathrm{E}-05$ \\
\hline NM_000735 & CGA & 2.1 & DOWN & $4.14 \mathrm{E}-04$ \\
\hline NM_004388 & CTBS & 2.1 & DOWN & $1.31 \mathrm{E}-04$ \\
\hline NM_004563 & PCK2 & 2.1 & DOWN & $9.58 \mathrm{E}-04$ \\
\hline BC017117 & CREM & 2.1 & DOWN & $7.98 \mathrm{E}-06$ \\
\hline NM_024039 & MIS12 & 2.1 & DOWN & $6.19 \mathrm{E}-07$ \\
\hline AK022547 & PARP6 & 2.1 & DOWN & 7.36E-05 \\
\hline NM_021226 & ARHGAP22 & 2.1 & DOWN & $7.82 \mathrm{E}-04$ \\
\hline NM_013326 & C18orf8 & 2.1 & DOWN & 3.33E-04 \\
\hline NM_024937 & LRP12 & 2.1 & DOWN & $3.04 \mathrm{E}-04$ \\
\hline NM_018231 & NP_060701.1 & 2.1 & DOWN & $2.30 \mathrm{E}-05$ \\
\hline NM_001294 & CLPTM1 & 2.1 & DOWN & $2.94 \mathrm{E}-04$ \\
\hline NM_002423 & MMP7 & 2.1 & DOWN & $6.74 \mathrm{E}-04$ \\
\hline NM_000903 & NQ01 & 2.1 & DOWN & 8.93E-05 \\
\hline NM_025128 & MUS81 & 2.1 & DOWN & $8.53 \mathrm{E}-05$ \\
\hline NM_014725 & STARD8 & 2.1 & DOWN & $1.75 \mathrm{E}-04$ \\
\hline NM_005706 & TSSC4 & 2.1 & DOWN & $1.75 \mathrm{E}-04$ \\
\hline BC012469 & YIPF6 & 2.1 & DOWN & $3.59 \mathrm{E}-05$ \\
\hline NM_004717 & DGKI & 2.1 & DOWN & $7.25 \mathrm{E}-04$ \\
\hline NM_012099 & CD3EAP & 2.1 & DOWN & $4.90 \mathrm{E}-06$ \\
\hline AL021327 & RP1-12409.1 & 2.1 & DOWN & $9.02 \mathrm{E}-06$ \\
\hline AF161415 & SELK_HUMAN & 2.1 & DOWN & 7.26E-06 \\
\hline NM_003301 & TRHR & 2.1 & DOWN & $6.08 \mathrm{E}-04$ \\
\hline AF151867 & TICAM2 & 2.1 & DOWN & $1.01 \mathrm{E}-05$ \\
\hline AK023623 & GLDN & 2.1 & DOWN & 4.44E-04 \\
\hline NM_013379 & DPP7 & 2.1 & DOWN & 2.77E-04 \\
\hline NM_005681 & TAF1A & 2.0 & DOWN & $6.39 \mathrm{E}-04$ \\
\hline NM_005336 & HDLBP & 2.0 & DOWN & $9.52 \mathrm{E}-04$ \\
\hline NM_006207 & PDGFRL & 2.0 & DOWN & $8.88 \mathrm{E}-04$ \\
\hline AK055649 & ERICH1 & 2.0 & DOWN & 4.53E-05 \\
\hline NM_019025 & SMOX & 2.0 & DOWN & 4.16E-05 \\
\hline AF062733 & IGSF4B & 2.0 & DOWN & $1.33 \mathrm{E}-04$ \\
\hline
\end{tabular}




\begin{tabular}{|c|c|c|c|c|}
\hline M12679 & HLA-C & 2.0 & DOWN & $2.21 \mathrm{E}-04$ \\
\hline NM_022758 & C6orf106 & 2.0 & DOWN & 8.29E-04 \\
\hline NM_024686 & TTLL7 & 2.0 & DOWN & 3.65E-05 \\
\hline NM_015449 & C1orf43 & 2.0 & DOWN & 4.95E-04 \\
\hline NM_005738 & ARL4A & 2.0 & DOWN & 1.85E-04 \\
\hline BC010612 & Q96FP1_HUMAN & 2.0 & DOWN & 4.73E-04 \\
\hline NM_002249 & KCNN3 & 2.0 & DOWN & 1.03E-04 \\
\hline NM_007355 & HSP90AB1 & 2.0 & DOWN & $1.56 \mathrm{E}-04$ \\
\hline NM_005923 & MAP3K5 & 2.0 & DOWN & $1.73 \mathrm{E}-05$ \\
\hline NM_012111 & AHSA1 & 2.0 & DOWN & $1.48 \mathrm{E}-04$ \\
\hline M75883 & SCP2 & 2.0 & UP & 2.27E-04 \\
\hline NM_057161 & KLHDC3 & 2.0 & UP & $5.54 \mathrm{E}-05$ \\
\hline AK001731 & C12orf23 & 2.0 & UP & $1.51 \mathrm{E}-05$ \\
\hline NM_018007 & FBXO4 & 2.0 & UP & $1.22 \mathrm{E}-04$ \\
\hline AL049949 & C10orf56 & 2.0 & UP & 4.53E-04 \\
\hline AB020689 & TBC1D9 & 2.0 & UP & $2.56 \mathrm{E}-04$ \\
\hline NM_024833 & ZNF671 & 2.0 & UP & $9.53 \mathrm{E}-04$ \\
\hline NM_015559 & SETBP1 & 2.0 & UP & 2.61E-05 \\
\hline AK021851 & NA & 2.0 & UP & 5.77E-04 \\
\hline NM_000235 & LIPA & 2.0 & UP & 4.31E-04 \\
\hline AB007855 & $\mathrm{ZHX3}$ & 2.0 & UP & 8.63E-05 \\
\hline NM_017458 & MVP & 2.0 & UP & $8.86 \mathrm{E}-05$ \\
\hline NM_000947 & PRIM2A & 2.0 & UP & $5.98 \mathrm{E}-05$ \\
\hline AK021807 & LRP11 & 2.0 & UP & 4.34E-04 \\
\hline NM_005327 & $\mathrm{HADH}$ & 2.0 & UP & $4.62 \mathrm{E}-05$ \\
\hline AK056552 & ZNF528 & 2.0 & UP & 3.21E-04 \\
\hline NM_023037 & FRY & 2.0 & UP & 7.01E-05 \\
\hline D25304 & ARHGEF6 & 2.0 & UP & $7.88 \mathrm{E}-04$ \\
\hline NM_000110 & DPYD & 2.0 & UP & $1.39 \mathrm{E}-06$ \\
\hline AB033056 & PPFIBP1 & 2.0 & UP & 7.36E-04 \\
\hline NM_002129 & HMGB2 & 2.0 & UP & $5.41 \mathrm{E}-04$ \\
\hline AK057365 & TLOC1 & 2.1 & UP & 7.63E-05 \\
\hline BC013153 & LOXL4 & 2.1 & UP & $6.22 \mathrm{E}-04$ \\
\hline AK001553 & AK3 & 2.1 & UP & 3.60E-04 \\
\hline NM_018719 & CDCA7L & 2.1 & UP & $3.01 \mathrm{E}-05$ \\
\hline NM_014928 & OTUD4 & 2.1 & UP & 1.27E-04 \\
\hline AK055981 & NA & 2.1 & UP & 2.77E-04 \\
\hline AK058159 & DHRS1 & 2.1 & UP & 3.97E-04 \\
\hline NM_012446 & SSBP2 & 2.1 & UP & 3.96E-05 \\
\hline AB007916 & SLC35E2 & 2.1 & UP & 1.75E-04 \\
\hline NM_014055 & IFT81 & 2.1 & UP & $1.11 \mathrm{E}-04$ \\
\hline NM_018222 & PARVA & 2.1 & UP & 7.67E-04 \\
\hline AL080186 & C6orf111 & 2.1 & UP & $9.00 \mathrm{E}-05$ \\
\hline AK056245 & ZMAT3 & 2.1 & UP & 7.37E-04 \\
\hline AJ420416 & GCNT1 & 2.1 & UP & 3.04E-04 \\
\hline NM_022918 & TMEM135 & 2.1 & UP & 3.09E-04 \\
\hline NM_001635 & AMPH & 2.1 & UP & 3.13E-04 \\
\hline BC014890 & SNAI2 & 2.1 & UP & 5.93E-04 \\
\hline AJ420488 & EEF1A1 & 2.1 & UP & $8.56 \mathrm{E}-05$ \\
\hline D50683 & TGFBR2 & 2.1 & UP & 8.75E-04 \\
\hline NM_006275 & SFRS6 & 2.1 & UP & 1.09E-04 \\
\hline NM_014799 & HEPH & 2.1 & UP & 3.79E-04 \\
\hline AF010236 & SGCD & 2.1 & UP & $5.51 \mathrm{E}-04$ \\
\hline AL049471 & ARID5B & 2.1 & UP & $9.91 \mathrm{E}-06$ \\
\hline AK001478 & RHOU & 2.1 & UP & 4.50E-05 \\
\hline NM_022470 & ZMAT3 & 2.1 & UP & 2.67E-04 \\
\hline D42043 & RFTN1 & 2.1 & UP & $6.48 \mathrm{E}-05$ \\
\hline NM_018486 & HDAC8 & 2.1 & UP & 2.67E-04 \\
\hline AB007960 & SH3GLB1 & 2.1 & UP & $6.82 \mathrm{E}-05$ \\
\hline NM_000093 & COL5A1 & 2.1 & UP & 8.44E-06 \\
\hline AB020671 & MRIP_HUMAN & 2.1 & UP & 7.02E-04 \\
\hline NM_021210 & TRAPPC1 & 2.1 & UP & $1.97 \mathrm{E}-04$ \\
\hline
\end{tabular}




\begin{tabular}{|c|c|c|c|c|}
\hline NM_024582 & FAT4 & 2.1 & UP & 4.90E-05 \\
\hline NM_024062 & VANGL1 & 2.1 & UP & $3.60 \mathrm{E}-04$ \\
\hline NM_004765 & BCL7C & 2.1 & UP & $1.37 \mathrm{E}-04$ \\
\hline NM_000107 & DDB2 & 2.1 & UP & $1.90 \mathrm{E}-05$ \\
\hline NM_018355 & ZNF415 & 2.1 & UP & 7.57E-04 \\
\hline NM_002655 & PLAG1 & 2.1 & UP & $2.42 \mathrm{E}-04$ \\
\hline AL136680 & GBP3 & 2.1 & UP & 8.99E-05 \\
\hline NM_006645 & STARD10 & 2.1 & UP & $1.43 \mathrm{E}-04$ \\
\hline AK026717 & SEPW1 & 2.1 & UP & 4.12E-04 \\
\hline NM_005512 & LRRC32 & 2.1 & UP & 7.81E-04 \\
\hline NM_018649 & H2AFY2 & 2.1 & UP & $1.25 \mathrm{E}-04$ \\
\hline NM_000430 & PAFAH1B1 & 2.1 & UP & $9.67 \mathrm{E}-04$ \\
\hline NM_016201 & AMOTL2 & 2.1 & UP & $1.81 \mathrm{E}-05$ \\
\hline AB040929 & CNTN3 & 2.1 & UP & $3.85 \mathrm{E}-04$ \\
\hline X62048 & WEE1 & 2.2 & UP & $6.91 \mathrm{E}-05$ \\
\hline NM_020215 & C14orf132 & 2.2 & UP & $1.52 \mathrm{E}-05$ \\
\hline AB037775 & KLHL9 & 2.2 & UP & $6.56 \mathrm{E}-05$ \\
\hline NM_006074 & TRIM22 & 2.2 & UP & $3.62 \mathrm{E}-04$ \\
\hline NM_004776 & B4GALT5 & 2.2 & UP & 4.09E-04 \\
\hline AB020710 & EHBP1 & 2.2 & UP & $8.86 \mathrm{E}-05$ \\
\hline NM_025149 & NP_079425.2 & 2.2 & UP & $9.59 \mathrm{E}-04$ \\
\hline NM_019035 & PCDH18 & 2.2 & UP & $2.67 \mathrm{E}-04$ \\
\hline AL117621 & ARF6 & 2.2 & UP & $5.06 \mathrm{E}-04$ \\
\hline NM_001114 & ADCY7 & 2.2 & UP & 4.50E-04 \\
\hline AF131851 & FAM19A5 & 2.2 & UP & 4.67E-06 \\
\hline AY039760 & ATP6V1G3 & 2.2 & UP & $7.30 \mathrm{E}-04$ \\
\hline NM_005766 & FARP1 & 2.2 & UP & 4.46E-04 \\
\hline NM_006111 & ACAA2 & 2.2 & UP & 1.67E-06 \\
\hline NM_014621 & NA & 2.2 & UP & $5.98 \mathrm{E}-04$ \\
\hline NM_001330 & CTF1 & 2.2 & UP & 2.15E-04 \\
\hline AK056473 & FAM33A & 2.2 & UP & $4.89 \mathrm{E}-04$ \\
\hline NM_005637 & SS18 & 2.2 & UP & $9.90 \mathrm{E}-04$ \\
\hline AK057180 & C16orf45 & 2.2 & UP & 1.13E-04 \\
\hline NM_005584 & MAB21L1 & 2.2 & UP & $8.40 \mathrm{E}-04$ \\
\hline NM_022173 & TIA1 & 2.2 & UP & 4.14E-04 \\
\hline AK026666 & NP_001026897.1 & 2.2 & UP & $1.26 \mathrm{E}-04$ \\
\hline BC008377 & EPB41L3 & 2.2 & UP & $1.58 \mathrm{E}-04$ \\
\hline NM_032305 & POLR3GL & 2.2 & UP & $7.35 \mathrm{E}-05$ \\
\hline AK026977 & MYH10 & 2.2 & UP & $2.34 \mathrm{E}-05$ \\
\hline NM_024830 & AYTL2 & 2.2 & UP & $5.33 \mathrm{E}-06$ \\
\hline NM_014362 & $\mathrm{HIBCH}$ & 2.2 & UP & $6.38 \mathrm{E}-06$ \\
\hline NM_005578 & BCM:LPP & 2.2 & UP & 7.91E-04 \\
\hline AB051491 & KIAA1704 & 2.2 & UP & 4.91E-04 \\
\hline АВ037796 & PDCD6IP & 2.2 & UP & $1.34 \mathrm{E}-05$ \\
\hline NM_000077 & CDKN2A & 2.2 & UP & $9.76 \mathrm{E}-04$ \\
\hline NM_014970 & KIFAP3 & 2.2 & UP & 4.74E-06 \\
\hline NM_006226 & PLCL1 & 2.2 & UP & 7.44E-04 \\
\hline AB051515 & TANC1 & 2.2 & UP & $2.21 \mathrm{E}-05$ \\
\hline AL157488 & NA & 2.2 & UP & $6.28 \mathrm{E}-04$ \\
\hline BC013920 & NP_997259.1 & 2.2 & UP & 4.02E-05 \\
\hline NM_005566 & LDHA & 2.2 & UP & $2.81 \mathrm{E}-04$ \\
\hline NM_005573 & LMNB1 & 2.2 & UP & $1.38 \mathrm{E}-05$ \\
\hline AL110152 & CD109 & 2.2 & UP & 7.66E-04 \\
\hline NM_001182 & ALDH7A1 & 2.2 & UP & 3.14E-04 \\
\hline AB014540 & SWP70_HUMAN & 2.2 & UP & $1.82 \mathrm{E}-04$ \\
\hline NM_005891 & АCAT2 & 2.3 & UP & $6.70 \mathrm{E}-04$ \\
\hline NM_014965 & TRAK1 & 2.3 & UP & $4.28 \mathrm{E}-04$ \\
\hline NM_001904 & CTNNB1 & 2.3 & UP & $2.54 \mathrm{E}-05$ \\
\hline NM_005630 & SLCO2A1 & 2.3 & UP & $2.43 \mathrm{E}-04$ \\
\hline BC006428 & CXXC5 & 2.3 & UP & 2.66E-06 \\
\hline AL049331 & NME7 & 2.3 & UP & 3.04E-04 \\
\hline AF161403 & CLEC14A & 2.3 & UP & $6.13 \mathrm{E}-04$ \\
\hline
\end{tabular}




\begin{tabular}{|c|c|c|c|c|}
\hline NM_005566 & LDHA & 2.3 & UP & 1.17E-04 \\
\hline NM_005423 & TFF2 & 2.3 & UP & $8.58 \mathrm{E}-04$ \\
\hline NM_005566 & LDHA & 2.3 & UP & 7.67E-05 \\
\hline AB032261 & $S C D$ & 2.3 & UP & $5.98 \mathrm{E}-04$ \\
\hline AB046781 & UACA & 2.3 & UP & $1.82 \mathrm{E}-06$ \\
\hline NM_007083 & NUDT6 & 2.3 & UP & 1.87E-04 \\
\hline AB014589 & CSTF2T & 2.3 & UP & $7.50 \mathrm{E}-05$ \\
\hline NM_057174 & PEX16 & 2.3 & UP & $2.42 \mathrm{E}-04$ \\
\hline D42047 & GPD1L & 2.3 & UP & $1.00 \mathrm{E}-04$ \\
\hline AK025703 & NP_001001701.1 & 2.3 & UP & $1.23 \mathrm{E}-04$ \\
\hline NM_000846 & GSTA1 & 2.3 & UP & 3.06E-05 \\
\hline AB029018 & PDZRN3 & 2.3 & UP & $3.02 E-05$ \\
\hline NM_003634 & NIPSNAP1 & 2.3 & UP & 7.04E-05 \\
\hline BC013049 & MRRF & 2.3 & UP & $2.32 \mathrm{E}-05$ \\
\hline NM_014867 & KBTBD11 & 2.3 & UP & $7.23 \mathrm{E}-05$ \\
\hline NM_000527 & LDLR & 2.3 & UP & 7.84E-05 \\
\hline NM_014456 & PDCD4 & 2.3 & UP & $9.77 \mathrm{E}-05$ \\
\hline AK027274 & MARCKS & 2.3 & UP & $1.03 E-05$ \\
\hline NM_003278 & CLEC3B & 2.3 & UP & 4.45E-04 \\
\hline AK055553 & C22:RP3-477H23.1 & 2.3 & UP & $3.09 \mathrm{E}-04$ \\
\hline AJ312319 & 40057 & 2.3 & UP & $1.65 \mathrm{E}-06$ \\
\hline NM_006579 & EBP & 2.3 & UP & $3.75 \mathrm{E}-04$ \\
\hline NM_003069 & SMARCA1 & 2.3 & UP & $7.89 \mathrm{E}-06$ \\
\hline NM_000671 & ADH5 & 2.3 & UP & 4.64E-06 \\
\hline NM_018159 & NUDT11 & 2.4 & UP & 4.95E-04 \\
\hline NM_017885 & HCFC1R1 & 2.4 & UP & $8.01 \mathrm{E}-05$ \\
\hline AK057328 & NA & 2.4 & UP & $2.21 \mathrm{E}-04$ \\
\hline NM_018422 & Q7L3Y3_HUMAN & 2.4 & UP & $3.13 \mathrm{E}-05$ \\
\hline NM_012484 & HMMR & 2.4 & UP & $5.56 \mathrm{E}-04$ \\
\hline AL049957 & CD59 & 2.4 & UP & $5.08 \mathrm{E}-05$ \\
\hline AK025759 & NA & 2.4 & UP & $2.74 \mathrm{E}-04$ \\
\hline AK057652 & NA & 2.4 & UP & $5.06 \mathrm{E}-04$ \\
\hline NM_012304 & FBXL7 & 2.4 & UP & $2.42 \mathrm{E}-06$ \\
\hline NM_001548 & IFIT1 & 2.4 & UP & 4.70E-04 \\
\hline NM_014937 & INPP5F & 2.4 & UP & $5.68 \mathrm{E}-06$ \\
\hline NM_006412 & AGPAT2 & 2.4 & UP & $1.54 \mathrm{E}-05$ \\
\hline AF070617 & C5orf21 & 2.4 & UP & $1.61 \mathrm{E}-05$ \\
\hline AK058001 & DENND3 & 2.4 & UP & 4.91E-04 \\
\hline AL137535 & C9orf3 & 2.4 & UP & 1.45E-04 \\
\hline NM_002775 & HTRA1 & 2.4 & UP & $1.75 \mathrm{E}-04$ \\
\hline NM_014244 & ADAMTS2 & 2.4 & UP & $8.27 \mathrm{E}-06$ \\
\hline AL117234 & MCF2 & 2.4 & UP & 2.24E-04 \\
\hline AB028976 & GC:SAMD4A & 2.4 & UP & $2.54 \mathrm{E}-05$ \\
\hline NM_032943 & SYTL2 & 2.4 & UP & 1.44E-04 \\
\hline NM_005864 & EFS & 2.5 & UP & 4.14E-04 \\
\hline AB038653 & NA & 2.5 & UP & $1.13 \mathrm{E}-06$ \\
\hline AK056513 & NP_653327.1 & 2.5 & UP & 7.89E-06 \\
\hline AK055087 & C1orf59 & 2.5 & UP & $1.62 \mathrm{E}-06$ \\
\hline NM_013258 & PYCARD & 2.5 & UP & $1.64 \mathrm{E}-04$ \\
\hline NM_018171 & DP13B_HUMAN & 2.5 & UP & $2.72 \mathrm{E}-04$ \\
\hline NM_005418 & ST5 & 2.5 & UP & $1.82 \mathrm{E}-05$ \\
\hline NM_032601 & MCEE & 2.5 & UP & $4.40 \mathrm{E}-07$ \\
\hline BC000268 & PSMB2 & 2.5 & UP & $8.46 \mathrm{E}-04$ \\
\hline NM_016121 & KCTD3 & 2.5 & UP & $5.69 \mathrm{E}-05$ \\
\hline NM_006810 & PDIA5 & 2.5 & UP & $1.85 \mathrm{E}-05$ \\
\hline AL137938 & PXK & 2.5 & UP & 2.11E-05 \\
\hline NM_052954 & CYYR1 & 2.5 & UP & $3.11 \mathrm{E}-04$ \\
\hline AK055466 & C7orf41 & 2.5 & UP & $5.58 \mathrm{E}-05$ \\
\hline AB011153 & PLCB1 & 2.5 & UP & $1.58 \mathrm{E}-04$ \\
\hline AB033025 & KIAA1199 & 2.5 & UP & $9.60 \mathrm{E}-04$ \\
\hline NM_012157 & FBXL2 & 2.5 & UP & $3.60 \mathrm{E}-04$ \\
\hline AK056791 & MUC16 & 2.5 & UP & $3.65 \mathrm{E}-05$ \\
\hline
\end{tabular}




\begin{tabular}{|c|c|c|c|c|}
\hline NM_005566 & LDHA & 2.5 & UP & $1.15 \mathrm{E}-04$ \\
\hline BC000977 & ALAD & 2.5 & UP & $3.83 E-05$ \\
\hline NM_017671 & C20orf42 & 2.5 & UP & 8.03E-04 \\
\hline NM_003711 & PPAP2A & 2.5 & UP & $9.67 \mathrm{E}-04$ \\
\hline AK023762 & SPTBN1 & 2.6 & UP & 4.21E-06 \\
\hline NM_005566 & LDHA & 2.6 & UP & 2.24E-04 \\
\hline AK026140 & FAM20C & 2.6 & UP & $1.69 \mathrm{E}-04$ \\
\hline AK024677 & ASAHL & 2.6 & UP & $9.76 \mathrm{E}-04$ \\
\hline AK055808 & NP_001070148.1 & 2.6 & UP & $6.14 \mathrm{E}-05$ \\
\hline BC015050 & OIP5 & 2.6 & UP & $9.90 \mathrm{E}-04$ \\
\hline AF380356 & $X G$ & 2.6 & UP & $7.28 \mathrm{E}-04$ \\
\hline NM_000859 & HMGCR & 2.6 & UP & $3.28 \mathrm{E}-04$ \\
\hline NM_005463 & HNRPDL & 2.6 & UP & $1.45 \mathrm{E}-06$ \\
\hline NM_005566 & LDHA & 2.6 & UP & $4.50 \mathrm{E}-05$ \\
\hline NM_003250 & THRA & 2.6 & UP & 2.29E-06 \\
\hline NM_019555 & ARHGEF3 & 2.6 & UP & 8.91E-04 \\
\hline NM_033115 & NP_149106.2 & 2.6 & UP & $8.08 \mathrm{E}-06$ \\
\hline AB033060 & PDCD6 & 2.6 & UP & $2.18 \mathrm{E}-06$ \\
\hline NM_001336 & CTSZ & 2.6 & UP & $8.18 \mathrm{E}-05$ \\
\hline NM_004772 & C5orf13 & 2.6 & UP & 2.44E-05 \\
\hline AB051459 & DJBP_HUMAN & 2.6 & UP & $7.52 \mathrm{E}-06$ \\
\hline NM_000393 & COL5A2 & 2.6 & UP & $3.01 \mathrm{E}-05$ \\
\hline NM_000791 & DHFRL1 & 2.6 & UP & $1.62 \mathrm{E}-05$ \\
\hline AF019226 & NA & 2.6 & UP & 2.85E-05 \\
\hline AK026035 & NA & 2.6 & UP & $6.14 \mathrm{E}-05$ \\
\hline AK021980 & TCF4 & 2.6 & UP & $8.56 \mathrm{E}-04$ \\
\hline AK057477 & HYLS1 & 2.6 & UP & 2.85E-05 \\
\hline AK055341 & DAB1 & 2.7 & UP & $3.62 \mathrm{E}-05$ \\
\hline NM_002874 & RAD23B & 2.7 & UP & $2.27 \mathrm{E}-05$ \\
\hline AK023464 & BCM:bcm899 & 2.7 & UP & $5.72 \mathrm{E}-06$ \\
\hline NM_002609 & PDGFRB & 2.7 & UP & 4.10E-06 \\
\hline AL162068 & NAP1L1 & 2.7 & UP & $1.08 \mathrm{E}-06$ \\
\hline BC000039 & FAM26B & 2.7 & UP & $1.82 \mathrm{E}-06$ \\
\hline NM_021953 & FOXM1 & 2.7 & UP & $1.09 \mathrm{E}-04$ \\
\hline NM_001431 & EPB41L2 & 2.7 & UP & $4.50 \mathrm{E}-05$ \\
\hline NM_000557 & GDF5 & 2.7 & UP & $8.98 \mathrm{E}-05$ \\
\hline NM_002863 & PYGL & 2.7 & UP & $2.51 \mathrm{E}-05$ \\
\hline NM_001387 & DPYSL3 & 2.7 & UP & 4.53E-05 \\
\hline NM_004881 & TP5313 & 2.7 & UP & $5.23 \mathrm{E}-08$ \\
\hline NM_005566 & LDHA & 2.7 & UP & 4.21E-05 \\
\hline BC009566 & TAF9B & 2.7 & UP & 2.61E-05 \\
\hline AK023607 & SASH1 & 2.7 & UP & $1.75 \mathrm{E}-06$ \\
\hline NM_000163 & GHR & 2.7 & UP & 4.05E-04 \\
\hline NM_005308 & GRK5 & 2.8 & UP & $1.74 \mathrm{E}-08$ \\
\hline AK025071 & SPTBN1 & 2.8 & UP & $5.98 \mathrm{E}-07$ \\
\hline NM_014051 & TMEM14A & 2.8 & UP & $1.23 \mathrm{E}-06$ \\
\hline NM_022129 & MAWBP_HUMAN & 2.8 & UP & 4.97E-05 \\
\hline NM_000900 & MGP & 2.8 & UP & 2.87E-05 \\
\hline AK057333 & C11orf66 & 2.8 & UP & $1.66 \mathrm{E}-04$ \\
\hline NM_007066 & PKIG & 2.8 & UP & $6.22 \mathrm{E}-04$ \\
\hline M62895 & ANXA2P3 & 2.8 & UP & 1.26E-04 \\
\hline AJ245539 & GALNT5 & 2.8 & UP & $7.86 \mathrm{E}-07$ \\
\hline NM_000147 & FUCA1 & 2.8 & UP & $1.75 \mathrm{E}-05$ \\
\hline NM_022343 & GAPR1_HUMAN & 2.8 & UP & $2.31 \mathrm{E}-06$ \\
\hline NM_005566 & LDHA & 2.8 & UP & $8.02 \mathrm{E}-06$ \\
\hline NM_024049 & RP11-445H22.3 & 2.8 & UP & $9.33 \mathrm{E}-04$ \\
\hline NM_005572 & LMNA & 2.8 & UP & $1.33 \mathrm{E}-05$ \\
\hline NM_001788 & 39326 & 2.8 & UP & $5.71 \mathrm{E}-06$ \\
\hline NM_014011 & socs5 & 2.8 & UP & 2.27E-07 \\
\hline NM_005032 & PLS3 & 2.9 & UP & 7.42E-06 \\
\hline NM_003022 & SH3BGRL & 2.9 & UP & $6.86 \mathrm{E}-07$ \\
\hline NM_007222 & ZHX1 & 2.9 & UP & $1.79 \mathrm{E}-05$ \\
\hline
\end{tabular}




\begin{tabular}{|c|c|c|c|c|}
\hline NM_014217 & KCNK2 & 2.9 & UP & $1.36 \mathrm{E}-05$ \\
\hline BC013939 & NA & 2.9 & UP & $4.30 \mathrm{E}-05$ \\
\hline NM_003243 & TGFBR3 & 2.9 & UP & $2.01 \mathrm{E}-07$ \\
\hline NM_014750 & DLG7 & 2.9 & UP & $5.17 \mathrm{E}-05$ \\
\hline U61166 & ITSN1 & 2.9 & UP & 4.23E-07 \\
\hline NM_001889 & CRYZ & 2.9 & UP & $6.10 \mathrm{E}-07$ \\
\hline NM_054033 & FKBP1B & 2.9 & UP & $1.02 \mathrm{E}-05$ \\
\hline NM_002725 & PRELP & 2.9 & UP & $1.94 \mathrm{E}-04$ \\
\hline NM_032800 & C1orf198 & 2.9 & UP & $1.82 \mathrm{E}-04$ \\
\hline NM_004512 & GALT & 2.9 & UP & $1.81 \mathrm{E}-05$ \\
\hline NM_020166 & MCCC1 & 2.9 & UP & 5.93E-06 \\
\hline AK027351 & RHOJ & 2.9 & UP & $2.36 \mathrm{E}-04$ \\
\hline NM_005576 & LOXL1 & 2.9 & UP & $8.88 \mathrm{E}-04$ \\
\hline NM_004701 & CCNB2 & 3.0 & UP & $3.60 \mathrm{E}-04$ \\
\hline AK025371 & ASAHL & 3.0 & UP & $1.06 \mathrm{E}-05$ \\
\hline NM_002430 & NM_002430.2 & 3.0 & UP & 2.03E-05 \\
\hline NM_003246 & THBS1 & 3.0 & UP & $5.19 \mathrm{E}-07$ \\
\hline NM_006307 & SRPX & 3.0 & UP & $1.17 \mathrm{E}-06$ \\
\hline AK023999 & $\mathrm{NA}$ & 3.0 & UP & $5.18 \mathrm{E}-05$ \\
\hline NM_032674 & LRRFIP1 & 3.0 & UP & $8.04 \mathrm{E}-06$ \\
\hline NM_004663 & RAB11A & 3.0 & UP & $2.22 \mathrm{E}-06$ \\
\hline NM_030926 & ITM2C & 3.0 & UP & $3.04 \mathrm{E}-07$ \\
\hline AK057721 & NA & 3.0 & UP & $1.86 \mathrm{E}-05$ \\
\hline NM_005786 & TSHZ1 & 3.0 & UP & 1.87E-04 \\
\hline NM_004538 & NAP1L3 & 3.0 & UP & $6.33 \mathrm{E}-06$ \\
\hline NM_032348 & MXRA8 & 3.0 & UP & 7.87E-07 \\
\hline NM_004404 & 37500 & 3.1 & UP & $5.66 \mathrm{E}-06$ \\
\hline NM_000253 & MTTP & 3.1 & UP & 1.65E-06 \\
\hline NM_052947 & ALPK2 & 3.1 & UP & 4.83E-06 \\
\hline AB011110 & RASA4 & 3.1 & UP & $2.54 \mathrm{E}-07$ \\
\hline NM_052932 & TMEM123 & 3.1 & UP & $8.35 \mathrm{E}-07$ \\
\hline NM_006822 & RAB40B & 3.1 & UP & $2.73 \mathrm{E}-06$ \\
\hline AK025953 & MYLK & 3.1 & UP & $1.80 \mathrm{E}-04$ \\
\hline NM_015976 & SNX7 & 3.1 & UP & 4.23E-07 \\
\hline AB037750 & SORCS2 & 3.1 & UP & $1.52 \mathrm{E}-05$ \\
\hline AB067801 & QKI & 3.1 & UP & 4.20E-06 \\
\hline NM_052969 & RPL39L & 3.1 & UP & $2.21 \mathrm{E}-06$ \\
\hline NM_004349 & RUNX1T1 & 3.2 & UP & $3.22 \mathrm{E}-05$ \\
\hline NM_020190 & OLFML3 & 3.2 & UP & $5.80 \mathrm{E}-04$ \\
\hline NM_005733 & $\mathrm{KIF} 20 \mathrm{~A}$ & 3.2 & UP & $6.34 \mathrm{E}-04$ \\
\hline AK057865 & THY1 & 3.2 & UP & $1.32 \mathrm{E}-04$ \\
\hline NM_024745 & SHCBP1 & 3.2 & UP & $2.83 \mathrm{E}-05$ \\
\hline NM_004642 & CDK2AP1 & 3.2 & UP & $1.21 \mathrm{E}-07$ \\
\hline NM_001998 & FBLN2 & 3.3 & UP & 4.57E-04 \\
\hline NM_002736 & PRKAR2B & 3.3 & UP & 1.65E-07 \\
\hline NM_012413 & QPCT & 3.3 & UP & $8.02 \mathrm{E}-06$ \\
\hline NM_001512 & GSTA4 & 3.3 & UP & 2.23E-07 \\
\hline NM_016240 & SCARA3 & 3.3 & UP & $4.58 \mathrm{E}-06$ \\
\hline NM_004480 & FUT8 & 3.4 & UP & 4.02E-06 \\
\hline AK024475 & PLEKHG4 & 3.4 & UP & 1.60E-07 \\
\hline AF043897 & hsa-mir-23b & 3.4 & UP & $4.49 \mathrm{E}-07$ \\
\hline NM_003070 & SMARCA2 & 3.4 & UP & $3.18 \mathrm{E}-06$ \\
\hline AK026784 & ITGBL1 & 3.4 & UP & $8.35 \mathrm{E}-05$ \\
\hline AL136842 & CDC42EP3 & 3.4 & UP & $3.59 \mathrm{E}-05$ \\
\hline NM_005195 & Q504X4_HUMAN & 3.4 & UP & $6.59 \mathrm{E}-04$ \\
\hline NM_001150 & ANPEP & 3.4 & UP & $5.96 \mathrm{E}-05$ \\
\hline BC012625 & PPP1R3C & 3.5 & UP & $4.58 \mathrm{E}-08$ \\
\hline NM_001864 & COX7A1 & 3.5 & UP & $8.28 \mathrm{E}-06$ \\
\hline AK057501 & CMTM2 & 3.5 & UP & $1.60 \mathrm{E}-06$ \\
\hline BC015134 & THBS1 & 3.5 & UP & $1.14 \mathrm{E}-07$ \\
\hline NM_000177 & GSN & 3.5 & UP & $7.89 \mathrm{E}-07$ \\
\hline M62896 & NA & 3.5 & UP & $1.17 \mathrm{E}-05$ \\
\hline
\end{tabular}




\begin{tabular}{|c|c|c|c|c|}
\hline NM_006986 & MAGED1 & 3.5 & UP & $1.01 \mathrm{E}-08$ \\
\hline NM_001067 & P11388-3 & 3.5 & UP & 8.01E-05 \\
\hline AK021982 & DYM & 3.5 & UP & $2.38 \mathrm{E}-07$ \\
\hline NM_002380 & MATN2 & 3.5 & UP & $1.21 \mathrm{E}-05$ \\
\hline AL137734 & NA & 3.5 & UP & $9.09 \mathrm{E}-05$ \\
\hline AK025909 & EFNA5 & 3.6 & UP & $5.99 \mathrm{E}-04$ \\
\hline NM_001822 & CHN1 & 3.6 & UP & 2.95E-07 \\
\hline AK027276 & ATXN7L4 & 3.6 & UP & $3.74 \mathrm{E}-04$ \\
\hline NM_019105 & P22105-2 & 3.6 & UP & $2.82 \mathrm{E}-05$ \\
\hline NM_030786 & SYNC1 & 3.6 & UP & $2.02 \mathrm{E}-08$ \\
\hline NM_031302 & GLT8D2 & 3.7 & UP & 3.05E-09 \\
\hline BC013971 & HOXA10 & 3.7 & UP & $2.51 \mathrm{E}-07$ \\
\hline NM_000961 & PTGIS & 3.7 & UP & $9.22 \mathrm{E}-04$ \\
\hline NM_020404 & CD248 & 3.7 & UP & $9.70 \mathrm{E}-09$ \\
\hline NM_001312 & CRIP2 & 3.8 & UP & $9.99 \mathrm{E}-06$ \\
\hline NM_021730 & Q96AM0_HUMAN & 3.8 & UP & $8.55 \mathrm{E}-09$ \\
\hline NM_024812 & BAALC & 3.9 & UP & 7.52E-07 \\
\hline NM_001449 & FHL1 & 3.9 & UP & $2.24 \mathrm{E}-04$ \\
\hline AB046796 & K1576_HUMAN & 3.9 & UP & 1.27E-04 \\
\hline NM_002527 & NTF3 & 4.0 & UP & 3.36E-07 \\
\hline NM_032048 & EMILIN2 & 4.0 & UP & $1.06 \mathrm{E}-06$ \\
\hline NM_001311 & CRIP1 & 4.0 & UP & $2.59 \mathrm{E}-05$ \\
\hline NM_032623 & NP_116012.2 & 4.0 & UP & $3.51 \mathrm{E}-04$ \\
\hline AF444143 & SPG3A & 4.0 & UP & 7.23E-05 \\
\hline NM_000165 & GJA1 & 4.1 & UP & $8.20 \mathrm{E}-04$ \\
\hline AK022997 & BCM:bcm4083 & 4.1 & UP & 2.34E-07 \\
\hline AL359558 & MCC & 4.2 & UP & $5.23 \mathrm{E}-08$ \\
\hline AK000776 & ROR1 & 4.2 & UP & $7.60 \mathrm{E}-05$ \\
\hline NM_007286 & SYNPO & 4.3 & UP & $5.81 \mathrm{E}-07$ \\
\hline AL050137 & OLFML2B & 4.3 & UP & $1.22 \mathrm{E}-09$ \\
\hline NM_001920 & $\mathrm{DCN}$ & 4.3 & UP & $9.24 \mathrm{E}-06$ \\
\hline NM_016824 & ADD3 & 4.4 & UP & $9.32 \mathrm{E}-08$ \\
\hline NM_005491 & CXorf6 & 4.4 & UP & $1.06 \mathrm{E}-09$ \\
\hline NM_005704 & PTPRU & 4.5 & UP & $2.53 \mathrm{E}-04$ \\
\hline NM_004098 & EMX2 & 4.6 & UP & $5.04 \mathrm{E}-08$ \\
\hline NM_014762 & DHCR24 & 4.6 & UP & $6.59 \mathrm{E}-05$ \\
\hline AB002365 & KIAA0367 & 4.7 & UP & 4.55E-07 \\
\hline NM_001129 & AEBP1 & 4.7 & UP & $5.32 \mathrm{E}-04$ \\
\hline NM_003783 & B3GALT2 & 4.8 & UP & 4.56E-04 \\
\hline AF156100 & HMCN1 & 4.8 & UP & $3.36 \mathrm{E}-07$ \\
\hline AK055710 & EBF3 & 4.8 & UP & $2.85 \mathrm{E}-05$ \\
\hline NM_003247 & THBS2 & 4.8 & UP & $5.36 \mathrm{E}-05$ \\
\hline NM_006206 & PDGFRA & 4.9 & UP & $2.38 \mathrm{E}-08$ \\
\hline NM_002160 & TNC & 4.9 & UP & $4.91 \mathrm{E}-06$ \\
\hline U97105 & DPYSL2 & 5.0 & UP & $5.09 \mathrm{E}-09$ \\
\hline NM_002048 & GAS1 & 5.0 & UP & $2.40 \mathrm{E}-06$ \\
\hline NM_000090 & COL3A1 & 5.1 & UP & 2.43E-07 \\
\hline NM_030965 & ST6GALNAC5 & 5.2 & UP & $2.70 \mathrm{E}-06$ \\
\hline NM_052880 & NP_443112.2 & 5.2 & UP & 4.06E-06 \\
\hline NM_021637 & TMEM35 & 5.2 & UP & $8.63 \mathrm{E}-04$ \\
\hline NM_006308 & HSPB3 & 5.5 & UP & 8.84E-06 \\
\hline NM_002966 & S100A10 & 5.6 & UP & $2.68 \mathrm{E}-06$ \\
\hline NM_002961 & S100A4 & 5.7 & UP & 1.19E-05 \\
\hline AK025346 & NA & 5.8 & UP & $2.59 \mathrm{E}-06$ \\
\hline AK026424 & GNG2 & 6.3 & UP & 3.01E-05 \\
\hline NM_002276 & KRT19 & 6.4 & UP & $4.69 \mathrm{E}-06$ \\
\hline NM_016206 & BCM:NM_016206 & 6.5 & UP & $5.82 \mathrm{E}-08$ \\
\hline NM_021111 & RECK & 6.6 & UP & $5.60 \mathrm{E}-08$ \\
\hline NM_001753 & CAV1 & 6.9 & UP & 4.01E-07 \\
\hline AK022198 & GALNT5 & 6.9 & UP & $1.84 \mathrm{E}-07$ \\
\hline NM_000095 & COMP & 7.4 & UP & $2.30 \mathrm{E}-05$ \\
\hline AL137578 & CTA-109P11.3 & 8.3 & UP & 4.23E-07 \\
\hline
\end{tabular}


CHAPTER 5

\begin{tabular}{llrll} 
AF216077 & TNFRSF19 & 8.4 & UP & $5.75 \mathrm{E}-07$ \\
AF245505 & MXRA5 & 8.9 & UP & $1.14 \mathrm{E}-07$ \\
NM_002982 & CCL2 & 9.4 & UP & $1.68 \mathrm{E}-09$ \\
NM_016557 & NA & 10.5 & UP & $2.89 \mathrm{E}-07$ \\
NM_000088 & COL1A1 & 10.8 & UP & $5.23 \mathrm{E}-08$ \\
AK021484 & SYNPO2 & 11.5 & UP & $2.70 \mathrm{E}-06$ \\
NM_000681 & ADRA2A & 12.7 & UP & $1.43 \mathrm{E}-08$ \\
NM_000089 & COL1A2 & 13.6 & UP & $1.03 \mathrm{E}-10$ \\
AB067499 & Q96PX6_HUMAN & 13.9 & UP & $3.13 \mathrm{E}-06$ \\
AB032953 & ODZ2 & 14.0 & UP & $2.14 \mathrm{E}-11$ \\
\hline
\end{tabular}


Supplementary table S3: Significantly differentially expressed genes for the 97 versus 70 comparison

\begin{tabular}{|c|c|c|c|c|}
\hline Genbank accession No. & Gene symbol & Fold-change & Direction & $\mathrm{p}$-value \\
\hline NM_002727 & PRG1 & 23.4 & DOWN & $5.23 \mathrm{E}-10$ \\
\hline NM_020169 & LXN & 8.9 & DOWN & $1.76 \mathrm{E}-08$ \\
\hline NM_001562 & IL18 & 8.0 & DOWN & $8.12 \mathrm{E}-07$ \\
\hline BC012486 & KRA24_HUMAN & 7.2 & DOWN & $3.16 \mathrm{E}-05$ \\
\hline NM_000600 & IL6 & 6.0 & DOWN & $5.53 \mathrm{E}-08$ \\
\hline NM_017723 & NP_060193.2 & 5.3 & DOWN & $5.90 \mathrm{E}-04$ \\
\hline NM_001175 & ARHGDIB & 5.2 & DOWN & $1.21 \mathrm{E}-05$ \\
\hline AL137678 & C20orf50 & 4.9 & DOWN & 7.24E-07 \\
\hline AF213459 & EPHA3 & 4.7 & DOWN & $9.99 \mathrm{E}-06$ \\
\hline NM_000799 & EPO & 4.5 & DOWN & 2.65E-05 \\
\hline AC004010 & AMIGO2 & 4.5 & DOWN & $1.49 \mathrm{E}-05$ \\
\hline NM_002837 & PTPRB & 4.5 & DOWN & 8.45E-06 \\
\hline AK055249 & UCHL1 & 4.5 & DOWN & 2.62E-08 \\
\hline NM_018421 & TBC1D2 & 4.5 & DOWN & 2.14E-05 \\
\hline NM_001321 & CSRP2 & 4.4 & DOWN & $6.68 \mathrm{E}-04$ \\
\hline AK021874 & TGFB2 & 4.4 & DOWN & 7.33E-08 \\
\hline AB029000 & SULF1 & 4.3 & DOWN & 4.45E-05 \\
\hline NM_004181 & UCHL1 & 4.3 & DOWN & 7.87E-08 \\
\hline NM_004669 & CLIC3 & 4.1 & DOWN & $1.32 \mathrm{E}-04$ \\
\hline AB032947 & CADPS & 4.0 & DOWN & 2.13E-07 \\
\hline NM_001845 & COL4A1 & 3.6 & DOWN & $3.45 \mathrm{E}-05$ \\
\hline NM_012082 & ZFPM2 & 3.6 & DOWN & $5.98 \mathrm{E}-04$ \\
\hline NM_004335 & BST2 & 3.3 & DOWN & 5.44E-04 \\
\hline NM_005070 & SLC4A3 & 3.3 & DOWN & 4.35E-05 \\
\hline AK021486 & BMP5 & 3.2 & DOWN & 4.35E-05 \\
\hline AF111170 & NUDT14 & 3.2 & DOWN & 5.65E-06 \\
\hline NM_003280 & TNNC1 & 3.1 & DOWN & $6.95 \mathrm{E}-05$ \\
\hline L10374 & Q5W0J2_HUMAN & 3.1 & DOWN & $1.04 \mathrm{E}-05$ \\
\hline NM_004010 & $\mathrm{DMD}$ & 3.1 & DOWN & 3.13E-04 \\
\hline NM_004862 & LITAF & 3.0 & DOWN & 4.07E-04 \\
\hline NM_031438 & NUDT12 & 3.0 & DOWN & 2.91E-04 \\
\hline NM_033642 & FGF13 & 3.0 & DOWN & 8.19E-04 \\
\hline NM_001553 & IGFBP7 & 3.0 & DOWN & $1.06 \mathrm{E}-04$ \\
\hline NM_000120 & EPHX1 & 2.9 & DOWN & $1.54 \mathrm{E}-08$ \\
\hline NM_031289 & GSG1 & 2.9 & DOWN & 7.42E-04 \\
\hline NM_002561 & $\mathrm{P} 2 \mathrm{R} \times 5$ & 2.8 & DOWN & $3.56 \mathrm{E}-05$ \\
\hline NM_000804 & FOLR3 & 2.8 & DOWN & 3.85E-05 \\
\hline NM_023927 & GRAMD3 & 2.8 & DOWN & 4.89E-05 \\
\hline NM_014578 & RHOD & 2.7 & DOWN & 3.01E-05 \\
\hline AK055506 & VGCNL1 & 2.7 & DOWN & $1.74 \mathrm{E}-05$ \\
\hline NM_020038 & $\mathrm{ABCC} 3$ & 2.7 & DOWN & $6.63 \mathrm{E}-04$ \\
\hline U60873 & NA & 2.7 & DOWN & 2.40E-04 \\
\hline NM_032603 & LOXL3 & 2.7 & DOWN & $1.47 \mathrm{E}-05$ \\
\hline NM_012099 & CD3EAP & 2.6 & DOWN & 2.22E- 07 \\
\hline NM_018214 & LRRC1 & 2.6 & DOWN & $1.28 \mathrm{E}-06$ \\
\hline NM_014068 & PSORS1C1 & 2.6 & DOWN & $1.39 \mathrm{E}-05$ \\
\hline AF161383 & C6orf129 & 2.6 & DOWN & 4.39E-05 \\
\hline NM_014900 & COBLL1 & 2.6 & DOWN & $5.51 \mathrm{E}-04$ \\
\hline NM_022842 & CDCP1 & 2.6 & DOWN & 8.63E-04 \\
\hline NM_023935 & CT116_HUMAN & 2.5 & DOWN & 4.24E-05 \\
\hline NM_021938 & BRUNOL5 & 2.5 & DOWN & $5.22 \mathrm{E}-04$ \\
\hline NM_004047 & ATP6V0B & 2.5 & DOWN & 4.51E-05 \\
\hline AK056831 & ANR52_HUMAN & 2.5 & DOWN & $8.12 \mathrm{E}-07$ \\
\hline NM_014951 & ZNF365 & 2.5 & DOWN & $1.88 \mathrm{E}-05$ \\
\hline BC017996 & Q8WVI0_HUMAN & 2.5 & DOWN & $5.02 \mathrm{E}-06$ \\
\hline AK026761 & NA & 2.4 & DOWN & $8.96 \mathrm{E}-05$ \\
\hline AB037823 & CHGUT_HUMAN & 2.4 & DOWN & 2.01E-04 \\
\hline NM_004992 & MECP2 & 2.4 & DOWN & $1.72 \mathrm{E}-04$ \\
\hline NM_002640 & SERPINB8 & 2.4 & DOWN & $8.13 \mathrm{E}-06$ \\
\hline
\end{tabular}




\begin{tabular}{|c|c|c|c|c|}
\hline NM_032514 & MAP1LC3A & 2.4 & DOWN & 1.50E-04 \\
\hline NM_005584 & MAB21L1 & 2.4 & DOWN & 3.40E-04 \\
\hline NM_033448 & KRT71 & 2.4 & DOWN & $1.72 \mathrm{E}-04$ \\
\hline NM_004409 & DMPK & 2.3 & DOWN & $5.29 \mathrm{E}-06$ \\
\hline NM_003214 & TEAD3 & 2.3 & DOWN & 7.60E-06 \\
\hline AB040883 & Q9H994_HUMAN & 2.3 & DOWN & 4.90E-04 \\
\hline AF109681 & ITGA11 & 2.3 & DOWN & 2.14E-05 \\
\hline NM_016069 & TIM16_HUMAN & 2.3 & DOWN & $3.84 \mathrm{E}-07$ \\
\hline NM_003364 & UPP1 & 2.3 & DOWN & $1.06 \mathrm{E}-04$ \\
\hline NM_030674 & SLC38A1 & 2.3 & DOWN & $8.14 \mathrm{E}-04$ \\
\hline NM_018281 & ECHDC2 & 2.3 & DOWN & 1.74E-04 \\
\hline NM_015645 & MFRP & 2.2 & DOWN & 1.53E-04 \\
\hline NM_007263 & COPE & 2.2 & DOWN & $9.18 \mathrm{E}-04$ \\
\hline BC009849 & SDSL & 2.2 & DOWN & $1.84 \mathrm{E}-05$ \\
\hline AF131821 & MGLL & 2.2 & DOWN & $5.18 \mathrm{E}-05$ \\
\hline NM_000389 & CDKN1A & 2.2 & DOWN & 4.47E-05 \\
\hline NM_032340 & C6orf125 & 2.2 & DOWN & 5.29E-06 \\
\hline NM_018973 & DPM3 & 2.2 & DOWN & 4.78E-05 \\
\hline AB040959 & DFNB31 & 2.2 & DOWN & 2.33E-04 \\
\hline NM_005094 & SLC27A4 & 2.2 & DOWN & 3.07E-04 \\
\hline BC000737 & RGS4 & 2.2 & DOWN & $6.86 \mathrm{E}-04$ \\
\hline NM_018556 & SIRPG & 2.2 & DOWN & $9.53 \mathrm{E}-06$ \\
\hline AK025627 & MIT:HsG2201 & 2.2 & DOWN & $9.26 \mathrm{E}-05$ \\
\hline AK056388 & UBE2Q1 & 2.2 & DOWN & $3.54 \mathrm{E}-04$ \\
\hline NM_017874 & CT027_HUMAN & 2.1 & DOWN & 1.50E-04 \\
\hline NM_016732 & RALY & 2.1 & DOWN & $9.18 \mathrm{E}-04$ \\
\hline NM_003491 & ARD1A & 2.1 & DOWN & $2.64 \mathrm{E}-05$ \\
\hline AL080219 & RTCD1 & 2.1 & DOWN & $9.74 \mathrm{E}-05$ \\
\hline NM_002314 & LIMK1 & 2.1 & DOWN & $1.28 \mathrm{E}-05$ \\
\hline BC009493 & DOLPP1 & 2.1 & DOWN & $1.71 \mathrm{E}-05$ \\
\hline NM_024038 & C19orf43 & 2.1 & DOWN & $2.48 \mathrm{E}-04$ \\
\hline NM_007100 & ATP5I & 2.1 & DOWN & 4.39E-05 \\
\hline NM_002413 & MGST2 & 2.1 & DOWN & 7.17E-05 \\
\hline NM_021170 & HES4 & 2.1 & DOWN & 3.03E-04 \\
\hline AL136826 & RGMA & 2.1 & DOWN & $5.44 \mathrm{E}-04$ \\
\hline NM_003893 & LDB1 & 2.1 & DOWN & 2.14E-05 \\
\hline NM_001029 & RPS26P10 & 2.1 & DOWN & 1.06E-04 \\
\hline NM_021570 & BARX1 & 2.1 & DOWN & 2.08E-04 \\
\hline NM_006368 & CREB3 & 2.1 & DOWN & 3.55E-05 \\
\hline NM_002069 & GNAI1 & 2.1 & DOWN & 3.84E-04 \\
\hline NM_017767 & SLC39A4 & 2.1 & DOWN & 7.58E-04 \\
\hline NM_003494 & DYSF & 2.1 & DOWN & $5.29 \mathrm{E}-05$ \\
\hline NM_003815 & ADAM15 & 2.0 & DOWN & $1.20 \mathrm{E}-04$ \\
\hline AK026750 & GPR20 & 2.0 & DOWN & $3.68 \mathrm{E}-04$ \\
\hline NM_003732 & EIF4EBP3 & 2.0 & DOWN & 4.04E-04 \\
\hline AJ272212 & PSKH1 & 2.0 & DOWN & $2.30 \mathrm{E}-05$ \\
\hline NM_032350 & NP_115726.1 & 2.0 & DOWN & 4.14E-04 \\
\hline NM_018438 & FBX06 & 2.0 & DOWN & $5.18 \mathrm{E}-05$ \\
\hline NM_031232 & APBA2BP & 2.0 & DOWN & 7.20E-05 \\
\hline NM_000964 & RARA & 2.0 & DOWN & 3.95E-04 \\
\hline NM_003313 & TSTA3 & 2.0 & DOWN & 2.83E-04 \\
\hline NM_006284 & TAF10 & 2.0 & DOWN & 4.24E-05 \\
\hline AB020649 & PLEKHM2 & 2.0 & DOWN & $8.18 \mathrm{E}-04$ \\
\hline NM_031484 & MARVELD1 & 2.0 & DOWN & $6.35 \mathrm{E}-04$ \\
\hline NM_001725 & BPI & 2.0 & DOWN & 1.06E-04 \\
\hline NM_005032 & PLS3 & 2.0 & UP & 4.49E-04 \\
\hline NM_033116 & NEK9 & 2.0 & UP & $9.65 \mathrm{E}-05$ \\
\hline NM_006915 & RP2 & 2.0 & UP & $2.02 \mathrm{E}-04$ \\
\hline NM_001358 & DHX15 & 2.0 & UP & 2.21E-04 \\
\hline AK056809 & NA & 2.0 & UP & $9.19 \mathrm{E}-05$ \\
\hline AK024475 & PLEKHG4 & 2.0 & UP & $8.51 \mathrm{E}-05$ \\
\hline D42055 & NEDD4 & 2.0 & UP & $8.66 \mathrm{E}-05$ \\
\hline
\end{tabular}




\begin{tabular}{|c|c|c|c|c|}
\hline NM_006931 & SLC2A3 & 2.0 & UP & $2.54 \mathrm{E}-05$ \\
\hline NM_003205 & TCF12 & 2.0 & UP & 4.00E-05 \\
\hline AB018353 & UNC84A & 2.1 & UP & $3.92 \mathrm{E}-04$ \\
\hline NM_003692 & C9orf30 & 2.1 & UP & 4.07E-04 \\
\hline AK023607 & SASH1 & 2.1 & UP & 7.79E-05 \\
\hline ВС017972 & SRGAP2P1 & 2.1 & UP & $4.50 \mathrm{E}-05$ \\
\hline NM_014799 & HEPH & 2.1 & UP & 4.07E-04 \\
\hline AF061733 & NP_689482.1 & 2.1 & UP & $1.29 \mathrm{E}-04$ \\
\hline AK002085 & CCNT1 & 2.1 & UP & $5.05 \mathrm{E}-05$ \\
\hline AK022008 & NOTCH2 & 2.1 & UP & 4.72E-04 \\
\hline NM_032832 & LRP11 & 2.1 & UP & 7.85E-06 \\
\hline AB058733 & TXNDC10 & 2.1 & UP & 4.94E-05 \\
\hline NM_005610 & RBBP4 & 2.1 & UP & $1.20 \mathrm{E}-04$ \\
\hline NM_002822 & TWF1 & 2.1 & UP & $4.78 \mathrm{E}-05$ \\
\hline NM_007286 & SYNPO & 2.1 & UP & $6.10 \mathrm{E}-04$ \\
\hline NM_002736 & PRKAR2B & 2.1 & UP & $3.90 \mathrm{E}-05$ \\
\hline AB051551 & LRRCC1 & 2.1 & UP & $8.92 \mathrm{E}-04$ \\
\hline AK001478 & RHOU & 2.1 & UP & 4.33E-05 \\
\hline NM_016121 & KCTD3 & 2.1 & UP & $3.16 \mathrm{E}-04$ \\
\hline NM_003278 & CLEC3B & 2.1 & UP & $9.24 \mathrm{E}-04$ \\
\hline AK024356 & socs6 & 2.1 & UP & $1.10 \mathrm{E}-05$ \\
\hline NM_002430 & NM_002430.2 & 2.2 & UP & $5.44 \mathrm{E}-04$ \\
\hline AB037748 & FAM44A & 2.2 & UP & 7.17E-05 \\
\hline AB046794 & FAM29A & 2.2 & UP & $1.29 \mathrm{E}-04$ \\
\hline AL132665 & BNIP3L & 2.2 & UP & $7.79 \mathrm{E}-05$ \\
\hline NM_005637 & SS18 & 2.2 & UP & $9.18 \mathrm{E}-04$ \\
\hline AF156100 & HMCN1 & 2.2 & UP & $5.71 \mathrm{E}-04$ \\
\hline NM_000179 & MSH6 & 2.2 & UP & $4.82 \mathrm{E}-04$ \\
\hline NM_004354 & CCNG2 & 2.2 & UP & $3.82 \mathrm{E}-05$ \\
\hline AB051516 & NP_444270.2 & 2.2 & UP & $1.29 \mathrm{E}-05$ \\
\hline AL080186 & C6orf111 & 2.2 & UP & 4.20E-05 \\
\hline AK057180 & C16orf45 & 2.2 & UP & $9.26 \mathrm{E}-05$ \\
\hline NM_001788 & 39326 & 2.2 & UP & $9.58 \mathrm{E}-05$ \\
\hline NM_000861 & $\mathrm{HRH} 1$ & 2.2 & UP & $2.89 \mathrm{E}-05$ \\
\hline AK056791 & MUC16 & 2.3 & UP & $1.32 \mathrm{E}-04$ \\
\hline AJ420481 & AOF1 & 2.3 & UP & $5.19 \mathrm{E}-04$ \\
\hline AK054863 & $A B \mid 2$ & 2.3 & UP & 4.39E-05 \\
\hline NM_006346 & C13orf24 & 2.3 & UP & $8.92 \mathrm{E}-06$ \\
\hline NM_022826 & MARCH7 & 2.3 & UP & 7.09E-05 \\
\hline NM_006417 & IFI44 & 2.3 & UP & $1.09 \mathrm{E}-05$ \\
\hline BC000195 & METTL9 & 2.3 & UP & 2.66E-04 \\
\hline AL136680 & GBP3 & 2.3 & UP & 3.85E-05 \\
\hline D50926 & MORC3 & 2.3 & UP & $1.50 \mathrm{E}-06$ \\
\hline NM_022831 & NA & 2.3 & UP & $4.90 \mathrm{E}-04$ \\
\hline NM_006264 & PTPN13 & 2.3 & UP & $6.90 \mathrm{E}-04$ \\
\hline AB040929 & CNTN3 & 2.4 & UP & $1.19 \mathrm{E}-04$ \\
\hline NM_000956 & PTGER2 & 2.4 & UP & $7.58 \mathrm{E}-04$ \\
\hline NM_000846 & GSTA1 & 2.4 & UP & $3.08 \mathrm{E}-05$ \\
\hline NM_005493 & RANBP9 & 2.4 & UP & $1.32 \mathrm{E}-04$ \\
\hline NM_004098 & EMX2 & 2.4 & UP & $3.90 \mathrm{E}-05$ \\
\hline AL161952 & GLUL & 2.4 & UP & $2.88 \mathrm{E}-04$ \\
\hline NM_001937 & DPT & 2.4 & UP & 8.63E-04 \\
\hline AF208850 & PTP4A2 & 2.4 & UP & $1.19 \mathrm{E}-04$ \\
\hline NM_018422 & Q7L3Y3_HUMAN & 2.4 & UP & $2.80 \mathrm{E}-05$ \\
\hline AL137938 & PXK & 2.4 & UP & $3.45 \mathrm{E}-05$ \\
\hline AK057721 & NA & 2.5 & UP & $1.50 \mathrm{E}-04$ \\
\hline NM_000930 & PLAT & 2.5 & UP & $2.79 \mathrm{E}-04$ \\
\hline U83115 & AlM1 & 2.5 & UP & $3.91 \mathrm{E}-05$ \\
\hline NM_033664 & $\mathrm{CDH} 11$ & 2.5 & UP & $1.34 \mathrm{E}-05$ \\
\hline NM_007150 & ZNF185 & 2.5 & UP & $9.18 \mathrm{E}-04$ \\
\hline NM_019105 & P22105-2 & 2.5 & UP & $6.10 \mathrm{E}-04$ \\
\hline AF177377 & EML4 & 2.5 & UP & $1.85 \mathrm{E}-05$ \\
\hline
\end{tabular}




\begin{tabular}{|c|c|c|c|c|}
\hline NM_016824 & ADD3 & 2.6 & UP & 2.72E-05 \\
\hline BC008377 & EPB41L3 & 2.6 & UP & $3.19 \mathrm{E}-05$ \\
\hline NM_012413 & QPCT & 2.6 & UP & $9.58 \mathrm{E}-05$ \\
\hline NM_024609 & NES & 2.6 & UP & 8.37E-04 \\
\hline AK023999 & NA & 2.6 & UP & $1.90 \mathrm{E}-04$ \\
\hline BC013592 & DDIT4L & 2.6 & UP & 4.07E-04 \\
\hline AF245505 & MXRA5 & 2.6 & UP & 7.42E-04 \\
\hline NM_003368 & USP1 & 2.6 & UP & 5.29E-06 \\
\hline NM_004369 & COL6A3 & 2.6 & UP & 4.14E-04 \\
\hline NM_052954 & CYYR1 & 2.7 & UP & $1.48 \mathrm{E}-04$ \\
\hline AB033056 & PPFIBP1 & 2.7 & UP & $3.74 \mathrm{E}-05$ \\
\hline AK021807 & LRP11 & 2.7 & UP & $1.84 \mathrm{E}-05$ \\
\hline AL049321 & NP_060404.3 & 2.7 & UP & $6.26 \mathrm{E}-06$ \\
\hline AB020689 & TBC1D9 & 2.7 & UP & $8.78 \mathrm{E}-06$ \\
\hline NM_032813 & TMTC4 & 2.7 & UP & $6.78 \mathrm{E}-05$ \\
\hline NM_024642 & GALNT12 & 2.8 & UP & 2.16E-05 \\
\hline NM_030926 & ITM2C & 2.8 & UP & $1.28 \mathrm{E}-06$ \\
\hline NM_007038 & ADAMTS5 & 2.8 & UP & 4.56E-04 \\
\hline NM_024745 & SHCBP1 & 2.8 & UP & $9.26 \mathrm{E}-05$ \\
\hline NM_005630 & SLCO2A1 & 2.8 & UP & 2.33E-05 \\
\hline NM_017515 & SLC35F2 & 2.8 & UP & $6.05 \mathrm{E}-04$ \\
\hline NM_024633 & CN139_HUMAN & 2.9 & UP & $3.90 \mathrm{E}-08$ \\
\hline NM_001067 & P11388-3 & 2.9 & UP & $3.71 \mathrm{E}-04$ \\
\hline NM_016206 & BCM:NM_016206 & 2.9 & UP & 4.45E-05 \\
\hline NM_014246 & CELSR1 & 3.0 & UP & $3.85 \mathrm{E}-04$ \\
\hline NM_006307 & SRPX & 3.0 & UP & $1.61 \mathrm{E}-06$ \\
\hline NM_032048 & EMILIN2 & 3.1 & UP & $1.69 \mathrm{E}-05$ \\
\hline NM_015310 & PSD3 & 3.1 & UP & 3.85E-05 \\
\hline NM_005864 & EFS & 3.2 & UP & 3.67E-05 \\
\hline NM_016557 & NA & 3.2 & UP & $5.30 \mathrm{E}-04$ \\
\hline NM_002160 & TNC & 3.3 & UP & $1.21 \mathrm{E}-04$ \\
\hline NM_007063 & TBC1D8 & 3.4 & UP & $1.19 \mathrm{E}-04$ \\
\hline AK000776 & ROR1 & 3.4 & UP & $3.46 \mathrm{E}-04$ \\
\hline NM_004480 & FUT8 & 3.4 & UP & 5.29E-06 \\
\hline NM_001920 & $\mathrm{DCN}$ & 3.4 & UP & $5.97 \mathrm{E}-05$ \\
\hline NM_021738 & SVIL & 3.5 & UP & 8.99E-06 \\
\hline NM_006622 & PLK2 & 3.5 & UP & 5.92E-05 \\
\hline NM_001311 & CRIP1 & 3.5 & UP & $7.79 \mathrm{E}-05$ \\
\hline NM_024812 & BAALC & 3.6 & UP & 2.47E-06 \\
\hline AK000933 & CHML & 3.6 & UP & $1.46 \mathrm{E}-08$ \\
\hline AL137578 & CTA-109P11.3 & 3.6 & UP & 1.18E-04 \\
\hline NM_030965 & ST6GALNAC5 & 3.7 & UP & 4.39E-05 \\
\hline AK022997 & BCM:bcm4083 & 3.7 & UP & $8.62 \mathrm{E}-07$ \\
\hline AK025909 & EFNA5 & 3.7 & UP & 3.87E-04 \\
\hline BC013939 & NA & 3.8 & UP & $5.29 \mathrm{E}-06$ \\
\hline NM_016240 & SCARA3 & 3.8 & UP & $1.97 \mathrm{E}-06$ \\
\hline AB020710 & EHBP1 & 3.9 & UP & $1.94 \mathrm{E}-07$ \\
\hline NM_001998 & FBLN2 & 3.9 & UP & $1.14 \mathrm{E}-04$ \\
\hline NM_021111 & RECK & 3.9 & UP & 5.06E-06 \\
\hline AK055243 & CCBE1 & 4.0 & UP & $3.19 \mathrm{E}-06$ \\
\hline NM_006206 & PDGFRA & 4.1 & UP & $1.69 \mathrm{E}-07$ \\
\hline NM_020190 & OLFML3 & 4.1 & UP & 7.65E-05 \\
\hline NM_001831 & CLU & 4.2 & UP & 7.60E-06 \\
\hline NM_000609 & CXCL12 & 4.3 & UP & $6.26 \mathrm{E}-06$ \\
\hline NM_057159 & EDG2 & 4.6 & UP & 3.90E-09 \\
\hline AK055710 & EBF3 & 5.0 & UP & 2.95E-05 \\
\hline AF216077 & TNFRSF19 & 5.0 & UP & $1.88 \mathrm{E}-05$ \\
\hline NM_001753 & CAV1 & 5.3 & UP & $3.69 \mathrm{E}-06$ \\
\hline AB002365 & KIAA0367 & 6.1 & UP & 7.87E-08 \\
\hline NM_000627 & LTBP1 & 6.4 & UP & 4.81E-05 \\
\hline NM_002380 & MATN2 & 6.6 & UP & $1.27 \mathrm{E}-07$ \\
\hline NM_003243 & TGFBR3 & 6.7 & UP & $2.61 \mathrm{E}-10$ \\
\hline
\end{tabular}




\begin{tabular}{lllll} 
NM_030781 & COLEC12 & 6.7 & UP & $5.46 E-04$ \\
NM_007029 & STMN2 & 7.0 & UP & $8.99 E-05$ \\
NM_001769 & CD9 & 7.2 & UP & $1.96 E-09$ \\
NM_002852 & PTX3 & 8.4 & UP & $2.64 E-08$ \\
\hline
\end{tabular}





\section{CHAPTER 6}

General Discussion 


\section{Diagnosis of OXPHOS disorders}

A genetic diagnosis of oxidative phosphorylation (OXPHOS) disorders is with some exceptions difficult to make because of the clinical and genetic heterogeneity. A single mutation can lead to a variety of clinical symptoms and syndromes, and the same phenotype can also be caused by a number of different mutations. The current genetic diagnostic procedures are often symptom based protocols, in which the clinical, pathological and biochemical manifestations are used to select the genes which are most likely to contain the pathogenic mutation. Leigh syndrome with a cytochrome c oxidase (COX) deficiency is an example where these phenotypic characteristics provide an indication of the genotypic defect. Tiranti et al. demonstrated that in Leigh syndrome with COX deficiency $75 \%$ of the patients had a mutation in the SURF1 gene, resulting in loss of function of the SURF1 protein [7, 63]. Although another study reported a lower frequency of $26 \%$ [64], more Leigh syndrome patients with COX deficiency have been genetically diagnosed with mutations in the SURF1 gene [6, 176-179], illustrating that Leigh syndrome associated with COX deficiency is a good indication of a genetic defect in the SURF1 gene. Leber Hereditary Optic Atrophy (LHON) is another example of an OXPHOS disorder with a direct relation between clinical symptoms and genetic defect, since three primary mtDNA mutations have been shown to be present in over $90 \%$ of the affected families [180]. When such a direct link between phenotype and genotype does not exist, a number of relatively common mitochondrial DNA (mtDNA) mutations and, dependent on the phenotype, mutations in nuclear genes are usually screened. Unfortunately, this approach is often inconclusive and can be costly and time consuming if multiple genes have to be tested.

Specific approaches have been developed and tested to make the identification of the genetic defects easier. The first distinction is between mutations in the mtDNA or in the nDNA. A clear maternal transmission of the disease will point to an mtDNA mutation and consanguinity or paternal transmission to a nuclear gene defect, but the available information or number of patients is not always sufficient to draw an unambiguous conclusion. Measurement of enzyme activities does not resolve the mutation origin directly, although the knowledge whether or not complex II, which is completely of nuclear origin, is involved, may provide a clue. Complementation studies by fusing patient fibroblast cells with mtDNA-less $\left(\rho^{0}\right)$ cells can provide a definite answer whether the genetic defect lies in the mtDNA or the nDNA. For example, fibroblasts of a patient with a severe mtDNA depletion were enucleated and complemented with a human-derived $\rho^{\circ}$ cell line. The hybdrid cells were able to grow on culture medium lacking uridine and pyruvate, indicating that the origin of the mtDNA depletion had to be under the control of the nuclear genome [181]. Tiranti et al. identified the chromosome on which the genetic defect resided in Leigh syndrome patients with cytochrome $c$ deficiency by chromosome transfer technology [7]. This, in combination with linkage analysis, lead to the identification of mutations in the SURF1 gene. However, this approach is technically demanding, costly and time consuming 
and will only work for patients who have fibroblast cells expressing the OXPHOS deficiency. In case sub chromosomal mapping is not possible by linkage analysis, then this has to be done by complementation analysis using chromosomal fragments. In another preselection method, OXPHOS complex assembly by SDS-PAGE or Blue Native-PAGE immunoblotting is studied. This can provide information about genetic defects in structural genes or genes involved in the assembly of the OXPHOS complexes. OXPHOS disorders characterised by complex I deficiency are one of the fields in which this technique appears to be promising $[182,183]$. These studies showed that a specific decrease of intact mitochondrial complex III is indicative for mutations in NDUFS2 and NDUFS4. Mutations in NDUFS4 appeared to be further related to decreased levels of fully assembled complex I combined with high levels of a defective sub complex not present in controls.

Classification of patients by their gene expression profile is evaluated as an alternative approach. However, this appears to be difficult due to the small number of patients available for classification experiments to find a classification signature. In one study, differences in expression were shown in cell lines with complex I defects as a result of genetic defects in different complex I genes [184], but a specific gene expression signature for complex I defects related to specific mutations was not reported. Despite the numerous methods described above to facilitate the genetic diagnosis of OXPHOS disorders, other approaches to improve and speed up genetic diagnosis must be considered. The development of better mutation screening techniques is a necessary alternative. Furthermore, more knowledge about the molecular pathological processes involved in OXPHOS disorders will be of great value in the genetic diagnosis of OXPHOS disorders.

Because $25 \%$ of all paediatric OXPHOS patients is expected to have a mutation in the mtDNA [23] and because the ability to discriminate between mtDNA and nDNA is important for genetic counselling, mutation screening of the entire mtDNA will be an essential step, complementing the methods mentioned above. In Chapter 2, a chip-based resequencing method was tested to resequence the entire mtDNA of patients with OXPHOS disorders. The method appeared to be very robust, delivering an average call rate of $94 \%$, which was comparable to reports from Affymetrix and others [33]. Heteroplasmy levels could be detected in a range from 5 to $50 \%$. Although the call rate was relatively high, it needs to be further improved for a diagnostic setting [185]. Furthermore, the sensitivity of heteroplasmy detection was lower than heteroduplex based methods like denaturing high-performance liquid chromatography (dHPLC). Optimisation of the data analysis procedure and software tools will most likely make it possible to reach detection levels in the same range. For example, we have observed a better performance in signal calling for the reverse strand compared to the forward strand on the MitoChip. It is not clear if this is a characteristic for specific regions in the mtDNA, but the generation of more data will gradually provide more insight into the region specific performance of the MitoChip, which must be considered when optimizing the data analysis. The signals from the forward and reverse strand on the MitoChip should be evaluated separately and should be incorporated in the analysis having 
a different weight specific for particular mtDNA regions. Several approaches are currently considered and several companies are working on this [185].

High-throughput sequencing of the complete mtDNA cannot be dealt with without interpretation issues. Many nucleotide variations are being detected, which in most cases are neutral polymorphisms. However, a substantial proportion could be pathogenic nucleotide variations. To evaluate the pathogenic properties of unknown nucleotide variations, calculation of a pathogenicity score as described by Mitchell et al. for complex I mutations may help to decide whether a nucleotide variation is likely to be pathogenic [50]. A slightly adapted version of this scoring scheme has been applied to primary pathogenic mutations in the mRNA coding region [186]. A similar scoring scheme exists for mitochondrial tRNA mutations [60]. However, calculation of such scores appears not to be straightforward, because not always all data is available and it remains for a large part circumstantial evidence. Moreover, it should be repeated regularly to cover the new genomic information which is being produced at an extremely rapid pace. This indicates that there is a need for a standardised diagnostic flowchart in which, if possible, all aspects of the scoring mechanism are integrated, including automated database searches as well as easy functional assays. An initiative for the former has already been taken by the MitoCircle project (EU grant, Sixth Framework Program, contr. no. 005260), which started with the development of standardised diagnostic guidelines for OXPHOS disorders and the development of a standardised database, in which phenotypic information together with mtDNA genetic variations, both pathogenic and unclassified variants, is being listed [22]. Further expansion of the database by including complete mtDNA sequences will eventually facilitate genetic diagnosis and improve prognostic predictions. Initial MitoChip screening of 28 patients with OXPHOS disease resulted in the identification of three known pathogenic mutations and four new unclassified variants which were likely to be pathogenic (Chapter 2). Meanwhile, one of the four latter variants has been reported to be the pathogenic mtDNA mutation in a family with a prevalent ocular phenotype [187]. This illustrates that results which might initially seem to be inconclusive have to be followed up and reviewed periodically. Furthermore, this shows that $25 \%$ of all paediatric OXPHOS patients can be genetically diagnosed with this technique. At the same time, this technique can be used to exclude mtDNA involvement to a certain level, although the number of unclassified variants (a mean value of three to four per patients) should be taken into account. Additionally, chip-based resequencing could also be valuable for the candidate gene mutation screening approach, for example to screen all known structural genes or assembly factors for (one of the complexes of) the respiratory genes. This is for example being done in a diagnostic setting for Inherited syndromes of intrahepatic cholestasis [188]. Such an approach might be much more favourable for resolving the genetic basis of OXPHOS disorders than any of the genotype-phenotype based approaches.

The development of next-generation sequencers, with faster and cheaper sequencing techniques, like the 454-sequencing system (Roche), the Solexa sequencing system (Illumina) or the SOLID 
system (Applied Biosystems), brings this approach even closer. These systems are able to sequence millions of bases per hour and are based on massive parallel single template sequencing. A sequence by synthesis strategy is used in the 454 and Solexa where round by round nucleotides are added which results in a fluorescent signal (Solexa) or a luciferase light emission signal (454) which can be detected and recorded. The 454 sequencing system is able to yield relatively large DNA sequences (250-350 bp) compared to the shorter fragments ( $<40 \mathrm{bp}$ ) with the Solexa system. In both systems, a reference sequence is used to align and compare the sequenced sample fragments to in order to determine nucleotide variations, although the 454 can also be used for de novo sequencing. The complexity of the human genome hampers these techniques from being used for high throughput mutation screening. Long-range $P C R$ and an enrichment step of the preferred sequences from the genomic DNA prior to sequencing provide a solution for this problem, e.g. by selecting the exons of interest for resequencing. Recently, the use of microarray based techniques has been reported to enrich genomic target sequences of interest [189-191]. In short, these techniques use probes on a microarray directed against specific genomic targets (e.g. exons). Genomic DNA is fragmented and hybridised to the array, after which the array is washed to remove a-specifically bound targets. Subsequently, the hybridised targets are eluted from the microarray and amplified after which the sample is ready for sequencing. Another publication described the use of a microarray to synthesise target specific oligonucleotide probes. After synthesis, the probes are released from the array to capture the genomic sequences of interest. After capturing, amplification was carried out of the captured targets resulting in a circular product, after which all linear and thus non-target sequences were removed by restriction digestion [192]. Using these techniques, between 6,000 and 200,000 exons could be enriched for subsequent sequencing, bringing the application of these technologies for molecular genetic diagnostics nearby.

A drawback of these techniques is the huge amount of data generated, i.e. images have to be saved and quantified after each sequencing round, which has to be analyzed. Specialised software will be necessary for analysis and a huge storage capacity is needed for data storage. Furthermore, screening thousands of enriched genomic targets will deliver an enormous amount of unknown nucleotide variations, which will make the interpretation about pathogenicity of a variation difficult and which will lead to considerable uncertainty. This will be the trade-off for the increasing number of cases for which an accurate diagnosis can be made. This emphasises again the need to store all nucleotide variations in a central database and a strong need for easy, fast, and cheap functional assays to confirm pathogenicity. Up until now, there are no reports of these techniques being capable of the detection of heteroplasmic nucleotide variations in the mtDNA, although they are being used for ultra-deep sequencing of pathology specimens $[193,194]$ and detection of heteroplasmic mutations with a low mutation load should be possible. Although the cost per nucleotide has become about 100-fold cheaper compared to traditional sequencing methods [195], the required coverage for reaching a reliable $1 \%$ detection level may make this approach too expensive compared to alternatives. Comparing chip-based resequencing with 
these massive parallel sequencers, it appears that the chip is more appropriate to rapidly screen many genes for individual cases, whereas massive parallel sequencing is more effective for large number of patients and genes.

As an alternative to large-scale sequencing, simple, cheap and sensitive pre-screening methods may still be considered. A high-throughput method which currently is able to detect heteroplasmic mtDNA mutations at a sensitivity level comparable to dHPLC is the high-resolution melting analysis (Lightscanner Idaho), which is able to analyze 96 samples in about 10 minutes [196-198]. Although this technique is based on heteroduplex analysis, detection of homoplasmic variations is directly possible, probably with lesser sensitivity, or as heteroduplex after annealing to a wild-type reference sample. It has even been reported that homozygous variations with a nearest neighbour thermodynamic symmetry, i.e. the bases adjacent to the SNP are identical on both DNA strands and the SNP consists of an interchange between complementary bases, can be detected by high resolution melting analysis [199]. A second fast method for the detection of mtDNA variations is the mutation surveyor technique. This method is based on restriction enzyme digestion by a specific endonuclease which cleaves double stranded DNA at any mismatch site. Subsequently, products are separated and analyzed by agarose gel electrophoresis. Because mutation detection is based on the identification of differences in size of the digestion products, homoplasmic mutations can not be detected, but heteroplasmic mutations can be detected at mutation loads as low as $3 \%$ [200, 201]. In conclusion, the number of samples which will provide the opportunity for automated analysis, the possibilities of improving the data analysis, the costs of the chip itself, and the speed with which other new high throughput sequencing and mutation detection techniques are developed, will determine whether MitoChip resequencing of the mtDNA will prevail to be the preferred method for mtDNA resequencing for the next couple of years. Whether candidate gene screening by chip resequencing will become the preferred method for candidate gene screening will depend on the same factors. However, it is very likely that the solution for the genetic diagnosis of OXPHOS disorders will come from one of these technologies.

\section{Pathophysiology of OXPHOS disorders: patient derived and non-patient derived experimental models for gene expression profiling}

Microarray gene expression studies have proven their value in unravelling pathological processes underlying diseases and also in classifying patients groups using gene expression signatures. The microarray gene expression technique would be very wanted as an aid in the study of OXPHOS disorders. However, classification of patients with OXPHOS disease remains a challenge because of the limited availability of homogeneous patient groups due to the genetic and clinical heterogeneity. Furthermore, since OXPHOS defects usually affect tissues with a high energy demand like brain, heart, skeletal muscle, renal, and the endocrine system [32], it is necessary to 
obtain one of these tissues to perform diagnostic and experimental tests on. Most often skeletal muscle (needle) biopsies are used for diagnostic purposes. They are also the preferred tissue type to use for research purposes. Since the quantity of muscle biopsies is often limited, only a small fraction will be available for research. These limitations urged us to explore different approaches in order to use microarray gene expression profiling for the study of OXPHOS disorders, and experimental models have been used by us and others as an alternative, some of which will be discussed below.

\section{Non-patient derived experimental models for OXPHOS disorders and gene expression profiling}

Very often, animal models are used to simulate human disease conditions and to extrapolate results and conclusions to the human situation. Yeast, mouse, fly, and worm models have been used to study OXPHOS disorders. The advantage of the mouse as a model organism is its phylogenetically short distance to the human. Advantages of yeast, fly, and worm as model organisms are mainly the short life cycles of these organisms. They are relatively easy to work with, the genomes are fully sequenced, and the genetic manipulation is straightforward. It has been reported that mutations in a gene in yeast that is homologous gene to the human SURF1, the SHY1 (Surf Homolog of Yeast) gene, also results in cytochrome $c$ oxidase deficiency, just as in Leigh syndrome patients with mutations in the SURF1 gene [202]. The yeast SHY1 protein has a similar function to the human SURF1 protein [203] and the yeast SHY1-model has been used to investigate the role of SHY1 in the cytochrome c oxidase complex assembly [203-206]. A mousemodel for Leigh syndrome caused by mutations in the SURF1 gene has also been created [207] and the knock-out mouse had a phenotype very similar to that observed in the patients with a substantially decreased muscle strength and motor performance, isolated cytochrome $c$ oxidase deficiency in muscle, liver, heart and brain, and morphological abnormalities in skeletal muscle mainly reflected by COX negative muscle fibres and decrease mitochondrial proliferation. Despite the fact that numerous aspects resemble the human situation, a prominent characteristic of Leigh syndrome is unfortunately not present in this mouse knockout model, which is the characteristic neurological pathology of Leigh syndrome identified as focal, bilateral symmetrical necrotic lesions in the basal ganglia, thalamus, brainstem, and spinal cord.

Various other mouse models have been created, some of which have been reviewed by Wallace $[208,209]$. One of the models is the Tfam deficient mouse model which might be a good model to study mtDNA depletion syndrome in humans. These mice presented with systemic, cardiac, and pancreatic $\beta$-cell defects, probably as a result of mtDNA depletion. TFAM encodes a mitochondrial transcription factor and reduced levels of this protein has been shown to results in mtDNA depletion in human [210]. ANT1 deficient mice most importantly present with mitochondrial myopathy and hypertrophic cardiomyopathy [211]. The characteristics of the ANT1 knockout mice illustrate that these mice share pathology with patients with autosomal dominant progressive external ophthalmoplegia (adPEO, OMIM\#157640) and therefore might be a good model to study 
this mtDNA multiple deletion syndrome. Mouse models have also been created for a frequently affected nuclear gene in humans, which is the polymerase gamma gene. Mice were created containing a proof-reading deficient polymerase gamma (POLG) gene in the heart, which caused an accumulation of mtDNA mutations. The mitochondrial apoptotic process was activated and the mice developed dilated cardiomyopathy [212, 213]. In another study, transgenic mice were generated with a mutant polymerase gamma gene expressed in a neuron-specific manner in order to create a genetic model for bipolar disorder, and more specifically for the mitochondrial disease chronic progressive external ophthalmoplegia (CPEO). CPEO is often caused by a mutation in POLG, which results in the accumulation of mtDNA deletions. Just as in CPEO patients, transgenic mice produced mtDNA deletions and mutations in the brain [214].

The creation of mouse models with a defect in the mtDNA has shown to be extremely difficult and only few models exist. Chimeric mice have been created carrying the m.2433T $>C$ mutation in the $16 \mathrm{~S}$ rRNA gene leading to resistance to the mitochondrial ribosome inhibitor chloramphenicol (CAP). These CAP resistant chimeric mice presented with clinical features similar to OXPHOS disorders, i.e. congenital cataracts, mitochondrial myopathy and cardiomyopathy [215]. In a second model, mice were generated harbouring an mtDNA deletion. The deletion size was 4,696 bp and included 6 tRNA genes and 7 structural genes which also resulted in OXPHOS disorder phenotypes. Histochemical and morphological investigation of skeletal and heart muscle showed mosaic distribution of COX-negative and -positive cells with ragged-red fibre-like characteristics and blood lactate levels were elevated [216].

A variety of models are available for Drosophila, of which some are reviewed by Sánchez-Martínez et al. [217]. One of the models discussed in this review is a functional knock-down of the Surf1 gene in Drosophila [218]. Ubiquitous silencing of the Surf1 gene in Drosophila resulted in high mortality before entering pupariation, which resembles the high embryonic lethality of the SURF1 knock-out mice [207]. When silencing Surf1 in the central nervous system alone, larval muscle contained altered mitochondria. Furthermore, a decreased COX activity in cephalic sections could be shown. These abnormalities are characteristics of patients with Leigh syndrome caused by a SURF1 mutation. The first model in C. elegans was the target-selected mutagenesis of the nuo-1 gene, which encodes the 51-kDa active site subunit of complex I [219]. Mutants are characterised by behavioural defects indicative of muscular and/or neuronal defects, which is very similar to the human situation were mutations in the human homolog NDUFV1 result in myoclonic epilepsy, hypotonia, ataxia, Leigh syndrome, leukodystrophy, and other neurological conditions [220, 221]. The C. elegans mutant having a mutation in the mev1 gene, encoding a subunit of complex II, has been helpful in understanding the involvement of a defective electron transport chain in increased ROS production. Mev1 mutants have an increased ROS production and an abnormal energy metabolism [222]. 
Experimental animal models for OXPHOS disorders are very useful in gene expression studies, especially since they overcome the problem of the sample scarcity. Furthermore, working with animal models is much more flexible, e.g. animals can be exposed to conditions in favour of the experimental setup, and genetic manipulation is often relatively easy.

\section{Patient derived experimental models for gene expression profiling}

Animal models, having a homogeneous genetic background, not always represent the wild-type or pathological situation in human to the best extent, making extrapolation of the results difficult. Therefore, patient-derived model systems can be a better alternative from that perspective. It would be preferable to use the patient's primary tissue and the tissue in which the OXPHOS defect is most pronounced, like skeletal muscle, directly. Because of the limited availability of such material, cell cultures of patient cells can be used as an alternative. This has the advantage that taking blood for lymphocytes or a skin biopsy for fibroblast cells is less disturbing for patients compared to undergoing a muscle biopsy. Moreover, cells can be grown in large quantities and can be stored and reused for long periods of time. However, in vitro cultured cells are not the same as in vivo tissues and these cells are often not affected by the condition. In order to use cell culture systems to explore the molecular biological processes involved in the pathology of OXPHOS disorders, cultured cells have to suffer from the OXPHOS defect as well. One of the measurable manifestations can be a biochemical deficiency of one or more of the five complexes of the electron transport chain. Lymphocytes, primary skin fibroblasts, and myoblasts are the most prominent cell types which have been used as cell culture models to study OXPHOS disorders. Besides the use of primary patient cell cultures, primary cells can also be genetically manipulated to simulate OXPHOS defects. Osteosarcoma cell lines depleted of their mtDNA ( $\rho^{\circ}$ cells) transfected with mtDNA from patient cells are often used to create cells with identical nuclear genetic background, but different types of mtDNA mutations or heteroplasmy levels (cybrid cells).

There have been a number of microarray gene expression studies reporting the use of patientderived cell cultures to study OXPHOS disorders. Van der Westhuizen et al. studied complex I deficiencies using primary fibroblasts from patients with mutations in five different genes encoding complex I subunits. Because fibroblasts mainly rely on anaerobic energy metabolism (glycolysis) for their energy supply, they chose to compare gene expression of these cells under anaerobic culture conditions to aerobic (OXPHOS) culture conditions. One of the observations from this study was a significant induction of methallothioneins, which was believed to be related to their antioxidant function as a protection from an increase in ROS production [184]. In another study using microarray and realtime-PCR, matrix metalloproteinase 1 (MMP1) expression was shown to be significantly increased in cultured skin fibroblasts from patients with the myoclonic epilepsy and ragged-red fibres (MERRF) syndrome. Supported by the measurement of a significant increase in intracellular $\mathrm{H}_{2} \mathrm{O}_{2}$ concentration in these patient cells and previous reports 
in literature, the induction of MMP1 was related to an increase in ROS production [223]. In a study of Leber's hereditary optic neuropathy (LHON), microarray gene expression analysis was carried out on cybrid cells constructed from five patient cell lines containing LHON mtDNA mutations compared to controls cell lines. An important observation was that the majority of the gene expression changes was due to the $\rho^{\circ}$ and the cybridisation process, instead of resulting from LHON mtDNA mutations. Nevertheless, several significant gene expression changes could be identified, which were related to the presence of the LHON mtDNA mutations [224]. Jahangir Tafrechi et al. compared gene expression profiles of cybrid cells with $100 \%$ wild-type mtDNA, cybrid cells with $100 \% \mathrm{~m} .3243 \mathrm{~A}>\mathrm{G}$ mutant mtDNA, and $\rho^{\circ}$ cells. Their conclusion was that different mtDNA mutations result in distinct nuclear gene expression changes, suggesting the involvement of different molecular pathways, partly explaining the clinical heterogeneity observed in OXPHOS disorders [225]. Cortopassi et al. performed an extensive gene expression study in which they compared gene expression profiles from five mitochondrial diseases (LHON, Friedreich's ataxia, MELAS, Kearns-Sayre Syndrome, and NARP) using lymphoblasts, osteosarcomas, fibroblasts, myoblasts, differentiated muscle, undifferentiated and differentiated NT2 neural cells, and undifferentiated and differentiated $\mathrm{SH}_{-} \mathrm{SY}_{5} \mathrm{Y}$ neuroblastoma cells. Transcripts of the unfolded protein response and of the cell cycle pathway were commonly induced. Transcripts involved in vesicular secretion and protein synthesis were commonly reduced [226]. These studies show that a prominent process taking place in various OXPHOS disorders is an increased ROS production. The resulting ROS damage may lead to secondary responses like ROS defence, and changes in protein turnover and cell cycle related processes. A very important aspect when using cell culture models is that one must be very careful not to draw conclusions on the basis of artefacts.

As mentioned above, the effects of different mutation loads of mtDNA mutations can be studied in cybrid cells. A disadvantage is that these cell lines are predominantly tumour cells with a genetically modified and unstable nuclear background, introducing extra experimental variability, as illustrated by the study of Danielson et al. [224]. Another option is to use cell lines from different patients with varying mutation loads of the mtDNA mutation. The advantage is that these cells are not genetically manipulated, but the disadvantage of this method is the different nuclear backgrounds of each cell culture. The introduction of the extra variation by these two methods complicates the identification of the effect of the mtDNA mutation and the effect of the differences in mutation load. In chapter five, a cell culture model system is presented which circumvents these problems by exploiting the mutation load differences between single cultured fibroblast cells. Fibroblast cells from one single patient carrying the m.9176T $>C$ mutation in the ATP6 gene were cloned by seeding the cells in a very low concentration, allowing cell colonies to arise from only one single cell. This gave rise to various cell lines with the same genetic background, but with different m.9176T $>\mathrm{C}$ mutation loads. This system is the preferred method to study mutation load effects of the m.9176T>C mutation. The applicability of this cell culture model system for other mtDNA mutations depends on a number of factors. Firstly, as already mentioned previously, the OXPHOS defect has to be expressed in the cultured cells, e.g. it has to result in a 
biochemical phenotype. Secondly, the distribution of the mutation loads of individual fibroblast cells has to be broad, providing the possibility of cloning cells with varying mutation loads and preferably also with only wild-type mtDNA molecules. Finally, the mutation load has to be stable during the culturing procedure. For example, the common heterogeneous MELAS m.3243A>C mutation has a biochemical phenotype in cultured skin fibroblasts, and the mutation load is stable during culturing as long as an exogenous uridine supply is provided [227]. Single muscle fibres and single lymphocyte cells have been analyzed for this mutation and the widest mutation load ranges were from $12 \%$ to $100 \%$ for single muscle fibres and from $19 \%$ to $92 \%$ for single lymphocyte cells $[104,105,228]$. Single fibroblast cells unfortunately have not been analyzed, but it can be expected that fibroblast mutation loads are in the range of the muscle fibre and lymphocyte mutation loads. The MELAS m.3243A>G mutation most likely fulfils all criteria, and therefore is a good candidate for this cell culture model system, although a $0 \%$ mutation load monoclonal cell culture may be difficult to achieve. The cell culture model system might also be useful for the m.8993 $>\mathrm{G} / \mathrm{C}$ mutation in the ATP6 gene resulting in Neuropathy, Ataxia, and Retinitis Pigmentosa (NARP) or Leigh syndrome [9-11, 149]. This mutation has been shown to be stable with respect to its mutation load over cell culture passages and the mutation also results in a biochemical phenotype in cultured skin fibroblasts. However, the distribution of the mutation load in single fibroblast cells has not yet been determined, but since the characteristics of this mutation are very much like those of the m.9176T >C mutation in the same gene, it is plausible that this criterion will also be fulfilled.

The use of animal or cell culture model systems for gene expression studies of OXPHOS disorders are alternatives for the use of primary patient tissue, but have considerable pitfalls. Gene expression profiling of primary patient tissue remains to be the preferred approach, and validation of results from experimental models to the human in vivo situation always needs to be strived for.

\section{Molecular biological processes involved in OXPHOS disorders}

Besides improvement of genetic diagnosis for OXPHOS disorders (chapter 2) and the development of good experimental models in order to study OXPHOS disorders (chapter 5), this thesis shows that microarray gene expression technology has proven to be a valuable tool to study the molecular pathology of OXPHOS disorders and to identify (new) entries for therapeutic interventions. We were able to identify a number of known, but also novel molecular biological pathways related to the specific disorders under study, which may imply to other OXPHOS disorders as well. Moreover, microarray gene expression analysis led to the identification of gene expression markers for OXPHOS disease stages. Lastly, through the newly identified molecular biological pathways in the investigated OXPHOS disorders, new therapeutic possibilities were identified which are worthwhile for follow-up investigations. 
In chapters three and four, two microarray gene expression studies are described comparing skeletal muscle from Leigh syndrome patients with a mutation in the SURF1 gene and skeletal muscle from carriers of the MELAS m.3243A $>G$ mutation respectively with skeletal muscle from control subjects. To perform a more specific analysis for the m.3243A>G mutation, the m.3243A>C mutation carriers were divided into three groups, and each group was compared to the controls. One group comprised a-symptomatic mutation carriers (AS3243) and another consisted of symptomatic mutation carriers (S3243). A third group combined all mutation carriers (Mut3243), irrespective of having symptoms or not. In chapter five, a study is described in which monoclonal primary skin fibroblast originating from one patient are used for the investigation of gene expression differences between different mutation loads of the m.9176T>C mutation. The goal of these studies was to gain insight into the molecular biological processes involved in the pathology of these diseases, and, more specifically for the m.3243A>C and m.9176T $>C$ mutations, to investigate the effect of the mutation load on gene expression. Table 1 gives an overview of the most important processes which were identified as changed in the comparisons made in these studies.

\section{Energy pathways, OXPHOS, and mitochondrial biogenesis}

Because there is a defect in OXPHOS, which is one of the most important parts of the aerobic energy metabolism, one might expect that compensatory changes would occur in the expression of genes involved in other energy metabolism systems such as the glycolysis or the citric acid cycle. Furthermore, changes in the expression of OXPHOS related genes and genes involved in mitochondrial biogenesis would be expected to occur as well. However, only in muscle of asymptomatic m.3243A $>\mathrm{G}$ mutation carriers, we observed stimulation of OXPHOS and mitochondrial biogenesis, but no other changes in energy metabolism occurred. None of these expected compensatory mechanisms were identified in muscle of the symptomatic m.3243A $>\mathrm{G}$ mutation carriers, SURF1 Leigh patients, or in fibroblasts carrying the m.9176T $>C$ mutation. However, these results are in line with previous microarray gene expression studies on OXPHOS disorders. Van der Westhuizen et al. carried out a microarray gene expression study on cell lines from patients with complex I deficiency due to mutations in genes coding for different complex I subunits. The majority of these cell lines showed no change in gene expression of mtDNA encoded structural OXPHOS components when cultured under aerobic compared to anaerobic conditions [184]. In another study, gene expression of cybrid cells with $100 \%$ wild-type mtDNA, $100 \%$ mutant m.3243A>G mtDNA, and $\rho^{\circ}$ cells were compared using microarray gene expression. Similar to our results in muscle of symptomatic m.3243A>G mutation carriers, mostly having high mutation loads, the gene set 'oxidative phosphorylation' showed no change when the m.3243 mutant cells were compared to wild-type cells [225]. Furthermore, two other microarray gene expression studies have been reported on muscle biopsies from OXPHOS patients. One study investigated three MELAS patients carrying the m.3243A $<G$ mutation, and only in the patient with 
the highest mutation load (91\%) an induction of OPXHOS genes was identified [118]. The other study, in which also symptomatic MELAS subjects with the m.3243A<G mutation were studied, did not report any gene expression changes of OXPHOS genes [119].

On the basis of the reports in literature and from our results, we can conclude that stimulation of OXPHOS and mitochondrial biogenesis does not occur when pathology is present, but may contribute to prevention of a-symptomatic m.3243A $>\mathrm{G}$ mutation carriers becoming symptomatic. Mutations in the SURF1 gene and the m.9176T>C mutation in the ATP6 gene both result in deficiencies of electron transport chain complexes, i.e. the cytochrome $c$ oxidase complex and the ATP synthase subunit respectively. It is likely that these defects are more severe than the defect of the tRNA ${ }^{\text {Leu }}$ molecule caused by the m.3243A $>\mathrm{G}$ mutation, because they directly affect the energy production system, whereas the defective tRNA ${ }^{\text {Leu }}$ molecule indirectly affects the oxidative energy metabolism. In contrast to muscle, the fibroblast cells carrying the m.9176T>C mutation only show a biochemical phenotype. Therefore, these cells might have a different response than muscle cells. In conclusion, the stimulation of OXPHOS and mitochondrial biogenesis appears to be able to initially protect the cells carrying the m.3243A>C mutation, in contrast to cells with a SURF1 or the m.9176T>C mutation.

Protein turnover, ROS production, apoptosis, and complement activation

An increased protein turnover, characterised by increased protein synthesis and degradation, appears to be present in muscle cells from Leigh syndrome patients with a SURF1 mutation and in muscle cells from m.3243A $>C$ mutation carriers. An increase in protein turnover was previously reported when increased ubiquitin mRNA levels were found in MELAS m.3243A>G muscle cells [118]. However, this was not observed in affected m.3243A>G MELAS patients, which is in agreement with the findings in cybrid cells carrying $100 \%$ of the $\mathrm{m} .3243 \mathrm{~A}>\mathrm{G}$ mutation. In these cybrids, ribosomal protein gene expression was down-regulated and transcripts coding for proteins involved in ubiquitin-mediated proteolysis were also significantly down-regulated. The measurement of a decreased cytosolic protein synthesis rate in these cybrid cells confirmed the microarray results [225]. The explanation which was suggested for the decrease in protein synthesis was, that energy demanding processes like protein synthesis are down-regulated to compensate for the OXPHOS defect. A suppression of protein synthesis was also identified in a microarray study of fibroblasts carrying the m.8344A $>\mathrm{G}$ mutation in the $\mathrm{tRNA} \mathrm{A}^{\text {Lys }}$ gene resulting in myoclonic epilepsy and ragged red fibres (MERRF) syndrome [223]. In a study where 9 different cell types from five different OXPHOS disorders were analyzed by microarray gene expression, protein biosynthesis was identified as a changed process, however, in this study it was not explained whether the process was stimulated or inhibited [226].

In addition to the increases protein turnover, increased ROS production and complement activation are found in skeletal muscle from Leigh syndrome patients with a SURF1 mutation and 
in muscle from m.3243A>G mutation carriers and patients. Our hypothesis is that increased ROS production resulting from a defective OXPHOS system and a defect in the translation machinery in case of the m.3243A $>C$ mutation, results in oxidatively damaged and dysfunctional proteins. In an effort to replace the damaged and dysfunctional proteins, protein turnover is increased, but this apparently is insufficient, or in case of the m.3243A>G mutation still gives rise to dysfunctional proteins. When damage persists, increases, and compensation fails, muscle pathology arises, and in turn triggers the activation of apoptosis and complement components (see below). Not all of these changes were identified in fibroblasts with the m.9176T $>C$ mutation. ROS production and oxidative protein damage was not experimentally determined in these fibroblasts, but there were no gene expression changes related to ROS defence which could be indicative for an increased ROS production. Surprisingly, this is in contrast with previous reports on fibroblast cell cultures with OXPHOS defects [174, 223, 229]. In complex I deficient fibroblasts, an up-regulation of metallothioneins was identified, which is also an indication for increased ROS production since metallothioneins are have an antioxidant function [184]. Further study on our m.9176T $>C$ fibroblast cell cultures is needed in order to find an explanation for this discrepancy.

Table 1: Biological processes altered in muscle from Surf Leigh patients and m.3243A $>\mathrm{G}$ mutation carriers and in fibroblasts with the m.9176T $>\mathrm{C}$ mutation compared to controls

\begin{tabular}{|c|c|c|c|c|c|}
\hline \multirow[b]{2}{*}{ Process } & \multicolumn{4}{|c|}{ Skeletal Muscle } & \multirow{2}{*}{$\begin{array}{l}\text { Fibroblasts } \\
\text { m.9176T>C }\end{array}$} \\
\hline & SURF1 Leigh & MELAS AS3243 & MELAS S3243 & MELAS Mut3243 & \\
\hline Apoptosis & ND & $\uparrow$ & $\mathrm{NC}$ & $\uparrow \downarrow$ & $\uparrow \downarrow$ \\
\hline Protein synthesis & $\uparrow$ & $\uparrow \uparrow$ & $\mathrm{NC}$ & $\uparrow$ & NC \\
\hline Protein degradation & $\uparrow$ & $\uparrow$ & $\mathrm{NC}$ & ND & $\mathrm{NC}$ \\
\hline ROS production* & $\uparrow$ & $\uparrow$ & $\uparrow$ & - & $\mathrm{ND} / \mathrm{NC}^{* *}$ \\
\hline Oxidative protein damage* & $\mathrm{NC}$ & $\uparrow$ & $\uparrow$ & - & ND \\
\hline OXPHOS & NC & $\uparrow$ & NC & ND & NC \\
\hline Mitochondrial biogenesis & ND & $\uparrow$ & NC & ND & NC \\
\hline Energy pathways & NC & NC & NC & NC & NC \\
\hline Complement system & $\uparrow$ & $\uparrow$ & $\uparrow \uparrow$ & ND & NC \\
\hline
\end{tabular}

ND: Not determined by the process based analysis, but some individual genes might be changed. NC: No change. $\uparrow:$ Stimulation of the process. $\downarrow$ : Inhibition of the process. For the determination of ROS production and oxidative protein damage, no general mutation effects were estimated, as is indicated by a dash (-).

* ROS production and oxidative protein damage were determined through a DHE-staining and an oxprot assay (see chapters three and four for assay details).

${ }^{* *}$ Not determined by DHE-staining and no change according to the gene expression information.

Apoptosis has been identified as an altered process in skeletal muscle of m.3243A $>\mathrm{C}$ mutation carriers. Also the monoclonal fibroblasts carrying the m.9176T $>C$ mutation showed apoptotic changes in comparison with fibroblasts containing only wild-type mtDNA. For the m.3243A>C mutation carriers, a stimulation of apoptosis was mainly identified in a-symptomatic mutation carriers, whereas both stimulation and inhibition of the process occurred in mutation carriers irrespective of the presence of symptoms or not. No apoptotic changes were identified for the 
m.3243A $>$ G symptomatic mutation carriers. This illustrates that there appears to be a transition during disease progression from a-symptomatic to symptomatic from stimulation to inhibition of the apoptotic process. An explanation for these changes might be related to the progression of the disease, where in the a-symptomatic mutation carriers, stimulation of apoptosis appears to be a rescue mechanism in order to remove the damaged cells containing the dysfunctional and damaged proteins due to the increased ROS production. In combination with protein turnover and the complement activation (see below), stimulating muscle regeneration, this forms the coping mechanism for cells carrying the m.3243A $>\mathrm{G}$ mutation. However, when damage increases, this mechanism apparently does not hold up or requires too much energy, and apoptosis is inhibited in combination with a stronger stimulation of the complement system as the sole final rescue attempt. The situation is probably similar in the monoclonal fibroblast carrying the m.9176T>C mutation. In these cells, apoptosis appeared to be both stimulated as inhibited, which might reflect different cell populations in a different phase of the process. The gene expression changes were all on the level of regulation. No actual apoptosis effector genes were changed in expression, which indicates that actual apoptosis was not occurring. Further studies will be necessary to explain the role of these apoptotic changes with respect to the m.9176T $>C$ mutation and its pathological consequences.

In chapters three and four it was demonstrated that activation of complement in the skeletal muscle from the investigated patients does not lead to cell lyses by the membrane attack complex (MAC), which is the final step in the classical complement pathway. Furthermore it was shown that according to the gene expression information it is not the result of infiltration of inflammatory cells like B-lymphocytes or macrophages. Complement activation in the muscle of patients having these two OXPHOS disorders most likely is an effort to stimulate muscle regenerative processes, as complement has been shown to be able to stimulate tissue and organ regeneration $[78-80,106]$. One might argue that this complement activation is purely a reaction to the muscle pathology, and that it is not specific to these disorders. However, comparison of data from Duchene muscular dystrophy (DMD) patients, who suffer from severe muscle pathology, with data from SURF1 Leigh patients illustrated that activation of complement components most likely has different objectives in these two disorders. In DMD, the activation of complement is probably triggered by the infiltration of inflammatory cells and results in necrosis of the muscle fibres. In contrast, we have illustrated that complement activation in muscle from SURF1 Leigh patients is not the results of infiltrating inflammatory cells and that muscle fibre necrosis is not occurring (chapter 3). Changes in gene expression and immunohistochemical data have provided support for the hypothesis that complement activation most likely is an effort to stimulate muscle fibre regeneration and development in SURF1 Leigh patients (chapter 3), and probably also in subjects carrying the MELAS m.3243A>C mutation. Further study on skeletal muscle of other OXPHOS disorders will have to show whether the complement activation is specific to the muscle pathology in general, or to these two OXPHOS disorders or maybe OXPHOS disorders in general. The expression of complement components in muscle, and possibly complement component $\mathrm{C}_{3}$ 
in particular, in combination with the protein turnover rate, may be a marker for the severity and progression of the OXPHOS disorders.

\section{Conclusions}

This thesis describes the successful application of a chip-based resequencing method to facilitate genetic diagnosis of OXPHOS disorders (chapter 2). The advantages of this approach demonstrate the feasibility to tackle mutation detection in genetically and clinically heterogeneous disorders by this or alternative high throughput sequencing technologies. It can be concluded that much can be gained from these new techniques, but that experimental methods and data analysis and interpretation have to be optimised. To study pathophysiological processes involved in OXPHOS disorders, a cell culture model system was proposed which allowed different mtDNA mutation loads to be compared without introducing experimental or genetic bias (chapter 5). This technique is the preferred method to study mtDNA mutation load differences in a genetically controlled environment. Chapters 3 and 4 describe two gene expression studies on skeletal muscle from Leigh syndrome patients with a SURF1 mutation and carriers of the m.3243A>C mutation compared to controls. This led to the identification of pathological processes which are directly or indirectly related to these OXPHOS disorders, some of which might provide new clues for the development of therapies for OXPHOS disorders. A number of rescue or coping mechanisms as a reaction to mutations in the nuclear encoded SURF1 gene and the mtDNA encoded tRNA ${ }^{\text {Leu1 }}$ gene $(\mathrm{m} .3243 \mathrm{~A}>\mathrm{G})$ and ATP6 gene ( $\left.\mathrm{m} .9176 \mathrm{~T}>\mathrm{C}\right)$ leading to Leigh syndrome or MELAS were identified (chapters 3, 4, and 5 respectively). As OXPHOS components appear to be stimulated prior to disease manifestation, increasing energy capacity could provide similar effects. Preventing ROS damage to proteins, DNA and tissue by preventing excessive ROS production, but also improving ROS protection, seems to be important. When protein and/or tissue damage occurs after all, stimulating protein turnover and supplying the required energy capacity might be helpful in order to compensate for the defects and postpone disease manifestation. Our studies have provided solid evidence to explore further routes of intervention along these lines. 


\section{References}

1. Anderson, S., et al., Sequence and organization of the human mitochondrial genome. Nature, 1981. 290(5806): p. 457-65.

2. Jukes, T.H. and S. Osawa, The genetic code in mitochondria and chloroplasts. Experientia, 1990. 46(11-12): p. 1117-26.

3. Campos, Y., et al., Leigh syndrome associated with the T9176C mutation in the ATPase 6 gene of mitochondrial DNA. Neurology, 1997. 49(2): p. 595-7.

4. Dionisi-Vici, C., et al., Fulminant Leigh syndrome and sudden unexpected death in a family with the T9176C mutation of the mitochondrial ATPase 6 gene. J Inherit Metab Dis, 1998. 21(1): p. 2-8.

5. Makino, M., et al., Confirmation that a T-to-C mutation at 9176 in mitochondrial DNA is an additional candidate mutation for Leigh's syndrome. Neuromuscul Disord, 1998. 8(3-4): p. 149-51.

6. Pequignot, M.O., et al., Mutations in the SURF1 gene associated with Leigh syndrome and cytochrome C oxidase deficiency. Hum Mutat, 2001. 17(5): p. 374-81.

7. Tiranti, V., et al., Mutations of SURF-1 in Leigh disease associated with cytochrome coxidase deficiency. Am J Hum Genet, 1998.63(6): p. 1609-21.

8. Zhu, Z., et al., SURF1, encoding a factor involved in the biogenesis of cytochrome c oxidase, is mutated in Leigh syndrome. Nat Genet, 1998. 20(4): p. 337-43.

9. Makela-Bengs, P., et al., Correlation between the clinical symptoms and the proportion of mitochondrial DNA carrying the 8993 point mutation in the NARP syndrome. Pediatr Res, 1995. 37(5): p. 634-9.

10. Tatuch, Y., et al., The 8993 mtDNA mutation: heteroplasmy and clinical presentation in three families. Eur J Hum Genet, 1994. 2(1): p. 35-43.

11. White, S.L., et al., Genetic counseling and prenatal diagnosis for the mitochondrial DNA mutations at nucleotide 8993. Am J Hum Genet, 1999. 65(2): p. 474-82.

12. Matthews, P.M., et al., Comparison of the relative levels of the 3243 (A-->G) mtDNA mutation in heteroplasmic adult and fetal tissues. J Med Genet, 1994 - 31(1): p. 41-4.

13. Vilarinho, L., et al., The mitochondrial A3243G mutation presenting as severe cardiomyopathy. J Med Genet, 1997.34(7): p. 607-9.

14. van den Ouweland, J.M., et al., Mutation in mitochondrial tRNA(Leu)(UUR) gene in a large pedigree with maternally transmitted type II diabetes mellitus and deafness. Nat Genet, 1992. 1(5): p. 368-71.

15. Reardon, W., et al., Diabetes mellitus associated with a pathogenic point mutation in mitochondrial DNA. Lancet, 1992. 340(8832): p. 1376-9.

16. Schulz, J.B., et al., Mitochondrial gene mutations and diabetes mellitus. Lancet, 1993. 341(8842): p. 438-9.

17. Manouvrier, S., et al., Point mutation of the mitochondrial tRNA(Leu) gene (A $3243 \mathrm{G}$ ) in maternally inherited hypertrophic cardiomyopathy, diabetes mellitus, renal failure, and sensorineural deafness. J Med Genet, 1995. 32(8): p. 654-6.

18. Goto, Y., I. Nonaka, and S. Horai, A mutation in the tRNA(Leu)(UUR) gene associated with the MELAS subgroup of mitochondrial encephalomyopathies. Nature, 1990. 348(6302): p. 651-3.

19. Kobayashi, Y., et al., A point mutation in the mitochondrial tRNA(Leu)(UUR) gene in MELAS (mitochondrial myopathy, encephalopathy, lactic acidosis and stroke-like episodes). Biochem Biophys Res Commun, 1990. 173(3): p. 816-22.

20. Enter, C., et al., A specific point mutation in the mitochondrial genome of Caucasians with MELAS. Hum Genet, 1991. 88(2): p. 233-6. 
21. Ciafaloni, E., et al., MELAS: clinical features, biochemistry, and molecular genetics. Ann Neurol, 1992. 31(4): p. 391-8.

22. Brandon, M.C., et al., MITOMAP: a human mitochondrial genome database--2004 update. Nucleic Acids Res, 2005. 33(Database issue): p. D611-3.

23. Thorburn, D.R., Mitochondrial disorders: prevalence, myths and advances. J Inherit Metab Dis, 2004. 27(3): p. 349-62.

24. Valnot, I., et al., A mutation in the human heme A:farnesyltransferase gene (COX10) causes cytochrome c oxidase deficiency. Hum Mol Genet, 2000. 9(8): p. 1245-9.

25. Venter, J.C., et al., The sequence of the human genome. Science, 2001. 291(5507): p. 1304-51.

26. McPherson, J.D., et al., A physical map of the human genome. Nature, 2001. 409(6822): p. 934-41.

27. Golub, T.R., et al., Molecular classification of cancer: class discovery and class prediction by gene expression monitoring. Science, 1999. 286(5439): p. 531-7.

28. van 't Veer, L.J., et al., Gene expression profiling predicts clinical outcome of breast cancer. Nature, 2002. 415(6871): p. 530-6.

29. Mootha, V.K., et al., PGC-1alpha-responsive genes involved in oxidative phosphorylation are coordinately downregulated in human diabetes. Nat Genet, 2003 . 34(3): p. 267-73.

30. Eijssen, L.M.T., Analysis of microarray gene expression data sets. Department of Genetics and Cell Biology, Cardiovascular Research Institute Maastricht (CARIM). Maastricht University. Maastricht, 2006.

31. Chinnery, P.F., New approaches to the treatment of mitochondrial disorders. Reprod Biomed Online, 2004. 8(1): p. 16-23.

32. Wallace, D.C., Mitochondrial diseases in man and mouse. Science, 1999. 283(5407): p. 14828.

33. Maitra, A., et al., The Human MitoChip: a high-throughput sequencing microarray for mitochondrial mutation detection. Genome Res, 2004. 14(5): p. 812-9.

34. Jakupciak, J.P., et al., Mitochondrial DNA as a cancer biomarker. J Mol Diagn, 2005. 7(2): p. 258-67.

35. Mullenbach, R., P.J. Lagoda, and C. Welter, An efficient salt-chloroform extraction of DNA from blood and tissues. Trends Genet, 1989. 5(12): p. 391.

36. Comi, G.P., et al., Cytochrome c oxidase subunit I microdeletion in a patient with motor neuron disease. Ann Neurol, 1998. 43(1): p. 110-6.

37. Pease, A.C., et al., Light-generated oligonucleotide arrays for rapid DNA sequence analysis. Proc Natl Acad Sci U S A, 1994. 91(11): p. 5022-6.

38. Lipshutz, R.J., et al., High density synthetic oligonucleotide arrays. Nat Genet, 1999. 21(1 Suppl): p. 20-4.

39. Andrews, R.M., et al., Reanalysis and revision of the Cambridge reference sequence for human mitochondrial DNA. Nat Genet, 1999. 23(2): p. 147.

40. R Development Core Team. R: A language and environment for statistical computing. 2004. Vienna, Austria: R Foundation for Statistical Computing.

41. Shaag, A., et al., Mitochondrial encephalomyopathy associated with a novel mutation in the mitochondrial tRNA(leu)(UUR) gene (A3243T). Biochem Biophys Res Commun, 1997. 233(3): p. 637-9.

42. Kirby, D.M., et al., Mutations of the mitochondrial ND1 gene as a cause of MELAS. J Med Genet, 2004. 41(10): p. 784-9.

43. Santorelli, F.M., et al., Identification of a novel mutation in the mtDNA ND5 gene associated with MELAS. Biochem Biophys Res Commun, 1997. 238(2): p. 326-8.

44. Pulkes, T., et al., The mitochondrial DNA G13513A transition in ND5 is associated with a LHON/MELAS overlap syndrome and may be a frequent cause of MELAS. Ann Neurol, 1999. 46(6): p. 916-9.

45. Penisson-Besnier, I., et al., Recurrent brain hematomas in MELAS associated with an ND5 gene mitochondrial mutation. Neurology, 2000. 55(2): p. 317-8. 
46. Chol, M., et al., The mitochondrial DNA G13513A MELAS mutation in the NADH dehydrogenase 5 gene is a frequent cause of Leigh-like syndrome with isolated complex I deficiency. J Med Genet, 2003. 40(3): p. 188-91.

47. Kirby, D.M., et al., Low mutant load of mitochondrial DNA G13513A mutation can cause Leigh's disease. Ann Neurol, 2003. 54(4): p. 473-8.

48. Sudo, A., et al., Leigh syndrome caused by mitochondrial DNA G13513A mutation: frequency and clinical features in Japan. J Hum Genet, 2004. 49(2): p. 92-6.

49. Helm, M., et al., Search for characteristic structural features of mammalian mitochondrial tRNAs. Rna, 2000. 6(10): p. 1356-79.

50. Mitchell, A.L., et al., Sequence Variation In Mitochondrial Complex I Genes: Mutation Or Polymorphism? J Med Genet, 2006. 43(3): p. 175-9.

51. Suomalainen, A., et al., Use of single strand conformation polymorphism analysis to detect point mutations in human mitochondrial DNA. J Neurol Sci, 1992. 111(2): p. 222-6.

52. van Orsouw, N.J., et al., Mutational scanning of mitochondrial DNA by two-dimensional electrophoresis. Genomics, 1998. 52(1): p. 27-36.

53. van den Bosch, B.J., et al., Mutation analysis of the entire mitochondrial genome using denaturing high performance liquid chromatography. Nucleic Acids Res, 2000. 28(20): p. E89.

54. Bjorheim, J. and P.O. Ekstrom, Review of denaturant capillary electrophoresis in DNA variation analysis. Electrophoresis, 2005. 26(13): p. 2520-30.

55. Wartell, R.M., S.H. Hosseini, and C.P. Moran, Jr., Detecting base pair substitutions in DNA fragments by temperature-gradient gel electrophoresis. Nucleic Acids Res, 1990. 18(9): p. 2699-705.

56. Wong, L.J. and R.G. Boles, Mitochondrial DNA analysis in clinical laboratory diagnostics. Clin Chim Acta, 2005. 354(1-2): p. 1-20.

57. Sohm, B., et al., Towards understanding human mitochondrial leucine aminoacylation identity. J Mol Biol, 2003. 328(5): p. 995-1010.

58. Corona, P., et al., A novel mtDNA mutation in the ND5 subunit of complex I in two MELAS patients. Ann Neurol, 2001. 49(1): p. 106-10.

59. DiMauro, S. and E.A. Schon, Mitochondrial DNA mutations in human disease. Am J Med Genet, 2001. 106(1): p. 18-26.

60. McFarland, R., et al., Assigning pathogenicity to mitochondrial tRNA mutations: when "definitely maybe" is not good enough. Trends Genet, 2004. 20(12): p. 591-6.

61. Ingman, M. and U. Gyllensten, mtDB: Human Mitochondrial Genome Database, a resource for population genetics and medical sciences. Nucleic Acids Res, 2006. 34(Database issue): p. D749-51.

62. Dahl, H.H., Getting to the nucleus of mitochondrial disorders: identification of respiratory chain-enzyme genes causing Leigh syndrome. Am J Hum Genet, 1998. 63(6): p. 1594-7.

63. Tiranti, V., et al., Loss-of-function mutations of SURF-1 are specifically associated with Leigh syndrome with cytochrome c oxidase deficiency. Ann Neurol, 1999. 46(2): p. 161-6.

64. Sue, C.M., et al., Differential features of patients with mutations in two COX assembly genes, SURF-1 and SCO2. Ann Neurol, 2000. 47(5): p. 589-95.

65. Tiranti, V., et al., Characterization of SURF-1 expression and Surf-1p function in normal and disease conditions. Hum Mol Genet, 1999. 8(13): p. 2533-40.

66. Bruno, C., et al., A novel mutation in the SURF1 gene in a child with Leigh disease, peripheral neuropathy, and cytochrome-c oxidase deficiency. J Child Neurol, 2002. 17(3): p. 233-6.

67. den Dunnen, J.T. and S.E. Antonarakis, Mutation nomenclature extensions and suggestions to describe complex mutations: a discussion. Hum Mutat, 2000. 15(1): p. 7-12.

68. Gautier, L., et al., affy--analysis of Affymetrix GeneChip data at the probe level. Bioinformatics, 2004. 20(3): p. 307-15.

69. Lindsey, J.K., Models for Repeated Measurements. 1999, Oxford: Oxford University Press. 
70. Lindsey, J.K. $R$ modeling libraries. [cited; Available from: http://popgen.unimaas.nl/ jlindsey/rcode.html.

71. Dai, M., et al., Evolving gene/transcript definitions significantly alter the interpretation of GeneChip data. Nucleic Acids Res, 2005. 33(20): p. e175.

72. Akaike, H. Information theory and an extension of the maximum likelihood principle. in Second International Symposium on Inference Theory. 1973. Budapest: Akadémiai Kiadó.

73. Atkinson, A.C., A note on the generalized information criterion for choice of a model. Biometrika, 1980. 67: p. 413-418.

74. Dahlquist, K.D., et al., GenMAPP, a new tool for viewing and analyzing microarray data on biological pathways. Nat Genet, 2002. 31(1): p. 19-20.

75. Doniger, S.W., et al., MAPPFinder: using Gene Ontology and GenMAPP to create a global gene-expression profile from microarray data. Genome Biol, 2003. 4(1): p. R7.

76. Dennis, G., Jr., et al., DAVID: Database for Annotation, Visualization, and Integrated Discovery. Genome Biol, 2003. 4(5): p. P3.

77. Hsiao, L.L., et al., A compendium of gene expression in normal human tissues. Physiol Genomics, 2001. 7(2): p. 97-104.

78. Alamdari, D.H., et al., High sensitivity enzyme-linked immunosorbent assay (ELISA) method for measuring protein carbonyl in samples with low amounts of protein. Free Radic Biol Med, 2005. 39(10): p. 1362-7.

79. Zhao, H., et al., Superoxide reacts with hydroethidine but forms a fluorescent product that is distinctly different from ethidium: potential implications in intracellular fluorescence detection of superoxide. Free Radic Biol Med, 2003. 34(11): p. 1359-68.

80. Wilson, M.C., et al., Lactic acid efflux from white skeletal muscle is catalyzed by the monocarboxylate transporter isoform MCT3. J Biol Chem, 1998. 273(26): p. 15920-6.

81. Baker, S.K., M.A. Tarnopolsky, and A. Bonen, Expression of MCT1 and MCT4 in a patient with mitochondrial myopathy. Muscle Nerve, 2001. 24(3): p. 394-8.

82. Pilegaard, $\mathrm{H}$., et al., Effect of high-intensity exercise training on lactate/H+ transport capacity in human skeletal muscle. Am J Physiol, 1999. 276(2 Pt 1): p. E255-61.

83. Grune, T., T. Reinheckel, and K.J. Davies, Degradation of oxidized proteins in K562 human hematopoietic cells by proteasome. J Biol Chem, 1996. 271(26): p. 15504-9.

84. Esposito, L.A., et al., Mitochondrial disease in mouse results in increased oxidative stress. Proc Natl Acad Sci U S A, 1999. 96(9): p. 4820-5.

85. Walport, M.J., Complement. First of two parts. N Engl J Med, 2001. 344(14): p. 1058-66.

86. Song, W.C., M.R. Sarrias, and J.D. Lambris, Complement and innate immunity. Immunopharmacology, 2000. 49(1-2): p. 187-98.

87. Porter, J.D., et al., A chronic inflammatory response dominates the skeletal muscle molecular signature in dystrophin-deficient mdx mice. Hum Mol Genet, 2002. 11(3): p. 26372.

88. Turk, R., et al., Common pathological mechanisms in mouse models for muscular dystrophies. Faseb J, 2006. 20(1): p. 127-9.

89. Cornelio, F. and I. Dones, Muscle fiber degeneration and necrosis in muscular dystrophy and other muscle diseases: cytochemical and immunocytochemical data. Ann Neurol, 1984. 16(6): p. 694-701.

90. Engel, A.G. and G. Biesecker, Complement activation in muscle fiber necrosis: demonstration of the membrane attack complex of complement in necrotic fibers. Ann Neurol, 1982. 12(3): p. 289-96.

91. Gasque, P., et al., Human skeletal myoblasts spontaneously activate allogeneic complement but are resistant to killing. J Immunol, 1996. 156(9): p. 3402-11.

92. Legoedec, J., et al., Complement classical pathway expression by human skeletal myoblasts in vitro. Mol Immunol, 1997. 34(10): p. 735-41.

93. Mastellos, D. and J.D. Lambris, Complement: more than a 'guard' against invading pathogens? Trends Immunol, 2002. 23(10): p. 485-91. 
94. Del Rio-Tsonis, K., et al., Expression of the third component of complement, C3, in regenerating limb blastema cells of urodeles. J Immunol, 1998. 161(12): p. 6819-24.

95. Markiewski, M.M., et al., C3a and C3b activation products of the third component of complement (C3) are critical for normal liver recovery after toxic injury. J Immunol, 2004. 173(2): p. 747-54.

96. Strey, C.W., et al., The proinflammatory mediators C3a and C5a are essential for liver regeneration. J Exp Med, 2003. 198(6): p. 913-23.

97. van Dartel, M. and T.J. Hulsebos, Characterization of PMP22 expression in osteosarcoma. Cancer Genet Cytogenet, 2004. 152(2): p. 113-8.

98. Gutierrez, J., et al., Free radicals, mitochondria, and oxidized lipids: the emerging role in signal transduction in vascular cells. Circ Res, 2006. 99(9): p. 924-32.

99. Hammans, S.R., et al., The mitochondrial DNA transfer RNALeu(UUR) A-->G(3243) mutation. A clinical and genetic study. Brain, 1995. 118 ( Pt 3): p. 721-34.

100. Chinnery, P.F., et al., Molecular pathology of MELAS and MERRF. The relationship between mutation load and clinical phenotypes. Brain, 1997.120 ( Pt 10): p. 1713-21.

101. Torroni, A., et al., Mitochondrial DNA haplogroups do not play a role in the variable phenotypic presentation of the A3243G mutation. Am J Hum Genet, 2003. 72(4): p. 1005-12.

102. van Essen, E.H., et al., HLA-DQ polymorphism and degree of heteroplasmy of the A3243G mitochondrial DNA mutation in maternally inherited diabetes and deafness. Diabet Med, 2000. 17(12): p. 841-7.

103. Flierl, A., H. Reichmann, and P. Seibel, Pathophysiology of the MELAS 3243 transition mutation. J Biol Chem, 1997. 272(43): p. 27189-96.

104. Silvestri, G., et al., Single-fiber PCR in MELAS(3243) patients: correlations between intratissue distribution and phenotypic expression of the mtDNA(A3243G) genotype. Am J Med Genet, 2000. 94(3): p. 201-6.

105. Ozawa, M., I. Nonaka, and Y. Goto, Single muscle fiber analysis in patients with 3243 mutation in mitochondrial DNA: comparison with the phenotype and the proportion of mutant genome. J Neurol Sci, 1998. 159(2): p. 170-5.

106. van Empel, V.P., et al., Downregulation of apoptosis-inducing factor in harlequin mutant mice sensitizes the myocardium to oxidative stress-related cell death and pressure overloadinduced decompensation. Circ Res, 2005. 96(12): p. e92-e101.

107. Stefanovic, B., et al., TRAM2 protein interacts with endoplasmic reticulum $\mathrm{Ca2}+$ pump Sercazb and is necessary for collagen type I synthesis. Mol Cell Biol, 2004. 24(4): p. 1758-68.

108. Wanker, E.E., et al., Functional characterization of the $180-k D$ ribosome receptor in vivo. $J$ Cell Biol, 1995. 130(1): p. 29-39.

109. Liou, M.L. and H.C. Liou, The ubiquitin-homology protein, DAP-1, associates with tumor necrosis factor receptor (p60) death domain and induces apoptosis. J Biol Chem, 1999. 274(15): p. 10145-53.

110. Chen, R.H., W.J. Wang, and J.C. Kuo, The tumor suppressor DAP-kinase links cell adhesion and cytoskeleton reorganization to cell death regulation. J Biomed Sci, 2006. 13(2): p. 193-9.

111. Ishida, M., et al., The SYT-SSX fusion protein down-regulates the cell proliferation regulator COM1 in $t(x ; 18)$ synovial sarcoma. Mol Cell Biol, 2007. 27(4): p. 1348-55.

112. Xue, D. and H.R. Horvitz, Inhibition of the Caenorhabditis elegans cell-death protease CED-3 by a CED-3 cleavage site in baculovirus p35 protein. Nature, 1995. 377(6546): p. 248-51.

113. Bump, N.J., et al., Inhibition of ICE family proteases by baculovirus antiapoptotic protein p35. Science, 1995. 269(5232): p. 1885-8.

114. Mork, C.N., D.V. Faller, and R.A. Spanjaard, A mechanistic approach to anticancer therapy: targeting the cell cycle with histone deacetylase inhibitors. Curr Pharm Des, 2005. 11(9): p. 1091-104.

115. Shannan, B., et al., Clusterin and DNA repair: a new function in cancer for a key player in apoptosis and cell cycle control. J Mol Histol, 2006. 37(5-7): p. 183-8. 
116. D'Orazi, G., et al., Homeodomain-interacting protein kinase-2 phosphorylates p53 at Ser 46 and mediates apoptosis. Nat Cell Biol, 2002. 4(1): p. 11-9.

117. Sanjo, H., T. Kawai, and S. Akira, DRAKs, novel serine/threonine kinases related to deathassociated protein kinase that trigger apoptosis. J Biol Chem, 1998. 273(44): p. 29066-71.

118. Heddi, A., et al., Coordinate induction of energy gene expression in tissues of mitochondrial disease patients. J Biol Chem, 1999. 274(33): p. 22968-76.

119. Crimi, M., et al., Skeletal muscle gene expression profiling in mitochondrial disorders. Faseb J, 2005. 19(7): p. 866-8.

120. Aure, K., et al., Apoptosis in mitochondrial myopathies is linked to mitochondrial proliferation. Brain, 2006. 129(Pt 5): p. 1249-59.

121. Andersson, U. and R.C. Scarpulla, Pgc-1-related coactivator, a novel, serum-inducible coactivator of nuclear respiratory factor 1-dependent transcription in mammalian cells. Mol Cell Biol, 2001. 21(11): p. 3738-49.

122. Gleyzer, N., K. Vercauteren, and R.C. Scarpulla, Control of mitochondrial transcription specificity factors (TFB1M and TFB2M) by nuclear respiratory factors (NRF-1 and NRF-2) and PGC-1 family coactivators. Mol Cell Biol, 2005. 25(4): p. 1354-66.

123. Park, H., E. Davidson, and M.P. King, The pathogenic A3243G mutation in human mitochondrial tRNALeu(UUR) decreases the efficiency of aminoacylation. Biochemistry, 2003. 42(4): p. 958-64.

124. Moudy, A.M., et al., Abnormal calcium homeostasis and mitochondrial polarization in a human encephalomyopathy. Proc Natl Acad Sci U S A, 1995. 92(3): p. 729-33.

125. Howard, T.L., et al., CHMP1 functions as a member of a newly defined family of vesicle trafficking proteins. J Cell Sci, 2001. 114(Pt 13): p. 2395-404.

126. Tsang, H.T., et al., A systematic analysis of human CHMP protein interactions: additional MIT domain-containing proteins bind to multiple components of the human ESCRT III complex. Genomics, 2006. 88(3): p. 333-46.

127. Bilodeau, P.S., et al., The GAT domains of clathrin-associated GGA proteins have two ubiquitin binding motifs. J Biol Chem, 2004. 279(52): p. 54808-16.

128. Nazarian, J., K. Bouri, and E.P. Hoffman, Intracellular expression profiling by laser capture microdissection: three novel components of the neuromuscular junction. Physiol Genomics, 2005. 21(1): p. 70-80.

129. Zhao, P. and E.P. Hoffman, Embryonic myogenesis pathways in muscle regeneration. Dev Dyn, 2004. 229(2): p. 380-92.

130. Shoubridge, E.A., T. Johns, and G. Karpati, Complete restoration of a wild-type mtDNA genotype in regenerating muscle fibres in a patient with a tRNA point mutation and mitochondrial encephalomyopathy. Hum Mol Genet, 1997. 6(13): p. 2239-42.

131. Taivassalo, T., et al., Aerobic conditioning in patients with mitochondrial myopathies: physiological, biochemical, and genetic effects. Ann Neurol, 2001. 50(2): p. 133-41.

132. Taivassalo, T., et al., Gene shifting: a novel therapy for mitochondrial myopathy. Hum Mol Genet, 1999. 8(6): p. 1047-52.

133. Akagi, M., et al., A point mutation of mitochondrial ATPase 6 gene in Leigh syndrome. Neuromuscul Disord, 2002. 12(1): p. 53-5.

134. Wilson, C.J., et al., Mitochondrial DNA point mutation T9176C in Leigh syndrome. J Child Neurol, 2000. 15(12): p. 830-3.

135. Makino, M., et al., Mitochondrial DNA mutations in Leigh syndrome and their phylogenetic implications. J Hum Genet, 2000. 45(2): p. 69-75.

136. Thyagarajan, D., et al., A novel mitochondrial ATPase 6 point mutation in familial bilateral striatal necrosis. Ann Neurol, 1995. 38(3): p. 468-72.

137. Jacobs, L.J., et al., Transmission and prenatal diagnosis of the T9176C mitochondrial DNA mutation. Mol Hum Reprod, 2005. 11(3): p. 223-8.

138. King, M.P. and G. Attardi, Injection of mitochondria into human cells leads to a rapid replacement of the endogenous mitochondrial DNA. Cell, 1988. 52(6): p. 811-9. 
139. King, M.P. and G. Attardi, Human cells lacking mtDNA: repopulation with exogenous mitochondria by complementation. Science, 1989. 246(4929): p. 500-3.

140. Smyth, G.K., Linear models and empirical bayes methods for assessing differential expression in microarray experiments. Stat Appl Genet Mol Biol, 2004. 3: p. Article3.

141. Smyth, G.K. and T. Speed, Normalization of CDNA microarray data. Methods, 2003. 31(4): p. 265-73.

142. Smyth, G.K., J. Michaud, and H.S. Scott, Use of within-array replicate spots for assessing differential expression in microarray experiments. Bioinformatics, 2005. 21(9): p. 2067-75.

143. Benjamini, Y. and Y. Hochberg, Controlling the false discovery rate: a practical andpowerful approach to multiple testing. J. R. Statist. Soc. B, 1995. 57: p. 289-300.

144. Jacobs, J.A.M., The transmission and seggregation of mitochondrial DNA mutations. Department of Genetics and Cell Biology, Research institute GROW (Growth and development). Maastricht University. Maastricht, 2007.

145. Lademann, U., T. Kallunki, and M. Jaattela, A20 zinc finger protein inhibits TNF-induced apoptosis and stress response early in the signaling cascades and independently of binding to TRAF2 or 14-3-3 proteins. Cell Death Differ, 2001. 8(3): p. 265-72.

146. Liu, Z.J., X. Lu, and S. Zhong, ASPP--Apoptotic specific regulator of p53. Biochim Biophys Acta, 2005. 1756(1): p. 77-80.

147. Deveraux, Q.L., et al., IAPs block apoptotic events induced by caspase-8 and cytochrome $c$ by direct inhibition of distinct caspases. Embo J, 1998. 17(8): p. 2215-23.

148. Kobayashi, S., et al., 53BP2 induces apoptosis through the mitochondrial death pathway. Genes Cells, 2005. 10(3): p. 253-60.

149. Sunayama, J., et al., Physical and functional interaction between BHz-only protein Hrk and mitochondrial pore-forming protein p32. Cell Death Differ, 2004. 11(7): p. 771-81.

150. Brady, S.C., L.A. Allan, and P.R. Clarke, Regulation of caspase 9 through phosphorylation by protein kinase C zeta in response to hyperosmotic stress. Mol Cell Biol, 2005. 25(23): p. 10543-55.

151. Paschal, B.M., R.A. Obar, and R.B. Vallee, Interaction of brain cytoplasmic dynein and MAP2 with a common sequence at the $C$ terminus of tubulin. Nature, 1989. 342(6249): p. 569-72.

152. Oh, S.W., et al., Archvillin, a muscle-specific isoform of supervillin, is an early expressed component of the costameric membrane skeleton. J Cell Sci, 2003. 116(Pt 11): p. 2261-75.

153. Harris, A.S. and J.S. Morrow, Calmodulin and calcium-dependent protease I coordinately regulate the interaction of fodrin with actin. Proc Natl Acad Sci U S A, 1990. 87(8): p. 300913.

154. Canizalez-Roman, A. and F. Navarro-Garcia, Fodrin CaM-binding domain cleavage by Pet from enteroaggregative Escherichia coli leads to actin cytoskeletal disruption. Mol Microbiol, 2003. 48(4): p. 947-58.

155. Alcivar, A., et al., DEDD and DEDD2 associate with caspase-8/10 and signal cell death. Oncogene, 2003. 22(2): p. 291-7.

156. Zhang, H., et al., Involvement of programmed cell death 4 in transforming growth factorbeta1-induced apoptosis in human hepatocellular carcinoma. Oncogene, 2006. 25(45): p. 6101-12.

157. Takahashi, Y., et al., Loss of Bif-1 suppresses Bax/Bak conformational change and mitochondrial apoptosis. Mol Cell Biol, 2005. 25(21): p. 9369-82.

158. Hasegawa, M., et al., Mechanism of ASC-mediated apoptosis: bid-dependent apoptosis in type Il cells. Oncogene, 2007. 26(12): p. 1748-56.

159. Lee, J.H., S.B. Rho, and T. Chun, Programmed cell death 6 (PDCD6) protein interacts with death-associated protein kinase 1 (DAPk1): additive effect on apoptosis via caspase-3 dependent pathway. Biotechnol Lett, 2005. 27(14): p. 1011-5.

160. Vito, P., E. Lacana, and L. D'Adamio, Interfering with apoptosis: $\mathrm{Ca}(2+)$-binding protein ALG2 and Alzheimer's disease gene ALG-3. Science, 1996. 271(5248): p. 521-5. 
161. Chen, M.C., et al., The role of apoptosis signal-regulating kinase 1 in lymphotoxin-beta receptor-mediated cell death. J Biol Chem, 2003. 278(18): p. 16073-81.

162. Nakano, H., et al., TRAF5, an activator of NF-kappaB and putative signal transducer for the lymphotoxin-beta receptor. J Biol Chem, 1996. 271(25): p. 14661-4.

163. Kuai, J., et al., Endogenous association of TRAF2, TRAF3, CIAP1, and Smac with lymphotoxin beta receptor reveals a novel mechanism of apoptosis. J Biol Chem, 2003. 278(16): p. 143639.

164. Chatellard-Causse, C., et al., Alix (ALG-2-interacting protein X), a protein involved in apoptosis, binds to endophilins and induces cytoplasmic vacuolization. J Biol Chem, 2002. 277(32): p. 29108-15.

165. Gomez, D.E., et al., Tissue inhibitors of metalloproteinases: structure, regulation and biological functions. Eur J Cell Biol, 1997. 74(2): p. 111-22.

166. Silacci, P., et al., Gelsolin superfamily proteins: key regulators of cellular functions. Cell Mol Life Sci, 2004. 61(19-20): p. 2614-23.

167. Klampfer, L., et al., Oncogenic Ras promotes butyrate-induced apoptosis through inhibition of gelsolin expression. J Biol Chem, 2004. 279(35): p. 36680-8.

168. Ahn, J.S., et al., Gelsolin for senescence-associated resistance to apoptosis. Ann N Y Acad Sci, 2003. 1010: p. 493-5.

169. Tondera, D., et al., Knockdown of MTP18, a novel phosphatidylinositol 3-kinase-dependent protein, affects mitochondrial morphology and induces apoptosis. J Biol Chem, 2004. 279(30): p. 31544-55.

170. Wang, K.K., et al., Simultaneous degradation of alphall- and betall-spectrin by caspase 3 (CPP32) in apoptotic cells. J Biol Chem, 1998. 273(35): p. $22490-7$.

171. Tang, H.L., A.H. Le, and H.L. Lung, The increase in mitochondrial association with actin precedes Bax translocation in apoptosis. Biochem J, 2006. 396(1): p. 1-5.

172. Mirabella, M., et al., Apoptosis in mitochondrial encephalomyopathies with mitochondrial DNA mutations: a potential pathogenic mechanism. Brain, 2000.123 ( Pt 1): p. 93-104.

173. Shidara, Y., et al., Positive contribution of pathogenic mutations in the mitochondrial genome to the promotion of cancer by prevention from apoptosis. Cancer Res, 2005. 65(5): p. $1655-63$.

174. Geromel, V., et al., Superoxide-induced massive apoptosis in cultured skin fibroblasts harboring the neurogenic ataxia retinitis pigmentosa (NARP) mutation in the ATPase- 6 gene of the mitochondrial DNA. Hum Mol Genet, 2001. 10(11): p. 1221-8.

175. Kwong, J.Q., et al., The mitochondrial respiratory chain is a modulator of apoptosis. J Cell Biol, 2007. 179(6): p. 1163-77.

176. Ostergaard, E., et al., Hypertrichosis in patients with SURF1 mutations. Am J Med Genet A, 2005. 138(4): p. 384-8.

177. Rahman, S., et al., A SURF1 gene mutation presenting as isolated leukodystrophy. Ann Neurol, 2001. 49(6): p. 797-800.

178. Salviati, L., et al., Novel SURF1 mutation in a child with subacute encephalopathy and without the radiological features of Leigh Syndrome. Am J Med Genet A, 2004. 128(2): p. 195-8.

179. Tiranti, V., et al., Leigh syndrome transmitted by uniparental disomy of chromosome 9. J Med Genet, 1999. 36(12): p. 927-8.

180. Riordan-Eva, P. and A.E. Harding, Leber's hereditary optic neuropathy: the clinical relevance of different mitochondrial DNA mutations. J Med Genet, 1995. 32(2): p. 81-7.

181. Bodnar, A.G., et al., Nuclear complementation restores mtDNA levels in cultured cells from a patient with mtDNA depletion. Am J Hum Genet, 1993. 53(3): p. 663-9.

182. Ugalde, C., et al., Differences in assembly or stability of complex I and other mitochondrial OXPHOS complexes in inherited complex I deficiency. Hum Mol Genet, 2004. 13(6): p. 65967. 
183. Scacco, S., et al., Pathological mutations of the human NDUFS4 gene of the 18-kDa (AQDQ) subunit of complex I affect the expression of the protein and the assembly and function of the complex. J Biol Chem, 2003. 278(45): p. 44161-7.

184. van der Westhuizen, F.H., et al., Human mitochondrial complex I deficiency: investigating transcriptional responses by microarray. Neuropediatrics, 2003. 34(1): p. 14-22.

185. Pandya, G.A., et al., A bioinformatic filter for improved base-call accuracy and polymorphism detection using the Affymetrix GeneChip whole-genome resequencing platform. Nucleic Acids Res, 2007. 35(21): p. e148.

186. Wong, L.J., Pathogenic mitochondrial DNA mutations in protein-coding genes. Muscle Nerve, 2007.36(3): p. 279-93.

187. Valentino, M.L., et al., The $13042 \mathrm{G}-->$ A/ND5 mutation in mtDNA is pathogenic and can be associated also with a prevalent ocular phenotype. J Med Genet, 2006. 43(7): p. e38.

188. Liu, C., et al., Novel resequencing chip customized to diagnose mutations in patients with inherited syndromes of intrahepatic cholestasis. Gastroenterology, 2007. 132(1): p. 119-26.

189. Albert, T.J., et al., Direct selection of human genomic loci by microarray hybridization. Nat Methods, 2007. 4(11): p. 903-5.

190. Hodges, E., et al., Genome-wide in situ exon capture for selective resequencing. Nat Genet, 2007.

191. Okou, D.T., et al., Microarray-based genomic selection for high-throughput resequencing. Nat Methods, 2007. 4(11): p. 907-9.

192. Porreca, G.J., et al., Multiplex amplification of large sets of human exons. Nat Methods, 2007. 4(11): p. 931-6.

193. Wang, C., et al., Characterization of mutation spectra with ultra-deep pyrosequencing: application to HIV-1 drug resistance. Genome Res, 2007. 17(8): p. 1195-201.

194. Hoffmann, C., et al., DNA bar coding and pyrosequencing to identify rare HIV drug resistance mutations. Nucleic Acids Res, 2007.35(13): p. e91.

195. Olson, M., Enrichment of super-sized resequencing targets from the human genome. Nat Methods, 2007. 4(11): p. 891-2.

196. Wittwer, C.T., et al., High-resolution genotyping by amplicon melting analysis using LCGreen. Clin Chem, 2003. 49(6 Pt 1): p. 853-60.

197. Liew, M., et al., Genotyping of single-nucleotide polymorphisms by high-resolution melting of small amplicons. Clin Chem, 2004. 50(7): p. 1156-64.

198. Reed, G.H. and C.T. Wittwer, Sensitivity and specificity of single-nucleotide polymorphism scanning by high-resolution melting analysis. Clin Chem, 2004. 50(10): p. 1748-54.

199. Palais, R.A., M.A. Liew, and C.T. Wittwer, Quantitative heteroduplex analysis for single nucleotide polymorphism genotyping. Anal Biochem, 2005. 346(1): p. 167-75.

200. Bannwarth, S., V. Procaccio, and V. Paquis-Flucklinger, Surveyor Nuclease: a new strategy for a rapid identification of heteroplasmic mitochondrial DNA mutations in patients with respiratory chain defects. Hum Mutat, 2005. 25(6): p. 575-82.

201. Bannwarth, S., V. Procaccio, and V. Paquis-Flucklinger, Rapid identification of unknown heteroplasmic mutations across the entire human mitochondrial genome with mismatchspecific Surveyor Nuclease. Nat Protoc, 2006. 1(4): p. 2037-47.

202. Mashkevich, G., et al., SHY1, the yeast homolog of the mammalian SURF-1 gene, encodes a mitochondrial protein required for respiration. J Biol Chem, 1997. 272(22): p. 14356-64.

203. Nijtmans, L.G., et al., Shy1p occurs in a high molecular weight complex and is required for efficient assembly of cytochrome c oxidase in yeast. FEBS Lett, 2001. 498(1): p. 46-51.

204. Barrientos, A., D. Korr, and A. Tzagoloff, Shyp is necessary for full expression of mitochondrial COX1 in the yeast model of Leigh's syndrome. Embo J, 2002. 21(1-2): p. 43-52.

205. Barrientos, A., A. Zambrano, and A. Tzagoloff, Mss51p and Cox14p jointly regulate mitochondrial Coxip expression in Saccharomyces cerevisiae. Embo J, 2004. 23(17): p. 347282. 
206. Mick, D.U., et al., Shy1 couples Cox1 translational regulation to cytochrome c oxidase assembly. Embo J, 2007. 26(20): p. 4347-58.

207. Agostino, A., et al., Constitutive knockout of Surf1 is associated with high embryonic lethality, mitochondrial disease and cytochrome $c$ oxidase deficiency in mice. Hum Mol Genet, 2003. 12(4): p. 399-413.

208. Wallace, D.C., Mouse models for mitochondrial disease. Am J Med Genet, 2001. 106(1): p. 7193.

209. Wallace, D.C., Animal models for mitochondrial disease. Methods Mol Biol, 2002. 197: p. 354.

210. Poulton, J., et al., Deficiency of the human mitochondrial transcription factor h-mLTFA in infantile mitochondrial myopathy is associated with mtDNA depletion. Hum Mol Genet, 1994. 3(10): p. 1763-9.

211. Graham, B.H., et al., A mouse model for mitochondrial myopathy and cardiomyopathy resulting from a deficiency in the heart/muscle isoform of the adenine nucleotide translocator. Nat Genet, 1997. 16(3): p. 226-34.

212. Zhang, D., et al., Mitochondrial DNA mutations activate the mitochondrial apoptotic pathway and cause dilated cardiomyopathy. Cardiovasc Res, 2003 . 57(1): p. 147-57.

213. Zhang, D., et al., Construction of transgenic mice with tissue-specific acceleration of mitochondrial DNA mutagenesis. Genomics, 2000. 69(2): p. 151-61.

214. Kasahara, T., et al., Mice with neuron-specific accumulation of mitochondrial DNA mutations show mood disorder-like phenotypes. Mol Psychiatry, 2006. 11(6): p. 577-93, 523.

215. Sligh, J.E., et al., Maternal germ-line transmission of mutant mtDNAs from embryonic stem cell-derived chimeric mice. Proc Natl Acad Sci U S A, 2000. 97(26): p. 14461-6.

216. Inoue, K., et al., Generation of mice with mitochondrial dysfunction by introducing mouse mtDNA carrying a deletion into zygotes. Nat Genet, 2000. 26(2): p. 176-81.

217. Sanchez-Martinez, A., et al., Modeling human mitochondrial diseases in flies. Biochim Biophys Acta, 2006. 1757(9-10): p. 1190-8.

218. Zordan, M.A., et al., Post-transcriptional silencing and functional characterization of the Drosophila melanogaster homolog of human Surf1. Genetics, 2006. 172(1): p. 229-41.

219. Tsang, W.Y., et al., Mitochondrial respiratory chain deficiency in Caenorhabditis elegans results in developmental arrest and increased life span. J Biol Chem, 2001. 276(34): p. 322406.

220. Benit, P., et al., Large-scale deletion and point mutations of the nuclear NDUFV1 and NDUFS1 genes in mitochondrial complex I deficiency. Am J Hum Genet, 2001. 68(6): p. 1344-52.

221. Schuelke, M., et al., Mutant NDUFV1 subunit of mitochondrial complex 1 causes leukodystrophy and myoclonic epilepsy. Nat Genet, 1999. 21(3): p. 260-1.

222. Senoo-Matsuda, N., et al., A defect in the cytochrome $b$ large subunit in complex II causes both superoxide anion overproduction and abnormal energy metabolism in Caenorhabditis elegans. J Biol Chem, 2001. 276(45): p. 41553-8.

223. Ma, Y.S., et al., Upregulation of matrix metalloproteinase 1 and disruption of mitochondrial network in skin fibroblasts of patients with MERRF syndrome. Ann N Y Acad Sci, 2005. 1042: p. 55-63.

224. Danielson, S.R., et al., Isolation of transcriptomal changes attributable to LHON mutations and the cybridization process. Brain, 2005. 128(Pt 5): p. 1026-37.

225. Jahangir Tafrechi, R.S., et al., Distinct nuclear gene expression profiles in cells with mtDNA depletion and homoplasmic A3243G mutation. Mutat Res, 2005. 578(1-2): p. 43-52.

226. Cortopassi, G., et al., Mitochondrial disease activates transcripts of the unfolded protein response and cell cycle and inhibits vesicular secretion and oligodendrocyte-specific transcripts. Mitochondrion, 2006. 6(4): p. 161-75.

227. Gerard, B., et al., Uridine preserves the expression of respiratory enzyme deficiencies in cultured fibroblasts. Eur J Pediatr, 1993. 152(3): p. 270. 
228. Saitoh, S., et al., Single-cell analysis of mitochondrial DNA in patients and a carrier of the tRNA(Leu)(UUR) gene mutation. J Inherit Metab Dis, 1999. 22(5): p. 608-14.

229. Geromel, V., et al., Coenzyme Q10 depletion is comparatively less detrimental to human cultured skin fibroblasts than respiratory chain complex deficiencies. Free Radic Res, 2002. 36(4): p. 375-9. 


\section{Summary}

One of the most important functions of mitochondria is the production of energy through the process of oxidative phosphorylation (OXPHOS). The first part of OXPHOS is carried out by the electron transport chain, consisting of OXPHOS enzyme complexes I to IV. In the final step, which is catalysed by the fifth OXPHOS enzyme complex, ATP synthase or complex $V$, energy is generated in the form of ATP. OXPHOS is under dual genetic control of the nuclear DNA (nDNA) and the mitochondrial DNA (mtDNA). The mtDNA encodes 13 subunits of the OXPHOS enzyme complexes, 22 tRNA and two rRNA molecules. The vast majority of the subunits and all other mitochondrial proteins, involved in mtDNA transcription, translation, replication, or in mitochondrial transport, assembly and biogenesis, are encoded by nuclear genes. Mutations in the mtDNA are either de novo, segregate in the maternal lineage or are somatically acquired. MtDNA mutations can be either heteroplasmic (mutant and wild-type mtDNA molecules cosegregate within cells and tissues) or homoplasmic (all mtDNA molecules are mutated). Mutations in mtDNA genes and in nuclear OXPHOS genes can lead to OXPHOS deficiency and energy deficit. Disorders in which OXPHOS deficiency is a central characteristic, are referred to as oxidative phosphorylation or OXPHOS disorders.

OXPHOS disorders are clinically and genetically heterogeneous. This means that the same mutation can lead to different symptoms and that different mutations can lead to the same symptoms respectively. This makes it difficult to determine the genetic defect and to explain the disease pathology. Clinical heterogeneity is particularly an issue for mtDNA mutations, which can only partly be explained by different levels of heteroplasmy. Other factors must play a role as well. Symptom-based protocols have been explored to link clinical symptoms directly to a specific mtDNA mutation, but the suitability is limited to certain specific syndromes, as for example with Leber hereditary optic atrophy (LHON). Alternatively the entire mtDNA could be screened to identify a genetic defect or to exclude the mtDNA as a causative factor, which is important for genetic counseling. For example, mtDNA mutations are causative in $25 \%$ of the pediatric OXPHOS disorder cases, making it is reasonable to screen the complete mtDNA. In chapter $\mathbf{2}$ a microarray based method is applied to resequence the complete mtDNA in OXPHOS patients. Call rates were high ( $94 \%$ on average) and heteroplasmic mutations were detectable at levels above $5 \%$, although the lower limits were not systematically evaluated. In a quarter of the OXPHOS patients a genetic diagnosis could be made, but one has to be aware that unclassified variants are frequently detected, which cause interpretation problems and, if not properly counseled, anxiety in the families involved. Optimisation of data analysis and software tools will improve call rates and heteroplasmy level detection limits comparable to the currently used heteroduplex based methods. Databases and functional assays will need to solve the interpretation problems, which is common to all large-scale sequencing approaches. A first step will be central storage of all detected nucleotide variants and the development of standardised diagnostic flow charts in 
which as many aspects as possible of a pathogenicity scoring mechanisms are taken into account. The improvements in data analysis and the costs of the chip compared with new high throughput sequencing and mutation detection techniques will determine whether MitoChip resequencing remains the preferred method for mtDNA resequencing for the next couple of years.

Microarray gene expression profiling has been shown to be extremely powerful to study pathological molecular processes of diseases. This approach was applied to OXPHOS disorders on skeletal muscle from Leigh syndrome patients with a mutation in the nuclear SURF1 gene, which is fatal at infancy (chapter 3 ) and from symptomatic and a-symptomatic carriers of the m.3243A $>C$ mutation in the mtDNA, which can cause a variety of symptoms, including mitochondrial myopathy, encephalopathy, lactic acidosis, and stroke-like episodes (MELAS, chapter 4). Our data show that stimulation of OXPHOS and mitochondrial biogenesis does not occur to compensate the energy deficiency in symptomatic SURF1 Leigh and MELAS patients. The number of altered processes was fairly limited, but included an unexpected elevation of the complement system. In a-symptomatic carriers of the m.3243A $>\mathrm{G}$ mutation many more processes were altered, including protein turn-over, apoptosis and also, at a lower level, the complement system. Protein studies showed that ROS-damage was elevated in SURF1- and MELAS-muscle biopsies. Based on our data, we hypothesise that increased ROS production, resulting from a defective OXPHOS system and a defect in the translation machinery in case of the m.3243A>C mutation, results in oxidative damaged and dysfunctional proteins. In an effort to replace the damaged and dysfunctional proteins, protein turnover and apoptosis is increased. However, as dysfunctional proteins are constantly produced, this can not prevent, but only delay pathology from occurring, probably depending on the energy capacity of the tissue. When damage is insufficiently removed, irreparable pathology arises. Compensation and rescue mechanisms are shut-down and, as a final rescue process, complement components are strongly activated as stimulators of muscle regeneration. As the genetic defect is present in all cells this final effort is doomed to fail.

Gene expression studies on OXPHOS disorders are limited by the rareness of specific syndromes and the scarcity of available tissue. Alternative animal models not always represent the wild-type or pathological situation in human to the best extent, making extrapolation of the results difficult. Therefore, patient-derived model systems should be better alternatives. In chapter $\mathbf{5}$, a cell culture model system is presented which exploits the mutation load differences between monoclonal fibroblast cultures. This circumvents the main problems of other cell culture model systems, i.e. differences in genetic backgrounds between individuals or genetically modified and unstable genetic backgrounds in transformed cell lines. Fibroblast cells from one single patient carrying the m.9176T $>\mathrm{C}$ mutation in the ATP6 gene were cloned by seeding the cells in a very low concentration, allowing cell colonies to arise from only one single cell. This gave rise to various cell lines with the same genetic background, but with different m.9176T>C mutation loads. This system is the preferred method to study mutation load effects of the m.9176T>C mutation. Moreover, this cell culture model system may be applicable to other mtDNA mutations as well, 
like the MELAS m.3243A>G and the Neuropathy, Ataxia, and Retinitis Pigmentosa (NARP) or Leigh syndrome m.8993T $>\mathrm{G} / \mathrm{C}$ mutation.

In conclusion, chip-based approaches have been shown to be valuable tools for studying OXPHOS diseases. Classification based on gene expression profiling or other clinical or biochemical criteria remains difficult and only applicable to a small subgroup of patients, due to the extreme genetic and clinical heterogeneity of these disorders. Therefore, high-throughput screening methods, like the mtDNA resequencing chips, have been developed as an alternative to rapidly screen large amounts of genes, if no educated choice can be made in advance. Gene expression profiling is particularly suited to identify the pathological molecular genetic pathways, involved in OXPHOS disease. Apparently, the OXPHOS system itself reacts only limitedly at the transcriptional level and for the m.3243A>C mutation only before symptoms occur. Other processes involved are ROS damage, protein turnover and tissue regeneration, which may provide new clues for the development of therapies for OXPHOS disorders. Increasing energy capacity may delay pathology. Preventing ROS damage to proteins, DNA and tissue, by preventing excessive ROS production, but also by improving ROS protection, seems to be beneficial. Finally, stimulating protein turnover and supplying the required energy capacity might be helpful in order to compensate for the defects and postpone disease manifestation. Further studies will have to be performed to demonstrate the efficacy of these approaches. 


\section{Samenvatting}

Een van de meest belangrijkste functies van mitochondria is de productie van energie via het proces van oxidatieve fosforylering (OXPHOS). Het eerste deel van OXPHOS wordt gevormd door de electronentransportketen die bestaat uit de OXPHOS enzymcomplexen I tot en met IV. In de laatste stap, die gekatalyseerd wordt door het vijfde OXPHOS enzymcomplex, ATP synthase of complex V, wordt energie gegenereerd in de vorm van ATP. OXPHOS is zowel onder controle van het nucleaire DNA (nDNA) als het mitochondriële DNA (mtDNA). Het mtDNA codeert voor 13 subunits van het OXPHOS enzymcomplex, 22 tRNA en twee rRNA moleculen. De meerderheid van de subunits en alle andere mitochondriële eiwitten, betrokken bij mtDNA transcriptie, translatie, replicatie, of bij mitochondrieel transport, opbouw en biogenese, worden gecodeerd door nucleaire genen. Mutaties in het mtDNA kunnen de novo zijn, worden overgedragen via de maternale lijn of worden somatisch verworven. MtDNA mutaties kunnen heteroplasmisch (mutante en wild-type mtDNA moleculen zijn samen aanwezig in cellen en weefsels) of homoplasmisch (alle mtDNA moleculen zijn gemuteerd) zijn. Mutaties in mtDNA genen en in nucleaire OXPHOS genen kunnen leiden tot OXPHOS deficiëntie en een energietekort. Ziektes waarbij OXPHOS deficiëntie een centrale rol speelt worden oxidatieve fosforyleringsstoornissen of OXPHOS stoornissen genoemd.

OXPHOS stoornissen zijn klinisch en genetisch heterogeen. Dit betekent respectievelijk dat dezelfde mutatie kan leiden tot verschillende symptomen, en dat verschillende mutaties kunnen leiden tot dezelfde symptomen. Dit bemoeilijkt het bepalen van het genetisch defect en het verklaren van de pathologie achter de ziekte. Klinische heterogeniteit is vooral een knelpunt in het geval van mtDNA mutaties, wat alleen gedeeltelijk verklaard kan worden door verschillen in heteroplasmieniveaus. Andere factoren zullen ook een rol spelen. Er zijn symptoomgebaseerde protocollen ontwikkeld die trachten een directe link tussen de klinische symptomen en een mtDNA mutatie te leggen, maar de bruikbaarheid hiervan blijft beperkt tot bepaalde specifieke syndromen, zoals Leber hereditary optic atrophy (LHON). Als alternatief zou het volledige mtDNA gescreend kunnen worden om het genetisch defect te identificeren of om het mtDNA als oorzakelijke factor uit te sluiten, wat belangrijk is voor de genetische counseling. MtDNA mutaties zijn bijvoorbeeld de ziekteoorzaak in 25\% van de pediatrische OXPHOS stoornis gevallen, dat het nut van het screenen van het gehele mtDNA benadrukt. In hoofdstuk 2 wordt een op microarray gebaseerde techniek gebruikt om het gehele mtDNA van patiënten met een OXPHOS stoornis te resequencen. Callrates waren hoog (gemiddeld 94\%) en heteroplasmische mutaties waren detecteerbaar tot niveaus boven de $5 \%$, hoewel de lagere limiet niet systematisch geëvalueerd is. In een kwart van de OXPHOS patiënten kon een genetische diagnose worden gesteld, maar er moet niet vergeten worden dat er vaak ongeclassificeerde varianten worden gedetecteerd, die de interpretatie kunnen bemoeilijken en die, wanneer niet goed gecounseld, angst in de betrokken families kan veroorzaken. Optimalisatie van de data analyse en software tools zal de callrates en 
de heterplasmiedetectielimiet doen verbeteren tot een vergelijkbaar niveau als de huidige op heteroduplex gebaseerde methodes. Databases en functionele tests zullen het interpretatieprobleem dat voorkomt bij alle grootformaat sequencing aanpakken moeten verhelpen. De centrale opslag van alle gedetecteerd nucleotidevarianten, en de ontwikkeling van gestandaardiseerde diagnostische werkschema's waarin zo veel mogelijk aspecten van een pathogeniciteitsscroringsmechanisme worden aangehaald, zal een eerste stap vormen. De verbeteringen in de data analyse en de kosten van de chip in vergelijking met nieuwe high throughput sequencing- en mutatiedetectietechnieken zullen bepalend zijn voor de vraag of MitoChip resequencing de voorkeursmethode zal blijven voor het resequencen van het mtDNA in de komende jaren.

Het is gebleken dat microarray genexpressie profilering zeer waardevol is voor het bestuderen van pathologisch moleculaire processen van ziektes. Deze aanpak werd toegepast op skeletspier van patiënten met een OXPHOS stoornis zoals Leigh syndroom patiënten met een mutatie in het nucleaire SURF1 gen die fataal is tijdens de kinderjaren (hoofdstuk 3) en symptomatische en asymptomatische dragers van de m.3243A $>G$ mutatie in het mtDNA die kan resulteren in verschillende symptomen, waaronder mitochondrial myopathy, encephalopathy, lactic acidosis, and stroke-like episodes (MELAS, hoofdstuk 4). Onze data illustreert dat stimulatie van OXPHOS en mitochondriële biogenese niet optreedt ter compensatie van het energietekort in symptomatische SURF1 Leigh en MELAS patiënten. Het aantal veranderde processen was aan de lage kant, maar bevatte een onverwachte stimulatie van het complement systeem. In a-symptomatische dragers van de m.3243A>G mutatie waren veel meer processen veranderd, waaronder eiwitturnover, apoptose en ook, weliswaar minder sterk, het complement systeem. Eiwitstudies hebben aangetoond dat de mate van ROS-schade verhoogd was in SURF1 en MELAS spierbiopten. Op basis van onze data stellen wij de hypothese dat de toename in ROS-productie, veroorzaakt door het defecte OXPHOS systeem en een defect in het translatieapparaat bij de m.3243A>C mutatie, leidt tot oxidatief beschadigde en disfunctionele eiwitten. Omdat disfunctionele eiwitten constant geproduceerd worden, kan dit mechanisme het ontstaan van de pathologie niet voorkomen, maar alleen vertragen, wat afhankelijk is van de energiecapaciteit van het weefsel. Wanneer de schade inefficiënt verwijderd wordt, ontstaat er een onomkeerbare pathologie. Compensatie- en reddingsmechanismen worden gestopt en complement componenten worden sterk geactiveerd voor de stimulatie van spierregeneratie als een laatste redmiddel. Omdat het genetisch defect in alle cellen aanwezig is, is deze laatste poging gedoemd om te mislukken.

De aanwezigheid van genexpressiestudies op OXPHOS stoornissen is beperkt door de zeldzaamheid van specifieke syndromen en de schaarsheid aan beschikbaar weefsel. Alternatieve diermodellen representeren de wild-type of de pathologische situatie in de mens niet altijd even goed, wat extrapolatie van de resultaten bemoeilijkt. Daarom zouden van de patiënt afgeleide modelsystemen betere alternatieven zijn. In hoofdstuk 5 wordt een celkweekmodelsysteem 
beschreven dat gebruik maakt van de verschillen in mutatiepercentage tussen monoklonale fibroblastenkweken. Dit omzeilt de belangrijkste problemen van andere celkweekmodelsystemen, namelijk de verschillen in genetische achtergronden tussen individuen of genetische gemodificeerde en onstabiele genetische achtergronden in getransformeerde cellijnen. Fibroblastcellen van één patiënt die drager was van de m.9176T >C mutatie in het ATP6 gen werden gekloneerd door de cellen uit te zaaien in een zeer lage concentratie waardoor celkolonies konden ontstaan vanuit één enkele cel. Hierdoor ontstonden verschillende cellijnen met dezelfde genetische achtergrond, maar met verschillende m.9176T $>C$ mutatiepercentages. Dit systeem is de voorkeursmethode voor het bestuderen van mutatiepercentage effecten van de m.9176T>C mutatie. Daarnaast zou dit celkweekmodelsysteem ook toepasbaar zijn op andere mtDNA mutaties, zoals de MELAS m.3243A>G en de Neuropathy, Ataxia, and Retinitis Pigmentosa (NARP) of Leigh syndrome m.8993T>G/C mutatie.

Concluderend kunnen we stellen dat de op chip-gebaseerde aanpakken waardevol zijn gebleken voor de bestudering van OXPHOS stoornissen. Classificatie op basis van genexpressieprofilering of andere klinische of biochemische criteria blijft lastig vanwege de extreme genetische en klinische heterogeniteit van deze stoornissen, en alleen toepasbaar op een kleine subgroep patiënten. Daarom zijn alternatieve high-throughput screeningsmethodes ontwikkeld, zoals de mtDNA resequencing chip, als een alternatief om snel een grote hoeveelheid genen te screenen wanneer geen weloverwogen gerichte keuze op voorhand gemaakt kan worden. Genexpressieprofilering is vooral geschikt voor het identificeren van de pathologische moleculaire genetische pathways die betrokken zijn bij OXPHOS stoornissen. Blijkbaar is er maar een beperkte reactie van het OXPHOS systeem zelf op het transcriptionele niveau, en bij de m.3243A>G mutatie alleen vóórdat symptomen optreden. Andere processen die een rol spelen zijn ROS schade, eiwit turnover en weefselregeneratie, welke nieuwe ingangen kunnen vormen voor de ontwikkeling van therapieën voor OXPHOS stoornissen. Het doen toenemen van de energiecapaciteit zou het optreden van pathologische verschijnselen kunnen vertragen. Het voorkomen van ROS-schade aan eiwitten, DNA en weefsel, door het voorkomen van buitensporige ROS-productie, maar ook door het verbeteren van de ROS-bescherming, zal een positieve invloed hebben. Als laatste zou het stimuleren van de eiwitturnover en het aanreiken van de benodigde energie hiervoor helpen bij de compensatie van het defect, waardoor het optreden van ziektesymptomen uitgesteld wordt. Er zal aanvullend onderzoek uitgevoerd moeten worden om de doeltreffendheid van deze aanpakken vast te stellen. 


\section{Dankwoord}

Ik kan bijna niet geloven dat het eindelijk zover is. Het eindproduct van een aantal jaren hard werken is er eindelijk. Deze periode had zowel ups als downs, maar beide hebben ervoor gezorgd dat ik gegroeid ben als mens en als wetenschappelijk onderzoeker en hebben daarmee bijgedragen aan de totstandkoming van dit proefschrift. Dit alles heb ik natuurlijk niet alleen aan mezelf te danken. Ook de hulp en steun van anderen is in dit traject van groot belang geweest.

Als eerste wil ik mijn promotor en copromotor bedanken. Joep, jij was een promotor in de vorm van een "stille vennoot", vooral in de eerste jaren. Een stille vennoot houdt de gang van zaken van een afstandje in de gaten. Je was er dan ook wanneer je er moest zijn, met name in de eindfase. Beste Joep, bedankt voor je bijdrage. Bert, jou wil ik vooral bedanken voor je steun en begrip in moeilijke tijden, vooral ook tijdens moeilijke tijden buiten het werkvlak. Mede dankzij deze steun en hulp, heb ik dit traject met succes kunnen afronden. Wat het onderzoek betreft lagen we niet altijd op één lijn, wat soms voor stevige discussies zorgde. Ook dat heeft eraan bijgedragen dat ik in wetenschappelijk opzicht gegroeid ben.

Natuurlijk verdienen mijn directe collega's een dankjewel. Ik wil beginnen met de harde AIO-kern, die in het begin gevormd werd door Lorraine, Bianca en Lars, en later werd uitgebreid met Nicole, Florence, Ruben en An. Lorraine, ons raakvlak op onderzoeksgebied was de m.9176T>C mutatie, waarvoor jij een PGD ging opzetten. Van jouw vooronderzoek heb ik dankbaar gebruik gemaakt bij het opzetten van mijn PCR's en mutatiepercentage analyses. Bedankt voor het delen van je expertise op dit gebied. Jij was ook altijd van de partij als er gestapt moest worden. Herinner je je nog die grote neger in het Haagse café? Hoewel hij Frans sprak, wist hij je duidelijk te maken dat hij je wel zag zitten. Jammer genoeg (voor hem) was dat niet wederzijds. Bianca, wij kennen elkaar al veel langer. We zijn in hetzelfde jaar (in 1995 - zijn we al zo oud?!) aan de studie Gezondheidswetenschappen begonnen, maar na een jaar of 2-3 werd ons contact wat minder. Het was voor jou dan ook een verassing dat ik in 2002 bij PopGen/KlinGen begon als AIO. Op mijn eerste werkdag was Bert er niet, en jij moest mij maar even opvangen. Dat was een beetje onwennig, maar het was fijn om een bekend gezicht te zien. Dat onwennige ging er gelukkig weer snel af. Hoewel jouw onderzoeksonderwerp met de muizen wat verder van mijn onderwerp af stond, heb ik toch veel aan jou gehad. Je hebt me een beetje wegwijs gemaakt in het AIO zijn en de gang van zaken geïntroduceerd op de afdeling Popgen. Verder heb je mijn woordenschat uit weten te breiden. Dankzij jou weet ik nu wat "poepig" is. Ik betrap mezelf erop dat ik het woord ook soms gebruik. Lars en Nicole, naast mijn AIO collega's ook mijn paranimfen, ik wil jullie natuurlijk bedanken voor jullie hulp en steun tijdens het hele traject. Zowel op het werkvlak en privé heb ik veel steun aan jullie gehad. Ook al had ons onderzoek soms weinig raakvlakken, doordat we op dezelfde afdeling werkten hadden we toch dingen gemeen waardoor we toch veel steun aan elkaar hebben gehad. Lars, het afgelopen (bijna) anderhalf jaar heb ik door mijn 
“dubbele baan" (mijn werk in Leuven en daarnaast het schrijven van mijn proefschrift) een beetje jouw slaappatroon overgenomen. Maar ik ben blij dat ik ervan af kan stappen; 4-5 uurtjes slaap per dag is toch wat weinig, ik heb er toch wel 6 nodig. Van jou heb ik ook veel geleerd over het analyseren van microarray data. Het programmeren in $\mathrm{R}$ was voor jou als spreken in een tweede moedertaal, en mede dankzij jou spreek ik deze taal nu ook redelijk vloeiend. Nicole, wat werk betreft hadden wij weinig gemeen, maar verder konden we het goed vinden. Er was altijd tijd voor een praatje, of een discussie waarbij jij net als ik altijd je gelijk wilde halen; een goede eigenschap vind ik zelf ;-) Laat me weten wanneer de kinderboerderij klaar is, want ik heb Olivia beloofd dat we een keer naar Nicole en Dennis met hun eigen kinderboerderij gaan. De $<100$ heb ik misschien niet gehaald, maar we drinken toch een goede pint op het resultaat dat ik al geboekt heb, zowel voor wat betreft aantal pagina's als aantal verloren kilo's! Florence, jij begon als stagiair bij Caroline en mij. In het begin was je heel stil, maar later toen je eenmaal een tijdje als AIO bij ons werkte kwam je los. Je was nog steeds redelijk rustig, zeker in vergelijking met de rest, maar áls je wat zei dan was het ook meteen raak. Ruben, jij gekke Hollander! Je bent een vreemde vogel, maar je kunt heerlijk Chinees koken...... lekkerrrrr! An, zotteke, een gekke Belg! Tja, wat kan ik zeggen, een beetje onnozel natuurlijk, maar wel een toffe en plezante collega (ja, ik spreek inmiddels ook al een aardig woordje Vlaams).

Naast de AIO's zijn er nog andere mensen op de afdelingen Populatiegenetica en Klinische genetica die ik moet bedanken. Caroline, bedankt voor al het praktisch werk dat je voor mijn project verricht hebt. De resultaten van de arrays die voornamelijk door jou gerund zijn heb je nu in je handen! Erika, gek schaap jij met je schaap, samen met jou heb ik mijn eerste RNA isolaties gedaan. Ik kan me herinneren dat ik vond dat jij zo soepeltjes met die pipet omging. Bedankt voor de leuke en ontspannen praatjes, zomaar even op de gang of in de koffiekamer. Wanneer ga je nog eens met je moeder bellen zodat ik het Betuws dialect eens kan horen? Alexandra, mijn vraagbaak op $3 X$. Toen Rosy mij een rondleiding gaf, zei ze: "Dit is Alexandra, en als je hier iets nodig hebt dan moet je bij haar zijn”. Laat ik het zo zeggen, Rosy heeft niet gelogen! Bedankt voor je hulp! Torik, jou wil ik bedanken voor het feit dat je deur altijd open stond voor het stellen van een vraagje. Dat vraagje ontketende dan wel vaak een lange discussie, maar daar heb ik wel veel aan gehad. Bij deze wil ik me ook vast aanmelden voor de cruiseference op Curaçao of ergens anders waar het lekker warm is. Als ik als spreker kom, betaal jij vast mijn ticket en hotel, toch? Ton, bedankt voor je instant IT-hulp. Ook als je het erg druk had maakte je wel even tijd om mijn probleem op te lossen, zelfs op je wekelijkse vrije dag vanuit thuis. Succes met je nieuwe baan! Patrick, na een aantal jaren in Nederland gewerkt te hebben, neem ik aan dat je inmiddels je Nederlands cursus met succes hebt afgerond. Ik bedank jou dan ook in het Nederlands dat je $R$ aan mij geïntroduceerd hebt en een aantal analyses voor mij hebt gedaan. Je scripts waren niet altijd even eenvoudig, maar op den duur kon ik er toch goed wijs uit komen en zelfs wat zaken aanpassen. Rob, Ellen, Marion (of moet ik zeggen "Márion”), Jos D, Mike, Bieke, Marij en Fons, jullie natuurlijk ook bedankt voor mijn leuke tijd bij Popgen/Klingen. Rosy, jou moet ik natuurlijk niet vergeten te bedanken. Jij wist me altijd wegwijs te maken als er iets geregeld moest worden. 
Jij wist altijd waar en bij wie ik moest zijn. Ook waren de koffiekamersessies met jou altijd zeer vermakend.

Naast alle mensen van mijn oude werkplek, ben ik ook een "dikke merci" verschuldigd aan mijn nieuwe collega's in het verre Leuven (v.w.b. treintijd dan...). Ruth ("de ouw van de MAF"), Kizi (is het nu "Kiezzie”, “Kizzie” of “Kèzzie”?), Lieze (zeikwijf ;-) ), Joke (“dikke kak”), Kris (“is dah?") en Paul, jullie wil ik bedanken voor het feit dat jullie het aangedurfd hebben om een Hollander in jullie groep op te nemen. Ik heb het erg naar mijn zin bij jullie, en hoop dat ik nog een tijd mag blijven. Joke, bedankt voor je luisterend oor in de tijden dat ik het even nodig had. Paul, het "binnen afzienbare tijd" afronden van mijn doctoraat (zie mijn sollicitatiebrief) heeft langer geduurd dan verwacht, maar nu ben ik eindelijk ook officieel een volwaardige postdoc. Ik wil jou daarom in het bijzonder bedanken voor je geduld, je steun, en je vertrouwen in mij.

Mijn familie moet ik zeker bedanken! Guido, grote broer ;-), wij hadden het wel eens over de verschillen tussen jouw en mijn werk. Jij als consultant in de hoek van bank- en verzekeringszaken, en ik als wetenschappelijk onderzoeker in de genetica. We snapten weinig van elkaars werk, maar één ding was duidelijk: veel zaken zijn anders geregeld in deze sectoren. Bedankt voor onze spaarzame, maar toch ontspannen gesprekken hierover, die mij geholpen hebben bij het relativeren van sommige zaken. En dan nu het moeilijkste stuk...... Pap en Mam, jullie mag ik natuurlijk niet vergeten. Ik wil jullie met de woorden bedanken, precies zoals ze op dit moment in me opkomen. Pap en Mam, geer höb miech altied begeleid en gesteund in mien ganse studietrajec. Allewel geer neet altied begreep boe iech mèt bezig waor, vroog geer altied wie ut op 't wèrrek waor en of ' $t$ al get opsjoot. Zonder eure steun en raod, waor 't mesjiens gans aanders oetgepak. lech weit nog tot iech op 't punt stond um te stoppe mèt mien studie um mien favoriete biijbäönsje tot mien wèrrek te make. Allewel geer dat eigelik gein good idee vónt, stónt geer toch achter mien beslissing, moch iech de beslissing numme um te stoppe. Mer gelökkig höb geer miech toch weite te euvertuige um nog evekes door te zètte. 'T resultaat is d'r noe en iech bin kóntent, en iech hoop tot geer daor zjus zoe euver dink. Pap, ik sjrief dit stök vaan mien daankwoord mèt traone in mien ouge. lech weit totsdiech hiij hiel gere biij hads wèlle zien, en veer hadde aoch allemaol hiel gere gehad totste d'rbiij waors, mèr het heet neet zoe mage zien. lech mis diech daan oach hiel erg hiijbiij. lech hoop totsdiech 't toch allemaol u bitteke mètkrijgs en totsdiech tróts op miech bis. Pa en Ma, bedaank!

Ik moet natuurlijk niet vergeten mijn eigen gezinnetje, dat weer uitgebreid wordt met een tweede dochtertje, te bedanken. Olivia, je kunt dit nu nog niet lezen, maar misschien kijk je later nog eens in het dan stoffige boekje van Papa. Hoewel het zeker het afgelopen jaar heel zwaar is geweest, was jij altijd in staat om mij op te vrolijken na weer een lange dag werken. Stiekem hebben we je altijd wat langer op laten blijven, zodat ik je nog een uurtje kon zien als ik 's avonds thuiskwam van mijn werk in Leuven. Jouw lach, je lieve stemmetje en je knuffeltjes hebben mij erdoorheen gesleept! Ik hoop dat je zusje net zo lief en braaf wordt als jij, dan hebben mama en ik het héél erg 
goed. Claudia, jij hebt waarschijnlijk de meeste nadelen ondervonden van mijn promoveren. Vooral in de eindfase was er heel vaak geen tijd voor jou, zeker toen je de spaarzame vrije tijd ook nog moest delen met Olivia en andere zaken die noodgedwongen om mijn aandacht vroegen. Thuis van mijn werk, na het eten en nadat we Olivia in bed hadden gelegd, moest ik vaak nog "even snel" iets afmaken waar ik in de trein al aan begonnen was. Dat "even" werd vaak tot in de kleine uurtjes 's nachts. Toch heb jij zelden hierover geklaagd en ben je altijd mijn steunpilaar geweest en gebleven als er weer eens iets tegenzat. Ik kon altijd mijn verhaal bij jou kwijt, ook al begreep je soms niet waar het over ging en ook al klonk het soms allemaal wat onwerkelijk. Nu dit eindelijk is afgerond, kunnen we weer wat meer ontspannen van elkaar en van Olivia en haar zusje genieten. Claudia, jij bent de beste!

Ik heb ongetwijfeld mensen niet genoemd of ben mensen vergeten te noemen. Dat wil niet zeggen dat ik jullie niet dankbaar ben, maar het is gewoon onmogelijk om op dit moment aan iedereen te denken en dat op papier te zetten. Mensen, bedankt!

Rudy! 


\section{Curriculum Vitae}

Rudy Gerardus Elisabeth van Eijsden werd geboren op 3 augustus 1977 in Maastricht. Van 1989 tot 1995 doorliep hij het Voorbereidend Wetenschappelijk Onderwijs (VWO) aan het St. Maartenscollege te Maastricht waarna hij koos voor de opleiding Biologische Gezondheidkunde aan de Universiteit Maastricht. Na zijn afstudeerstage bij de afdeling Pathologie van het Academisch Ziekenhuis Maastricht met "een klonaliteitsanalyse van Non-Hodgkin lymfomen" als onderwerp, behaalde hij zijn universitair diploma in 2001. In 2001 werkte hij een korte tijd als wetenschappelijk onderzoeker bij de Universiteit Maastricht op de afdeling Gezondheidsrisico Analyse en Toxicologie en hielp daar bij het schrijven van een wetenschappelijk rapport over de toepassing van high-throughput genexpressieprofileringstechnieken in de exotoxicologie. Dit bleek later een goede voorbereiding geweest te zijn voor zijn promotieonderzoek waaraan hij in maart 2002 begon als assistent in opleiding (AIO) aan de Universiteit Maastricht bij de afdeling Klinische Genetica binnen de capaciteitsgroep Genetica en Celbiologie, verbonden aan het onderzoeksinstituut Groei en Ontwikkeling (GROW). Het onderzoek was gericht op oxidatieve fosforyleringsstoornissen en had als doel meer inzicht te verkrijgen in de moleculair biologische processen die bij deze stoornissen betrokken zijn. Het voornaamste middel waarvan binnen dit onderzoekstraject gebruik werd gemaakt was de microarray techniek, voor zowel genexpressiebepalingen als voor resequencing doeleinden. Vanaf 1 december 2007 is hij werkzaam als wetenschappelijk onderzoeker bij de MicroArray Faciliteit van het VIB te Leuven in België waar hij als postdoc meewerkt aan het YING (Yeast In No Gravity) project, waarbij de invloed van micrograviteit bestudeerd wordt op cellulaire adhesie, biofilmvorming en invasieve groei bij het modelorganisme Saccharomyces cerevisiae. Daarnaast is hij bij de VIB MicroArray Facility betrokken bij de planning van experimenten, het uitvoeren van data- en functionele analyses, en bij bioinformaticaprojecten in de meest brede zin van het woord. 
Abbreviations

\begin{tabular}{|c|c|}
\hline adPEO & autosomal dominant progressive external ophthalmoplegia \\
\hline AIC & Akaike information criterion \\
\hline BN-PAGE & blue native polyacrylamide gel electrophoresis \\
\hline CDF & probe set definition \\
\hline CDNA & complementary DNA \\
\hline $\operatorname{cox}$ & cytochrom c oxidase \\
\hline CPEO & chronic progressive external ophthalmoplegia \\
\hline DAVID & Database for Annotation, Visualisation and Integrated Discovery \\
\hline DCE & denaturant capillary electrophoresis \\
\hline DFP & diisopropyl fluorophosphate \\
\hline DGGE & denaturing gradient gel electrophoresis \\
\hline DHE & dihydroethidium \\
\hline dHPLC & denaturing high-performance liquid chromatography \\
\hline DMD & Duchene muscular dystrophy \\
\hline DTT & dithiothreitol \\
\hline EM & extracellular matrix \\
\hline ER & endoplasmatic reticulum \\
\hline GDAS & GeneChip DNA Analysis Software \\
\hline GENMAPP & Gene Map Annotator and Pathway Profiler \\
\hline HBSS & Hanks balanced salt solution \\
\hline KSS & Kearns-Sayre syndrome \\
\hline LHON & Leber hereditary optic atrophy \\
\hline MAC & membrane attack complex \\
\hline MELAS & mitochondrial myopathy, encephalopathy, lactic Acidosis, and stroke-like episodes \\
\hline MERRF & myoclonus epilepsy and ragged red Fibres \\
\hline MRI & magnetic resonance imaging \\
\hline mtDNA & mitochondrial DNA \\
\hline NARP & neuropathy, ataxia, and retinitis pigmentosa \\
\hline nDNA & nuclear DNA \\
\hline OXPHOS & oxidative phosphorylation \\
\hline PBS & phosphate-buffered saline \\
\hline PCR & polymerase chain reaction \\
\hline PDHC & pyruvate dehydrogenase complex \\
\hline PIM & protease inhibitor mixture \\
\hline QRT-PCR & quantitative real-time polymerase chain reaction \\
\hline RCRS & revised cambridge reference sequence \\
\hline ROS & reactive oxygen species \\
\hline RRF & ragged red fibres \\
\hline SD & standard deviation \\
\hline SSCP & single strand conformation polymorphism \\
\hline TGGE & temperature gradient gel electrophoresis \\
\hline UV & unclassified variant \\
\hline ww & wet weight \\
\hline
\end{tabular}


Stellingen behorende bij het proefschrift

\section{Microarray analysis of oxidative phosphorylation disorders}

Rudy Gerardus Elisabeth van Eijsden

Maastricht, 22 mei 2008 
1. Nieuwe krachtige sequencingtechnieken zullen eerder de oplossing vormen voor de genetische diagnostiek van complexe stoornissen dan slimme klinische classificaties. (dit proefschrift)

2. Stimulering van het OXPHOS systeem en de aanmaak van mitochondria stelt het optreden van klinische symptomen bij dragers van de m.3243A>C mutatie uit. (dit proefschrift)

3. De expressie van complement component $\mathrm{C}_{3}$ in spier is een indicatie voor de pathologie, ernst en progressie van OXPHOS aandoeningen. (dit proefschrift)

4. Inductie van weefselregeneratie middels complement component $\mathrm{C}_{3}$ is een centraal, maar ontoereikend herstelproces bij OXPHOS aandoeningen. (dit proefschrift)

5. Kleine genexpressieveranderingen krijgen pas betekenis wanneer ze gezamenlijk op groeps- of procesniveau bestudeerd worden.

6. Het sequencen van het complete genoom is een steeds nader komend feit in de genetische diagnostiek waarbij interpretatieproblemen als nevenverschijnsel geaccepteerd zullen moeten worden.

7. Hoe complexer de ziekte, hoe meer patiënten er nodig zijn voor een genexpressiestudie.

8. Dataopslag en -transport zal met de nieuwe generatie sequencingtechnologieën een groot probleem worden.

9. Het begrip resequencing suggereert een herhaling die er niet is.

10. Het gebruik van nieuwe krachtige sequencingapparaten zal aanzienlijk toenemen als bij elk apparaat een bioinformaticus geleverd wordt.

11. Het mtDNA is the circle of life. 